\title{
Scientific Exhibition
}

\section{CONTENTS}

PAGE

We will discuss WHEN and HOW to apply these treatments and make an evaluation of the results obtained and of any complications that may have arisen.

\section{SPACE 1295}

Bronchial atresia - report of 4 cases

A. Alvarez Castells (Presenter), J. Capellades, C. Torrents, P. Vicente de Vera, L. Gifre, M. Noguera; Barcelona

Bronchial atresia is a rare, well known congenital abnormality. There are reported less than 100 cases in the literature. It is discovered commonly as an incidental finding in young adults.

Chest radiology (Topic 01)

Cardiovascular radiology (Topic 02)

Mammography (Topic 03)

Gastrointestinal and abdominal radiology (Topic 04)

Uroradiology (Topic 05)

Musculo-Skeletal radiology (Topic 06)

Neuroradiology (Topic 07)

Head and neck radiology (Topic 08)

Pediatric radiology (Topic 09)

Contrast media (Topic 10)

Magnetic resonance (Topic 11)

PACS (Topic 12)

Education (Topic 14)

Technical developments (Topic 15)

Radiotherapy (Topic 16)

General radiology (Topic 17)

\section{SE}

\section{Chest radiology (01)}

\section{SPACE 1293}

Radiological patterns of chest involvement in Pneumocystis carinii Pneumonia

J. Albillos Merino (Presenter), J.Ferrando Alvarez-C., B. Gómez Ansón, R. Rubio Garcia, J. Manrique Chico; Madrid

Purpose: A number of patterns of chest involvement in AIDS-patients with PCP are shown. Differences between typical and atypical manifestations are presented. Several features in radiological evolution are noted.

Methods: Radiological evolution of 25 patients with PC.P was studied and plain PA and L films were reviewed. Films were taken at different moments during the disease: a) before admittance, b) at admittance, c) before treatment, d) during treatment, and e) after clinical improvement.

Results: Interstitial reticular or reticulo-nodular infiltrates are the most frequent pattern found in our study. There is a wide variety of other patterns, known as atypical, which, although less frequent, may appear during this disease. Several films of typical and atypical cases are shown, including atypical features like lobar consolidation, cavitation, apical infiltrates mimicking TBC, pneumatoceles, pleural effusion, a.s.o.

Conclusions: Recognizing the different patterns of chest involvement is of great importance in suggesting diagnosis of PCP, as well as in differentiating this entity from other infectious or non-infectious diseases in AIDS-patients.

\section{SPACE 1294}

The Interventional radiology of chest

X. Alegret (Presenter), J. Palmer, J. Mata, J. Caceres M. Sentis, Y. Pallardo; Barcelona

The Interventional Radiology of chest has acquired an important role in the diagnosis and treatment of many thoracic illnesses. The fluoroscopy, ultrasounds and C.T. give a clear enough picture of the lesions of the thoracic wall, pleura, lungs and mediastinum. To permit the accurate percutaneous access to these lesions.

Among the interventional procedures we have carried out in our department are the fine-needle aspiration biopsy of chest and the treatment of the pleural and mediastinal collections by drainage using very narrow catheter. We have used these techniques to treat empyema, pneumothorax, abscesses, mediastinal cysts and pericardial collections.
In the past, the diagnosis was made surgically. Nowadays the condition is characterized by nearly pathognomonic radiologic features wich have significant implications in the patient management.

The plain film and CT findings consists in a lobar hyperinsuflation associated with bronchomucocele. Endoscopic examination is necessary for excluding other causes of mucoid impactation. If the diagnosis is supported by both radiological and endoscopic methods, conservative treatment is recommended.

We present 4 cases of bronchial atresia in young adults and discuss the CT, DAS, bronchographic and plain film findings. We emphasize the necessity of to be used with this condition in order to prevent unnecessary further explorations, including thoracotomy.

\section{SPACE 1296}

MacLeod's syndrome: radiological findings

A. Alvarez Castells (Presenter), E. Pallisa, C. Torrents, S. Coll, L. Gifre, P. Vicente de Vera; Barcelona

MacLeod (or Swyer-James) syndrome is often diagnosed with plain chest radiographs. Other radiological procedures are also very useful to confirm the suspected diagnosis.

Features of distinct radiological procedures such as scintigraphy of the lung, angiography, bronchography and C.T in 7 patients with MacLeod syndrome are presented and evaluated comparatively.

Plain chest radiography shows a hyperlucent lung of reduced or normal size with diminished vascular markings and little change in lung volume during respiration.

Pulmonary scintigraphy shows markedly decreased perfusion in the affected lung. The perfusion image alone does not exclude other vascular abnormalities. Ventilation-perfusion imaging is helpful in confirming the presence of this syndrome.

C.T findings are:

1. reduction of attenuation values of the affected lung

2. loss of normal anteroposterior gradient attenuation

3. diminishing of central and peripheric pulmonary arteries.

Other associated findings, in C.T, include parenchymal changes such as bronchiectasis.

Conventional or digital pulmonary angiography demonstrates the ipsilateral pulmonary artery to be smaller than normal with thin branches and diminished vascularity in capillary and venous phases.

\section{SPACE 1297}

Abnormalities of lung development: a continuum spectrum of abnormal vascular and parenchymal development

S. Borruel (Presenter), C. Lopez, J. L. Raya, J. Albillos, J. Roldan; Madrid

The major abnormalities of lung development can be classified into six discrete categories: pulmonary arteriovenous malformation, hypogenetic lung syndrome, bronchopulmonary sequestration, congenital cystic adenomatoid malformation, bronchogenic cyst and congenital lobar emphysema. These major abnormalities should be considered as a continuum spectrum of lung malformation, with pure vascular abnormalities on the one hand (i.e. pulmonary arteriovenous fistula) and pure parenchymal malformations on the other (i.e. congenital lobar emphysema). Between these "pure" abnormalities there are other major abnormalities with different degrees of vascular and parenchymal compromise, and some minor or overlap syndromes that present features of more than one major anomaly.

The aim of this presentation is to show cases of each of these major anomalies of lung development, studied by conventional radiology, CT scanning and vascular procedures. We also make a differential diagnosis of these conditions, highlighting the typical features of each one. 


\section{SPACE 1298}

The value of transthoracic percutaneous biopsy of focal pulmonary lesions

P. Bracke (Presenter), B. Corthouts, A. De Schepper; Edegem

In a retrospective study, 218 fine needle aspiration lung biopsies were performed in 200 patients with an age distribution from 4 to 88 years. $85 \%$ of the punctions were performed to exclude malignancy.

By using cutting biopsy needles, the amount and quality of biopsied tissue (tissue core instead of cytologic material) allowed histopathologic evaluation in $89 \%$. In 125 patients malignancy was confirmed and further histopathologic differentiation was possible in $54 \%$.

The rate of intraparenchymatous hemorrhage and pneumothorax were concordant with literature findings.

Transthoracic lung biopsy is a major diagnostic tool in the evaluation of:

1. lung lesions of unknown etiology that are not likely to require resection

2. metastatic disease

3. two lesions in different lung lobes

4. opportunistic infections in patients with immunosuppression

\section{SPACE 1299}

\section{The dense azygos lobe: a normal variant}

J. Cáceres (Presenter), J. M. Mata, X. Alegret, J. Palmer; Barcelona

Purpose: To demonstrate that a dense azygos lobe that looks abnormal on the PA chest radiograph may be a normal variant.

Methods and Materials: Eight patients with a dense azygos lobe on PA chest radiographs were studied with $\mathrm{CT}$. Slices were $8 \mathrm{~mm}$ thick, with a $10 \mathrm{~mm}$ interval.

Results: None of the patients had any pathologic process of the lung or mediastinum. All of them had a shallow azygos lobe and increased depth of the mediastinal soft tissues in front of it. We measured the depth of the mediastinal soft tissues and of the lung within the azygos lobe at the level of the sternoclavicular joint. The ratio soft tissue/lung was greater than one in all our patients, whereas it was less than one in ten other patients with normalappearing azygos lobes. The cause of the increased depth of the mediastinum was marked elongation of the great vessels in seven cases and tumor infiltration of the right chest wall in one.

Conclusions: We conclude that the increased density is due to the combination of a shallow azygos lobe and increased depth of the mediastinum, caused in the majority of cases by elongated vessels.

\section{SPACE 1300}

Atypical features of free pleural effusion by $\mathrm{CT}$

J. Cáceres (Presenter), J.M. Mata, J.Palmer, L. Donoso, E. Castañer; Barcelona

Characteristically, free pleural fluid has a lenticular shape and uniform density on CT studies.

In the last two years we have seen several cases of pleural effusion that show atypical features, either in shape or in density. In some cases, free pleural fluid adopts a convex shape simulating encapsulation. In other cases, the fluid had a nodular appearance, mimicking pleural masses. In one case, fluid in an azygos lobe simulated a mediastinal mass. In all cases, the diagnosis was easily made with prone C.T, that showed that the fluid moved freely within the pleural space.

Increased density of the fluid may be secondary to bleeding. Localized dense areas suggest metastatic implants or primary tumors. Occasionally a dark transverse band within the fluid is seen, representing displaced extrapleural fat.

\section{SPACE 1301}

High-resolution CT of interstitial lung disease: differential diagnosis E. Castella Fierro (Presenter), A. Alvarez Castells, C. Torrents Odin, L. Gifre Bassols, P. Vicente de Vera, M.D. Miranda; Barcelona

High-resolution CT appears to be more sensitive than CT and conventional radiographs for the detection of lung abnormalities.

CT has become capable of imaging the lung with excellent spatial resolution and anatomic detail, demonstrating both the normal and abnormal interstitium, as well as morphologic characteristics of localized and diffuse parenchymal processes.

Alteration in anatomy can be identified at the level of the secondary pulmonary lobule, and although often nonspecific, in certain situations high-resolution $\mathrm{CT}$ findings can be diagnostic.

In basis to the HRCT findings, we classified the clinical entities in the following groups:

- distortion of the secondary pulmonary lobule (scleroderma)

- bronchovascular bundle thickening (lymphangitic carcinomatosis).
- presence of cysts (sclerosis tuberose, histiocytosis X, lymphangioleiomyomatosis, emphysema).

- nodules (silicosis, sarcoidosis):

- associated findings (subpleural line, patchy air-space disease).

\section{SPACE 1302}

Traumatic hernias of the diaphragm

I. Castells Ferrer (Presenter), R. Dominguez Orozco, E. Castellà, J. Capellades, J. Alvarez, C. Triginer; Barcelona

The traumatic lesions of the diaphragm are not rare condition, but usually the diagnosis is delayed due to the absence of specific clinic and radiologic signs. As a consequence of diaphragmatic tears is frequent the herniation of abdominal viscera or omentum.

We present eight cases of traumatic hernia of the diaphragm, surgically comproved. Seven were caused by blunt trauma and one by penetrating injury. We discuss the radiologic findings on portable plain film that may suggest the possibility of diaphragmatic rupture.

\section{SPACE 1303}

High resolution computed tomography in diseased isolated lungs: a radiologic-pathologic correlation

S. Diederich (Presenter), N. Roos, H. Lenzen, Z. Puskas, A. Fahrenkamp. U. Bick, P.E. Peters; Münster

Purpose: The aim of our study was to establish recise correlations between high-resolution computed tomographic (HRCT) scans and corresponding gross pathological sections.

Methods and Materials: 22 isolated lungs were fixed with a solution containing polyethylene glycol 400 and dried by air inflation applying a constant pressure of $25 \mathrm{~mm} \mathrm{Hg}$. HRCT scans were obtained in standard positions and gross pathological sections were prepared at exactly identical levels. A matrix was projected to CT scans and pathological sections to allow precise correlation between CT, macro- and microscopic findings.

Results: The method applied, particularly with the matrix technique, allowed an exact regional HRCT-pathologic correlation. The patients studied included: $A R D S, n=4$, pneumonia, $n=3$, tuberculosis, $n=2$, multiple lung abscesses, $n=1$, COPD, $n=1$, pulmonary embolic infarction, $n=1$, intoxication, $n=1$, Goodpasture's syndrome, $n=1$, pulmonary metastasis, $n=4$, pulmonary lymphoma, $n=2$. We observed, that even with well established CT-phenomena complex differential diagnoses often had to be considered. Conclusions: Postmortem pathologic correlation of pulmonary HRCT applying the matrix technique allows precise characterization of radiologic findings. We therefore conclude that through these observations an optimization of in vivo pulmonary CT imaging can be obtained.

\section{SPACE 1304}

\section{Adrenal and pancreatic metastases of lung cancer}

M. Elwertowski (Presenter), M. Andrzejewska, J. Gryminski, M. Stepniewski, A. Drygalska-Pozoronska, L. Pawlicka; Zalesie Gorne

Abdominal ultrasonography showed to be extremely useful in diagnosis of the spread of lung cancer. We tried to evaluate its utility in diagnosis of seldom described metastases to adrenal glands and pancreas.

Material of the work consists of 800 patients with histologically recognised lung cancer in whom 2150 ultrasonic examinations were performed. Intraabdominal metastases were found in 147 cases $(18,4 \%)$ being encountered most frequently in the liver (84), adrenals (66) and abdominal lymph nodes. CT was performed to confirm US study results.

The size of demonstrated adrenal tumors varied form 7-10 $\mathrm{mm}$ when located in the right gland and $10-14 \mathrm{~mm}$ in the left gland to several centimeters. Metastases were found in $\mathbf{3 7}$ out of $\mathbf{1 7 5}$ patients with small cell carcinoma $(21 \%), 14 / 143$ cases of adenocarcinoma $(10 \%), 7 / 305$ cases of carcinoma planoepitheliale $(2,4 \%)$ and $8 / 177$ of other lung neoplasms. Out of 12 patients with metastases to pancreas 10 were affected with small cell carcinoma. Ultrasonic images of all smaller changes (under $2 \mathrm{~cm}$ diameter) presented hypoechoic, homogeneous well defined foci localized within pancreatic tissue, bigger metastases appeared as hypoechoic lesions with uneven echogenicity that were not affecting pancreatic duct diameter.

CT proved to be superior in diagnosis of adrenal metastases (specially when localized on the left side) while US was better in pancreatic changes (CT failed to visualize 4 out of 12 tumors). Follow up studies of patients with small cell ca and adrenal metastases showed to be an useful indicator of chemotherapy effects. Enlargement of adrenals occurring BEFORE lung progression suggests earlier chemotheraphy. 


\section{SPACE 1305}

\section{Thoracic MR after pneumonectomy}

C. Frola (Presenter), S. Cantoni, J. Serrano, C. Leoni; Genova

Purpose: Radiologic follow-up of patients who undergo pneumonectomy for bronchogenic carcinoma can be divided in two periods: the immediate postoperative control, from surgery to discharge, and further follow-up. While postoperative management is a nearly exclusive radiologic care, TC and MR are essential for further follow-up. We undertook this study to evaluate the utility of MR in this field, presuming that peculiar anatomic feature of the operated emithorax with the postpneumonectomy space full of fluid or organized tissue, in the absence of respiratory movements is an ideal condition for MR imaging.

Methods: From 1986 to nowaday we examined 45 patients with a resistive whole body magnet operating at $0,15 \mathrm{~T}$ in the first two years, and with a superconductive whole body magnet operating at $0,5 \mathrm{~T}$ in the following years. All patients had symptoms and there was a strong suspicion of recurrence or other complications.

Conclusions: MR appeared extremely reliable in the evaluation of pneumonectomy space itself. By means of the different signal MR could differentiate fluid or organized tissue from recurrence.

\section{SPACE 1306}

Thickening of the posterior pleural junction line: a normal variant? P. Guembe (Presenter), L. Hernandez, I. Gordillo, T. Alvarez, J. Alarcon; Madrid

Purpose: The anatomic contact between the posterior portion of both lung apices, in the supra-azygos recess, is radiographically seen as a sharp, stripped, "y" shaped opacity projected upon the tracheal lumen and above the aortic arch. It's admitted that the maximum width of this line should be lesser than $3 \mathrm{~mm}$ representing the simple apposition of the four pleural layers separated by a small amount of mediastinal soft tissue. In our experience, it is relatively frequent to detect thickening of this posterior superior pleural junction line in asymptomatic patients without previous thoracic pathology.

Methods and Materials: We have thoroughly studied some of this patients with Computed Tomography finding that, in the vast majority of them, this thickening was explained by the interposition of the esophagus between the apical regions of both lungs. Occasionally, a mediastinal anomalous vessel was seen.

Results: We conclude that the posterior pleural junction line thickening on a posteroanterior chest film, as an isolated feature, is not of diagnostic value, being many times explained by the esophagus interposition, and so it may be considered a normal roentgen variant.

\section{SPACE 1307}

Ultrasonically guided fine needle aspiration biopsy of peripheral pulmonary masses

Abdullah Bostan, Fahrettin Hasircioğlu (Presenter), Nevin Tamac, Ahmet C. Kaur, İnci Köseli; Ankara

Advances in imaging technology, improvements in cytopathology and the use of fine needles extended the application of percutaneous transthoracic needle biopsies by making it possible to safely perform biopsy of intrathoracic masses. Application of ultrasound to the guidance of aspiration biopsy of the peripheral pulmonary lesions currently become the method of first choice because of its simplicity and low rate of complications.

We report our experience in ultrasonically guided aspiration biopsy in 40 patients who had peripheral pulmonary masses. The mass was analyzed with respect to the size, margin and internal sonographic pattern. Distances from the skin to the anterior and posterior surfaces of the mass were measured. We used a 22 gauge Chiba needle for the aspiration biopsy.

Ultrasonically guided percutaneous needle biopsy of pulmonary masses in contact with the chest wall is a safe simple procedure. The lack of ionising radiation, the relatively low cost and wide availability, the ability to visualise the anterior and posterior wall of the mass and the needle tip, which provides continuous guidance during the biopsy are advantages of ultrasound when compared with fluoroscopy and computerised tomography guided biopsy.

\section{SPACE 1308}

Positron emission tomography of the thorax - a new imaging technique Michael V. Knopp (Presenter), Ludwig G. Strauss, Uwe Haberkorn, Antonia Dimitrakopoulou, Helge Bischoff, Detlef Branscheid, Josef Doll, Hermann Ostertag, Gerhard van Kaick; Heidelberg

Purpose: The introduction of whole-body positron emission tomography enables metabolic oriented imaging of thoracic lesions. This exhibit presents selected PET studies of the thorax in conjunction with a CT, MRI and pathologic findings.
Methods and Materials: PET studies were obtained using a whole-body PET scanner with F-18-labelled deoxyglucose (FDG). Dynamic studies as well as endpoint measurements 15 post injection of $440 \mathrm{Mbq}$ FDG iv. were obtained. Emission scans of 15 minutes and transmission scans of 5 minutes were obtained 50 minutes post injection. Tracer uptake concentrations are quantified using the standardised uptake value. More than 150 PET studies of thoracic lesions have been performed.

Results: All malignant untreated tumors showed significant FDG uptake with uptake values above 1.8 SUV. All benign lesions showed significant lower FDG accumulation with some overlap for patients with tuberculosis. PET shows a great potential in the preoperative evaluation of indetermined lesions with a high sensitivity and specificity for $\mathrm{T}$ - as well as $\mathrm{N}$-staging. PET also shows tremendous potential in the evaluation of therapy response by correctly predicting the therapy success prior to midpoint of the protocol.

\section{SPACE 1309}

CT-pathologic correlative study of interstitial pneumonia Hiroyuki Koba (Presenter), Seiya Katoh, Hideaki Watanabe, Masahiko Yamagishi, Yuji Mori, Akira Suzuki; Sapporo

A direct CT-pathologic correlative study of interstitial pneumonia was performed using inflated and fixed lungs. The specimens, which were obtained from 26 patients at the time of autopsy or during surgery, had pathologic findings of interstitial pneumonia (UIP and/or DAD). The specimens were fixed in distension and air dried. They were scanned with GE CT/T9800.

HRCT images of the specimens could be correlated with two different types of pathologic processes. One was the chronic fibrotic change which was seen in the subpleural area and the other was the acute alveolar change which was seen in the diffuse area. HRCT images of the chronic fibrotic change showed nodular opacities, ring like opacities, increased density (high density), and air-bronchiologram. These findings were correlated with patchy fibrotic lesions, honeycombing, mucus stasis in cysts of the honeycomb and dilatation of the airways. HRCT images of the acute alveolar change showed mainly diffuse increased densities and diffuse nodular opacities. These findings were correlated with a diffuse thickening of the alveolar walls, hyaline membranes and their organization.

Understanding of the difference between HRCT images of these chronic and acute pathologic processes is helpful for the evaluation of disease processes of interstitial pneumonia by HRCT.

\section{SPACE 1310}

\section{Computed tomography of pleural fissures and a new scanning method} for detecting fissures

Y. Kodama (Presenter), S. Sakuma; Aichi-ken

A review was made of computed tomography of the pleural fissures by conventional and thin scan of the axial image, and a new technique of thin scanning is performed by tilting the gantry cephaladly to improve detection of the pulmonary fissures is discussed. All 203 cases were scanned sequentially from lung apex to base in inspiratory apnea, using contiguous $10 \mathrm{~mm}$ sections. Sections were partially obtained with collimation of $5 \mathrm{~mm}$ in 13 cases and $2 \mathrm{~mm}$ in twelve. Scanning was performed by tilting the gantry cephaladly to about 20 degrees with $5 \mathrm{~mm}$ collimation in 2 cases and $2 \mathrm{~mm}$ in 5 cases. Studies were performed with a TCT $60 \mathrm{~A}$ or $70 \mathrm{~A}$ (TOSHIBA; TOKYO). The present ce: $:=$ in which pleural fissures were recognized not as the hypovascular zone but as the opaque density are discussed. By conventional scanning with $10 \mathrm{~mm}$ collimation, a bilateral major fissure was recognized in 36 cases and unilateral major fissure in $\mathbf{4 3}$ cases. Sixty-six cases showed linear or bandlike density of the major fissure. In 38 cases, the major fissure was demonstrated by the difference in pulmonary density between the upper and lower lobes. By thin scanning with $5 \mathrm{~mm}$ and $2 \mathrm{~mm}$ collimation, all cases showed linear density of the major fissure. It was quite difficult to detect minor fissure as opaque densitiy in conventional scanning, but 5 of 13 cases with $5 \mathrm{~mm}$ collimation and 10 of 12 cases with $2 \mathrm{~mm}$ collimation showed an opaque line. In 7 cases of thin scanning by tilting the gantry cephaladly, pleural fissures could be seen more clearly than in other cases of axial thin scanning. Thin scanning is thus shown useful of detecting pleural fissures and improving detectability not only of the major fissure but of the minor fissure as well by thin scanning tilting the gantry cephaladly within the possible range.

\section{SPACE 1311}

Optimized procedure of description and interpretation of results for the assessment of chest X-rays

H.M. Kulke (Presenter); Würzburg

In order to improve and standardize the previously diverse and troublesome descriptions of radiological analysis, as well as to make use of computer analysis, the findings, which might be observed on a thoracic $X$-ray, were analyzed.

The chest X-rays of 2,684 inpatients and ambulatory patients with diseases of 
the lung and bronchial system as well as the cardiovascular system were examined. Exact regions of description were defined in which findings of description, the detailed characteristics of pathological changes and phenomena, including foreign objects and anomalies, might be observed. Using this basis, and taking in consideration the features of individual diseases, both the radiological examination of the thorax as well as its interpretation could be optimized and the findings were able to be stored in a computer.

\section{SPACE 1312}

\section{High resolution $\mathrm{CT}$ of the lung in scleroderma}

M. D. Miranda (Presenter), A. Alvarez Castells, C. Torrents, P. Vicente de Vera, L. L. Gifre, V. Fonollosa, C. P. Simeon; Barcelona

We prospectively studied 22 patients with know Progressive Systemic Sclerosis by using HRCT. The age of the patients varied from 42 to 68 years old, with a male to female ratio of $1: 4$. We correlated HRCT findings with chest radiographs and lung function tests. CT study consists of a scanning with contiguous $10 \mathrm{~mm}$ slice thickness from the apex to the diaphragm. Further thin sections $(1.2 \mathrm{~mm}$ ) were obtained at predetermined levels (aortic arch, carina and $1-3 \mathrm{~cm}$ above diaphragm). Additional scans were performed with the patient in the prone position.

The most characteristic pathologic findings were a diffuse reticular pattern with a predominantly basilar distribution, without nodules. There was evidence of distortion of secondary lobules eithout thickening of the intralobular interstitium. Thickening of interlobar fissure were frequently identified and subpleural line as well, typically described in Asbestosis and Pulmonary Fibrosis. Peribronchial thickening was seen not associated with bronchiectasis, cystic dilatations or emphysema. Another findings were recognized such as esophageal involvement.

Our data suggest that HRCT is more accurate than chest radiography and in some cases results more sensitive than lung function tests.

\section{SPACE 1313}

Radiologic-pathologic correlation of chronic eosinophilic pneumonia Yumiko Nakai (Presenter), Yoshirou Mochizuki, Tetsuji Kawamura, Rieko Kawanami, Yoichiro Kohashi, Toshiharu Nakai, Fumiyuki Kuze, Harumi Ito; Otsu

Purpose: Chronic eosinophilic pneumonia (CEP) and BOOP have some common radiologic, clinical and pathologic features. This study were undertaken to establish radiographic and C.T features of CEP based on pathologic study.

Methods and Materials: Open lung biopsy with informed concent was performed on 6 cases clinically diagnosed eosinophilic pneumonia which could not be confirmed by TBLB or which were difficult to distinguish from BOOP.

Results: All the cases had eosinophilia in peripheral blood (9-75\%) and BAL fluid (3-43\%). Chest radiographs and CT showed multiple alveolar shadows in the peripheral lung field (4 cases), both alveolar and interstitial shadows (1 case) and only interstitial shadow ( 1 case). Pathologically these radiologic abnormalities corresponded to infiltrate of eosinophils and other cell in the aveolar space and interstitial tissue of the lung (alveolar wall, inter lobular septum, peribronchiolar and vascular space and pleura), respectively. In 2 cases, typical organizing materials were found in both alveolar space and bronchiolar lumen, which was also responsible for airspace shadows.

Conclusions: The most important pathologic features of CEP were a wide aspect of interstitial involvement of the lung in addition to airspace consolidation, while in BOOP interstitial involvement was confined to the alveolar septum.

\section{SPACE 1314}

Follow-up of emphysema associated with alpha 1 antitrypsin deficiency by computed tomography

J. Neuwirth (Presenter), R. Finke, E. Dinkel; Praha

Purpose: We have evaluated computer tomography (CT) examinations of 9 patients, with alpha $I$ antitrypsin (AAT) deficiency before and after one year augmentation therapy to detect the progression of emphysema.

Methods: We have used simple visual grading score $(G)$ and also CT quantitative values to determine the extent of emphysema. The measured values were mean lung density (D), emphysematous area (EA) - the percentage of pixels with density below $-910 \mathrm{HU}$. We correlated this values to each other and compared the average values before and after therapy.

Results: We have confirmed significant correlation between $E A$ and $G$ $(r=0.841), G$ and $D(r=-0.731)$, interobserver-G and $G, E A$ and $D$ $(\mathrm{rz}=-0.884)$. Spearmann rank correlation coefficient with $\mathrm{p}<0.001$ was used. The average progression of the density was only $2 \mathrm{HU}(-87)$ resp. -873 HU), more distinct was progression of EA - $6 \%(40 \%$ resp. $46 \%)$ and grading 0.17 (2.48 resp. 2.65). We have confirmed higher progression of emphyse- matous changes at the basal region. The evolution of $\mathrm{CT}$ values was very similar to the evolution of pulmonary function parameters.

Conclusions: The CT is a sensitive method for the follow-up of emphysematous caused by AAT deficiency. The EA value is more sensitive than D because of development of bullae with dense wall by some patients. We propose an algorithm for the follow up with $\mathrm{CT}$, emphasizing the advantage of the routine quantitation of the extent of emphysema.

\section{SPACE 1315}

The effectiveness of thin-section CT for lung field nodular shadows Harutaka Niiya (Presenter), Nobuhiro Inoue, Toshiaki Shirakami, Shin Kimoto, Nobuo Sato, Yoshio Hiraki; Okayama

Purpose: Thin-section CT was performed for lung field nodular shadows and a study was made of its effectiveness.

Subject and methods: Subjects consisted of 22 cases of histologically diagnosable lung field nodular shadows. The breakdown of those subjects consists of 13 benign cases and 9 malignant cases.

Thin-section CT using $2 \mathrm{~mm}$ slices was performed while interrupting breathing using the Toshiba Model TCT-900S. Studies were then made of the properties of the borders of the nodular shadows (notches, spiculas, etc.), pleural indentation, distal convergence of peripheral blood vessels, node internal structure and density non-uniformity and so on.

Results: Diagnosis of benignity or malignancy was made with $89 \%$ sensitivity, $77 \%$ specificity and $82 \%$ diagnostic accuracy. A detailed study of the distal convergence of peripheral blood vessels revealed that this can be a major factor for improving diagnostic accuracy.

\section{SPACE 1316}

The value of chest CT in the screening for aortic tear in patients with blunt trauma

Vassilios Raptopoulos (Presenter), Robert G., Sheiman, David A. Phillips, Ashley Davidoff, Wayne Silva; Boston, MA

Purpose: The objective of this study was to examine the role of chest CT in the screening for thoracic aortic injury.

Methods: Dynamic chest CT was done in 326 patients who had an abdominal CT for blunt trauma over a two-year period. During the same time we encountered 142 additional patients with chest trauma requiring aortography. Results: Of 468 patients, 244 (52\%) had abnormal chest radiography, of whom only $18(7 \%)$ had abnormal aortography. Three patients with normal radiography had aortic tear; two had chest CT which was abnormal in both. In comparison, chest CT was abnormal in only $54(17 \%)$ of 326 patients, and of these $10(19 \%)$, had abnormal aortography. There were no false negative scans, while $79 \%$ of patients with normal CT had false positive chest radiography. Compared to chest radiography, there was no significant difference in sensitivity ( $86 \%$ vs $100 \%)$, but with CT there was a significant improvement in specificity $(49 \%$ vs $86 \%)$ and accuracy. Had chest CT been used as a screening method, the need for aortography would have decreased by $67 \%$ while the rate of positive aortograms would have increased by $63 \%$.

Conclusions: Chest CT can safely discriminate candidates for aortography, it is cost effective and, in hemodynamically stable patients, it should be incorporated in the screening for traumatic aortic tear.

\section{SPACE 1317}

DSA as the method of choice for pulmonary and bronchial angiography C. Richter (Presenter), G. Schröder, K. Richter; Berlin

A method of procedure for pulmonary and bronchial DSA is described, in which morphological information of high detail recognition, on the one hand, and functional information on pulmonary perfusion on the other hand, can be obtained in parellel during one and the same examination. The results obtained from more than 400 cases with various pulmonary and cardiovascular diseases disprove the still widespread view that conventional angiography continues to be the "gold standard" even in the age of digital methods. The poster demonstrates in detail the possibilities and conditions of technique, handling, image processing, post processing with time density curves and the joint interpretation of images and curves.

\section{SPACE 1318}

Evaluation of magnetic resonance imaging in thoracic inlet tumors Eiro Sakai (Presenter), Shuji Adachi, Koji Tanaka, Eiichiro Itouji, Masahiko Kusumoto, Michio Kono; Kobe

Purpose: MRI Evaluation of the detectability of tumor invasion to the thoracic inlet.

Methods and Materials: MRI was performed in 33 lung cancer patients and the diagnostic accuracy of MRI was compared with that of CT concerning the utility for thoracic inlet lesions. We assessed abnormal findings in comparison 
with surgical or autopsy findings.

Results: In the local extent of the tumor, the accuracy for tumor invasion to the vessels such as subclavian artery and vein was $95 \%$ for MRI, and $88 \%$ for CT. MRI was slightly superior to $C T$, but MRI was equivalent to $C T$ with regard to invasion to the base of the neck, lateral chest wall, ribs, and vertebral bodies. However on MRI, it is easier to understand the longitudinal tumor extent than on CT. CT has superior spatial resolution but CT has also disadvantages, such as streak artifact caused by shoulder joints, resulting image degradation. In contrast, MRI has inherent advantages, and multiple images which facilitate the relationship between tumor and normal structures. Coronal and sagittal MR images facilitated 3-D observation of tumor of invasion in the thoracic inlet.

Conclusions: MRI was useful in deciding surgical indication and the planning for radiotherapy.

\section{SPACE 1319}

\section{A computerized system to classify chest radiograms by existing} nodules

Hirotsugu Takabatake (Presenter), Masaki Mori, Syoichi Sasaoka, Hiroshi Natori, Hideo Suzuki, Noriko Inaoka, Akira Suzuki; Sapporo

Purpose: The cure of pulmonary adenocarcinoma greatly depends upon the detection at an early stage. It is known that solitary pulmonary nodules on chest radiograms failed to detect as many as $30 \%$ of the positive cases. In order to increase the accuracy of detecting pulmonary nodules, a computerized system to classify chest radiograms by existing nodules was developed.

Methods and Materials: This system used digital image-processing techniques based on the information which were extracted from 266 chest radiograms (192 with a cancerous nodule and 74 normals). This system can a) detect nodules using a previously developed called as Directional Contrast Filter for Nodules which is effective for detecting nodules with obscure margins, b) eliminate false positive foci and c) classify radiograms by existing nodules. An experiment using 108 practical radiograms (30 with nodules and 78 normals) was performed. Each nodule on a chest radiogram is pathologically proven to be primary pulmonary carcinomas and less than $30 \mathrm{~mm}$ in diameter.

Results: As a result, figures of $76.7 \%$ for sensitivity and $69.2 \%$ for for specificity of the system were obtained. This result indicates that the system will be useful to further increase the accuracy of detecting pulmonary nodules.

\section{SPACE 1320}

Mycoplasma pneumonia: CT assessment and correlation with chest radlographic findings

Hiroshi Tanaka (Presenter), Hiroyuki Koba, Takuji Mori, Mitsuo Asakawa, Akira Suzuki; Sapporo

Purpose: To identify and characterize the CT manifestations of mycoplasma pneumonia (MP) and to evaluate the contribution of CT compared with chest radiography in estimating disease severity.

Methods and Materials: A total of 65 pairs of CT scans and chest radiographs obtained in patients with serologically proved MP, clinically mild cases (group $1, n=55$ ) and severe cases with hypoxemia (group $2, n=10$ ), were analyzed retrospectively.

Results: Three characteristic CT patterns of MP were demonstrated. First, thickness of the bronchoarterial bundles: $92 \%$ (group 1), 90\% (group 2). Second, nodular opacities which having a centrilobular distribution, at the ends of the branches of the pulmonary arteries and bronchi: $92 \%$ (group 1), $100 \%$ (group 2). Third, consolidation with an air bronchogram : $72 \%$ (group 1), $90 \%$ (group 2). Mediastinal or hilar lymph nodes swelling was noted $20 \%$ (group 1), 70\% (group 2), pleural effusions were revealed 17\% (group 1), $70 \%$ (group 2). In 10/65 patients, chest radiograph could not detect the thickness of bronchoarterial bundles nor nodural shadows that CT could. The superiority of CT over chest radiography was supported in the early detection of small opacities, and recognition of mediastinal or hilar lymph nodes swelling and small amount of pleural effusions that important in estimating MP severity.

\section{SPACE 1321}

Evaluation of small airway lesions using computed tomography, selective alveolobronchography and ultrathin bronchofiberscopes Mitsuru Tanaka (Presenter); Tokyo

Small airways less than $2 \mathrm{~mm}$ in diameter are injured by various causes and diseases. Among them, smoking and air pollution have now become social problems. A pathophysiologic understanding of these sites will give important clues to clarify the etiology of many pulmonary diseases. These pulmonary sites are called "silent zones" because they do not attract too much attention until the disease advances. We developed the first ultrathin endoscopes which facilitate observation and photography of small airways of less than $2 \mathrm{~mm}$ in diameter. We examined various pulmonary diseases by endoscopy, and obtained findings that are impossible to obtain by conventional roentgenography and endoscopy. In normal subjects and patients with pulmonary diseases, CT scan findings and selective alveolobronchograms were examined by conforming the sites of lung biopsy findings to those of endoscopic observation. As a result, we obtained findings of the small airways which have not been described in other publications.

\section{SPACE 1322}

Diagnosis of mediastinal vascular involvement in lung central cancer by methods of imaging

M. Torres (Presenter), P.Valdes, R.Varela, J.Vicente, F. Serrano, F. Rodriguez; Malaga

Purpose: The aim of this work is to show our experience in the value of different radiological diagnostic methods in the study of vascular invasion (pulmonary and aort arteries) in central lung cancer ( $\mathrm{T}-3$ versus $\mathrm{T}-4$ stage).

Methods: We present 8 male patients aged between 40 and 60 years with bronchoscopic diagnosis of centra bronchial epidermoid carcinoma. Posteroanterior and lateral thoracic radiography, CT with and without intravenous contrast, dynamic CT and Nuclear Magnetic Resonance were all performed in each patient. Arteriography was carried out in five. We compared our data with the surgical findings. We considered as criteria of invasion a contact angle greater than $90^{\circ}$, absence of fat-plane between tumour and vessel wall, and either concentric or non-concentric irregularity of the vessel lumen.

Results: The most useful criteria of invasion was the irregularity of the lumen in both pulmonary and aortic invasion, being present in $100 \%$ of the surgical positive cases. Of the imaging techniques, NMR in axial slices for the pulmonary artery and axial and coronal slices for the aorta, were the most useful in the diagnosis of the irregularity and its extension, surpassing the arteriography in cases where invasion is excentric or there is adventicial and perivascular affectation.

\section{SPACE 1323}

Radiological findings of bacterial pneumonias in patients infected by the human immunodeficiency virus

M. Torres (Presenter), M. D. Martinez-Piazza, M. Molinero, J. J. Muñoz, F. C. O. Orihuela, F. Serrano; Malaga

Purpose: We show our experience in the radiological findings of bacterial pneumonias in HIV positive patients, findings about which little has been published because this pathology is less frequent than others typical opportunistic infections.

Methods: We present a series of 13 male patients, all of them intravenous drug abusers in whom different causative agents were isolated, and two of them presented two concomitant germens. 2 died because the pulmonary infection and the remainder recovered completely. No patient had neither previous pulmonary infections nor other AIDS critera.

Results: We found inferior lobes affected in 8 patients (mostly right and either diffuse or multiple alveolar infiltrates in 5 cases, cavitary lesions were present in 7 cases as an actual parenquimal destruction. Associated pleural effusion was observed in 6 cases. Radiological evolution to normal was found in 10 cases, included those with pulmonary parenchymal destruction, after a prolongate course. These findings lead us to think that bacterial pneumonia in HIV positive patients, characteristically presents a torpid evolution due to an increased aggressivity of causative agents in comparison with non-immunosuppressed hosts in general population and a good response to treatment, with a complete cure in a high proportion of patients.

\section{SPACE 1324}

Thoracic hydatidosis - radiography, CT, MRI, and pathology Walther von Sinner (Presenter); Riyadh

In 25 of 105 patients with hydatid disease (echinococcus granulosus), thoracic hydatidosis was present. Although many publications exist treating the radiological appearance of hydatid disease of the chest, only a few case reports have been published using modern imaging modalities, such as CT and MRI.

The purpose of this exhibit is to demonstrate the different diagnostic signs and characteristics in patients with hydatid disease of the chest correlating radiography with CT and MRI confirmed by FNAB and/or pathology.

The cases include all kinds of hydatid cysts, from the lung parenchyma, mediastinum (including the heart), the pleura, the chest wall.

Complications of thoracic hydatid cysts, such as rupture, infection, pleural involvement, spread and calcifications are also included.

\section{SPACE 1325}

\section{CT manifestations of subacute thoracic histoplasmosis}

Louis H. Wetzel (Presenter), Linda A. Harrison, Errol Levine, Cheng Cho, Jon F. Moran, Arch W. Templeton; Kansas City, KS

Purpose: This exhibit illustrates the varied and often misleading CT findings of thoracic histoplasmosis in children and young adults. 
Methods and Materials: CT examinations of 20 patients, ages 8 months to 34 years, with biopsy or serologically confirmed histoplasmosis were reviewed. Each case was analyzed with regard to size, distribution and character of parenchymal and mediastinal abnormalities and involvement of normal mediastinal structures.

Results: The most frequent finding was non-calcified mediastinal and/or hilar adenopathy $(14 / 20)$ with unifocal masses in 5 cases. Other CT findings included pulmonary infiltrates (4), segmental atelectasis (2), and pulmonary nodules (9). Owing to the nonspecific symptoms and infrequency of pulmonary infiltrates, mediastinal abnormalities were often confused with lymphoma or other mediastinal masses on initial CT evaluation. Mediastinoscopy or thoracotomy was performed for biopsy in $70 \%$ of patients. Increased awareness of the CT manifestations of thoracic histoplasmosis in young persons may enable the radiologist to suggest this diagnosis early on in the course of patient workup. If serological confirmation is obtained, thoracotomy may be avoided in some cases.

\section{SPACE 1326}

\section{CT appearance of panacinar emphysema}

Masahiko Yamagishi (Presenter), Hiroyuki Koba, Seiya Katoh, Masaki Mori, Akira Suzuki; Sapporo

Panacinar emphysema (PAE) is a disease defined pathologically and its clinical diagnosis is difficult. Recently, several studies have shown that computed tomography (CT) is sensitive in detecting emphysema, especially centriacinar emphysema (CAE). The purpose of this study was to characterize the CT appearance of PAE with the corresponding pathologic specimens. We analyzed CT images of 5 individual lobes (right middle lobe, 1 ; left lower lobe, 1 ; right lower lobe, 3 ) with PAE obtained from autopsy or thoracotomy for solitary lung tumor. The lobes were inflation-fixed by Heitzman's method and sliced axially for a CT-pathologic correlative study. One lobe with mild PAE had normal appearance on CT, however, the other four lobes with PAE demonstrated low-attenuation areas (LAA) with various shapes and sizes. One of the LAA's was locally distributed on CT slices, two were dominant in the inner zones, and the other was diffuse.

These CT findings were significantly different from those of CAE as reported in other studies. We concluded that CT is useful in the clinical diagnosis of PAE and its differentiation from CAE.

\section{SPACE 1327}

Primary lung neoplasm calcification demonstrated by CT A. Zidan (Presenter), J.Cáceres, J.M. Mata, E. Castañer, C. Traid, P. Alvarado; Barcelona

Purpose: The presence of calcification in pulmonary masses is considered as a criterion of benignity. Primary pulmonar neoplasms infrequently calcify, and this may lead to misdiagnosis.

Methods and Materials: We reviewed retrospectively the CT of 389 consecutive patients, who underwent lung neoplasm staging, for the presence of calcification. CT was performed with $8-10 \mathrm{~mm}$ slice thickness, $10 \mathrm{~mm}$ interval, and without contrast material.

Results: Calcification was demonstrated in 15 patients $(3,86 \%)$. All were men their mean age was 57 years (range, $35-73$ years). The mean greater diameter was $4,5 \mathrm{~cm}$ (range, $3-9 \mathrm{~cm}$ ).

The histologic diagnosis was squamous cell carcinoma $(n=12)$, oatcell carcinoma $(n=2)$, and adenocarcinoma $(n=1)$.

The calcification was single, dense, and central $(n=2)$, or multiple punctate, or amorphous located centrally $(n=1)$, peripherally $(n=5)$, or diffusely $(n=7)$. One patient had multiple enlarged calcified presumed metastasic lymph nodes. Another 6 patients had densely calcified granulomas and/or granulomatous lymph nodes.

Calcification in lung masses should not be considered a criterion of benignity and such masses should be analyzed as if they were not calcified.

\section{SE \\ SPACE 1328}

Embolization of "high-flow" priapism: 2 cases

Javier Abad (Presenter), Robert Villar, Guillermo Parga, Rafael Fernández, Enrique Garcia-Hidalgo; Madrid

We report 2 cases of "high-flow" or arterial priapism treated with fluid embolic agents.

Both cases were caused by penile trauma.

Selective internal pudendal angiography helped to identify arterio-cavernous fistula. Supraselective catheterization with Tracker catheter was required and embolization was made with Isobutyl-2-cyanoacrylato. The patients were followed with Duplex-Doppler sonography; after the procedure, Doppler revealed no fistula and good flow through the penile arteries. One year later both patients remain asymptomatic.

We think that supraselective embolization with Isobutyl-2-cyanoacrylato is a good treatment in those cases of "high-flow" priapism.

\section{SPACE 1329}

Direct coronal CT in abdominal aortic aneurysm study A. Alvarez Castells (Presenter), S. Pedraza, J.M. Rius Chornet, C. Torrents, A. Segarra, J. A. Jimenez; Barcelona

Several radiological techniques have been used in the diagnosis and presurgical evaluation of the A.A.A.

Ultrasound is the cheapest exploration, but it has well known limitations.

Conventional or digital angiography doesn't visualize wall thrombosis and it's an invasive method.

CT is the most accurate method in A.A.A. evaluation. Measurements obtained on CT correlate precisely with those found at surgery. The lumen size can be differentiated from the adjacent wall thrombosis after intravenous injection of contrast agents. The origin and length of an aneurysm, as well as its relationship to visceral and iliac arteries are well demonstrated. However axial sections are not always able to determinate if renal arteries origin are involved by the aneurysm.

Direct coronal sections allow to visualize in a single scan the whole aneurysm extension, its relationship with renal arteries and the presence of a neck where the surgeon will can operate.

\section{SPACE 1330}

\section{Pre-operative embolisation of Ring's neoplasms}

A. Baldjiisky (Presenter), V. Pantcheff; Pleven

The pre-operative transcatheter embolization of the iliac arteries has become a more and more largely medical therapeutic approach in bladder cancer patients but is not so more used to the patients with pelvic ring's neoplasms. It avoids operative risks makes the operative intervention almost bloodless. We present IO patients with primary neoplasms of the pelvic bones and soft tissues. A great operative risk was considered for 6 of them. An operation was appeared for the 4 patients. After applying preoperative embolisation to the 6 patients, operation was considered possible to 4 of them. During the operation there was no significant bloodless.

\section{SPACE 1331}

Color Doppler ultrasound studies in the evaluation of the neovascularisation of different tumors

I. Bali (Presenter), Zs. Csókási; Szeged

The authors have performed Duplex and color Doppler sonographic examinations on 270 primary or metastatic tumors of various sizes and locations, using ACUSON 128 ultrasound machine with $3.5-5.0-7.0 \mathrm{MHz}$ sector or linear transducers.

The characteristics of the pulse Doppler spectrum, echopattern, vascularisation and blood flow of the tumors were evaluated. The analysis of the parameters is very important in the differential diagnosis of the lesions and in the planning of the necessary (next) examinations; furthermore it is beneficial in the follow-up studies and helps to prove the effectivity of the various therapeutic procedures.

\section{SPACE 1332}

Contrast cine MRI In combination with the centerline analysis: a potential method for estimation of myocardium at further risk

M. S. Beese (Presenter), P.W. Weber, T. Faßbender, M. Reuter, R. Maas, V. Nicolas; Hamburg

32 patients with myocardial infarction (17 chronic, 15 acute) proved by coronary angiography and contrast ventriculography, were investigated by cine MRI after application of Gadolinium DTPA. The systolic wall thickening and shortening fraction for 100 chords were calculated by the centerline method. Wall motion and wall thickening in MRI showed a good agreement with contrast ventriculography $(r \pm .87, p<.005$ and $r=.89, p<.001)$.

Signal enhancement after i.v. application of $0.2 \mathrm{mmol} / \mathrm{kg}$ Gadolinium marked the infarcted myocardium. The necrotic areas showed a passiv systolic inward motion without wall thickening in 9 of 15 patients with acute infarction. Akinesis with lack of wall thickening was detected in 7 of 9 patients in a controlinvestigation after 7 to 26 months.

Conclusions:

1. Cine MRI shows good agreement with cine angiography in determination of regional left ventricular wall motion.

2. Gadolinium DTPA promotes localization and quantification of acute myocardial infarction.

3. The detection of the relation between wall thickening and wall motion by 
contrast MRI may be a good indicator for the identification of myocardium at further risk.

\section{SPACE 1333}

Morphologic definition of arrhythmogenic tissue in the MRI after its functional localization using magnetocardiography

A. Bock (Presenter), M.-C. Dulce, M. Oeff, L. Hennig, S. N. Erné, B. Hamm, K.-J. Wolf; Berlin

Purpose: To study the feasibility of MRI for morphologic localization of arrhythmogenic tissue as defined functionally by magnetocardiographic and invasive electrophysiologic techniques a 3-dimensional orthogonal grid was developed, whose extrathoracic markers could be detected in the MRI $\left(\mathrm{CuSO}_{4}\right)$, Cine-Angiographie (lead) and the magnetocardiogram (inductioncoil) and were arranged within a $5 \mathrm{~mm}$ diameter capsule.

Methods and Materials: In 12 patients with overt Wolff-Parkinson-WhiteSyndrome and preexcitation of the right (2) or left free wall (7) or the posterior septum (3), the MRI of the heart (ECG-triggered Spinechosequence, TE $=12$, $T R=R R$ ) was performed. The functional localization of ventricular preexcitation was defined by MCG (magnetic shielding, 37-channel SQUID-Magnetometer) and electrode catheter position at the side of the accessory pathway (Cine-film). All localization results were related to the constant grid position. Results: EP and MCG localization were comparable with a deviation of $1 \mathrm{~cm}$. Both were projected at the AV groove in patients with free wall accessory pathway (AP) or at the os of the coronary sinus in patients with preexcitation syndrome (PS).

Conclusions: Using a multifunctional precardial grid as a reference, cardiac MRI provides exact morphologic information on arrhythmogenic tissue, which is important for interventional electrophysiology (catheter ablation or antitachycardiac surgery).

\section{SPACE 1334}

Expandable tubular tantalum stents for treatment of arteriosclerotic lesions

F. Camuñez (Presenter), A. Echenagusia, J. Peiro, M. Gonzalez, S. S. Coll, D. Grande; Madrid

We performed a clinical evaluation of strecker stent. Twenty-one patients (23 extremities) with arteriosclerotic stenoses and occlusions of the iliac and femoro-popliteal arteries were treated with 30 stents measuring $4-8 \mathrm{~mm}$ in diameter. Indications were poor results with percutaneous transluminal angiopathy (PTA) in all cases.

In two patients with femoro-popliteal lesions the indication was a limb at risk for amputation.

Follow-up was available in 20 patients (average 7 months; range 1-14 months). On 23 extremities, 20 were patent and two occluded. In the follow-up, clinical grade (Lafontaine) and ankle-brachial indexes improved significantly in 21 extremities ( $100 \%$ in iliac lesions).

The technique of implantation and complications are analyzed.

\section{SPACE 1335}

Abdominal aortic coartaction: treatment with aortic PTA, a case report Enrico Casalini (Presenter); Milano

Abdominal aortic coartaction is a congenital rare vascular disease, characterized by severe stenosis of the abdominal aorta.

Surgical management is complex.

Percutaneous transluminal angioplasty (PTA) is an effective method of treatment for vascular stenosis and obstruction.

We report a successful case of balloon dilatation angioplasty of an abdominal coartaction combined with surgical revascularization of the stenotis right renal artery.

\section{SPACE 1336}

\section{Interventional angioscopy's promises}

J. P. Cecile (Presenter), J. C. Baudrillard, H. Foucart, Ch. Carlier; Lens

But: Since the angioscopy specifies the pathological nature of the arteriopathy, it enables some intravascular procedures. Its development could give way to the "ENDOLUMINAL SURGERY".

Methods and results: Since 1987, the authors have systematically associated the angioscopy with 290 cases of lower limbs arteries angioplasties, including 42 cases of endoluminal procedures. After instruments presentation, the results are described:

- aspiration of three fresh clots unbedded in stenosis previous balloon angioplasty, preventing distal migration;

- one altered clot extraction with dormia cage;

- angioscopy monitoring of four severe stenoses, preventing laceration due to over inflation;
- six balloon catheters correctly driven into the true channel preventing false channel laceration:

- four recanalisations by thermo-laser;

-10 stents insertion under angioscopy control. Later endoscopy demonstrates the evolution is different according to the type and location of the stent;

- successfully extraction of 6 atheromas. One example of every technique is displayed on video tape.

Conclusions: Percutaneous angioscopy looks useful if not necessary previous angioplasty: WATCHING FOR BETTER UNDERSTANDING. It extends recanalisation indications to iliac arteries occlusions ( $12 \%$ of iliac arteritis); femoral arteries procedures are carried out more safely and "à la carte" according to the pathologic lesion: "UNDERSTANDING FOR BETTER TECHNIQUE". Increasing interest of radiologists and surgeons should "ENDOLUMINAL SURGERY" progress.

\section{SPACE 1337}

Histologic examination of specimens obtained with the transluminal extraction catheter during arterial recanalization

M. Chiesura-Corona (Presenter), G.P. Feltrin, G. Thiene, S. Savastano, D. Miotto, A. Torraco, L. Castellan; Padova

Purpose: To evaluate the histology of retrieved material, obtained during arterial recanalization with the Transluminal Extraction Catheter (TEC). Methods: 35 lesions ( 12 stenoses and 23 occlusions) were recanalized with the TEC in 27 patients. At the end of the procedure pathologic analysis was performed on the excised material in 20 cases; both usual and particular stains were used.

Results: Histological examination revealed a whole plaque in one case; in all the others the retrieved material appeared disrupted and grinded. Fibrotic components were evident in all cases $(20 / 20)$, while atheromatous material was demonstrated in 5 cases. Thrombus, both fresh and organizing, was present in almost all specimens $(19 / 20)$; calcium components were found in 6 cases; in 7 cases elastic fibers from tunica media were present.

Conclusions: This analysis indicates that the atherosclerotic plaque grows mainly by deposition and thrombosis, with subsequent fibrotic organization. Recent mural thrombosis is frequently found in complete occlusions $(80 \%)$ also in patients with history of chronic disease, attesting the effectiveness of TEC in recanalizing such long lesions. The presence of fibrotic material, atheromatous components and calcium indicates the capability to remove also material often resistant to conventional PTA. The absence of adventitia confirms the sure intraluminal action of the instrument.

\section{SPACE 1338}

Thrombogenicity of intravascular stents in experimental studies Yeon Hyeon Choe (Presenter), Jae Hyung Park, Joon Koo Han, Man Chung Han, Chu-Wan Kim; Kyonggi-do

Twenty-seven Gianturco type self-expandable stainless steel intravascular stents were placed into the infrarenal abdominal aortas $(n=17)$ stents were placed into the infrarenal abdominal aortas $(n=17)$ and inferior venae cavae $(n=10)$ in 10 mongrel dogs. In eight aortas, stents were delivered after balloon catheter-induced denudation of intima and in four out of the eight aortas, stents were also placed into the normal sites to compare neointimal thickness of the normal and denuded sites.

Angiography, light microscopic and scanning electron microscopic examinations were performed after 1 week $(n=1), 2$ weeks $(n=6), 3$ weeks $(n=5)$ and 6 weeks $(n=2)$. The patency rate and thrombosis rate were $100 \%$ and $35 \%$ in aortas and $100 \%$ and $60 \%$ in inferior venae cavae.

The aortic stent wires were covered with thin fibrinous membrane, blood cells and scanty myointimal cells after 1 week. Nearly complete transparent neointimal covering was found in aortas and caval veins after 2 weeks. There was no significant difference in intimal thickness between the denuded and normal aortas after 2 weeks $(p=0.51)$ and 3 weeks $(p=0.56)$. In four dogs with caval vein stents, there were evidences of hemorrhagic infarction in lungs.

The thrombosis rate of 12 stents with stent-artery ratio (SAR) 1.5 or higher was $41 \%$, while there was no thrombus on the four stents with SAR 1.2 or lower except one specimen after I week. The high SAR of aortic stents may be a cause of thrombosis due to prolonged damage to intima and media. It is suggested that stents with SAR 1.2 or lower are more suitable. The high thrombosis rate of inferior venae cavae may be related with slow blood flow.

\section{SPACE 1339}

MR-anglography of lower limb arteries as follow-up after PTA B. M. Cramer (Presenter), E. Schlegel, M. Boos, G. Laub; Wuppertal

Purpose: Comparison of conventional and MR-Angiography regarding stenoses and occlusions.

Methods: 160 procedures of either modality were performed on arteriosclerotic patients prior and post PTA. We used 3D-Fisp-sequences with re-phased and de-phased subtracted images on a Siemens 1.5 T-Magnetom. 
Results: There was excellent correlation of conventional and MR-Angiography. Length of occlusions, degree of stenosis and number of collateral vessels were visualized in all situations except one: Short and high grade stenoses may be visualized as occlusions because of the limited local resolution. All intimal tears were depicted by MR-Angiography.

Conclusions: MR-Angiography proved clinically useful as regional, non-invasive follow-up after PTA and may vary after vascular surgery as well. Lack of spacial resolution was very well compensated by the high flow sensitivity of the method.

\section{SPACE 1340}

Congenital anomalies of inferior vena cava and left renal vein: non-invasive diagnosis with duplex-Doppler sonography L. E. Derchi (Presenter), G. Crespi, F. Pretolesi, A. D'Amica, L. Oliva; Genova

The sonographic findings observed in five patients with congenital anomalies of the inferior vena cava and left renal vein are presented. We met two patients with duplication of the inferior vena cava, two with transposition of the vessel, and one with retroaortic left renal vein. In four cases the diagnosis was confirmed through demonstration of venous flow within the imaged anomalous vascular structures using duplex Doppler equipment. The diagnostic capabilities of sonographic imaging and Doppler analysis of blood flow allow a non-invasive diagnosis of these vascular anomalies. In our opinion, contrast studies are needed only when retroperitoneal structures are difficult to evaluate by ultrasound due to obesity and/or overlying abundant intestinal gas.

\section{SPACE 1341}

Cine-MR in the determination of left ventricular volumes and heart function: comparison of different calculation procedures and measurement techniques

M.-C. Dulce (Presenter), K. Friese, A. Albrecht, B. Hamm, K.-J. Wolf; Berlin

Introduction: The purpose of our study is to test the variability and reproducibility of left ventricular volumes and functional parameters using Cine-MR. The introduction of Cine-MR as a routine procedure will be discussed.

Methods: 16 volunteers were evaluated by MRI using a 1.5 Tesla equipment. The left ventricle was shown in different planes. We used a gradientecho-sequence $(T E=12 \mathrm{~ms} ; \mathrm{FL}=60 ; \mathrm{TR}=\mathrm{RR})$. The calculation procedures (monoplane, biplane, 3-dimensional, reconstruction by adding the areas of each slice) were tested for their concordant results, The images were evaluated by two independant observers. The reproducibility was tested by follow-up examinations.

Results: The 3-dimensional reconstruction of the left ventricle as well as the biplane calculation procedure showed a very low standard deviation (SD) of the means (stroke volume: $\mathrm{SD}<16 \mathrm{ml}$; ejection fraction: $\mathrm{SD}<2 \%$ ). The standard deviation of the monoplane calculations were higher (SV: SD $20 \mathrm{ml}$; EF: SD 5\%). Control examinations showed well reproducible $(r>0.75$, $p<0.001$ ). A low interobserver variability was observed (\%variability $<5 \%$ ). Conclusions: Cine-MR is a very accurate procedure in the assessment of left ventricular volumes and functional heart parameters. It should not only be used for follow-up studies but also for routine applications.

\section{SPACE 1342}

Computed tomography and magnetic resonance imaging in pericardial and retrosternal adhesions subsequent to cardiac surgery

Olov Duvernoy (Presenter), Torsten Malm, Karl-Äke Thuomas, Sven G. Larsson, Hans-Erik Hansson; Uppsala

Abstract: The visibility of the pericardium as well as the space between the dorsal aspect of the sternal surface and the pericardial surface was determined in CT and/or MRI in ECG-gated spinecho (SE) and gradient echo (GE) sequences. Seventeen patients who had undergone cardiac operations, and were admitted for cardiac reoperation were investigated with CT and/or MRI prior to sternal reentry. Five patients were investigated with CT, ten with both CT and MRI and two with MRI only. Retrosternal adhesions were classified as present or absent, intrapericardial adhesions were classified; absent, minimal, moderate or severe at re-operation. A similar classification was applied to the findings at CT and MRI. In 14/15 patients, the findings at CT were in accordance with those found at operation regarding postoperative retrosternal extrapericardial adhesions in the cranial retrosternal space, and in $12 / 15$ in the caudal retrosternal space. CT could not detect intrapericardial adhesions. MRI was sensitive to metal artefacts from sternal sutures in both sequences and could therefore not be used to detect postoperative retrosternal extrapericardial adhesions. MRI with ECG-gated SE sequences confirmed intrapericardial adhesions in 44/57 locations. Absence of intrapericardial adhesions revealed at operation was shown in 13 locations.

\section{SPACE 1343}

Practical intravascular ultrasound: evaluation of arteriosclerosis and arterial interventions

Christopher E. Engeler (Presenter), Claudia M. Engeler, Joseph W. Yedlicka, Jr., David W. Hunter, Janls Gissel Letourneau, Wilfrido R. Castaneda-Zuniga, Kurt Amplatz; Minneapolis, MN

Purpose: Demonstration of the practical application of intravascular sonography in the diagnosis and management of arteriosclerosis.

Methods and Materials: High-resolution intravascular catheter-based ultrasound (US) of 40 patients was performed with a $6.6 \mathrm{Fr} 20 \mathrm{MHz}$ probe (Diasonics Inc. Milpitas, CA and Boston Scientific Corporation, Watertown, MA) and correlated with angiography in the aorta, iliac arteries, and arteries of the lower extremities. Intravascular US was used to monitor revascularization procedures including angioplasty, atherectomy, and stenting.

Results: A step-by-step approach to the application and the unique abilities of this new technology are illustrated in 1 . the diagnosis of vascular disease not evident by angiography; 2 . accurate calculation of stenotic area; 3 . sonographic characterization of pathologic deposits; and 4. effects in interventional procedures with special emphasis on intimal flaps and dissections after angioplasty.

Conclusions: The broad spectrum of arteriosclerotic disease is within the reach and resolving power of catheter-based intravascular US. We believe the US appraisal of arterial wall characteristics will ultimately aid in the choice of vascular interventional strategies in addition to reducing the amount of angiographic contrast and fluoroscopy needed to monitor procedures.

\section{SPACE 1344}

Colour Doppler ultrasonography in atherosclerotic obstructive disease of lower extremities

László Erdélyi (Presenter), Mózes Péter, Judit Nagy; Debrecen

The lower extremities of 50 patients with atherosclerotic obstructive disease were studied by both color Doppler sonography and arteriography. Stenosis and obstructions of main arteries and collateral circulation were detected by colour Doppler sonography.

In the majority of cases this method can be used instead of arteriography. The signs of changes in atherosclerotic obstructive disease and the advantages and handicaps of this technique are summarised in the poster.

\section{SPACE 1345}

Transcatheter closure of patent ductus arteriosus with original transvenous technique

V.I. Prokubovski, S. M. Kolody, S. V. Saveliev, V. E. Verin, A. Ya. Farber (Presenter); Hannover

The patent ductus arteriosus (PDA) is closed with conical-shaped device which consists of polyurethane foam mounted on stainless steel frame. The plug is delivered into the duct through the transvenous Teflon sheath. Since 1981 till 1991 the procedure was undertaken in 262 pts. Permanent complete duct closure was achieved in $248(94,7 \%)$. In 7 cases the occlusion was partial. The complications occurred in $10 \mathrm{pts}$. There were migration of the plug into the abdominal aorta in 4 pts, wrong fixation of the device in the left subclavian artery origin $-2 \mathrm{pts}$, preliminary fixation of the plug on the wall of the descending aorta $-1 \mathrm{pt}$, migration of the plug into the pulmonary artery bed -3 pts. 7 of these failures were treated surgically.

After 10 years of experience we consider the developed technique as very effective, safe and applicable in more than $80 \%$ of patients with PDA in the age older than 2,5 years.

\section{SPACE 1346}

Pitfalls in the ultrasonography diagnosis of lower-extremity deep venous thrombosis

J. Fernández Sánchez (Presenter), W. Bücklein, E. Klotz; Augsburg

Real-time B-mode duplex Ultrasonography (US) is a highly accurate, noninvasive method for the diagnosis of lower-extremity deep venous thrombosis (DVT). Knowledge of the potential vascular malformations and variants in the pelvis and legs is essential for the correct interpretation of US. Familiarity with such variations can provide a correct diagnosis and obviate the need of venography. The aim of this study was to evaluate these vascular anomalies with US using venography for correlation.

In 145 patients (169 extremities) with clinically suspected DVT of the lower extremity a venography and an US were performed. In these examinations we have found some venous anomalies, such as bifurcation of the femoral or popliteal vein, and several pathological conditions, such as venous aneurysms and arteriovenous fistulas, that may lead to diagnostic problems in patients with suspected DVT. Other potential diagnostic pitfalls of US are small nonocclusive thrombi, extraluminal compression of the deep veins caused by soft 
tissue abnormalities, positive compression US-test in some fresh thrombi, which may be slightly compressible, non-adequate performance of compression US in obese patients and other possible interpretative errors caused by technical limitations of the study. This scientific exhibit provides illustrations and discussion of these venous anomalies and pitfalls.

\section{SPACE 1347}

A study of the assessment of coronary flow by videodensitometry in the closed chest canine model: validation by microspheres

G. Finet (Presenter), M. de Lorgeril, M. Ovize, D. Revel, M. Amiel; Lyon

The aim of this study was to validate with radiolabelled microspheres the quantification of the different coronary flow rates by studying contrast density obtained by digital subtraction angiography (DSA) in an experimental canine preparation.

Methods: 2 sequences [rest state/during DPD $(0.75 \mathrm{mg} / \mathrm{kg}$ during $4 \mathrm{~min}$ and followed by $0.1 \mathrm{mg} / \mathrm{kg} / \mathrm{min})]$ in 5 intact Beagle dogs have been realized providing for each dog 2 coronary flow reserve (CRF: hyperemic flow/basal now) according to 2 methods. 1. Regional myocardial blood flow (RMBF) was measured with microspheres ( 4 radionuclides) by the reference sample method (flow results: $\mathrm{ml} / \mathrm{min}$ per $\mathrm{g}$ ). 2 . The time-density curve (TDC) was obtained within a region of interest chosed over the part of myocardium supplied by the left circumflex artery in the consecutive images by DSA. The TDC remaining was fitted to a gamma-function and 3 measurements were calculated: the aera under the curve (A), the peak density (PD), the mean transit time (MTT). Index of CFR was computed as the ratio of hyperemic to basal measurements for each of these methods and was compared with index assessed by microspheres. Results: 9 CRF have been calculated 3,47 1,67 (mean \pm SD). The correlation between the 3 index of CRF determined by videodensitometry and the CFR measured with microspheres are for $\mathrm{A} \mathrm{r}=0.97(\mathrm{y}=.77 \mathrm{x}+.58)$, for DP $r=0.92(y=.7 x-.11)$, and MTT $r=0.95(y=.72 x+.52)$.

Conclusions: These results indicate that the 3 videodensitometric index can be used to accurately assess changes in myocardial perfusion.

\section{SPACE 1348}

CT-Fluoroscopy (CTF): role in intravascular diagnostic and interventional procedures

L.F. Frigerio (Presenter), C. Spreafico, A. Marchiano, I. Spagnoli, F. Garbagnati, M. Milella, B. Damascelli; Milano

Purpose: To assess the usefulness of a combined technique in the catheterization of vascular structures in patients undergoing diagnostic procedures or interventional maneuvers in the vascular system.

Methods and Materials: The patients lay on the CT table and a mobile $\mathrm{C}$-armed digital subtraction $\mathrm{X}$-ray system provides the fluoroscopic control of the entire procedure. 57 arterial portographies were performed by injecting the contrast medium directly into the superior mesenteric artery, carrying out the entire procedure in the $\mathrm{CT}$ room. 1 procedure was performed to retrieve a piece of a non-radiopaque catheter lost in the bloodstream, which CT easily identified. 5 percutaneous embolizations of artero-venoùs fistulas were performed using CTF, so that CT could localize the emboli and give a real-time assessment of the reduction of the vascular pattern. In 10 cases of multifocal hepatocellular carcinoma, a percutaneous chemoembolization was carried out using Mitomicin C microcapsules and Lipiodol.

Results: All procedures were safely carried out. CTF provided little discomfort to the patient, less radiation exposure to both the patient and the radiologic staff, less contamination when the procedure had to be performed on HIV-positive subjects.

\section{SPACE 1349}

Clinical usefulness of rotational stereo digital angiography for the diagnosis of aortic dissection

Kazuhito Gemma (Presenter), Toru Ohya, Hiroyuki Tajima, Koichiro Ito, Tatsuo Kumazaki, Kinichi Ebata; Tokyo

Purpose: To evaluate a clinical usefulness of rotational stereo digital angiography (RSDA) for diagnosing aortic dissection, particularly for the detection of intimal tear sites.

Methods and Materials: Twelve cases with aortic dissection of opacified false lumen were examined by RSDA. Thoracic, abdominal, pelvic aortographies were performed using 12 inch I.I.-TV system. The $X$-ray apparatus rotates transversely at a speed of 2 seconds during 180 degrees when pulse mode exposures are done. Through digitation images are displayed real time on two CRTs (matrix $515 \times 512$ ) with an anlge of 5 different degrees, so that observers can see three-dimension angiograms from any angle during 180 degrees.

Results: In 12 patients, a total number of 48 intimal tear sites could be detected. They were 2 in ascending aorta, 2 in aortic arch, 17 in descending aorta, 19 in abdominal aorta, and 8 in pelvic artery. An adequate angle for the demonstration of each lesion could easily be obtained following just a single injection of contrast medium.
PTA of renal artery, the risk of the procedure in relation to morphological type of stenosis

S. Goryń (Presenter), O. Rowiński, B. Pruszyński, M. Szostek, A. Kulesza; Warszawa

The main aim of the study is evaluation of the prognosis of PTRA in relation to morphological type of the renal artery stenosis. The material consists of 250 procedures. Our experience allows for the conclusion that the most important risk factors are location and extent of the stenosis.

\section{SPACE 1351}

Percutaneous transluminal implantation of the Guenther caval filter experience in 22 patients

F. Grabenwöger (Presenter), W. Dock, V. Metz, K. Eibenberger, H. Magometschnig; Vienna

Purpose: The goal of this study was to determine the success rate as well as the complication rate of the Guenther caval filter.

Methods: In the last 3 years, 24 patients were referred to our institution for placement of a caval filter. The indication for operation was either recurrent pulmonary emboli or prophylaxis against emboli from pelvic or deep vein thrombosis. In 2 cases implantation of a caval filter was not attempted due to a low take-off of the renal veins. In the remaining 22 patients a Guenther caval filter was successfully placed. Seventeen patients could be followed with a mean follow-up of 14 months.

Results: In no patient was there clinical evidence of new pulmonary emboli after successful implantation of the caval filter. An occlusion of the inferior vena cava was found in one patient ahd a caudal dislocation of the caval filter in 6 patients. In one patient one of the fixation feet of the caval umbrella broke. Conclusions: This study demonstrated that the Guenther caval filter has both a low complication rate and a high success rate with regard to the prevention of recurrent pulmonary emboli.

\section{SPACE 1352}

CT analysis of vascular wall during the active phase of Takayasu's aortitis

H. Hayashi (Presenter), N. Katayama, R. Takagi, T. Ichikawa, T. Kumazaki, K. Ebata; Tokyo

The vascular wall of patients during the active phase of Takayasu's aortitis (TA) was investigated on CT.

Four female patients, aging 12-22 years, were examined. Final diagnosis of TA was made by both clinical findings and aortography. The vascular wall thickness and the ratio of the thickness to inner circumference of common carotid arteries and thoracic aorta were calculated. These items were compared with normal anatomic values. Findings of the vascular wall before and after the administration of contrast medium were also examined.

The vascular wall thickness and the ratio to inner circumference were markedly increased in all cases. On plain $\mathrm{CT}$, the vascular wall clearly distinguished from the vascular lumen by similar to or higher density than that of the muscle. Following contrast medium injection, the wall showed a "double ring-like pattern"; a poorly enhanced inside ring and a densely enhanced outside ring. The inside ring is considered to be mucoid or gelatinous swelling of the intima, while the outside ring be an active medial and adventitial inflammatory change with neovascularization.

These CT findings, which have not been reported previously, will be useful for the early diagnosis of TA.

\section{SPACE 1353}

\section{Experience in low-speed rotational angioplasty} M. O. Cizmeli, E. T. Ilgit (Presenter); Ankara

Arterial occlusions of the lower extremities are not amenable to balloon angioplasty (PTBA) alone. The low-speed rotational transluminal angioplasty catheter system (ROTACS) is a recently available device for recanalization prior to PTBA. Heavily calcified occlusions and the presence of a large collateral originating proximal to the occlusion, constitute limitations for ROTACS. To avoid the entry of rotating catheter to the collateral, tip of the catheter is placed just proximal to the occlusion and the hard tip of a $0.020^{\prime \prime}$ guide-wire is used to create an inlet by repeated small, probing movements carefully. Afterwards the tip of the ROTACS catheter is engaged in this inlet and started to rotate at low speed. When ipsilateral approach is not possible as there is no sufficient distance to place an introducer sheath between the puncture site and the occlusion, contralateral antegrade approach is considered. Follow-up angiographic examinations of the successfully recanalized and dilated 16 occlusions in 15 patients up to one year revealed $87.57 \%$ patency rate. We conclude that, creation of a pilot inlet and cross-over 
recanalization will increase the number of candidates for the recanalization with ROTACS prior to PTBA.

\section{SPACE 1354}

Magnetic resonance tomography (MRT) In congenital defects of the left pericardial sac

W. Judmaier (Presenter), I. Gaßner, K. Wicke, I. Hammerer; Innsbruck

A. We report the findings in 4 patients with left sided pericardial defects, and compare the value of MRT with other imaging modalities.

B. Cardiac MRT was performed on a $1.5 \mathrm{~T}$ system using multiplanar ECG triggered T1 weighted Spin Echo Sequences. The appearance of the pericardial sac and anomalies in position and shape of the cardiac chambers were evaluated.

C. In 3 cases with complete defects an abnormal position of the heart, a partial enlargement of the left atrial appendage, and a marked prominence of the pulmonary artery were demonstrated. In one case with partial defect MRT showed the herniation of the left ventricle through the apical pericardial gap. D. Since in children with little epi- and pericardial fat layers the pericardial sac is not always visible with radiological methods, we have to rely on indirect signs ot assess its integrity. Whereas the total absence of the left pericardium is considered to have little clinical effect, partial absence in the apical region can be fatal. The ringlike constriction of the cardiac chamber, so far described in seven cases only upon autopsy, was seen for the first time in vivo on MRT. This 'myocardial strangle' sign should be noted as evidence of a life threatening disease. The diastolic compression of the coronary arteries however, could only be visualized by angiography.

\section{SPACE $\underline{1355}$}

Quantitation of cardiac valvular regurgitation with ultrafast CT scanning Tatsurou Kaminaga (Presenter), Hiroaki Naito, Seiki Hamada, Satoshi Imakita, Naoaki Yamada, Tadashi Nakanishi, Yoshiaki Hirose, Makoto Takamiya; Osaka

Purpose: A new method with ultrafast CT scanning to quantify cardiac valvular regurgitation is proposed.

Methods: Forty-two patients aged from 15 to 71 years (average 48 years) were examined with contrast UFCT. Twenty patients had mitral regurgitation and 10 patients had tricuspid regurgitation. Multislice cine mode UFCT scanning was performed to measure ventricular output with modified Simpson's method. Ventricular output (VO) was defined as follows: $\mathrm{VO}=$ stroke volume $\mathbf{x}$ heart rate. Flow mode UFCT scanning with bolus contrast injection was applied to measure cardiac output (CO) from time-density curve of the pulmonary artery and ascending aorta using Stewart-Hamilton's formula. Regurgitant fraction (RF) was calculated as follows:

$\mathrm{RF}=(\mathrm{VO}-\mathrm{CO}) / \mathrm{VO}$

RF value was compared to the grade of regurgitation measured with cine angiography and/or Doppler ultrasonography.

Results: Regurgitant fractions derived from UFCT scanning favorably correlated to the grade of mitral and tricuspid regurgitation measured with cine angiography and/or Doppler ultrasonography.

Conclusions: Contrast UFCT could be used for precise quantitation of valvular regurgitation with combination of volumetry and densitometry. Furthermore, this method could separately measure the regurgitation of right and left side of the heart.

\section{SPACE 1356}

Usefulness of MRI in the field of cardiovascular pathology - a retrospective study

B. Kastler (Presenter), Ph. Germain, A. Livolsi, A. Gangi, R. Allal, J.L. Dietemann, A. Sacrez, A. Wackenheim; Strasbourg

Aim: In order to assess the practical value of spin echo MRI as function of various indications in the field of cardiovascular (CV) applications, we rewieved the contribution of the MR results for the clinical management of 550 patients studied from 1986 up to April 90. All examinations performed for research protocol were excluded (600 pts).

Methods: A 3 grade score was attributed to each exam according to the relevance of the MR finding with regard to the clinical question:

1 = no significant data provided, 2 = additional clinically significant information \& $3=$ outstanding new information solving diagnosis problems or therapeutic decisions.

Results: In our center, patient referred for CV-MR (protocol excluded) represented $3 \%$ of all MR exams (based on either echographic, X-rays or clinical findings). 255 observations have yet been rewieved. The list below carry back the number and $\%$ of indications and the corresponding retained diagnostic score:

\begin{tabular}{lccccc}
\hline Pathology & N & $\%$ & 1 & 2 & 3 \\
\hline Great vessels pathology: & 63 & 25 & $3 \%$ & $17 \%$ & $80 \%$ \\
Intra/extra cardiac masses: & 43 & 17 & $7 \%$ & $33 \%$ & $60 \%$ \\
Pericardial disease: & 30 & 12 & $14 \%$ & $60 \%$ & $26 \%$ \\
Congenital abnormalities: & 45 & 18 & $17 \%$ & $39 \%$ & $44 \%$ \\
Other cardiac pathology: & 74 & 29 & $9 \%$ & $44 \%$ & $47 \%$ \\
\hline
\end{tabular}

Conclusions: MRI is mainly helpful for managing great vessels problems, suspected cardiac masses, pericardial diseases and congenital heart abnormalities. Other clinical applications are presently less important although very useful answers were punctually provided in many difficult cases when routine procedures appear insufficient.

\section{SPACE 1357}

Phlebographic diagnostic of complications caused by dlalysiscatheter using the digital radiographic system

H. Klengel (Presenter), Sabine Schuster, R. Verwiebe, R. Schuster; Göttingen

The diagnostic of complications caused by dialysiscatheter using the digital spot imaging and the digital subtraction angiography method is being evaluated.

In 7 patients suffering from complications caused by dialysiscatheter positioned in the vena cava superior, technical and diagnostical assessments are being made. While minimizing the total amount of non-ionised contrast medium $(5$ to $10 \mathrm{ml} 300 \mathrm{mg} \mathrm{J} / \mathrm{ml}$ applicated and reducing the flow-rate (2 to $6 \mathrm{ml} / \mathrm{sec}$ ), these proceedings enables the demonstration of thrombogenic clot in the immediate vicinity of the catheter, thrombogenic clot directly attaching to the catheter, or early registration of collateral circulation signalling reflux obstacles.

\section{SPACE 1358}

Role of transesophageal echocardiography (TEE) and MRI for non-rheumatic aortic regurgitation: initial experience

Kazunori Kuroki (Presenter), Toshio Yamaguchi, Azuma Kitagawa, Tohru Ishikawa; Kawasaki

Purpose: It is difficult to clarify the precise cause of aortic regurgitation (AR) by conventional imaging modalities. The purpose of this study is to investigate the utility of TEE and MRI for the evaluation of non-rheumatic AR.

Methods and Materials: An analysis was made 5 patients with acute and severe AR. Transthoracic echocardiography (TTE) and angiogram were performed in all 5, TEE in 4, and MRI in 4. TEE was performed with color-doppler flow. MRI was obtained with $1.5 \mathrm{~T}$ on transverse and coronal images.

Results: TTE failed to identify the precise cause of AR, but demonstrated pouch formation of non-coronary cusp (NCC) in 2 patients. In 4 patients TEE disclosed aortic valve prolapse (AVP) manifested by 2 major findings: non-visualized coaptation of aortic valve and eccentric regurgitation flow with color-doppler in diastole. The pouch formation of NCC, due to Takayasu's arteritis and clinically diagnosed Behçet disease, was visualized more clearly on both TEE and MRI than TTE. MRI revealed invagination of the pouch into the ventricular septum in 2, and localized ascending aortic aneurysm in 1 . Surgery was performed in 2 patients, confirming AVP caused by myxomatous degeneration and aortic aneurysm.

Conclusions: Our preliminary results suggest that TEE may be the modality of choice to clarify the etiology and site of non-rheumatic AR. MRI offers a valuable information about the aortic root, especially on coronal images.

\section{SPACE 1359}

Color Doppler ultrasound of venous valvular incompetence - correlation with clinical and phlebographic findings

Ch. Landré (Presenter), Ch. v. Itter, Th. Harder; Bonn

Aim of this study was to compare color Doppler ultrasound and ascending phlebography in diagnosis of venous reflux in cases of valvular incompetence. 25 patients ( 9 male, 21 female) who were $17-76$ years (mean, $52.5 \pm 16.7$ ) with clinical signs of venous incompetence examined using color Doppler ultrasound and ascending phlebography.

40 examinations were carried out by color Doppler ultrasound. A reflux of the great saphenous vein was detected in 17 cases, of the small saphenous vein in 12 cases and of the femoral vein in 5 cases. 25 investigations were correlated with ascending phlebography. In all cases an excellent correlation was found obtained by both modalities. However, dynamic manoeuvres in color Doppler ultrasound contributed to a higher degree of confidence in diagnosis. 12 examinations by color Doppler ultrasound were correlated with clinical findings. There were no significant differences between the diagnoses obtained by color Doppler ultrasound and the clinical findings. 
Our results indicate, that color Doppler ultrasound is an effective tool in diagnosis of venous valvular incompetence. There is a high degree of correlation between the results of color Doppler ultrasound and ascending phlebography.

\section{SPACE 1360}

Archltecture of cutaneous vascular network by postmortem microangiography

Heinz-Jakob Langen (Presenter), C.M. Breiden-Langen, A.Thron, S. Handt, M. Körber; Aachen

Purpose: Demonstration of the cutaneous vascular architecture by microangiography.

Methods: A suspension of barium sulphate was injected into the main arteries of nine amputated lower limbs. Skin sections of $2 \mathrm{~mm}$ thickness were obtained from the feet. Subsequently, these sections were fixed in formalin, embedded in paraffin and than exposed to $\mathrm{X}$-rays on high-resolution photographic plates. Slices of $5 \mu \mathrm{m}$ thickness from these paraffin blocks were cut for histologic examination and correlated to microangiography.

Results: In comparison to histology, microangiography visualizes cutaneous vascularisation in a greater volume. For this reason, microangiograms provide a nearly threedimensional overview of microcirculatory anatomy. Capillary loops in the papillae, the sub-papillary plexus, the glandular components (with the capillaries surrounding the sweat glands), the candelabra arterioles and the descending venous system in the cutis could be demonstrated over a prolonged course. Direct comparison between microangiographic and histologic pictures of the same tissue slice helps to differentiate artifacts from lesions.

Conclusions: This method enables a comprehensive understanding of the cutaneous microcirculation of the living skin under normal, pathologic, and experimental conditions. Further studies are needed to evaluate its clinical significance.

\section{SPACE 1361}

Carbon dioxide gas perfusion of sapphire probe in laser recanalization of peripheral artery occlusions

Hannu Manninen (Presenter), Xiaoming Yang, Pekka Matsi, Matti Suhonen; Kuopio

Carbon dioxide $\left(\mathrm{CO}_{2}\right)$ gas has proven to be a safe intra-arterial contrast agent for angiography of peripheral arteries. Experimental studies on human cadaveric vessels have shown carbon dioxide gas perfusion of sapphire probe to provide more effective laser ablation of artery atheroma than conventional saline perfusion. This study is undertaken to evaluate utility of carbon dioxide gas perfusion of the sapphire probe in clinical laser recanalization lower extremity artery occlusions.

A $1.064 \mu \mathrm{m}$ Nd-YAG laser source (SLT CL60) with a $2.2 \mathrm{~mm}$ sapphire round tipped probe (SLT Inc) is used for lasing. Perfusion of the probe with medical $\mathrm{CO}_{2}$ gas at flow rate $0.51 / \mathrm{min}$ is performed through a gas delivery cartridge of the laser source. Probe perfusion is limited to the time when the laser source is activated, not more than 15 seconds at a time. Lasing procedure is done under digital fluoroscopy. Carbon dioxide gas, as a negative contrast agent facilitates vessel delineation and controlling of progress of the procedure by using digital road mapping.

Results of the technique in clinical laser angioplasty are presented and the benefits and limitations of the procedure discussed.

\section{SPACE 1362}

Complete arterial obstructions of the lower limbs: the place of percutaneous atherectomy

P. Maquin (Presenter), H. Rousseau, M. Levade, E. Bloom, B. Escude, F. Joffre, J. J. Railhac; Toulouse

Purpose: Evaluation of the Simpson catheter in the treatment of complete arterial obstructions.

Methods: Seventeen patients ( 12 men, 5 women, 51 to 83 years old), presenting with iliac $(n=1)$ and femoro-popliteal $(n=16)$ symptomatic occlusions (mean length: $23 \mathrm{~mm}$ ), were treated by atherectomy. A previous recanalization with balloon angioplasty was necessary in 4 patients, along with a complemental dilatation in 14 cases.

Evaluation included repeated clinical and doppler examinations, angiography (6 months after or in case of clinical worsening).

Results:

- Initial results: disappearance of the claudication was noted in all patients with a mean 0,4 increase in the ankle-arm-index. A residual stenosis lower than $30 \%$ was noted in 16 cases (the severity of the residual stenosis was lower when the catheter used was a 9 french, and when the lesion was short and non calcified. Seven mild dissections were noted. One distal embolization occurred without sequelae.

- Mid-term results: clinical and doppler improvement remained stable in 15 patients with a maximum follow-up of 32 months (mean: 17,3 months); one restenosis at four months; one death, non related to the arterial disease, at 6 months. Atherectomy along with a conventional angioplasty seems to be a safe and effective treatment of short femoro-popliteal obstructions.

\section{SPACE 1363}

\section{Angiographic diagnosis of acute Intestinal ischemia}

R. Dalla Valle, C. Marcato (Presenter), E. Banchini, L. G. Villani, U. Ugolotti; Parma

The diagnosis and treatment of acute mesenteric ischemia is still a debated and often unsolved problem. In case of intestinal infarction, angiography enables to distinguish occlusive from non-occlusive ischemia and can define nature and position of a lesion as well as the conditions of the distal vascular bed. Furthermore angiography represents an important method for both instrumental and pharmacological treatment. In the last decade we recorded in our Institute 40 cases of acute mesenteric ischemia. Nine patients underwent to global aortography and selective angiography of the superior mesenteric artery (SMA); in 5 cases the examination was carried out by traditional technique (Dos Santos), whereas in 4 patients we performed digitalized angiography. The angiographic details of our cases are shown in the poster. We diagnosed in 4 cases embolism and 2 cases thrombosis of SMA main trunk, which later were surgically treated. One case of embolism of the distal branches of SMA and 2 cases of functional ischemia were successfully treated both by local and systemic conservative therapy. In this latter cases only the traditional angiography permits to display the morphology of the distal splanchnic vascular bed. In conclusion we believe that, in the angiographic diagnosis of the acute intestinal ischemia, it is still fundamental to combine traditional and digitalized angiographic techniques in order to supply a detailed map of the splanchnic circulation and therefore to suggest the most suitable therapeutic approach.

\section{SPACE 1364}

Selective arterial embolization of symptomatic renal angiomyolipoma in a patient with tuberous sclerosis

A. Mariscal (Presenter), J.Dominguez, C.Sancho, A. Martinez, X. Montaña; Barcelona

Angiomyolipomas (AML) are benign tumors that commonly occur in association with Tuberous Sclerosis (TS) (60-80\%) and in these cases they are generally bilateral and multiple. The majority are asyntomatic, but they can present themselves clinically with pain, a palpable mass and hematuria. Today, thanks to ecography and CAT it is possible to arrive at a correct diagnosis in almost all cases. Symptomatic AML's, particularly in patients with TS should be treated in a conservative way, in order to preserve intact as much of the normal renal parenchyma as possible. Herag we present a case in which we have employed selective renal arterial embolization to achieve this aim.

Case Description

A 24 year old woman with traces of TS, had complained of left flank pain for one month. An analysis detected anemia which was the motive for further examinations (I.V.U., Ecography, CAT) which enabled a diagnosis of multiple intra-abdominal AML within the context of TS. A large AML was seen in the lower pole left kidney. Later we carried out a diagnostic and therapeutic selective renal arteriograph of the mass. For this we used a 5.0-F catheter and Gelfoam and Coil as the embolization materials. A control cat three months later, revealed a large reduction in the size of the tumour. The clinical follow-up did not show any complication. The patient remaining asymptomatic with good renal function.

\section{SPACE 1365}

Duplex Doppler ultrasound: an easy way to detect alterations in renal blood flow non-invasively

I. Mastorakou (Presenter), J. Firth, D. Young, C. Garrard, D. Lindsell, J. Ledingham; Oxford

Purpose: Duplex Doppler Ultrasound as a method to assess alterations in renal blood flow in intensive care patients after intravenous infusion of a vasoactive agent such as dopamine.

Methods and Materials: 20 persons, including 6 healthy volunteers, 6 patients with impaired renal function and 8 patients with normal renal function were examined. The peak systolic, end diastolic and mean velocities were measured from a waveform of an interlobar artery. Pulsatility and Resistance Indices (PI, $\mathrm{RI}$ ) were then calculated before and after the infusion of dopamine at doses of 2.5 and $5.0 \mu \mathrm{g} / \mathrm{kg} / \mathrm{min}$.

Results: The mean values of both indices were reduced during the infusion of the $2.5 \mu \mathrm{g} / \mathrm{kg} / \mathrm{min}$ dose and return to baseline levels during the infusion of the $5.0 \mu \mathrm{g} / \mathrm{kg} / \mathrm{min}$ dose. The reduction in the mean values of PI was statistically significant in all subgroups $(\mathrm{p}<0.01$-paired $t$-test). It has already been shown that dopamine at $2.5 \mu \mathrm{g} / \mathrm{kg} / \mathrm{min}$ increases renal perfusion in healthy subjects, so a reduction in indices was expected. However, similar changes produced in patients with and without renal impairement provides some new evidence that 
this also occurs in renal impairement. It was also demonstrated that the mean values of the indices were significantly higher in patients than in normals suggesting that renal vasoconstriction may occur in ICU patients with normal renal function. Finally, the use of Duplex Doppler Ultrasound as a non-invasive method for the clarification of renal haemodynamics in humans it was clearly shown.

\section{SPACE 1366}

$X$-ray endovascular occlusion of the central adrenal veins - in treatment of patients of renin-dependent arterial hypertension with secondary aldosteronism

E. B. Mazo (Presenter), A. S. Akopyan, M. V. Koryakin, R. V. Anakhasyan; Moscow

The authors propose a new method of treatment of arterial hypertension by $\mathrm{X}$-ray endovasular occlusion of the central adrenal veins.

The use of the new method would help portalize adrenal venous blood circulation as well as to inactivate cathecholamines and aldosterone.

The proposed method has bean successfully used in 34 cases of essential arterial hypertension as well as in cases of arterial hypertension caused by chronic pyelonephritis and glomerulonephrities.

The results of the use of this method would make it possible to discuss at a new angle the treatment of patients of renindependent arterial hypertension complicated through secondary aldosteronism.

\section{SPACE 1367}

Color Doppler assisted duplex sonography in the stenosis of the carotid arteries

P. Milassin (Presenter), E. Nagy, Z. Morvay; Szeged

In 82 patients color Doppler assisted duplex sonography and angiography were performed. The grade of stenosis was divided into 6 groups. The same diagnosis was established with both methods in $80 \%$ of the cases. In most of the remaining cases with sonography a higher grade of stenosis could be observed than with angiography. Technical pitfalls in sonography of three cases caused significant differences in the evaluation of grading. Our results suggest that color Doppler duplex sonography should precede the application of other methods in the diagnosis of carotid stenosis.

\section{SPACE 1368}

Intra-arterial technique in advanced breast cancer

C. Sogo Manzano, B. Acea Nebril, R. Gayoso Garcia, A.Parjo Calvo, J. Mosquera Oses (Presenter); La Coruña

Breast cancer is one of the major problems affecting occidental women of today. In the U.S.A. every four minutes a breast tumor is diagnosed and every twelf a woman dies due to this cause. In Spain we will diagnose approximately 40,000 breast cancers in 1991 and 4,000 patients will die this year.

Our objective is to present a new technique, that following the studies started by I. Koyama in Japan improves the interval free of disease and the survival rate and also permits a less mutilant surgery.

We will show the supraselective intra-arterial chemoembolization technique in stage III cancers, that through the internal and external mammary arteries makes it easier for the medication to reach the tumor and later closing the vascularization.

Our results are backed by our experience in 17 cases in which we have obtained a survival rate of $55 \%$ at 5 years. This represents a clear advance due to the prognosis of the cases in which this technique were applied to.

\section{SPACE 1369}

Significance of magnetic resonance imaging (MRI) in patients with pulmonary hypertension

A. Neuhold (Presenter), H. Frank, M.Stiskal, S. Globits, D. Glogar, J. Miczoch; Vienna

Purpose: To determine the diagnostic impact of MRI in pulmonary hypertension (PH) 21 patients (pts.) were studied.

Methods: Double-angulated multislice-multiphase images (using a 0.5 Tesla magnet) were recorded using spinecho $\mathrm{Tl}$ and gradient echo sequences. MRI studies were analysed for right ventricular (RV) volumes, function, segmental wall motion, and diameters of pulmonary arteries and caval veins. These measurements were compared with pulmonary pressure measured within 48 hours of MRI. The underlying disease was in 3 pts. VSD, in 2 pts. ASD, 4 pts. suffered a drug induced pulmonary hypertension, 5 pts. had mitral valve disease, 2 pts. had multiple pulmonary embolism, and 5 pts. a dilated cardiomyopathy. In addition 10 healthy volunteers were studied.

Results: Pts. with PH showed increased RV-volume indices and abnormal septal motion irrespective of severity of PH. RV function was reduced in 3 pts., tricuspid regurgitation was demonstrated in all pts. There was good correlation between pulmonary mean pressure and diameter of inferior vena cava $(r=0.82)$, right ventricular EF and volumes showed no significant correlation. Conclusions: pts. with PH show a typical pattern of the right heart, although alterations do not correlate with the degree of $\mathrm{PH}$, with exception of inferior vena cava diameters.

\section{SPACE 1370}

Supraselective embolization in renal trauma: our experience Guillermo Parga (Presenter), Rafael Fernández, Roberto Villar, Ramón Garcia-Martin, Javier Abad, Enrique Garcia-Hidalgo; Madrid

We report 23 cases of renal trauma treated with supraselective embolization. In 7 cases the cause of the renal injury was iatrogenic due to aggressive procedures: biopsy, nephrostomy...

The embolization was made with two types of embolic materials: gelfoam and blood clot; and in a few cases with stainless-steel coils too. All the patients had resolution of their disease after the embolization. No complications appeared. We think that supraselective embolization is a good treatment in some cases of renal trauma.

\section{SPACE 1371}

Prosthetic aortic valve for percutaneous transcatheter insertion D. Pavcnik (Presenter), K. C. Wright, S. Wallace, C. Gianturco; Ljubljana, Houston, TX

Heart valve replacement began thirty-six years ago when Hufnagel surgically implanted the first valve prosthesis in a descending aorta. All of the prosthetic cardiac valves used clinically have been implanted surgically.

Our objective in this project is to construct and experimentally evaluate an alternative device which can be placed percutaneously, obviating the need for general anesthesia and open heart surgery. To date, eleven adult mongrel dogs have received our prosthetic valve. Results of these studies have led to several design modifications.

At present, prosthetic valve is a ball-in-cage design. The valve system can be collpased and pushed through an 11-French catheter system. Since the assembly is self-expanding, it opens as it exits the catheter. The final step is placement of the ball into the cage. Additional studies are now in progress to evaluate the current design with regard to thrombogenicity, hemodynamic function, durability and biocompatibility.

\section{SPACE 1372}

\section{Ultrasound gulded percutaneous placement of Hickman catheters} P. J. M. Post (Presenter), J. S. Laméris, H. M. Zonderland, H. E. Schütte; Rotterdam

Access to the subclavian vein for percutaneous placement of Hickman catheters is usually obtained by "blind" percutaneous puncture. Possible complications of this technique are pneumothorax, hemothorax, arterial punctures and nerve injury. We describe a new technique using ultrasound guided puncture of the target vein and fluoroscopic control of the guide-wire and the catheter position.

With ultrasound the subclavian vein and the surrounding anatomical structures, can be visualized, thereby minimizing the risk of the puncture related complications. Controlling the position of the guide-wire and catheter by fluoroscopy reduces the change of catheter malpositions.

Using this combined technique we performed 82 catheter placements. Long term administration of chemotherapy for hematological malignancies was the most frequent indication to place the Hickman catheter.

All punctures were successful and no puncture related complications occurred. We consider ultrasound guided percutaneous placements of Hickman catheters superior to other methods.

\section{SPACE 1373}

Comparative tests on thrombogenicity of some angiographic catheters H. Söder, R. Raininko (Presenter); Helsinki

We compared thrombogenicity of six angiographic catheters of four materials: nylon (Mallinkrodt, Surgimed), polyamide polymer (Meditech), polyethylene (Cook), and polyurethane (Cordis, Usci).

Each catheter was used in two pieces: one untreated and the other filled with heparinized saline. Catheters were filled with blood from a healthy donor and incubated at $37^{\circ}$ for 5 or 15 minutes. Then physiologic saline was injected through the catheters with a flow of $5 \mathrm{ml} / \mathrm{s}$ and clots were identified on a filter. EDTA was used to stop coagulation. The catheters were re-injected with a flow of $50 \mathrm{ml} / \mathrm{s}$ and clots were registered. 23 donors were used and 552 catheters tested.

All untreated catheters contained a clot at $15 \mathrm{~min}$ and $59 \%$ at $5 \mathrm{~min}$. Differences between the catheters were not significant $(p>0.5) .96 \%$ of the heparinized catheters contained a clot at $15 \mathrm{~min}$ and $24 \%$ at $5 \mathrm{~min}$. Heparini- 
zation reduced the number of the clot containing catheters highly significantly at $5 \min (\mathrm{p}<0.001)$ but differences between the catheters were small: the only significant difference was found between the catheters by Mallinkrodt and Usci (clots in $9 / 23$ and $2 / 23$, respectively, $p<0.02$ ). High pressure injection never revealed clots after a negative low pressure injection. This indicates that clots are loosely, if at all, attached on the catheter wall.

\section{SPACE 1374}

Assessment of early local thrombogenicity after percutaneous angioplasty with Tc-99m-HMPAO-platelets

J. A. Reekers (Presenter), M. R. Hardeman, M. M. C. van Buul, A. J. van der Kleij; Amsterdam

Platelets labelled with in-111-oxine require more than 24 hours after administration before a definitive scintigraphic assessment of thrombogenicity can be made. The aim of our study was to investigate whether platelets labelled with Tc-99m-HMPAO would allow evaluation of thrombogenicity shortly after intraluminal intervention. Autologous platelets, labelled with Tc-99m-HMPAO were re-injected an hour before angioplasty. Scintigrams were made before, as well as 1 and 4 hours after angioplasty. In 5 patients studies thusfar, accumulation of Tc-99m was found at all 6 sites of angioplasty one hour after intraluminal intervention. Scintigraphic images, performed 3 hours later, did not yield extra information. These results demonstrate that this technique is a sensitive and rapid method to assessing the thrombogenicity of (damaged) endothelium and has a practical avantage above currently used methods because the total procedure (blood withdrawal-angioplasty-scintigraphy) can be performed within 4 hours. The method cannot be used for platelet survival studies, but may be of use for evaluation of the effect of thrombocyte inhibiting medication.

\section{SPACE 1375}

Fusion of DSA-sequences and ECT-images to display morphological and functional myocardial information

N. Rilinger (Presenter), H. Niemann, G. H. Reil, A. Hewett, J.Schwanke, L. Köhler, J. Schwanke, P. Jensch; Oldenburg

It is our aim to support the clinical routine in the management of coronar heart disease by integrated diagnoses. For this purpose we pursue a fusion of coronary angigrams and ECT images, to integrate morphological and functional images.

Methods: Currently we are able to merge coronary trees, obtained from post-processed bi-plane angiogram sequences, with myocardial scintigrams after administration of thallium 201. This approach of fusion results in innovative multidimensional models whose constituents may be depicted in different colours, at arbitrary angles, and annotated with density information. A series of displays may also be viewed as an animated 3D-model showing the coronary tree and the ECT-data illustrating perfusion and function of the myocardium.

Results: To be able to perform integrated imaging repetitively we defined standards and processing facilities for each individual imaging system. Parts of the image data are prestructured, allowing specific items to restrict ROIs in an object-oriented and model-based way.

Conclusions: This paper demonstrates the normalization and matching processes and shows the advantage of information-integration modelling. The presented concept allows a combined diagnosis of morphological and functional data in the management of coronar heart disease.

\section{SPACE 1376}

A comparative study of the efficacy of lower-limb isotopic phlebography, Doppler-sonography and conventional phlebography in acute pulmonary embolism

A. Segarra (Presenter), M. Moreiras, J. M. Rius, R. Boyé, E. Grivé, M. Fraile; Barcelona

We instituted a prospective blinded study to assess the accuracy of DopplerSonography and isotopic phlebography in the detection of deep venous thrombosis (DVT) of the lower extremities, using contrast phlebography as the gold standard.

45 patients, between 22 and 78 years old, gammagraphically suspected of having acute pulmonary embolism were studied by contrast phlebography. 43 of these patients had Doppler-Sonography and 34 had Isotopic Phlebography. Contrast venography was positive in 29 patients for DVT. DopplerSonography was positive in 22 and normal in 21 patients (sensitivity was 1 , specificity was 0.72 ). Isotopic Venography was pathologic in 15 and normal in 19 patients (sensitivity was 0,74 , specificity was 0,93 ).

Contrast venography is the most accurate study in the detection of DVT of the lower extremity. Doppler-Sonography and Isotopic Venography because of their noninvasiveness and specificity could be used as prior filter to contrast venography.

\section{SPACE 1377}

Bronchial arteriography and therapeutic embolization in hemoptysis A. Segarra (Presenter), J.M. Rius, M. Moreiras, E. Grivé, L. Casas, C. Sánchez; Barcelona

A group of 63 patients ( 51 men and 12 women) between the ages of 21 and 79 years, with pulmonary hemorrhage, were evaluated by means of bronchial arteriography and treated with transcatheter embolization. Chest X-Ray film was obtained in all the patients been orientative in only 30 cases. Bronchoscopy was performed in 53 patients and could locate the bleeding site in only 38 . Arteriography was positive in 54 patients.

Angiographic signs include: hyperplasia of the bronchial arterial trunk and branches, extravasation of contrast medium, bronchopulmonary anastomoses and bronchial arterial aneurysms.

Results: Transcatheter embolization was performed in 48 cases. 41 of them stopped immediately. Recurrent bleeding 24 hours after the procedure was observed in 3, and only partial improvement was obtained in 4 . The treated patients were followed 6-30 months: Recurrent bleeding was observed in 5 patients. 3 of them underwent surgery, 1 was re-embolized and 1 died. Major complications in our series were: subintimal dissection (4), acute iliac thrombosis (1), segmentary mesenteric ischemia (1) and mediastinal hematoma (1). Conclusions: Transcatheter therapeutic embolization may be a very useful method for the control of hemoptysis in nonoperative candidates and for initial hemostasis before surgery in the operables ones.

\section{SPACE 1378}

Comparison of ${ }^{99 \mathrm{mTC}-M I B I}$ and ECG in exercise and rest conditions for the diagnostics of CAD

E. Sheiretova (Presenter), M. Garcheva, H. Hadjikostova, L. Terzieva, S. Kovacheva; Sofia

67 patients with CAD were examined with ${ }^{99 \mathrm{~m}} \mathrm{Tc}-\mathrm{MIBI}$ at rest and bicycle exercise. The diagnostic value of MIBI was compared with that of the classical ECG working test. In 22 patients with suspected CAD, MIBI and ECG test were negative in $77 \%$ and positive in $4.5 \%$. MIBI was positive in addition in $14 \%$; ECG test in $4.5 \%$. In 24 patients with stable or unstable angina pectoris both tests were negative in $38 \%$ and positive in $16 \%$. MIBI was positive in addition in $34 \%$; ECG test in $12 \%$.

For patients with previous myocardial infarction both methods were confirmative in $48 \%$ and without diagnostic value in $14 \%$ (cases with non-Q myocardial infarction). MIBI didn't visualize the scars in $33 \%$-cases with postero-inferior localisation. In $5 \%$ there was a difference between the localisation of MIBI-defect and the ECG-changes. Ischemic changes in this group were detected in $48 \%$ of the patients: in $24 \%$ by both methods, in $19 \%$ by MIBI and in $5 \%$ by ECG test.

Conclusions: In the visualization of scare the diagnostic value of MIBI is high, but not for the postero-inferior region. For ischemia detection MIBI is more successful than ECG working test.

\section{SPACE 1379}

Evaluation of cardiac function by serum human atrial natriuretic peptide Mitsuharu Shimizu (Presenter), Yanako Hirata, Shinobu Nakagawa, Michiko Agiki, Shinji Hashimura, Yoshio Hiraki; Okayama

Purpose: Serum human atrial natriuretic peptide (hANP) was measured in subjects consisting of 58 heart disease patients. A comparison was made of these results with cardiac function parameters determined by echocardiography, cardiocatheterization, cardiac blood pool scintigraphy. A study was then made regarding the evaluation of cardiac function by hANP.

Methods and Materials: Measurement of serum hANP was performed by radioimmunoassay. Testing was performed on 58 subjects consisting of 23 cases of ischemic heart disease, 30 cases of valvular disease and 5 cases other related disorders.

Results: In the elevated hANP group, left ventricular ejection fraction and cardia index were significantly lower in comparison to the normal group, and hANP reflected a decrease in left ventricular function. Also in the elevated hANP group, left atrial diameter was significantly increased in comparison to the normal group, and a significant positive correlation was observed between hANP and mean right atrial pressure. It was concluded that hANP become elevated accompanying extension of the atrial wall, and a parameter which reflects decreased left ventricular function.

\section{SPACE 1380}

The lelomyosarcoma of the vena cava inferior: a difficult diagnosis P. Vandermarcq (Presenter), J.B. Talon, A. Alluaume, J. Drouineau, O. Azais, A.S. Lagrange, C. Gasquet; Poitiers

a) The preoperative diagnosis of leiomyosarcoma of the vena cava inferior is very rarely made because of the scarcity of this affection. 
b) Two cases with suggestive preoperative modern imaging are reported.

c) Three radiologic investigations are useful:

- Both sonography and vena cava inferior angiography show either hyperechoic intraluminal polylobed lacuna located on one segment of the vena cava inferior without thrombosis or obstruction with collateral circulation and hyperechoic right paraaortic mass.

- CT demonstrates the extraluminal extension, the right lateroaortic location of the tumor and the heterogeneous soft tissue density where the vena cava inferior abuts. The association of an intraluminal lacuna and a right sided tumor seems to be very suggestive for the diagnosis.

These three radiological investigations are equally essential for the assessment of this tumor which has a bad prognosis and needs a delicate surgery.

\section{SPACE 1381}

Digital subtraction angiography (DSA) in cerebral and arterio-venous malformation

K. Velkova (Presenter), P. Stefanov, V. Chervenkov; Plovdiv

Presented is the experience yielded from 347 cerebral angiographic examinations. Among them were 54 cases (15\%) of cerebral aneurysms (CA) and 28 cases $(8 \%)$ of arterio-venous malformations (AVM). Analysed are both, the CA's and AVM's regarding position, size and status as well as the angiographic symptomatology, related to these factors. An attempt is also done to evaluate the diagnostic information of the intravenous and intraarterial DSA in cases of CA and AVM. The personal preference of the authors is to utilize the intraarterial DSA as primary preserved diagnostic test, when the place for the intravenous DSA is reserved in follow-up examination. Emphasized is the opportunity provided by DSA for analysing the morphology and hemodynamics of the CA-AVM using less contrast medium and reducing the radiation hazard.

\section{SPACE 1382} Thermal distribution of sapphire probe: influence of flow rate of $\mathrm{CO}_{2}$ gas
and saline perfusion

Xiaoming Yang (Presenter), Hannu Manninen, Hongxiu Ji, Seppo Soimakallio; Kuopio

Purpose: To evaluate thermal distribution characteristics of sapphire probe. Methods: Together 162 temperature measurements on a $2.2 \mathrm{~mm}$ sapphire probe surface were obtained in a circulation model within $37^{\circ} \mathrm{C}$ flowing whole blood. A thermocouple wire was directly contacted onto the sapphire probe at: 1. metal connector, 2. lateral side and 3. top of sapphire crystal. During lasing with Nd-YAG laser, carbon dioxide $\left(\mathrm{CO}_{2}\right)$ gas or saline was infused through the sapphire probe at different flow rates.

Results: Mean peak temperatures at all three sites increased with increasing flow rates of $\mathrm{CO}_{2}$ gas and decreased with increasing flow rates of saline perfusion. Mean temperature rise at the lateral side of the sapphire crystal was statistically lower $(\mathrm{p}<0.05)$ than that at the sapphire crystal top, but higher than that at the metal connector. Moreover, the mean temperature rise at all three sites was statistically higher $(\mathrm{p}<0.05)$ with $\mathrm{CO}_{2}$ gas than with saline perfusion.

Conclusions: Present study shows the experimental basis: 1) there is direct thermal conduction from the lateral side of the sapphire crystal. 2) $\mathrm{CO}_{2}$ gas perfusion offers more favorable mode of action for the thermal efficiency of the sapphire probe than saline perfusion.

\section{SE Mammography (03) \\ SPACE 1383}

Results of the revision of the first 20,000 women participants in an early detection breast cancer program in Navarra, Spain

Luis Apesteguia Ciriza (Presenter), Alberto Murillo Espinal, Angel Del Moral Aldaz, Nieves Ascunce Elizaga; Pamplona

Purpose: Detection of breast cancer in its early stages in women residents in Navarra betwen 45 and 65 years old.

Methods and Materials: Screening technique: a mammograph (OML projection). Initially 5,000 women were involved in a double blind reading until satisfactory agreement rates were reached. Later, only random samples of the women examined $(15 \%)$ were carried out.

In necessary cases new mammograph projections, physical examinations, FNA-citologies are carried out.

Citologies for estereotaxia and biopsies with and without radiological localization for a definite diagnosis and treatment, will be carried out in 2 tertiary-level hospitals with the participation of radiologists from the detection unit.

Results: To date have been examined 10,436 women. New mammograph projections were necessary in $15 \%$ of the cases and $3 \%$ needed an echograph.
$2 \%$ of the women were sent to the hospital unit for citology and/or biopsy.

To date 58 cancers have been detected, giving a detection rate of about 5 per 1,000 of a predictive positive value of $34 \%$.

These results will be presented along with stages, treatments applied and percentages of the application of different techniques for women examined, up to 31 July $1991(20,000)$.

\section{SPACE 1384}

The early detection breast cancer program in Navarra, Spain, general organisation, participation data

Nieves Ascunce Elizaga (Presenter), Angeli Del Moral Aldaz, Luis Apesteguía Ciriza, Alberto Murillo Espinal; Pamplona

Purpose: Reduction of breast cancer mortality, increase survival rate, improve quality of life.

Methods and Materials: Carry out mammographs (OML) every 2 years on all women resident in Navarra $(60,000)$ between 45 and 65 through personal citations (census based). Test are free.

In all cases an epidemiological questionnaire will filled out with hormonal, gynaecological and mammary data.

A permanent urban unit and rural mobile unit have been set up, equipped with mammographs and processors. Personnel in each unit is formed by sanitary auxiliaries and radiology technicians. The general organisation of the program has been entrusted to the manager, radiologists and secretaries of the central coordination unit.

2 hospitals have been entrusted with the diagnostic and treatment process, according to previously agreed protocols.

Participation will be obtained through the collaboration of primary health teams using too press, radio, TV, posters, pamphlets, and conferences. This program is included in the European network supported by the EC (Europe against cancer), being piloted in Navarra for Spain.

Results: The general coordination program scheme, along with strategies to attract women, the organisation of units etc. will be presented.

To date, 10,436 women have been examined $(82.3 \%$ of those invited to participate), and 58 cancers confirmed.

\section{SPACE 1385}

Correlations of CA-15-3 radioimmunoassay values in patients with breast cancer with radiologic and clinical evaluations

Kirkman G. Baxter (Presenter), James Bergh, David F. Preston, Ralph G. Robinson, Arch W. Templeton; Kansas City, KS

Purpose: The CA-15-3 assay utilizes monoclonal antibodies that react with a circulating antigen expressed by human breast carcinoma cells.

Methods and Materials: We examined the relationship of CA-15-3 radioimmunoassay values to the scintigraphic, radiographic, and clinical evaluations of patients with metastatic breast carcinoma. The CA-15-3 values were correlated with imaging impressions of improvement, worsening, or no change. Patients who had insufficient radiographic evaluation were rated by final clinical impression of the current status of their disease.

Results: There is a positive correlation of CA-15-3 values with the level of soft-tissue metastatic breast cancer involvement. This new assay provides useful clinical information regarding the status of metastatic breast carcinoma.

\section{SPACE 1386}

\section{Phosphor plate mammography}

C. H. Joseph Chang (Presenter), Norman L. Martin, Larry T. Cook, Michael F. Insana, Samuel J. Dwyer III, Arch W. Templeton; Kansas City, KS

Purpose: A photostimulable phosphor imaging plate system has been used for over one year for mammography examinations.

Methods and Materials: Conventional screen-film and phosphor plate mammograms were obtained. Special high resolution cassettes and imaging plates were used. A 50 percent exposure reduction was employed for the imaging plate examination. Digital mammogram images were archived on an optical disc. A comparison between conventional and reduced exposure phosphor plate mammograms has been completed for patients with masses, micro- and macrocalcifications, and dense fibrocystic changes. This study shows equivalent results.

Results: The inter relationship of the linear wide dynamic range and improved imaging efficiency of phosphor plates is discussed. 


\section{SPACE 1387}

\section{Sensitivity and speciflcity of ultrasound in palpable breast masses} J. Daneš (Presenter), L. Vyhnánek, E. Gutwirthová; Praha

Methods: The purpose of the study is to estimate the reliability of the ultrasound investigations in comparison with the mammography in the diagnostics of the pathological conditions of the breast.

Methods and Materials: The study is based on experiences with the ultrasound examinations of the breast in more than 3000 women. From this group of patients, 508 selected women with the palpable lesion of the breast were simultaneously examined by mammography and ultrasonography. All of the findings were verified.

Results: In the estimation of the carcinoma, the sensitivity of the US was higher $(98 \%)$ than of the mammography $(78 \%)$. On the contrary, the specificity was a little lower $(94 / 96 \%)$. The ultrasonography proved to be especially valuable in the women with dysplasia of the mammary gland, in which the sensitivity of the mammography was very low (58\%). Excellent results were achieved by the combination of both of the methods. Ultrasonography was highly reliable in the diagnosis of the cysts, including galactoceles and inflammatory lesions, too.

\section{SPACE 1388}

\section{Sonographic features of cystosarcoma phylloides}

T. H. Dao (Presenter), T. Nguyen-Tan, L. Ollivier; Paris

Purpose: To assess the ability of sonography to identify cystosarcoma phylloides when $\mathrm{X}$-ray mammography reveal only benign-appearing mass compatible with other diagnosis.

Methods and Materials: Sonographic features of 9 cases of proven cystosarcoma phylloides are reviewed.

Results: The ultrasound findings included hypoechoic multilobulated lesions with sharp demarcations, posterior enhancement. These lesions are predominantly solid with fluid-filled space surrounding nodular lesions, usually described as a "cleft" pattern. Association of this aspect of "bunch of grape" with this cleft pattern is, in our experience strongly suggestive of cystosarcoma phylloides. Computed tomographic and histologic correlations are shown. Conclusions: When a "bunch of grape" pattern is found on sonography, the diagnosis of cystosarcoma phylloides must be considered for adequate treatment, until sarcomas are indistinguishable from benign lesions.

\section{SPACE 1389}

Mammography with dual screen and dual emulsion registration systems C. Dimaggio (Presenter), P.Rossi, S. Galassini, M. Lagrassa, L. Bidoli, L. Pescarini; G. Moschini, S. Recanatini, M. Pavanello; Padua

Purpose: Is it acceptable to use the grid systematically in mammography? With grid, mammographic images are qualitatively better, anyway the advantages in terms of diagnostic contribution have not yet been quantified with certainty and the dose is greater.

The aim of this study is to value the possibility of employing more sensitive systems in such a way as to can obtain the advantages by using the grid without increasing the dose.

Methods: A conventional mono-emulsion system (KODAK MIN-R/ORTHO-M) used without grid was compared with a new system using dual reinforced screen and dual emulsion film (FUJI HR-FAST/HR-MH) used with the interposition of the mobile grid.

Qualitative valutations and quantitative ones (H\&D curve, "Wiener spectrum", MTF) have been done.

Results: The dual-emulsion films appeared to be better in sensitivity and contrast and slightly noisier. The resolution was superimposable.

Conclusions: The results suggest that if the grid is used systematically it is better to use more sensitive systems to reduce the dosimetric problem. The dual-emulsion system used in this study is suitable.

As a last consideration, the use of a dual-emulsion film minimizes the problems deriving from bad treatment and makes it possible to utilize quicker treatment times.

\section{SPACE 1390}

Nonpalpable breast lesions: double localization with wire and dye track P. Cosmacini, V. Sacchini, C. Ferranti (Presenter), L. Aiani, G. Piragine, S. Bergonzi, G. Coopmans de Yoldi; Milano

A method of double localization, in order to make easier the remotion of nonpalpable breast lesions, has been performed at the National Cancer Institute of Milan.

Between August and October 1990, 10 consecutive patients underwent double localization and then surgery. The first localization is performed the day before surgery, placing by means of mammography a self-retaining anchor wire needle near the lesion; the second localization is performed immediately before surgery, injecting a Methylen Blue solution through a lateral-holed needle. This needle is introduced cannulating the previously placed wire. The Methylen Blue solution spreads through the lateral holes situated in the last $\mathrm{cm}$ near the point of the needle, making a dye track that leads to the lesion.

In our 10 cases the dye track was always well visible, with a width until $1.2 \mathrm{~cm}$ around the wire. This method allows the surgeon who follows the dye track to easily recognize the lesion and to plan an adequate and radical excision, avoiding to cut the wire.

\section{SPACE 1391}

Mammographic study in male-to-female transsexual in hormonal therapy

Maria Grazia Genovese (Presenter), Renato Bocchini, GianPaolo Cavallotti, Riccardo Violante; Torino

Often the male transsexual takes hormonal drugs to obtain a development of mammary gland and the disappearance of hair from facial skin.

The authors present radiological aspects of breasts in 20 patients (age between 20 and 46 years) before and after therapy of 2 years at least.

Nobody transsexual had mammary lesion before hormonal therapy.

After therapy we observe 6 normal and 14 pathologic radiographic reports (7 adenofibrosis, 5 fibrocystic disease and 2 patients with dystrophic calcifications) without breast cancer.

We relate work-up in table.

\section{SPACE 1392}

Primary lymphoma of the breast, report of 4 cases

A. Gomez Gomez (Presenter), E. Castañer, V. Villalba, A. Zidan, C. Traid, Y. Pallardo, P. Alvarado; Barcelona

Purpose: Primary non Hodgkin lymphoma of the breast is a rare entity, and few cases have been reported in the radiological literature.

Methods and Materials: We present 4 patients with primary breast non Hodgkin lymphoma.

Results: They presented with palpable masses associated with skin edema and axillary lymph nodes in one case. Plain mammography showed ill defined irregular masses, considered as malignant, in two patients, and well defined masses, considered as indeterminated, in the other two. One patient presented diffuse breast edema, and skin thickening. No microcalcifications were demonstrated. Pneumooncography (air injection into the lesion) showed cryteria of malignancy in the indeterminated masses. The preoperative citology was not diagnostic. No case diagnosed preoperatively as lymphoma. The peroperative biopsy diagnosis excluded epitelial neoplasm but no definitive diagnosis could be made.

Primary non Hodgkin lymphoma of the breast can be differentiate preoperatively from other malignant lesions, only definitive pathologic study can made the diagnosis.

\section{SPACE 1393}

\section{Breast tuberculosis}

V. Villalba Nuño, A. Gomez Gomez (Presenter), M. Velasco; Barcelona

Affectation of mammary glands by Tuberculosis (Tbc.) is not frequent. Out of 86.500 explorations carried out within the last 22 years, 5 cases have been located. In one of them ( $1 \mathrm{c}$.), association with carcinoma was observed. Presentation radiologic standards are checked, and diagnostic methodology is expounded.

Results: 1. Affectation non-frequent $(0,05 \%)$. 2. Presentation radiologic standards in common with malignant lesions. 3. Diagnosis guided by the presentation of Tuberculosis in other organs. 4. Non-conclusive citology. 5. Histological and microbiological diagnosis. Unless bacillus is isolated, diagnosis labelled as Granulomatous Caseose Mastitis.

\section{SPACE 1394}

Value of pneumooncography in the diagnosis of medullary carcinoma and colloid carcinoma

A. Gomez Gomez (Presenter), A. Zidan, E. Castañer, Y. Pallardo, C. Traid; Barcelona

Purpose: Colloid carcinoma (mucinous) (CC), and medullary carcinoma (MC) of the breast usually present as well circumscribed lesions that are difficult to differentiate from benign lesions. We described the value of pneumooncography (air injection into the lesion) in the preoperative diagnosis of these neoplasms.

Methods and Materials: We present $22 \mathrm{MC}$ and $24 \mathrm{CC}$ which represent respectively $0,5 \%$ and $0,6 \%$ of all breast cancers diagnosed in the last 17 years in our center. The mean age (range) was 56,5 (21-79) for MC and 52,5 (45-83) for CC. The mean duration of symptoms was 3,5 months for MC and 16 months for CC. 
Results: 10 of the MC (45\%), and 15 of the CC $(62,5 \%)$ presented as a well circumscribed mass and underwent pneumooncography. This showed criteria of malignancy and consisted in: 1. Absence of capsule in all of them. 2. Vaquolar pattern in $7(70 \%)$ of $\mathrm{MC}$, and in $8(53 \%)$ of CC. 3. Demonstration of irregular contours in all MC and $11(73 \%)$ CC. 4 . Absence of malignant ductal pattern, which is diagnostic of ductal carcinoma, in all of them. Pneumooncography is a valuable aid in the diagnosis of MC and CC.

\section{SPACE 1395}

\section{Nonpalpable breast lesions: mammographic and localization procedure imaging, review of 90 cases}

M. Iribar (Presenter), A. Vilarrasa, S. Borruel, C. Lopez, C. Miro; Madrid

Radiographically guided localization of nonpalpable breast lesions before biopsy avoids unnecessary excessive removal of breast tissue. Marking of these lesions for biopsy has become a routine procedure in patients in whom mammography has suggested malignancy.

We present 90 cases of occult breast lesions detected by mammography in which biopsies were performed from 1984 to 1990 . The patients ranged in age from 30 to 80 years, with a mean of 44 years. The radiographic lesion size ranged from 2 to $40 \mathrm{~mm}$ (mean $13 \mathrm{~mm}$ ) and 15 cases had previous diagnosis of malignancy in the contralateral breast. Suspicion of malignancy was based on the presence of the following radiological patterns (given together with their Positive Predictive Value PPV): $1 /$ microcalcification alone (PPV 0.40), 2/stellate opacities (PPV 0.64), 3/asymmetric densities (PPV 0.16), 4/regular border densities (PPV 0.27), and 5/microcalcifications associated with 2,3 or 4 (global PPV 0.46). Neither the presence of microcalcifications in a pattern of stellate opacities nor its prensence in a pattern of regular border densities seems to yield a higher suspicion index than both patterns alone (PPV 0.62 vs 0.64 and PPV 0.20 vs 0.27 respectively). On the other hand, the higher PPV (0.50) of asymmetric densities with microcalcifications compared to that of asymmetric densities alone $(0.20)$ should be taken with caution due to the small number of cases. The average detection rate of preclinical cancer was $2.10 \%$ and the ratio of benign to malignant biopsy findings was $1.6: 1$ for the whole series.

Examples of mammographies will be shown, as well as tables with the results according to age group, radiological pattern and histological diagnosis.

\section{SPACE 1396}

\section{Inflammatory carcinoma of the breast: clinical and roentgenologic} findings

U. Keske (Presenter), M. Langer, P. Uhrmeister, R. Felix; Berlin

Purpose: Clinical and roentgen findings of inflammatory carcinoma of the breast (IC) were evaluated retrospectively.

Patients and methods: A total of 39 patients was examined. Histologic confirmation of the diagnosis was present in all cases ( 26 primary/13 secondary IC). Results: Mean age was 55.8 years and slightly higher for secondary than for primary IC (61.5/54.6 years). Patients presented with a firm induration of the entire breast $(83 \%)$ and palpable axillary lymph nodes $(69 \%)$. Redness of the skin $(57 \%)$ or a palpable tumor $(46 \%)$ were other frequent criteria. The roentgen findings were dominated by an overall increased tissue density of the breast $(100 \%)$ and skin-thickening $(97 \%)$, a localized tumor could only be seen in $27 \%$. Means survival was poor ( 21 months) and worse for secondary than for primary IC (14/28 months).

Conclusions: Whenever the clinical and radiologic criterias mentioned above are present, further evaluation for IC should be undertaken (cytology or skin biopsy). The latter proved to be very helpful in the diagnosis of lymphangiosis carcinomatosa.

\section{SPACE 1397}

Comparison of breast structure and findings by $x$-ray mammography and ultrasound

H. R. Laine (Presenter), J. Rainio, H. Arko; Helsinki

Even nowadays, X-ray mammography (XRM) cannot always reveal small breast cancers in breasts rich in dense normal glandular and/or mastopathic tissue. Ultrasound (US) is very sensitive for soft tissue characterization.

In this study we tried to evaluate the quality of the methods for our routine breast diagnoses in our hospital. This material has been collected during a year 1988-89. All XRMs and US examinations and fine needle aspiration biopsies (FNAB) of breasts performed to these patients, were collected. 489 female patients underwent XRM and 293 patients of these underwent also US examination and 88 patients FNAB, too. All cases, found cytological diagnoses PAPA Gr III-V were operated.

Results indicated, that $25 \%$ of malignant nodules would have been missed, if only XRM would be performed. The results also revealed, that the smaller nodule and/or denser breast tissue, the better visualization will be reached by US compared to XRM. This study also indicated, that only $20 \%$ of breasts, found malignant tumour, had calcifications of malignant type, whereas in cases, whose breasts contained malignant type microcalcifications in XRM, the malignancy was found in $50 \%$. We did not found any calcifications in quarter of the cases, found malignant tumour histologically. In conclusion we can present, that soft tissue breast examination by US must be performed always, especially, when dense normal glandular and/or mastopathic tissue is found in one or more quarters of a breast. That means, that number of unnecessary breast operations will be reduced and more sparing breast operations can be performed.

\section{SPACE 1398}

Double-contrast ductography: diagnostic evaluation of the ductal system

Jose M. Llerena (Presenter), Juan D. Berna, Vicente Garcia-Medina, Christopher Kuni; Minneapolis, MN

Purpose: To evaluate the role of double-contrast ductography or pneumoductography in the diagnostic evaluation of small intraductal lesions that can be obscured by contrast material used in the routine single-contrast ductography Methods and Materials: After routine single-contrast ductography and evacuation of the contrast material, we inject 0.5 to $2 \mathrm{ml}$ of air using a small $2 \mathrm{ml}$ syringe that is connected to a catheter inserted in the duct. Routine mammographic examination is performed prior to removal of the syringe and catheter in order to avoid air reflux.

Results: We used double-contrast ductography in 25 patients with a mean age of 46.8 years. Pneumoductography showed two $3 \mathrm{~mm}$ elevated lesions that were not seen during routine single-contrast ductography. Intraductal papilloma was diagnosed in both cases by histologic examination. Also our doublecontrast technique allowed us to differentiate intraductal lesions from artifacts seen during routine single-contrast. All the patients tolerated well the procedure and we did not find any complication.

We conclude that double-contrast ductography or pneumoductography is a complimentary technique to routine single-contrast ductography in the diagnostic evaluation of small lesions in the main duct. Pneumoductography allowed us a detailed view of the main duct walls and helped in differentiating true intraductal lesions from artifactual filling defects observed during routine ductography.

\section{SPACE 1399}

Mammographic changes after lumpectomy: review of 29 cases C. Lopez (Presenter), S. Borruel, A. Vilarrasa, M. Iribar, R. Calero, C. Miro; Madrid

Since more than fiveteen years there has been a trend toward conservative surgery in the treatment of operable breast malignancy. Surgical procedures ranging from radical mastectomy to lumpectomy with or without radiation. When lumpectomy is achieved a wide variety of postoperative mammographic abnormalities may be found: skin changes parenchymal scar, fat necrosis, asymmetric glandular tissue defect and local recurrence imaging may be stood out.

We searched 29 cases of breast carcinoma in last three years in whom lumpectomy was achieved in our institution. We show mammographic imaging of those postsurgical abnormalities and radiologic evolution. We emphasize differential criteria which can help distinguish benign scarring from malignant local recurrence.

\section{SPACE 1400}

Association of breast cancer steroid receptors with mammographic parenchymal pattern and age

D. Mileusnić (Presenter), M. Dičić, S. Kurbel, B. Radosavljević, O. Rubin, Z. Filaković; Osijek

Study included 105 unselected breast cancer female patients diagnosed and treated at the Osijek General Hospital, Yugoslavia. All patients were over 30 The procedure included clinical examination, mammography, surgery with pathohistology and steroid receptor levels.

The aim was to assess the reliability of parenchymal patterns in predicting the steroid receptor status and to correlate steroid receptor levels and mammographic patterns with age and menopausal status of the patients.

No significant associations were observed between the steroid receptor content in the primary tumor and mammographic parenchymal patterns, suggesting that it is not possible to predict hormonal dependency from the mammographic pattern.

P2 and Dy pattern were more frequent among premenopausal and patients under the ago of 50. (P2 44.2\%, Dy 32.6\%). Lower shares were observed in the group over 50 (P2 37.1\%, Dy $22.6 \%$ ). Observation was not significant (t-values 0.37 for $\mathrm{P} 2$ and 1.12 for Dy: $p>0.05$ ).

Estrogen receptors correlated strongly with age (maximum at the age of 60 ; $\mathrm{N}=77 ; \mathrm{R}=0.37 ; \mathrm{p}<0.01$ ). Progesterone receptors showed no significant correlation with age of patients but correlated with menopausal duration (maximum 20 years after menopause, $\mathrm{N}=40 ; \mathrm{R}=0.39 ; \mathrm{p}<0.05$ ). 
Physical examination, mammography and ultrasound in the detection of axillary lymph node metastases in breast cancer

J. Morgado Ferreira (Presenter), B. Assis, M. Flores, C. Cunha; Lisboa

A prospective blind study was carried out with the objective of assessing the value of physical examination, mammography and ultrasound in the detection of axillary lymph nodes metastases in patients with breast cancer.

We studied 84 female patients admitted consecutively at a surgical department of an oncological hospital with mammographic suspicious lesions. All patients were subject to a standard physical examination, axillary view and ultrasound performed by independent physicians.

$31.5 \%$ patients had a histological diagnosis of benignity. Of the remainder, $82 \%$ were subject to an axillary dissection and there were $40 \%$ of metastases in axillary lymph nodes.

Physical examination presented the higher specificity $(92.4 \%)$, but a very low sensitivity (45\%). Ultrasound had the higher sensitivity, $80 \%$, with $88.7 \%$ specificity.

There were no false positives in patients with benign disease. However, all methods failed to detect lesions smaller than $5 \mathrm{~mm}$.

\section{SPACE 1402}

A spectrum of unusual circumscribed diseases of the breast Joaquin J. Mosquera (Presenter), Esther Rodriguez, Alejandro Maté, José M. Castro, Luisa Arrojo; La Coruña

We report the mammographic and ultrasound findings in 12 patients with unusual circumscribed lesions of the breast.

During the last five years we have collected a spectrum of unusual malignant and benign circumscribed lesions studied with mammography and ultrasound. Final pathological diagnosis included: Giant Lipoma, Fibroadenolipoma, Papillary Carcinoma, intracystic Carcinoma, Adenoid Cystic Carcinoma, Primary Osteogenic Sarcoma, Primary Lymphoma of the breast, Metastasic Melanoma, Phylloides Tumor.

In Oil Cyst, Hematoma in male and abscess the diagnosis was made by clinical history, mammography and evolution of the lesions. Besides it the abscess was punctured and cultured.

\section{SPACE 1403}

Discomfort and pain in breast due to foreign bodies

V. Rathaus (Presenter), M. Shapira; Kfar Saba

5 cases of various radiopaque foreign bodies in the breast tissue were found in mammography. All women presented with discomfort and pain. In two of them a mass was also palpable.

In three of the patients unreported catheter was found to be for therapeutic purposes: no additional breast pathology was found. In the other two cases remains of previous surgical procedures causing the palpable masses were seen. These unexpected mammographic findings explained the patient's complaints and in fact mammography was the only study required to evaluate these women.

\section{SPACE 1404}

Duplex-Doppler sonography in the evaluation of breast masses R. Rodríguez (Presenter), R. Cortina, B. Vargas, N. F. Marcos, N. Alegre, V. Guadaño, M. J. Juanco, J.M. Fernández; Madrid

Purpose: Breast sonography can be used to differentiate cystic from solid masses, and to characterize masses as an adjunct to mammography. The combination of mammography and sonography can characterize with liability a high percentage of breast disorders. However in some cases the differentiation between benign and malignant breast conditions is difficult. The purpose of our work is to evaluate the role of duplex-Doppler sonography in the evaluation of breast masses.

Methods and Materials: All the women with solid breast masses assisted in our Service over a 9-month period, were included in the study.

A real-time sonography unit with Dopper capabilities was used for the breast examination. A $7.5 \mathrm{MHz}$ transducer ( $5 \mathrm{MHz}$ for Dopper analysis) was employed. The range gate sampling was $2-4 \mathrm{~mm}$. The lowest available filter of the Doppler unit $(100 \mathrm{~Hz})$ was selected. The scale of the velocity range of the spectral display was set at $0.25 \mathrm{~m} / \mathrm{sec}$.

Fine-needle aspiration-biopsy was performed on all patients immediately after sonography.

Results: In more than $50 \%$ of the patients with breast cancer there was positive tumor flow signals. No patients with benign breast conditions showed positive Doppler signal.

Therefore, duplex-Doppler sonography can play an useful role, as an adjunct to mammography and sonography, in the evaluation of breast masses.
Uitrasonography v.s. mammography in diffuse benign breast diseases Elena Rabanal Bodelon, Joan Salvia Farre, Roser Garcia Abella, Jaume Planas Roquerols, Ramon Rosell Mir (Presenter); Barcelona

We studied 250 women between 30 and 70 years, diagnosed radiographically as mammary dysplasia, as stablished by the following parameters:

A) Evenly dense breast: Predominantly fibrous disease

B) Breasts with multiple nodular images with more than $3 \mathrm{~mm}$ of $\varnothing$ : Fibrocystic disease

c) Nodular images with less than $3 \mathrm{~mm} \varnothing$ : Fibrocystic disease of ductal or lobular hyperplasia.

We have tried to establish a correlation between the images obtained by mammography and those obtained by sonography.

We have observed that the presence of nodules in the mammography, which we diagnosed as being small cysts or fibroadenomas, do not have sonographic reciprocity in certain cases.

Moreover dense breasts, that have evenly fibrous aspect may have cysts of up to several $\mathrm{mm}$ of $\varnothing$.

We believe that sonography confirms the need of a new classification of breast fibrocystic diseases, the review of the concept of mastopathy and the normality, of many explorations diagnosed radiologically as diffuse intraglandular disease.

The use of sonography and mammography together allows to establish a more precise diagnosis of benign breast diseases, and helps to confirm the presence or absence of nodules in dense breasts without palpable masses.

\section{SPACE 1406}

Breast cancer detection program in hospital workers

R. Salvador (Presenter), M. Campins, M. Olona, M. Martín, M. Salvador; Barcelona

We present a preliminary report on a breast cancer detection program in hospital workers, which was begun in January 1989 , and it was controlled and carried out by the Radiodology Department and the Preventive Medicine Department in the General Hospital Vall d'Hebron of Barcelona. It was a part of the main general reviews in the feminine workers of the hospital.

The program included the female hospital workers older than 45 and consisted in a physical examination and a double view mammogram. The patients that accomplished the revision were 336 (37.7\% of the 892 who were convocated). An elevated incidence of carcinoma was confirmed, 4 cases representing a $1.2 \%$. It probably represents the high prevalence of the disease, a fact reported by many authors in their first examination.

\section{SPACE 1407}

Hardness and softness, an objective and very valuable sign in breast ultrasonography

R. Salvador (Presenter), M. Salvador, M. Olona, J.Andreu, M. Martínez; Barcelona

We made an ultrasonographic study in 169 breast lesions included under two conditions: 1. Either clinical or radiological breast lump, and 2. Tumor bigger than $5 \mathrm{~mm}$. All the lesions were studied by ultrasound (US) and by a two-view mammography.

A new US maneuver is described and evaluated: The a. p. axis was measured and how it shortened under US probe-compression, classifying the lesions as probably malignant and definitively benign according to this sign alone. Three different patterns were observed:

1. - Malignant pattern. Those tumors with an upper compression limit of $1 \mathrm{~mm}$. All the malignant tumors were included in this pattern (sensitivity $=100 \%$, sepcificity $>60 \%$ ).

2. - Slight compression benign pattern. The border compression limit was stablished between 1 and $3.4 \mathrm{~mm}$. All of them were benign conditions: Fibroadenomas, Small cysts, epitheliosis, etc.

3. - Soft benign pattern. The lower compression limit was $3.4 \mathrm{~mm}$. Here were included only two different benign processes. Fatty tumors (lipomas, hamartomas), and the biggest cysts (more than $2 \mathrm{~cm}$ ). Not any malignant lesion was observed to diminish far more than $1 \mathrm{~mm}$.

\section{SPACE 1408}

Functional dimensions in early diagnostic of breast cancer U. M. Schuchard (Presenter), R. R. Haas, M. Peter, M. Mahlberger; Gießen

Purpose: By modifying the traditional technique of positioning and recording, we can approach better identification of clinically occult mammary carcinomas. Such advantages result by expanding the field of radiological symptoms

Methods: For additional observation of the elastic behavior of the mammary structures we proceed mammography with a simultane compression and 
traction of the female breast.

Results: Based on generally principles of elasto-mechanics an infiltrating mammary carcinoma can be recognized by a concentration of structures resulting from the concentrated tension and elongation. This finding can be demonstrated arithmetically and by stress analysis.

Further advantages of this Mammo-Elastogram are as follows: 1. Better view of the retromamillary space. 2 . Inclusion of the tissue which were contiguous to the pectoral wall into the $x$-ray beam. 3 . Control of the mobility of suspect mammary inclusions regarding the compression of peritumoral structures. 4 . Enlargement of the radiological projection of organs and thus a better view of detail. 5. Always the nipple marginally on the picture, giving a clear point of reference as to the localisation of the tumor.

Conclusions: Analogous with the retraction phenomenon which we have clinically in cases of breast carcinomas and proved by stress analysis, these tension and elongation concentrations which can be visualised radiologically and which have been described above, turn out to be most reliable radiological signs of malignity.

\section{SPACE 1409}

Malignant calcifications in mammographic appearance of invasive papillary breast carcinoma: report of two cases

Renato Silva (Presenter), Francesco Ferrozzi, Pietro Quaretti, Armando Rossi; Piacenza

The invasive papillary carcinoma is an uncommon neoplasm of the breast. The radiologic features were described recently (1). Our contribution is concerning two cases both occurred in the elderly (above $80 \mathrm{y}$.). Mammographic pattern was characterized by a cluster of round or oval, smoothly circumscribed areas of density in one quadrant of a breast. In both patients, calcifications were found (finding not previously reported). Sonography revealed hypoechoic solid, smooth-walled abnormalities. In agreement with others (1), we believe that the diagnostic imaging of the breast may be suggestive for invasive papillary carcinoma. It is noteworthy that the presence of malignant calcifications cannot rule out the diagnosis of papillary breast carcinoma when the well-known pattern is shown at mammography.

(1) Schneider J. A. "Invasive papillary breast carcinoma: Mammographic and sonographic appearance". Radiology 1989; $171: 377-379$.

\section{SPACE 1410}

Mammographic screening: what kind of future?

Cesare Tetti (Presenter), Maria Cristina Martina, Aurelio Tetti; Torino

The mammographic check-up is developing more and more among people and it involves asymptomatic women, for whom the age represents an indication to the test. Mass media have certainly influenced this line of conduct, so that the number of patients now turning to the clinical mammography is getting higher and higher.

The frequency of check-up shall depend on the "kind" of breast, i.e. its clinicalradiological characteristics, together with the careful evaluation of the risk factors.

We have considered 20.000 women who underwent mammography; the under 35 have been excluded from this study, since this age class is not covered by the records. Normal cases were $17.100(85.6 \%)$, neoplasias $720(3.7 \%)$, while in 2.150 cases (10.7\%) mammography gave suspicion data (Class R3) and required percutaneous or surgical bioptic checks. The diagnostic accuracy of the enquiries by image on the breast shall have the false positive as its term of comparison, which, more than the false negative, represents the real "mistake" in Senology.

\section{SPACE 1411}

Mammographic, sonographic and Color Doppler findings in patients with conservative surgery and irradiation (QU.A.RT.) of breast carcinoma

C. Vanoli, M. Carlotto, R. Campi, L. Tufarulo (Presenter); Como

Purpose: The normal appearance of irradiated and operated breast is presented in comparison with the abnormal findings of recurrent cancer. Methods and Materials: 150 patients have been examinated short time later surgery with clinical palpation, mammography and sonography.

40 patients presented suspicious findings for recurrent breast carcinoma. Mammography roused suspicion when there were microcalcifications, thickenings, distorsion of the normal breast structure. Sonography roused suspicion when there were solid hypoechoic nodes, a non-homogeneous structure of the breast, irregular outline of breast, cysticlike nodes. Color doppler was not useful. All $\mathbf{4 0}$ patients underwent thin needle biopsy.

Results: in 40 patients a recurrent cancer was suspected, but only in 15 patients we found a recurrency.

That is to say a specificity of $37,5 \%$ with the utilization of both mammography and sonography.

\section{SPACE 1412}

\section{The male breast}

M. Velasco (Presenter), A. Gómez, T. Pujol, M. Solé, J. Pomés; Barcelona

One hundred and sixty mammographic studies of male breast performed during the last four years were reviewed. All patients presented clinical breast induration. The youngest was a 12 year old and the oldest a 85 year old. The radiological findings were as follows: 137 cases af gynecomastia, three abscesses, three inflammatory mastitis, three epidermoid cysts, one simple cyst and a 18 years old boy with gynecomastia, two cases with intramammary lymph nodes, two myofibroblastomas, one leiomyoma of the nipple with breast tissue infiltration and six malignant breast tumors.

We present the most relevant cases of these mammographic studies of male breast pathology with special mention on gynecomastia and those cases hardly reported on the literature such as myofibroblastoma and leiomyoma of the nipple with breast tissue infiltration.

\section{SE}

\section{Gastrointestinal and abdominal radiology (04)}

\section{SPACE 1413}

Contribution of US and CT to the diagnosis of splenic abscesses in typhoid fever (report of 8 cases)

R. Allal (Presenter), B. Kastler, M. Benkalfat, O. Bouali, M. Y. Jeung, J. L. Dietemann; Strasbourg

Purpose: Salmonella typhi splenic abscesses (SA) are classically a rather rare complication of typhoid fever (TF). The aim of this study was to stress the role of US and CT to the diagnosis and follow up for these patients.

Methods: $\mathbf{4 0 0}$ patients with proven biological diagnosis of TF were systematically examinated by abdominal US. 20 patients with either persistent or recurrent symptoms (despite adequate treatment) or presenting acute lower thoracic pain, were performed again by US and CT with contrast media.

Results: In 8 patients, both imaging modalities revealed anomalies compatible with splenic abscesses; none of these patients had classical predisposing factors such as sickle cell disease. 7 patients were treated by splenectomy. In one case of small splenic abscess, repetitive US controls allowed follow up of total regression of abscess under medical treatment only.

Conclusions: SA are not so rare as previously described. Non invasive imaging modalities like US combined with CT allow the exact diagnosis and improve the prognosis by preventing a possible fatal issue like splenic rupture and peritonitis.

\section{SPACE 1414}

Radiographic manifestations of Crohn's disease

J. Andreu (Presenter), L. Casas, E. Grivé, S. Coll, S. Pedraza, J. A. Jimenez; Barcelona

The purpose of this exhibit is to present the typical and unusual radiological features in Crohn's disease and its complications.

Radiological examinations in 102 cases of Crohn's disease are presented. Atypical forms of radiological presentations with their pathological correlations are described.

The value of barium examinations and $\mathrm{CT}$ in the diagnosis of complications are discussed. CT adds valuable information in certain instances but is not necessary in every case.

\section{SPACE 1415}

Role of CT in the evaluation of gastric disease

J. Andreu (Presenter), J. A. Jimenez, S. Pedraza, A. Alvarez Castells, M. C. Sanchez, J. Alvarez; Barcelona

CT permits a correct visualization of the gastric wall and extramucosal extension of gastric lesions. The purpose of this exhibit is to evaluate the usefulness of CT in gastric diseases and to present the different CT features and their correlations with gastric lesions.

We reviewed the radiological findings in 52 cases of gastric process. Congenital, neoplastic and inflammatory etiologies are included.

Barium studies, endoscopy, surgical and pathological findings are correlated with CT findings.

Gastric wall thickening and mass effect are the most common findings. CT features in several cases can suggest the diagnosis and could be the only radiological sign of disease. 


\section{SPACE 1416}

Hyperdynamic portal hypertension - diagnosis and treatment M. Andrzejewska (Presenter), M. Januszewicz, M. Elwertowski, St. Goryń, O. Rowiński, B. Pruszyński, B. Michałowicz; Warszawa

Authors present $S$ case reports illustrating the diagnostic and therapeutic problems in management of portal hypertension caused by abnormal arterio-venous anastomoses.

In all cases US with Doppler studies and CT with angio-dynamic procedure were performed as a first, non-invasive method.

These studies showed the placement, width and patency of vessels, direction and level of blood-flow.

Angiographies, performed in all cases as the most specific method, showed the details of anatomy of anastomoses (fistulas). These enabled us to introduce the best way of treatment - embolization or surgical procedure.

\section{SPACE 1417}

Non-invasive diagnostic techniques in the evaluation of porto-systemic shunts

M. Andrzejewska (Presenter), M. Elwertowski, R. Pacho, B.Pruszyński, K. Zieniewicz; Warszawa

The studied material included 51 patients in whom for recurrent bleeding from esophageal varices 54 different porto-systemic shunts were performed (portocaval shunt in 31 cases, splenorenal shunt modo Linton in 6 cases, spleno-renal shunt modo Warren in 6 cases, mesenterica-caval modo Drapanas - 4 cases, other types -3 cases). The anatomical condition of each shunt, direction of blood flow and collaterals were diagnosed by US with Doppler studies and CT with dynamic-angio procedure.

Both methods allowed a simple and safe visualisation of porto-systemic anastomoses. CT is more sensitive and specific in diagnosis, especially of port-caval shunts, and can be used in early postoperative period. US is the useful method for long-term monitoring of the shunt condition.

\section{SPACE 1418}

Comparative study of dynamic CT and ultrasonic pulsed Doppler flowmeter on estimation of portal blood flow

Sachio Awai (Presenter), Shin Kimoto, Kötaro Yasui, Setsuko Istihara, Sumiyo Kuroki, Kaori Kobayashi, Yoshio Hiraki; Okayama

Purpose: To evaluate the portal blood flow, we performed liver dynamic CT and used a pulsed Doppler flowmeter in normal volunteers and examined the results of both methods comparatively.

Methods and Materials: In the dynamic CT Toshiba CT 900S was used. We administrated non-ionic hypotonic contrast medium by bolus injection in normal volunteers ( 16 males, 14 females) to a slice that allows a simultaneous grasp of the abdominal aorta and main portal vein for dynamic CT. We constructed time density curves of the abdominal aorta and main portal vein on the basis of picturers obtained, named the ratio of area of each steep rise portal blood flow coefficient. On the other hand, an ultrasonic pulsed Doppler flowmeter (Toshiba SSA 270A) was used for the same subjects, and the mean blood flow velocity ( $\mathrm{F}$ mean) and the mean blood flow volume ( $\mathrm{V}$ mean) of the main portal vein were determined.

Results: The portal blood flow coefficient was $0.46 \pm 0.18, F$ mean $0.21 \pm 0.06 \mathrm{M} / \mathrm{sec}, \mathrm{V}$ mean $1.01 \pm 0.45 \mathrm{~L} / \mathrm{min}$. A significant correlation was observed between the portal blood flow coefficient and values obtained with the ultrasonic pulsed Doppler flowmeter.

\section{SPACE 1419}

X-Ray check up after total gastrectomy (Roux-Y-Esophagojejunostomy)

M. Baldt (Presenter), Ch. Armbruster, W. Anzböck, H. Mosser, W. Hruby, K. Stellamor; Vienna

a) From Jan 1986 to Dec 1989110 patients underwent Roux-Y-Esophagojejunostomy after total gastrectomy. 32 patients are presented in a radiologic study regarding morphology and functional testing.

b) Long term follow up studies are performed by using double contrast method with Barium ( $350 \mathrm{ml}$ Prontobario HD, $6 \mathrm{~g}$ Duplotrast $\mathrm{Z}), 3$ to 36 months post operation.

c) We found 2 local recidives of tumor ( 1 only by endoscopy). 2 tight proximal anastomoses, caused by metastases. All proximal and distal anastomoses are well viewed. The average passage time from proximal anastomosis to the ileocoecum is 20 to 30 minutes. $(n=15)$.

11 patients showed an increased time: 2 with diarrhoea. 6 patients showed a decreased time; 1 with obstipation. Jejunoesophageal reflux was seen in 26 patients $(81 \%)$. Only 7 needed therapy for esophagitis. The feeding loop was viewed in 19 cases.

d) Barium double contrast examination seems to be useful for showing the feeding jejunal loop (56\%) in case endoscopy fails. The high number of jejunoesophageal reflux and variable passage times do not correspond with clinical complaint.

\section{SPACE 1420}

MRI of gastrointestinal tract: proposal of cost free, non invasive contrast medium

L. Balzarini (Presenter), S. Aime, L. Barbero, E. Ceglia, M. Fasano, R. Petrillo, J. Reyner, J.D. Tesoro Tess, R. Musumeci; Milano

Methods: After the occasional observation in a nourished baby of good contrast delineation of the upper GI tract, we tried a series of different commercial fresh and lyophilized milks both in experimental and in clinical tests. The laboratory evaluations were made with a $.5 \mathrm{~T}$ while imaging was performed with an $1.5 \mathrm{~T}$ magnet. After the selection of the most effective lyophilized product, prepared in conformity with commercial intructions, we examined a group of patients with gastric and rectal cancer.

Results: The most intense positive contrast effect, due to shortening of the T1 relaxation time, was obtained with Nidina 1 (Nestle'). This attitude is related to the concentration of the paramagnetic ions $(\mathrm{Mn}-\mathrm{Fe})$ binded in the macromolecules of milk, such as casein. The best imaging sequence was a spin-echo Tl.w. After ingestion of milk a good delineation of the stomach with sufficient distribution in duodenum and in the very proximal bowel was achieved as well as a good depiction by enema of rectum and recto-sigmoid junction.

Conclusions: The practical value of this paramagnetic complex depends on its non invasivity, economicity and acceptability thus we suggest the use of the lyophilized milk "Nidina 1 " in patients with gastric and rectum-sigmoid cancer in order to increase the diagnostic efficacy of MR.

\section{SPACE 1421}

US serial evaluation of volumes of hepatic metastases: growth rates of untreated nodules

Alberto Banderali (Presenter), Lorenzo Egildo Derchi, Pierluigi Brovero, Patrizia Votano; Genova

The quantitative study of neoplastic growth patterns is the ground for a correct clinical approach to patients with malignant tumour.

We followed-up the growth of 23 hepatic metastases (HM) in 16 patients measuring the three orthogonal diameters of any lesion by means of US, calculating their volumes and plotting the results in semilogarithmic scale. Doubling times (DT) were also calculated, as an index of growth speed.

We obtained values of DT scattered from 13 to 770 days.

In the fast growth group (DT less than 50 days) we found all the HM from pancreas and stomach and about $50 \%$ of $\mathrm{HM}$ from sigmoid colon and rectum. In the intermediate growth group (DT more than 50 and less than 100 days) we found the remaining $50 \%$ of $\mathrm{HM}$ from sigmoid colon and rectum and $67 \%$ of HM from breast. In the slow growth group (DT more than 100 days) we found the remaining $33 \%$ of $\mathrm{HM}$ from breast and $\mathrm{HM}$ from small intestine carcinoid, Vater's ampulloma and parotid gland cylindroma.

Considering the obtained results, we stress the importance of a correct volumetric study during US follow-up of patients with untreated HM.

\section{SPACE 1422}

$U$ S in the volumetric evaluation of hepatic metastases' response to chemotherapy

Alberto Banderali (Presenter), Lorenzo Egildo Derchi, Patrizia Votano, Pierluigi Brovero; Genova

The accurate evaluation of the response of hepatic metastases (HM) to chemotherapy is still an unattained goal.

We followed-up $60 \mathrm{HM}$ in 31 patients during chemotherapy, measuring the three diameters and calculating their volumes. The period of follow-up was longer than 1 year in $52 \%$ of patients and the number of observations was more than 3 in $58 \%$ of $\mathrm{HM}$. The curves of growth were completed with data concerning chemotherapeutic courses. $90 \%$ of $\mathrm{HM}$ derived from a gastroenteric primary tumour, the remaining $10 \%$ from breast tumour.

In the initial period of chemotherapy we observed shrinkage in $55 \%$ of $\mathrm{HM}$, slower growth in $40 \%$ and unchanged growth rate in $5 \%$. In the late period of chemotherapy we observed reduced or absent response in $64 \%$ of the HM and a better response in $36 \%$. At the end of the therapeutic course the prevailing feature was faster growth or new growth in $75 \%$ of $\mathrm{HM}$; finally, in the period after the sixth month from the end of the chemotherapeutic course the prevailing feature was faster growth rate $(58 \%)$. We stress the importance of a correct volumetric approach to HM during chemotherapy as a precious tool for evaluating its effects. 


\section{SPACE 1423}

Initial diagnosis and follow-up of metastatic endocrine Gl-tumors R. Benning (Presenter), J. Froelich, Ch. Neuhaus, K. J. Klose, R. Arnold; Marburg

Eighty patients with endocrine GI-tumors have been followed up with abdominal CT, chest X-ray and sonography in three month intervals. Most frequent primary tumor sites were ileum (27 pts.) and pancreas (32). Rare localisations were stomach (3), duodenom (2), jejunum (3), colon (2), rectum (1) and retroperitoneum (1). Imaging failed diagnosis of primary tumor site in 19 cases. In 10 of these cases surgery or autopsy was identifying the primary tumor.

Liver metastasy was most common (74). 17 patients revealed extraabdominal metastases (skeletal 8 , ovarian 3 , CNS 3 , breast 1 , subcutaneous 2 ).

Compared to the well-described Krukenberg's tumor there are only few reports about CNS-involvement in literature so far.

Generally chest $x$-ray, sonography and computed tomography are sufficient for diagnosis and follow up of endocrine Gl-tumors. Additional procedures should be performed only in certain cases. Based on our experience we are discussing the most effective procedure of initial diagnosis and follow up.

\section{SPACE 1424}

Cholecystolithiasis in women in Czechoslovakla - ultrasonographic study from Slovakla - town Trenčín

J. Bielik (Presenter); Trenčin

In the years 1986-1989 a collective of 2102 girls and women at the age of 15-80 years was examined ultrasonographically. Cholecystolithiasis occurred in $17,21 \%$, with a cholecystectomy group equal to $23,12 \%$. Cholecystolithiasis was present in $8,19 \%$ as asymptomatic, in $17,67 \%$ as atypical and in $75,0 \%$ as typical form. In mothers cholecystolithiasis was documented in $15,52 \%$ and in fathers in $3,17 \%$. Cholecystolithiasis occurred within fat women in $28,99 \%$, while within normal ones - $11,31 \%$; childless women $-6,7 \%$, one birth $21,81 \%$, two and more births $-23,33 \%$; association with diabetes mellitus was in $36,91 \%$, without diabetes $-15,85 \%$; using hormonal contraception $-14,49 \%$, without using hormonal contraception $-17,31 \%$; with smoking $-11,5 \%$ and with not smoking $-17,96 \%$; with drinking coffee $-15,24 \%$, with not drinking coffee - 18,24\%: with intellectual work (over 19 years) $-18,7 \%$ and with manual work $-25,6 \%$

We stated these risk factors: 1. mothers genetic influence, 2. age, 3. obesity (mainly in the early and middle age), 4 . gravidity - mainly in the early age, markedly numerous and 5 . diabetes.

Neither hormonal contraception nor smoking were proven to be risk factors. Coffeine is the possible protective factor of the beginning of cholecystolithiasis (by the literature it is the first occurrence).

\section{SPACE 1425}

Imaging findings in peliosis hepatitis, a review of four patients D. Bodnar (Presenter), L. Henry, G. Genin, P.-J. Valette; Lyon

Goal: 4 cases of peliosis hepatitis have been studied by different imaging modalities including CT, DSA, and MRI in order to define some potential specific findings allowing a precise diagnosis. The pathological appearance of this rare entity is characterized by the presence of multiple permanent bloodfilled cysts of variable size.

Methods: After US detection, CT with contrast agent injection, DSA and MRI were used in all patients and the data correlated with the pathological findings obtained by aspiration cytology $(n=2)$, percutaneous biopsy $(n=1)$, and surgical biopsy $(n=1)$.

Results: US demonstrated nodular lesions, which were confirmed by CT as hyperdense lesions after contrast media bolus injection, without any finding in favor of malignancy or benignity. The MRI appearance evoked potentially malignant lesions based on the heterogeneous signal intensity without major increase on T2-weighted images. On the other hand, DSA appeared as the more adapted imaging modality to approach the right diagnosis, demonstrating an early opacification lasting at the venous phase.

From these four patients, DSA could be suggested as the more specific imaging modality to orient the diagnosis and the pathological evaluation.

\section{SPACE 1426}

Treatment of small hepatocellular carcinomas by US-guided percutaneous ethanol injection (PEI), results in 15 patients

Maurizio Borghetti (Presenter), Giampaolo Benelli, Roberto Bonardi; Crema

Purpose: This report shows the results obtained by US-guided PEI in 15 patients affected by small $\mathrm{HCC}($ diam $3 \mathrm{~cm})$.

Methods: PEI consisted in 2 weekly sessions ( $\max 11$ for each nodule). The dye (2-4 cc per session) was injected by means of a US-guided fine spinal needle. In addition to nodule US changes, the criteria confirming the success of this treatment were:

a) absence of malignant cells at the final FNAB; b) lack of enhancement during the TC arterial phase and formation of a peripheric hyperdense ring. US, TC and the AFP levels were used for follow-up.

Results: All patients are still alive and in only one case, recurrencies of the neoplasia were reported. The follow-up varies from 1 to 24 months. Nodules have progressively reduced and in some cases they have disappeared. US shows a nearly constant posterior acustic shadow revealing the postnecrotic fibrosis. Treatments have been usually well tolerated by all patients.

Conclusions: Since surgery is influenced by the conditions of cirrhotic patients, PEI, being easily available, can be a successful alternative for small HCC. The main disadvantages of PEI are due to the site of the tumor and the hardness of the cirrhotic liver which might cause deviations of the fine needle.

\section{SPACE 1427}

Diagnostics and therapy of malignant peritoneal mesothelioma (MPM) E. Brenčič (Presenter), A. Višnar-Perović, M. Stanovnik; Ljubljana

Five patients with malignant peritoneal mesothelioma were treated; four of them for a period of two years.

The fifth patient who was treated for eight years underwent surgery several times. Four patients had insignificant clinical pictures. Computed tomography (CT) examination revealed typical irregular thickening of peritoneum and tumor of omentum. The diagnosis was later confirmed by the cytologic evaluation. In one case the MPM diagnosis was confirmed by histologic evaluation after surgery. All patients were treated with systematic cytostatic therapy combined with radiotherapy. The success of the therapy was monitored by ultrasound (US) and CT. The response to the treatment was satisfactory. The remissions were of different duration. Four of five patients died. The results of diagnosis of MPM with US and CT and the success of therapy for five patients are described.

\section{SPACE 1428}

Primary adenocarcinoma of the jejunum and lleum, radiologic Imaging of 10 cases

M. R. Calero (Presenter), C. Lopez, E. Añorbe, M.S. Gallego, P. Manjon, L. Abad; Madrid

Primary adenocarcinoma of the small bowel is an uncommon tumor, accounting for less than $1 \%$ of all gastrointestinal neoplasms.

We reviewed 10 cases of jejunum and ileum malignancies diagnosed from 1977 to 1989 in our institution, with histopathologic correlation. They were 4 men and 6 women. Ranging age from 22 to 82 years (mean 59 years). More usual clinical symptoms were abdominal pain and weight loss. Topographic localization was jejunum in 6 cases and ileum in 4 cases.

Radiologic imaging was strongly suggestive of malignancy in 7 cases: 6 showed a typical annular "napkin-ring" configuration, one of them simultaneously presenting 2 lesions in jejunum; and the other displayed destruction of the mucosa with a large ulcerating lesion. In 3 cases radiological findings were atypical: mucosal diffuse involvement with gross folds of whole small bowel; lesion of terminal ileum suggestive of Crohn's disease, and one patient had no radiologically detectable abnormalities.

\section{SPACE 1429}

Differential diagnosis of the gastrointestinal ultrasonographic "target" sign

T. Cammarota (Presenter), G. Rossi, G. Cavalot, M. Sandrone, C. Reviglio: Torino

Aim of our study is to define the different semiological aspects of the gastrointestinal ultrasonographic "target" sign.

The purpose of this study is therefore to search for the various features of the "target" that can help in the differential diagnosis of disease that cause bowel thickening.

The ultrasound examination was performed with $7 \mathrm{MHz}$ linear probe, together with a plain XR of the abdomen in a large number of patients (over 100) with acute abdomen, intestinal occlusion or subocclusion.

Ultrasonographic findings were afterwards compared to the radiological, surgical or artificial diagnosis.

The "target" sign found in Crohn's disease, in lymphoma, in peritoneal carcinomatosis, in intestinal infarction, in volvulus, in intussusception, in acute appendicitis have different morphological features (radial target in lymphoma, irregular and thick target in carcinoma, etc.) that, together with clinical data, have shown a high diagnostic specificity. 


\section{SPACE 1430}

CT and US signs of midgut malrotation and volvulus

J.D. Casas (Presenter), R. J. Perea, J. Bechini, A. Rivas, L. Inaraja, J.Piqueras; Barcelona

Purpose: The aim of this study was to present the CT and US findings of midgut malrotation, with and without volvulus, and to show by US a well known $\mathrm{CT}$ sign of volvulus in intestinal malrotation (whirl-like encircling loops of bowel around the mesenteric vessels).

Methods and Materials: Five patients (age range: $|6-3|$ years) with midgut malrotation (one of them with volvulus) were studied with CT and US (3.5- $\mathrm{MHz}$ transducer). Diagnoses were confirmed surgically in volvulus, and with barium studies in all patients.

Results:

Abnormal position of the jejunum:

Abnormal position of the cecum:

Abnormal disposition of the mesenteric vessels*:

Dilatation of proximal midgut:

Whirl-like encircling loops of bowel around the

mesenteric vessels:

Numerous vessels in the mesentery:

(*: the superior mesenteric vein was seen to lie:

- to the left of the superior mesenteric artery

- on the left ventral aspect of the SMA

- immediatly anterior and slightly to the right of the

SMA

$\begin{array}{ll}\text { CT } & \text { US } \\ 5 & 0 \\ 4 & 0 \\ 5 & 5 \\ 1 * * & 1^{* *} \\ 1^{* *} & 1^{* *} \\ 1^{* *} & 0 \\ & 1^{* *} \\ & 2 \\ & 2)\end{array}$

\section{SPACE 1431}

Radiologic evaluation of the vertical banded gastroplasty for morbid obesity

J. D. Casas (Presenter), R. Perez-Andres, M. Tenesa, A. Alastrue, M. Rull, X. Formiguera; Barcelona

Purpose: The aim of this study was to present the routine used in our series of morbidly obese patients treated with vertical banded gastroplasty (VBG), and to show the results with the most representative images.

Methods and Materials: 70 patients (age average: 36.4 years) with morbid obesity (weight average: $126 \mathrm{~kg}$ ) were treated with VBG. All patients were routinely studied preoperatively and postsurgically $(6,12,18,24$ months after VBG) with upper GI series and US. CT were only used when a leak was suspected.

Results:

Fatty liver:

47

Gallstones:

26

Cholecystectomy (before or in VBG):

Developed after VBG:

Extragastric leak:

Staple-line disruption:

Restapling because regained weight:

Outled obstruction:

Food/Bezoar formation:

Mucosal intussuspection:

Erosion of the band:

$\begin{array}{rr}18 & 26 \\ 8 & \\ & 2 \\ 5 & 16 \\ 2 & 5 \\ 1 & \\ 2 & \end{array}$

\section{SPACE 1432}

Intraoperative detection of satellite nodules in encapsulated HCC L. Casas (Presenter), S. Coll, R. Boyé, H. Allende, J. Balcells, D. Sureda; Barcelona

Purpose: To evaluate the correlation between the histopathologic study of resected hepatocellular carcinoma (HCC) and findings from preoperative and intraoperative ultrasonography (US), with special emphasis on the detection of satellite nodules.

Methods and Materials: Preoperative US was performed on 112 patients affected of HCC. In 12 of the 21 patients subjected to tumor resection the following pre and intraoperative US parameters: size, echostructure, capsule and presence of satellite nodules were compared with the histopathologic specimen.

Results: The correlation between US studies and specimens concerning tumor size (mean $4 \mathrm{~cm}$ ) was $100 \%$. Hypoechogenicity correlated with intratumoral necrosis in $83 \%$ of preoperative and $100 \%$ of intraoperative US. Peritumoral halo was detected in $100 \%$ and $67 \%$ of cases by pre and intraoperative US, respectively, and correlated with the capsule in the tumoral specimen. In 6 complete encapsulated HCC, histological study demonstrated satellite nodules in 4. In preoperative US no cases showed nodules and in intraoperative US nodules were detected only in one. Our results indicate that US demonstrates great effectiveness in determining the size and echographic patterns of the tumor. However, low sensitivity was observed in the detection of satellite nodules. We conclude that the surgical resection should include a safe peritumoral margin even though the tumor is well-defined echographically.

\section{SPACE 1433}

Radiologic approach to polypoid esophageal tumor

L. Casas (Presenter), J. Andreu, P. Vicente de Vera, E. Castellà, M. C. Sanchez, E. Grivé: Barcelona

Polypoid lesions are rare presentation forms of esophageal tumor. Radiological findings with pathological and surgical correlations in polypoid tumors of the esophagus are presented.

We reviewed 92 cases of esophageal neoplasm and found 15 polypoid tumors. These included carcinosarcoma ( 6 cases), squamous cell carcinoma ( 2 cases), adenocarcinomas ( 2 cases), Leiomyosarcoma (1 case), oat-cell carcinoma (1 case) and metastasis ( 3 cases).

In barium examinations, bulky intraluminal masses are found in all cases. CT may show different densities in the tumor that can help in the differential diagnosis.

\section{SPACE 143}

Transcatheter arterial chemoembolization (TAE) in operable hepatocellular carcinoma (HCC); post-surgical, histopathological evaluation and 2 years follow-up in 5 patients

M. Castrucci (Presenter), A. Vanzulli, R. Mellone, S. Sironi, G. Ferrari, C. Marenghi, A. DelMaschio; Milan

Purpose: TAE is generally considered to be an effective palliative treatment in inoperable HCC. TAE has also been performed in operable cases, in order to reduce the chances of recurrence. The aim of the present study was to evaluate the histopathological changes and midterm clinical results in resected HCCs after TAE.

Methods: We performed TAE, by intra-arterial injection of Lipiodol and chemotherapeutic agent (Adriamycin), followed by embolization with Spongostan, in 5 patients with operable HCC. All patients underwent subsequently partial hepatectomy. Follow-up was performed by monitoring AFP monthly and by US.

Results: Histopathological analysis of the resected HCCs showed in all HCCs a thick fibrous capsule, complete necrosis of the main tumor in $4 / 5$ cases; 1 HCC remained viable and tumor cells were found in few daughter nodules surrounding the tumor. In one case there were viable tumor emboli in the portal vessels around the tumor. From the clinical point of view, only 1/5 patients died after 13 months because of progression of cirrhotic disease.

Conclusions: In resectable HCC, TAE could be useful in preventing an increase of the tumor, producing capsule thickening and reducing the chances of local recurrence, thereby making surgery safer, avoiding a spreading of portal neoplastic emboli.

\section{SPACE 1435}

Hepatocellular carcinoma: comparative study between US, CT and MR J. A. Clavero (Presenter), M. C. Ayuso, C. Bru, J. Bruix, R. Vilana, X. Tomás; Barcelona

1. We have made a double-blind prospective study of the US, CT and MR findings observed in eighteen consecutive patients who suffered from hepatocellular carcinoma (HCC).

2. Eighteen patients diagnosed of HCC were studied during four months. Seven out of them were uninodular and the rest of them multinodular. They were evaluated by US, unenhanced and enhanced CT scans and MRI using TI and $\mathrm{T} 2$ weighted spin-echo secuences. These examinations were performed to assess tumoral characteristics (size, fibrous capsule and internal architecture), intra and extrahepatic extension and to depict the relationship of hepatic vascularisation to the malignancy.

3. Overall accuracy of US, CT and MR was $\mathbf{9 4 . 4 \%}$. False negatives were seen in small size tumours. In our study, MR has proved to be superior to CT and US providing tumoral tissue characterization, but it could not be proved significantly better than those strategies together in assessing tumoral extension.

\section{SPACE $\underline{1436}$}

Esophagus in A.I.D.S. patients

G.P. Cornalba (Presenter), N. Franceschelli, D. Segre, G. Matacena, R. Bianco, G. Damiani, A. Malerba; Milano

Purpose: The authors study the usefulness of the radiographic procedures for the identification of the esophageal lesions in A.I.D.S. patients with dysphagia. In a significant percentage of cases, radiographic patterns are helpful to make a differential diagnosis between infections and neoplasms. 
Methods and Materials: 250 patients underwent double-contrast esophagography.

Results: Candida infections are the most frequent cause of esophagitis in patients with A.I.D.S.; the radiographic appearance is characteristic and it includes diffuse ulcerations, cobblestoning, plaques and thickened folds.

Radiographic patterns of C.M.V. esophagitis are also characteristic with focal ulcers and well defined areas of edema. Giant ulcers may often be seen at the gastroesophageal junction.

In Herpes simplex esophagitis, discrete ulcerations with no specific character may be seen.

Neoplasm can be radiographically separated from infections.

Conclusions: The radiographic appearance of esophageal lesions is important and is useful for a differential diagnosis in a significant percentage of cases.

\section{SPACE 1437}

\section{Ultrasound in acute appendicitis}

B. Cortina (Presenter), N. Alegre; Madrid

Purpose: We make a prospective study on the usefulness of the U.S. in acute appendicitis.

Methods and Materials: We included eighty-two patients attended in the emergency ward with a diagnosis of acute appendicitis made just on clinical basis. We used a $7.5 \mathrm{MHz}$. transducer applied on the right iliac fossa and a $3.5 \mathrm{MHz}$. to explore the rest of the abdomen.

Results: By US the appendix was identified in $63.3 \%$ of cases, an appendicolithiasis was seen in $3.33 \%$, a periappendicular abscess in $5 \%$ and free peritoneal fluid in $3 \%$.

The classic US pattern of acute appendicitis was found in $72.72 \%$ of cases, the hypoechoechoic form in $18.18 \%$ and periappendicitis in $9.09 \%$

All cases with an ultrasonographic diagnosis of appendicitis were surgically confirmed.

In our experience the US showed a diagnostic reliability of $64 \%$ and in $8 \%$ it led to a diagnosis of another pathology.

\section{SPACE 1438}

\section{Biliary obstructions: management with metallic mesh stents}

Turhan Cumhur (Presenter), Tulay Oicer, Serdar Karakose, Ahmet Mavis, Erkin Aribal, Bedrettin Selcuk, Ismet Tas, Cumhur Ceken; Ankara

Purpose: Transhepatic biliary endoprosthesis placement is well established procedure for the relief of obstructive jaundice. Recently stainless steel biliary stents became a new alternative to the conventional endoprostheses because of the high frequency complication of the latter. We placed self-expandable metallic mesh stents for the treatment obstructions.

Methods and Materials: Self-expandable mesh stents have been placed in 13 patients. Ten patients had malignant obstruction and 3 patients had benign strictures as a result of several surgical interventions to the biliary system. We placed Wall stents (Medinvent, Switzerland) with diameter of $30 \mathrm{~F}$ when fully open.

Results: No major complications observed. The mean follow-up period was 6 months and during the follow-up all the stents were patent. One patient died of his disease on the 39 th day after the procedure.

Conclusions: The initial result suggests that metallic stents can be used successfully instead of conventional endoprostheses which have relatively small diameter and high complication rate. Also the ability of the placement of Wallstents through a $7 \mathrm{~F}$ catheter reduces the risks of the procedure. Our results are encouraging. We believe that further studies will clarify the long-term success.

\section{SPACE 1439}

\section{Gastric metastasis of breast cancer: radiologic features}

T. H. Dao (Presenter), T. Nguyen-Tan, A. Scherrer, R. J. Salmon, J. Girodet; Paris

Purpose: To describe the various radiologic features of gastric metastases of breast cancer which are rare but not exceptional because of the high incidence of breast cancer.

Methods and Materials: X-ray, sonographic and computed tomography features of 12 cases of biopsy-proven gastric metastases of breast cancer are reported.

Results: 2 radiologic patterns can be described:

1. diffuse gastric metastases are more common, with diffuse thickness of the gastric wall, related with polyvisceral and peritoneal involvement.

2. apparently isolated nodular gastric metastases, mimicking primary gastric tumor, possibly related with ductal carcinoma and hematogeneous spread to the stomach.

Conclusions: Knowledge of radiologic features of gastric metastasis contributes for early diagnosis and may improve survival by appropriate treatment.

\section{SPACE 1440}

Ultrasonography in the differential diagnosis of ovarian tumors F. del Castillo González (Presenter), J. M. Fernandez Gallardo, N. Marcos del Rio; Madrid

182 ovarian tumors are studied for establishing the echographic characteristics and suggesting a specific diagnostic.

Women are studied with a 3.5 and $5 \mathrm{MHz}$. transducer. Echographic findings are compared with surgical pieces. Tumor echogenicity (anechoic, hypoechoic or hyperechoic) and structure (homogeneous, inhomogeneous) are analyzed. Septal appearance (thin $<3 \mathrm{~mm}$ and thick $>3 \mathrm{~mm}$ ), parietal nodules ( $(0\rangle$ than $2 \mathrm{~cm}$ ) and calcification frequency are also studied. We note other features like ascitis, bilaterality, good delimitations by capsule, etc.

We show echographic features in each tumoral group: epithelial tumors (C.Ad., C.adca.), stroma gonadal tumors, germinal cell tumors (teratoma, etc.) mesenchymal tumors (fibromas, thecomas, etc.), metastasis, etc.

As all of you know, echography is a high fyability method for detecting masses, but not so much for establishing a diagnosis. We propose you some echographic features that you can use in differential diagnosis of ovarian tumors.

\section{SPACE 1441}

\section{Ultrasound in gastrointestinal tract diseases}

J.L. del Cura (Presenter), A. Martinez, J.C. Ruiz, A. Aguinaga, E. Astigarraga, L. F. Vidales, S. Garcia; Bilbao

1. In order to describe the differential ultrasound (US) signs in digestive tract diseases, findings in 509 patients are presented.

2. The cases include 160 neoplasms, benign and malignant, 10 diverticulitis, 11 Crohn's diseases, 8 unspecific ileitis, 4 ulcerative colitis, 1 tuberculous enteritis, 3 ischemic colitis, 1 mesenteric vein thrombosis, 211 appendicitis, 5 mucoceles, 3 appendiceal intussusceptions, 47 intestinal intussusceptions, 11 gastroduodenal ulcers, 2 afferent loop syndromes, 1 afferent loop intussusception, 3 intestinal hematomas, 15 hypertrophic pyloric stenosis, 2 postpancreatitic duodenitis, 2 hernias, 2 duplication cysts and 1 duodenal diverticulum.

$3.5,5$ and $7.5 \mathrm{MHz}$ probes were used. Only in 53 tumoral patients the lesion was known before the US examination.

3. US allowed us to localize the lesion and guide the diagnosis in all the cases. It can be an useful tool for patients with abdominal pain. Recognition of gastrointestinal pathology by US may save time and money in the workup of such patients.

\section{SPACE 1442}

Intrahepatic arteriovenous shunts: duplex sonography and color flow imaging

L. Donoso (Presenter), A. Martínez-Noguera, E.Sanchis, Y.Pallardó, C. Traid; Barcelona

Purpose: To demonstrate the usefulness of doppler sonography and color flow imaging in the diagnosis of intrahepatic arteriovenous shunts.

Methods and materials: We have used duplex sonography and color flow imaging to diagnose eight patients with intrahepatic arteriovenous shunts (two cases of Osler's disease, three post-biopsy hepatic arterio-portal fistulae and patent spontaneous arterio-portal shunts in three cirrhotic patients).

Results: Diagnostic findings include: morphologic changes (intrahepatic solitary cystic lesions, segmental dilatation of portal vein, dilated and tortuous hepatic arteries and dilated hepatic veins) and alterations of flow dynamics (Hi-velocity bidirectional systolo-diastolic flow, turbulent flow and increased reversed flow in the involved portal vein). Demonstration of these findings by color and duplex sonography is easy and can obviate further investigations.

\section{SPACE 1443}

Arteriography and portography CT of hepatic neoplasms: our experience

Roberto Dore (Presenter), Luisella Rota, Giuseppe di Giulio, G. Carlo Barazzoni, Enrico di Maggio, Elisabetta Colombo, Rizzardo Anguissola; Pavia

Purpose: To improve radiologic presurgical assessment of hepatic neoplasms we have evaluated technique, findings, accuracy and limits of CT angiography and portography.

Methods: From January 1989 to December 1990 we have performed intraarterial CT in 33 patients affected by primitive or secundary hepatic neoplasms. 10 cases were porto-CT, 23 were arterio-CT. All patients previously underwent $U S$ and angiography, 23 patients i.v. angio-CT, 12 RM, 26 percutaneous fine needle biopsy. These methods did not exclude surgical treatment. Subsequently 15 patients underwent surgery or laparotomy; the remainders were periodically observed.

Results: Interpretation of arterio-CT findings was difficult in $50 \%$ of cases: 
transient vascular contrast enhancement caused images not always demonstrated for localizing tumor set. The accuracy of portal CT has been superior to all diagnostic methods in regard to number, seat and size of lesions. The results of both methods decreased in patients with arterial anomalies. Intra-arterial CT has been useful to exclude surgery in $30 \%$ of patients not previously excluded by US or conventional CT.

Conclusions: Intra-arterial-CT is useful in presurgical evaluation of hepatic tumor. Hepatic arterio-CT is better than porto-CT to recognize neoplastic nature of lesions; we prefer porto-CT in evaluation of size, number and seat of lesions. When angiography demonstrates vascular anomalies results of intraarterial-CT decrease.

\section{SPACE 1444}

\section{CT of less severe forms of colonic diverticulitis}

E. Doringer (Presenter), P. Sattlegger, R. Forstner, H. Schmoller; Salzburg

Purpose: The value of CT in less severe forms of colonic diverticulitis is determined in a prospective study.

Methods: 33 patients ( 20 female, 13 male, mean 71 years) with clinically suspected diverticulitis (left lower quadrant pain, leukocytosis, elevated erythrocyte sedimentation rate) were studied prospectively by CT. CT examinations were compared with contrast enema in 24 patients and with endoscopy in 6 patients. The results are proven by histologic specimen in 9 cases, the rest by the clinical course.

Results: The predictive value of symptoms, such as thickening of the colonic wall $(86,6 \%)$, inflammatory changes of the pericolic fatty tissue $(87,5 \%)$, the presence of diverticula $(73,3 \%)$ and abscess formation $(100 \%)$ were examined separately and their significance was evaluated. True positive results by CT were reached in $20 / 21$ cases (sensitivity $=95,2 \%$ ), true negative findings in $9 / 12$ (specificity $=75,0 \%$ ). The results of CT examinations were compared with those of contrast enemas and/or endoscopy. The number of cases was too low to achieve statistic significance but the relatively high percentage of questionable results $(7 / 24=29 \%)$ shows the difficulties inherent in these methods.

Conclusions: Our study shows that CT is a good means to demonstrate even less severe forms of colonic diverticulitis with sufficient reliability.

\section{SPACE 1445}

Diagnosis and differential echographic diagnosis of ascites

F. Draghi (Presenter), R. Mandrioli, A. Kluzer, F. Ferrozzi, G. Ferrozzi; Pontremoli

Ascites is the result of varius ethiopathogenetic causes, it becomes echographically evident only when it is more than $30,40 \mathrm{cc}$.; for lower values evidence is obtained only with paracentesis. We have reviewed retrospectively 150 echographic examinations of patients with ascites, in order to determine, through direct and indirect signs the cause of the disease. It was found that for 90 cases, out of those reviewed, the etiology was due to cirrhosis, of these $40 / 90$ presented evident echo-pathognomonic signs such as: portal hypertension, collateral vascular bed, megalosplenia; for 6 the cause was due to portal thrombosis of which 4 on neoblastic basis, in all of them the obliteration of vessel appeared evident; in $\mathbf{4}$ cases the cause was a phlogistic process and the ascites was corpuscolated; constrictive heart failure was noted in 3 cases and echography showed a clear dilation of the hepatic veins; a neoblastic pathology was found to be the etiology of 47 cases reviewed, of which 28/47 showed evident typical signs (such as ascites saccatus in the absence of any previous surgical operations, peritoneal vegetation, diffuse ascites except for the Douglas bay). The retrospective review of 150 echo-examinations performed in the half of 1990 shows indicant echo-signs in about $40 \%$ of the cases reviewed and the signs appear to be more frequent in the neoblastic, in the inflamed and cardiac ascites as well as in the portal venous thrombosis. The research of these signs, which are sometimes undervalued, enable us to reach, in a considerable number of cases, a diagnosis, thus avoiding further tests, which often result useless and expensive.

\section{SPACE 1446}

Allergic and anaphylactic reaction during a double contrast $X$-Ray examination of the gastroenteric tract

F. Draghi (Presenter), P. Simonelli, G. Ferrozzi; Pontremoli

The first symptoms of anaphylactic reactions, though seldom occurring during an X-ray examination, generally become manifest with an unusual developement of urticaria shortly after assumption of the contrast medium. The methylparaben, a preservative contained in the barium mixtures, is likely to be the responsible allergen. The male patient P.G., under our observation before being submitted to an X-ray study of stomach and duodenum presented, in his personal and family anamnesis no occurrence of allergic diseases. The examination was performed, as usual, in a double contrast without using the hypotonic agent. At about 20 minutes from the assumption of the barium mixture the patient developed urticaria all over his body but mostly on his face, trunk and arms. Emergency treatments were immediately applied. The patient soon recovered. In the following radiograms performed, a rapid transit of barium into the small bowel was noted; a likely consequence of histamine release. The case reported proves how idiosyncracy phenomena occurring during X-ray examinations of the digestive apparatus are caused by the sulphate of barium mixture and not by the hypotonic agent. Therefore we consider it advisable to maintain an antiallergic kit at hand in the diagnostic suite, for any emergency.

\section{SPACE 1447}

\section{Gallstone lleus: sonographic diagnosis}

E. Elizagaray (Presenter), F. Sáez, A. Martínez, E. Tejera, J.Calonge, L. Larrea, T. Grande; Bilbao

The classic gallstone ileus triad (pneumobilia, obstruction and ectopic gallstone) can be diagnosed by abdominal plain films and sonography. We compare the findings of these two techniques in 12 patients with gallstone ileus.

Results:

\begin{tabular}{llll}
\hline & Pneumobilia & Obstruction & Ectopic gallstone \\
Plain films & 8 & 7 & 5 \\
Sonography & 10 & 9 & 7 \\
\hline
\end{tabular}

No cases showed clear vesicular lumen on ultrasound: in 3 cases a doublearch-shadow image was identified and in 9 cases an echogenic line with acoustic shadowing was seen in the gallbladder bed. Four patients had a previous sonographic study which demonstrated a gallbladder of normal lumen, with gallstones. This appearance changed to nonvisualization of the gallbladder lumen.

Conclusions: Sonography is superior to abdominal plain films to evaluate the classic triad of gallstone ileus. Visualization of a normal vesicular lumen helps to rule out the diagnosis of gallstone ileus.

\section{SPACE 1448}

\section{Ultrasonography of intrahepatic arteriovenous fistulas}

M. Elwertowski (Presenter), M. Andrzejewska, P. Rajszys, M. Januszewicz, J. Pawlak, O. Rowinski, K. Zieniewicz, B. Michalowicz; Zalesie Gorne

Fistulas between hepatic artery and hepatic veins or portal vein branches are seldom encountered and difficult to diagnose diseases with potentially poor prognosis. Ultrasonic Doppler study with evaluation of hepatic vessels flow was performed in 6 patients with various connections of hepatic artery with venous and portal systems. The results were confirmed with DSA, CT, surgery ( 3 cases) and autopsy ( 1 case).

In 3 patients when colour Doppler was available diagnosis of 2 fistulas between hepatic artery and hepatic veins as well as one fistula with portal vein trunk was stated. In one patient plain Doppler examination was sufficient for making diagnosis of connection between splenic artery and splenic vein. In 2 patients Doppler study could not state the reason for huge portal vein collaterals, that showed to be related with arterioportal fistulas - one demonstrated in DSA, the other diagnosed only on autopsy.

The results show that only colour Doppler study allows definite ultrasonic diagnosis of hepatic vascular fistulas. They should be suspected in every case of abundant portal vein collaterals with no apparent reason (liver pathology, portal vein thrombosis), or hepatic veins malformations.

\section{SPACE 1449}

Tc-99m-DISIDA hepatobiliary imaging in patients with liver transplantation: correlation with liver biopsies

C.M. Engeler (Presenter), C. C. Kuni, R. Nakhleh, C. E. Engeler, R.P. duCret, R. J. Boudreau; Minneapolis, MN

Purpose: Comparison of Tc-99m-DISIDA hepatobiliary scintigraphy with biopsy results in patients after liver transplantation.

Methods and Materials: Seventy-six Tc-99m-DISIDA hepatobiliary studies with corresponding liver biopsies were reviewed. Radionuclide scintigrams were evaluated for uptake (normal $=$ minimal cardiac blood pool activity at the 10 minute image) and excretion (normal = prompt decrease of activity subsequent to the 15 minute image). Biopsies were graded on scales of a) 0 to 6 for hepatocyte damage $(0=$ normal $), b) 0$ to 12 for findings of cholestasis $(0=$ normal) and c) 0 to 9 for rejection $(0=$ normal).

Results: Sixty/seventy-five $(80 \%)$ showed normal Tc-99m-DISIDA uptake and $65 / 76(85 \%)$ showed delayed excretion. Forty-nine/seventy-six (64\%) demonstrated a combination of normal uptake and delayed excretion. At 3, 8, 14 and 31 days after transplantation $71 \% ; 79 \% ; 82 \%$ and $78 \%$ had normal uptake and $0 \% ; 4 \%, 3 \%$ and $6 \%$ had normal excretion. Comparison of biopsies with scintigrams showed a positive association of hepatocyte damage scores with uptake $(p<.001)$ and of cholestasis/rejection scores Tc-99m-DISIDA excretion $(\mathrm{p}=.01 / 0.05)$. 
Conclusions: Tc-99m-DISIDA scintigraphy of liver transplants usually demonstrates normal tracer uptake with delayed excretion. Comparison with biopsy results suggests that cholestasis/rejection can be differentiated from pure hepatocyte dysfunction.

These findings will be illustrated with examples from liver scintigrams and biopsies.

\section{SPACE 1450}

\section{CT patterns of peritoneal carcinomatosis as predicting signs of primary} ovarian carcinoma

G. C. Ettorre (Presenter), G. Angone, D. Brindicci, A.P. Garribba, F. Grimaldi; Bari

CT specific findings in peritoneal spread of some tumors are widely described. The aim of this investigation was to analyze two aspects of peritoneal metastasis calcifications inside metastatic nodule and peritoneal pseudomixoma.

120 abdominal contrast enhanced CT scans, obtained in patients affected by peritoneal carcinomatosis, retrospectively reviewed in order to detect calcified peritoneal pseudomixoma. CT showed calcifications in $12(10 \%)$ and peritoneal pseudomixoma in 5 cases $(4.10 \%)$.

Calcified metastases were noted in right paracolic gutter, near the spleen, liver, diaphragm, in the hepatorenal fossa and on the anterior abdominal wall and sigmoid colon. In peritoneal pseudomixoma CT showed huge cystic masses, filling peritoneal cavity, metastatic nodules. Calcified metastases in 10 cases of serious ovarian cystoadenocarcinoma and peritoneal pseudomixoma in 3 cases of mucinous ovarian carcinoma were found.

In conclusion, we believe that calcified metastasis and peritoneal pseudomixoma identified by $\mathrm{CT}$, may suggest diagnosis of primary ovarian carcinoma.

\section{SPACE 1451}

Percutaneous aspiration and catheter drainage of pyogenic liver abscesses guided by computer tomography

Tibor Fazekas (Presenter), F. I. Todua, József Bátorfi, András Bálint, Miklós Máté, Mihály Ihász; Budapest

33 adult patients admitted to 3rd Surg. Dep. of Semmelweis Univ. (Budapest) and to A. V. Vishnevsky Institute of Surg. (Moscow), between January 1985 and November 1990. CT-guided aspirations and drainages of bacterial liver abscesses in 26 patients accomplished definitive sanitation $(26 / 33=78,8 \%)$. In 7 cases surgical revision was required. Percutaneous drainage of abscesses was able to achieve a temporal improvement in these cases and therefore was an effective pre-surgical measure. Postdrainage complications were in 4 patients $(4 / 33=12.1 \%)$. 1 patient died $(1 / 33=3 \%) .24$ patients had solitary and 9 ones had multiple abscesses. Treatment involves antibiotics together with drainage or puncture.

We conclude that percutaneous aspiration and catheter drainage is an effective method for treatment of pyogenic liver abscesses.

\section{SPACE 1452}

\section{Differential diagnosis of mucocele of the appendlx} J. Fernández Sánchez (Presenter), W. Bücklein, E. Klotz; Augsburg

Mucocele of the appendix (MA) is uncommon. Its differential diagnosis is broad and includes intraperitoneal and extraperitoneal lesions. Sonography (US) and CT imaging can suggest in many cases a MA and differentiate this entity from other cystic masses. However, sometimes it is not possible. The purpose of this study was to evaluate and describe the US, CT and conventional radiological appearance of MA and other liquid space-occupying lesions of the right iliac fossa, especially in cases of difficult differentiation beween these lesions. Our report includes 20 patients: 3 cases of MA and 17 cases of other cystic masses in the right middle and lower abdominal quadrant, such as mesenteric cysts, lymphoceles, benign ovarian cysts, malignant ovarian tumors (cystadenocarcinoma, malign cystic teratoma), abscesses, malignant coecum tumor, psoas pancreatic pseudocyst and hematomas. In all these cases the lesions showed similar US and CT features, so that the definitive diagnosis only by means of US/CT puncture or drainage and/or intraoperative could be established. In this scientific exhibit these cases are presented and discussed.

\section{SPACE 1453}

Computed radiography by stimulable phospor image plate for pancreatic biliary examinations

E. Fraile (Presenter), D. Jimenez, E. Cruz, J. Escribano, J. L. Cardenas, F. Aguado, J. Martin, V. Benita; Madrid

1. The purpose of our work is to evaluate the images obtained by computed radiography $(\mathrm{CR})$, and their comparison to conventional radiographies in patients with pancreatic-biliary pathology.
2. We studied thirty patients and evaluated each of them on hard copy, high resolution monitor and conventional radiographies. The images were assessed by three well trained radiologists. The identification of biliary tree and/or pancreatic duct was made by endoscopy retrograde cholangio-pancreatography (ERCP), percutaneous cholecystomy or transhepatical cholangiography (TCP).

3. Our results show a quite good correlation between the digital images and the conventionals. The degree of diagnostic accuracy is high and comparable in both of them. However, the post-processing facility of the digital radiography improves image quality, affords greater flexibility in image display, yields always consistent contrast on images, avoids incorrect exposure repetitions, reduces exploration time.

4. Computed radiography seems to be a good alternative to conventional radiography for pancreatic-biliary examinations. Permanent archiving of images and their retrieval and reevaluation possibility is really useful. The digital system also conveys a remarcable lowering of the radiation dose. This digital studies are very well accepted by the clinicians.

\section{SPACE 1454}

Radiologic imaging of focal, splenic lesions with pathologic correlation T. Franquet (Presenter), R. Cozcolluela, L. Inaraja, M. Montes; Pamplona

Splenic disease (uni or multifocal) are usually associated with generalized symptoms or when another examination discloses splenomegaly or a mass in the left upper abdomen. In this exhibit the imaging findings (plain films, sonography, CT and angiography) in cystic, tumoral, inflammatory and focal vascular conditions of the spleen are described and differential diagnostic criteria are reported. Several different pathologic entities of focal splenic masses will be presented along with pathologic (gross or microscopic) findings. All cases are pathologically proved and include false (secondary) cysts, epithelial (epidermoid) cysts, hydatid cysts with and without associated complications such as fistulization and abscessification, abscesses, tuberculosis, nodular sarcoidosis, inflammatory pseudotumor, hemangioma, metastases, hematomas, infarction, aneurysm and splenic peliosis.

\section{SPACE 1455}

Angiographic aspects of diffuse parenchymatous desease of the liver with dynamic $\mathbf{C T}$

J. M. Friedrich (Presenter), P. Schnarkowski, A. Goldmann, A. Jentschura; UIm

Morphology and distribution of the main intrahepatic vessels are well known and are corresponding to definite anatomical standards. These parameters show modifications in diffuse parenchymatous desease.

Therefore we studied 150 patients with dynamic CT. 50 patients had histologically proved liver cirrhosis, 50 patients presented with liver steatosis, 50 patients with normal liver were scanned routinely. A cross section was defined at the level of the hepatic bifurcation. Criteria to evaluate the anatomic feature included the total number of vessels, the number of portal vein branches, the diameter of the vessels. Vessels with a diameter of more than $8 \mathrm{~mm}$ were observed mostly in normal liver and steatosis ( $p 0.01$ ). In cirrhosis the number of vessels larger than $8 \mathrm{~mm}$ in diameter was significantly reduced $(p 0.01)$ Branches of the portal vein were also significantly reduced in patients with cirrhosis. In all patients with normal liver the hepatic vein could be opacified, but only in $33 \%$ of patients with cirrhosis. Although the diagnosis of hepatic cirrhosis is not primarily established by CT, these findings may be helpful in the evaluation of hepatic diseases.

\section{SPACE 1456}

Peritoneography in the pre-operative diagnosis of inguinal hernia V. Garcia-Medina (Presenter), J. D. Berna, E. Capitán, J. Llerena, J. Guirao J. Garcia-Medina, M. Madrigal, J. L. Genovés; Murcia

In this exhibit we evaluated the role of peritoneography in the preoperative diagnosis of unsuspected contralateral inguinal hernias among patients with clinical diagnosis of unilateral hernias. $M$ ad $M: 64$ patients with clinically detected unilateral inguinal hernia (55 right, 9 left) underwent preoperative peritoneography using $40 \mathrm{cc}$ of non-ionic contrast injected into the peritoneum in the left lower quadrant without complication.

Results: Peritoneography confirmed unilaterial inguinal hernia in all 64 patients with positive physical examination. 20/64 (31.2\%) patients had contralateral hernias diagnosed by peritoneography all confirmed at surgery. A higher percentage of contralateral hernia was found among patients with palpable left inguinal hernia $(6 / 9: 67 \%)$ than among patients with palpable right hernia $(14 / 55: 25 \%)$. 


\section{SPACE 1457}

Resection and colo-anal anastomosis with and without colonic reservoir for rectal carcinoma and large villous adenoma: radiological aspects

Maria Grazia Genovese (Presenter), Mario Delle Piane, Renato Bocchini, GianPaolo Cavallotti, Luigi Locatelli; Torino

Long term survival and local recurrence rates after anterior resection are similar to those obtained by excision when the length of rectum "laudable" removed below the tumor is $2 \mathrm{~cm}$ or more. In this cases the whole of the rectal mucosa can be treated without an abdominal perineal resection by means of a sutured anastomosis between the colon and the anal canal.

We study 18 patients; in 6 cases the rectal lesion was a large villous adenoma and in 12 cases was an adenocarcinoma of the middle third (distance from the anal margin $\mathrm{cm} \mathrm{6-7).} \mathrm{The} \mathrm{rectum} \mathrm{was} \mathrm{divided} \mathrm{and} \mathrm{transected} \mathrm{at} \mathrm{ano-rectal}$ junction level, after peranal mucosectomy two types of colo-anal anastomoses were then constructed: simple end to end ( 9 cases) and side to end after construction of a colonic $\mathrm{J}$ reservoir ( 9 cases).

Contrast X-ray, in AP, OAS, OAD and L-L projection, at small and great filling, is of great value in neo-rectum morphological and functional evaluation. It is possible to quantize the continence degree: alvus rate is in fact, proportional at rectal motor length.

It was possible to show a perianastomotic fistula that deferred transient transverse colostomy.

\section{SPACE 1458}

\section{Evaluation of pancreatic transplants with duplex Doppler US}

R. Gilabert (Presenter), C. Bru, M. J. Ricart, L. Fernandez-Cruz; Barcelona

From February 1986 to November 1990 we have prospectively studied with duplex Doppler US (DD) 25 insulin dependent type I diabetic patients who underwent whole pancreas transplantation ( 27 grafts) with pancreaticocystostomy. The aim of the study was to determine the utility of DD in: 1 . diagnosis of vascular thrombosis, 2. diagnosis of rejection. Pulsed Doppler (PD) study was performed at hilar/parenchymal level and PD arterial spectrum was quantified by the resistive index (RI). Pancreatic size, echostructure and perigraft fluid were evaluated by real time US (US). DD findings were correlated with clinical/biochemical data and pathologic studies in 9 cases.

Normal functional grafts showed a homogeneous echostructure and low resistance arterial PD spectrum (mean RI $0.60 \pm 0.15$ ). Venous thrombosis was diagnosed by PD in 6 grafts disclosing absent venous flow in 6 , and increased RI in $5(R I=1$ with reversal diastolic blood flow). Fifteen out of 18 grafts rejection episodes showed an increase of RI $(0.86 \pm 0.11)$. US findings (increased graft size, heterogeneous structure, perigraft fluid) could not differentiate graft rejection from venous thrombosis.

Conclusions: 1. PD allows a non-invasive diagnosis of venous thrombosis, 2. Correlation of biochemical data with RI $(0.8)$ points to the diagnosis of rejection.

\section{SPACE 1459}

Colon enema with contrast agents in MRI staging of rectal cancer A. Giovagnoni (Presenter), P.Ercolani, M. Misericordia, E. De Nigris, F. Amici; Torrette di Ancona

Purpose: The purpose of this study is to define the diagnostic utility of contrast agents colon enema in MRI staging of rectal neoplasms.

Methods: 42 patients $(30 \mathrm{M}, 12 \mathrm{~F})$ with proved rectal neoplasms by traditional methods (BE, Endoscopy, US, CT) underwent MRI investigation using a superconductive 1.0 T (Magnetom) tomograph unit. All patients were studied with MRI before and after retrograde recto-sigmoid filling by $300 \mathrm{ml}$ of paramagnetic agent: 20 patients using a Fe-gluconate solution (JKAI) (group I); 14 patients using a Gd-DTPA aqueous solution ( $1 \mathrm{mM}$ ) (group II); 8 patients using air as contrast agent (group III). Multiplanar, SE TI and T2 weighted images were obtained. MRI findings before and after contrast agent administration, were evaluated undependently by two observers. MRI findings were correlated with surgical or pathologic data.

Results: Colon enema with contrast agents provide a better identification of parietal lesions in 16 patients of group I, 10 patients of group II and 4 patients of group III. The lesion's detection appear the most important parameter for a correct MRI staging of rectal cancer.

Conclusions: The results show a general improvement of MRI diagnostic efficacy by use of intraluminal contrast agent; therefore the contrast media should choose in relation to clinical situation.
SPACE 1460

\section{Imaging of rare benign focal hepatic lesions}

M. Giudice (Presenter), E. Andorno, T.Avataneo, S. Cirillo, D. Regge, L. Garretti; Torino

The purpose of this study is to describe the radiological aspect of rare benign focal hepatic lesions and to suggest which imaging technique is most adequate for their diagnosis.

We have included in our series 27 patients with surgically $(67 \%)$ and biopsy (33\%) proven diagnosis. Patients underwent US, CT, MR and in $44 \%$ of cases liver angiography.

In 10 patients i.v. infusion of cholangiographic contrast material was performed if doubt persisted in differential diagnosis between hepatic adenoma and focal nodular hyperplasia.

Twenty-one patients were females and 6 males with the following rare focal lesions: adenomas $(59 \%)$, focal nodular hyperplasia $(22 \%)$, tubercolomas $(11 \%)$, haemorrhagic cysts and biliary cystoadenomas $(4 \%)$.

The typical aspect and abnormal patterns of lesions are reviewed with all digital imaging techniques available.

\section{SPACE 1461}

Rectal stenosis: dilatation with Gruntzig balloon

D. Gomez Santos (Presenter), G. B. Garcia Castaño, J. Davila Rodriguez, R. Wert Ortega, A. Glez Agudo, S. Fernandez Zapardiel, S. Oliete Sempere; Madrid

Purpose: The purpose of the panel is to show this alternative technique to surgery in the treatment of rectal stenosis of any etiology.

Methods and Materials: We show a postsurgery rectal stenosis treated with pneumatic balloon dilatation. We emphasize the technique, the radiological follow up and the results after its application.

Results: We present the results and a brief comment about the advantages of this procedure versus surgical treatment.

\section{SPACE 1462}

Leiomyomatous tumors of the small intestine: radiologic appearances on enteroclysis with pathologic correlation

N. Gourtsoyiannis (Presenter), D. Bays, M. Malamas, N. Liasis, G. Barouxis, J. Theotokas; Iraklion

Purpose: To evaluate the efficacy of enteroclysis in diagnosing leiomyomatous tumors of the small intestine, preoperatively.

Methods and Materials: The medical records, radiologic appearances and pathologic findings in 7 patients with histologically proven leiomyomatous tumors, amongst 760 referred to us for an enteroclysis in a 5-year period, were reviewed.

Results: There were six leiomyomas and one leiomyosarcoma. Gastrointestinal bleeding were the presenting symptoms in 6 and partial small bowel obstruction in one. The time interval between onset of symptoms and radiologic diagnosis ranged 3 months to five years. The jejunum was involved in one patient and the ileum in six. The type of growth was intraluminal in two cases, extraluminal in four and bidirectional in one. Radiological features for leiomyomas included: a) round or semilunar intraluminal filling defect with encroachment on the lumen or intussusception, b) tending deformity, displacement and/or identation of the intestinal wall with effacement of the overlying mucosa or ulceration and c) mass effect on neighbouring loops. Leiomyosarcoma featured as a largely ulcerated exoenteric mass. Preoperative radiologic diagnosis was correct in five and suggestive in two patients. The morphological changes of the resected specimens resembled closely the appearances shown with enteroclysis in all cases.

\section{SPACE 1463}

Arterial chemoembolization of liver tumors with liposoluble chemotherapeutic drug

Anatoly M. Granov (Presenter), Pavel G. Tarazov, Dmitry A. Granov; Leningrad

Percutaneous transcatheter arterial chemoembolization was performed in 20 patients with primary and metastatic liver neoplasms. Liposoluble chemotherapeutic drug "DX" dissolved in iodized oil was introduced superselectively in the hepatic artery for one to five times. "DX" mixed with iodized oil formed more homogeneous mass if compared with widely used oily emulsions of watersoluble cytostatics.

Retention of "DX"-iodized oil within the tumor was seen during a 20 days period postsembolization. Pathologic investigation showed $60 \%$ to $90 \%$ aseptic necrosis and fibrosis of tumor tissue. Most patients are well and asymptomatic up to 56 months after the treatment.

"DX"-iodized oil chemoembolization can be used for treatment of liver neoplasms. 


\section{SPACE 1464}

Role of ultrafast CT (UFCT) in detection and evaluation of gastro-intestinal diseases

C. Grataloup (Presenter), J.C. Boudeville, A. Hernigou, M.H. Bihet, D. Chossiere, J. C. Gaux, M. C. Plainfosse; Paris

Purpose: Tumoral, inflammatory or pseudotumoral diseases of the gastrointestinal tract explored by UFCT are illustrated.

Methods and Materials: UFCT with short exposure times ( 300 to $400 \mathrm{~ms}$ ) avoids motion artifacts and allows high resolution examinations of the digestive tract. The 43 patients evaluated include tumors (in 21 cases) inflammatory diseases (in 18 cases) and pseudotumors (in 4 cases).

Results: All localisations are reported: large bowel (15 cases) small bowel (12 cases) duodenum ( 10 cases) gastric ( 6 cases). Tumors and thickening of wall or villosities are well defined even without oral contrast because highly enhanced by intravenous contrast material. Mesenteric infiltration and lymphadenopathy are easily detected.

Conclusions: UFCT improves image quality, especially for bowel which has independent motion. The rapid acquisition capability allows the obtaining of multiple contiguous scans of the whole abdomen during the same bolus. It increases the possibilities of detection of gastrointestinal diseases.

\section{SPACE 1465}

\section{CT in lesions of the abdominal wall}

E. Grivé (Presenter), J. A. Palmer, J. Alvarez, P. Vicente, L. Gifre, L. Faigon; Barcelona

Lesions of the abdominal wall are usually identified by physical examination. The problem in these cases is to determine the nature, origin and extension of these lesions. CT is a good method for evaluating it.

To determine the computed tomographic characteristics the CT scans of 60 patients with abdominal wall lesions were reviewed. Hematomas were found in 19 patients, abscesses in 15, neoplasms in 11 , hernias in 8 , intestinal fistulas in 6 and hydatid cysts in I patient.

In all the cases CT localized the pathology and allowed an evaluation of the extent of the disease and involvement of adjacent structures. Features found to be more characteristic included a hyperdensity of acute hematoma, low-attenuation central zone of abscess with peripheral enhancement after intravenous contrast administration and herniation of air-containing bowel.

\section{SPACE 1466}

Effectiveness of transcatheter chemoembolization in hepatocellular carcinoma

Maurizio Grosso (Presenter), Francesco Spalluto, Giovanni Carlo Anselmetti, Simona Capello, Cesare Fava; Torino

Purpose: Transcatheter chemoembolization (TCE), natural history (NH) and surgical therapy (ST) of hepatocellular carcinoma (HCC) are compared.

Methods: Between December 1988 and December 199070 patients with HCC were treated. Patients have been divided in the 3 classes of Okuda; 26 patients of class $1^{\circ}$ and 44 of class $2^{\circ}$ were studied.

We employed the femoral access, according to Seldinger's method, and we catheterized hepatic artery where an emulsion of Doxorubicin and Lipiodol, followed by fibrine sponge, was injected.

Results: We evalued survival percentuals (follow-up between 3 and 24 months) at 3,6 and 12 months comparing them with (NH) and (ST) of HCC (as reported by Okuda 1985$)$. In the $1^{\circ}$ class we had $100 \%$ survival rate at 3 months $(78 \%$ in $\mathrm{NH} ; 87 \%$ in ST), $85 \%$ at 6 months (60\% in $\mathrm{NH} ; 78 \%$ in ST) and $53 \%$ at 12 months $\left(35 \%\right.$ in $\mathrm{NH} ; 70 \%$ in ST). The survival percentuals of patients in the $2^{\circ}$ class were $60 \%$ at 3 months ( $50 \%$ in NH; $80 \%$ in ST), $48 \%$ at 6 months $(10 \%$ in $\mathrm{NH} ; 65 \%$ in ST) and $20 \%$ at 12 months ( $5 \%$ in $\mathrm{NH} ; 50 \%$ in ST).

Conclusions: Surgery remains first choice treatment in HCC. TCE must be performed in patients with unresectable hepatoma as it gives a better survival rate if compared with $\mathrm{NH}$, few complications and, as our experience revealed in some cases, a reduction of HCC volume allowing radical surgery.

\section{SPACE $\underline{1467}$}

Dependence of the ultrasound B-scan on the composition and thickness of the abdominal wall: a phantom study

Uwe Haberkorn (Presenter), Günter Layer, Volker Rudat, Ivan Zuna, Adolf Lorenz, Gerhard van Kaick; Heidelberg

The influence of different examination conditions on the ultrasound B-scan was investigated using a tissue mimicking phantom and a computerized ultrasound system. Several fat-muscle-combinations were used to simulate different patients with varying thickness and composition of the abdominal wall. The ultrasound B-scans were then analyzed using mathematical statistical parameters usually applied in ultrasound tissue characterization. Thereby the influence of the abdominal wall thickness and composition on the ultrasound image brightness, microtexture and macrotexture (image sharpness) was studied. An increasing fat path caused a decrease in ultrasound image brightness and microtexture. The macrotexture was mainly unaffected. Muscle tissue had a weak influence on the microtexture parameters. Our results show that an increase in the fat layer of the abdominal wall causes a darker and unsharp image and may lead to false diagnoses especially in adiposity.

\section{SPACE 1468}

Abdominal MR imaging: barium-kaolin suspensions as negative gastrointestinal contrast agents

Andreas Heuck (Presenter), Arnulf Breinbauer, Josef Gmeinwieser; Munich

Purpose: To develop and evaluate a negative GI contrast agent for MRI based on readily available and safe compounds.

Methods and Materials: In-vitro Studies: Studies were performed at 0.5 and 1.5 T using T1- and T2-weighted spin-echo (SE) sequences (T1: TR 300-600/TE 15-30: T2: TR 2000/TE 50-400). Samples of barium-kaolin suspensions (BKS) in concentrations from $10-30 \% \mathrm{wt} / \mathrm{wt}$ barium added with $100-200 \mathrm{~g} / \mathrm{I}$ kaolin were imaged and signal intensity (SI) reduction of samples relative to a water reference was determined. In-vivo Studies: 20 volunteers were imaged at $1.5 \mathrm{~T}$ using $\mathrm{T} 1$ - and T2-weighted SE sequences $(600 / 20,2100 / 50 / 100)$. After abdominal pre-contrast imaging volunteers drank 11 of $30 \%$ barium sulfate added with $200 \mathrm{~g} / \mathrm{I}$ kaolin. A first post-contrast scan was performed $5 \mathrm{~min}$. p.c., repeat exams every $30 \mathrm{~min}$. over $2-4 \mathrm{~h}$. Peristalsis was reduced by Buscopan ${ }^{\oplus}$ i.v. Contrast effects, distribution, safety and tolerance were evaluated.

Results: In-vitro Studies: At $1.5 \mathrm{~T}$ BKS yielded a maximum SI reduction compared to water of $81 \%$ and $98 \%$ in $\mathrm{Tl}$ - and $\mathrm{T} 2$-weighted sequences, respectively. At $0.5 \mathrm{~T}$ the maximum SI changes resulted in signal increase up to $110 \%$ in $\mathrm{T} 1$ - and a SI decrease of $97 \%$ in T2-sequences. In-vivo Studies: With $30 \%$ BKS the whole Gl tract showed strong and homogeneous negative contrast, and even distribution throughout the GI tract; it was well tolerated and showed no side-effects.

Conclusions: BKS hold high potential as negative GI contrast agents for MR imaging at $1.5 \mathrm{~T}$.

\section{SPACE 1469}

\section{The automatic biopsy gun}

Kenneth D. Hopper (Presenter), Dean E. Baird, Steve H. Parker, John F. Cardella; Hershey, PA

Purpose: This study compares the efficacy of 5 types of biopsy guns versus 10 other commonly used biopsy needles.

Methods and Materials: We performed open hepatic and renal biopsies in 15 pigmy pigs. The specimens were evaluated separately in a double-blind fashion by two histopathologists using graded criteria.

Results: Significantly better tissue was obtained with a 14 gauge Tru-cut needle. Of the 18 gauge biopsy needles, the best tissue was obtained with the long-throw Bard Biopty gun, the Franseen needle, and the Cook biopsy gun. The cores obtained with the 18 gauge Chiba and 17 gauge Jamshidi needles were also above average, and all five of these biopsy needles yielded adequate tissue $80 \%$ of the time or better. The short-throw 18 gauge Bard Biopty gun, the 14 and 18 gauge Klear-Kut gun, the Cook and Turner 18 gauge biopsy needles, the 14 gauge Vim Silverman needle, and the 18 gauge Cook Vacuum biopsy needle yielded adequate tissue in less than $67 \%$ of the biopsies. The long-throw Bard Biopty gun appears the best of the automated biopsy systems. However, the 14 gauge Truc-cut biopsy needle consistantly provides an excellent histopathological specimen for diagnosis.

\section{SPACE 1470}

\section{Evaluation of the pancreas by MR imaging}

Yoshimasa Imanishi (Presenter), Vivian Y. Hou, Alexander C. Chako, Elias A. Zerhouni, Clare M. C. Tempany; Kawasaki

MR imaging criteria for the normal pancreas were established and compared with MR images of patients with pancreatic diseases. Eighty-nine patients without pancreatic disease served as controls. Pancreatic disease cases included 23 pancreatic carcinomas with or without pancreatitis, 4 cholangiocarcinomas invading the pancreas, 4 extrapancreatic masses mimicking primary pancreas neoplasms, and 9 cases of pancreatitits. Axial Tl-weighted $(T R=500-600: \quad T E=20)$, Proton- and T2-weighted $(T R=2500-2700$ $\mathrm{TE}=30 / 80$ ) images using spin-echo sequences were obtained on a $1.5 \mathrm{~T}$ system. On $\mathrm{Tl}$-weighted images, imaging criteria were developed from measurements of pancreas size in eight sites. On T1-, Proton-, and T2-weighted images, border definition, and signal intensity of the pancreas were compared with that of renal cortex, spleen, and fat to develop imaging criteria. Using this criteria, 21 of 40 pancreatic disease cases showed an abnormality of pancreas size. Total or partial obliteration of the pancreatic border was seen in 18 of 22 patients with evidence of pancreatitis on T2-weighted images. Signal intensities in tumor and pancreas were abnormal in 23 and 20 , respectively, of the 40 . All 
4 extrapancreatic masses were differentiated from intrapancreatic tumors by T2-weighted images. MRI is useful in detecting abnormality of the pancreas.

\section{SPACE 1471}

Acute gynecologic pain: diagnosis by the radiologist-sonologist A. Avila R., J. Jiménez G. (Presenter), E. Añorbe M., S. Borruel N., J.M. Cantarero, J. Manrique C.; Madrid

Purpose: We present our sonographic experience as radiologists-sonologists in the diagnosis of acute gynecologic diseases.

Methods: A total of 49 abnormal sonographies were reviewed. Surgery was performed in 35 cases; the other 14 cases were resolved spontaneously. Our cases include: 23 ectopic pregnancies (E.P.), 8 pelvic inflammatory diseases (P.I.D.), 7 haemorrhagic ovarian cysts, 2 endometriomas, 1 ovarian torsion and 1 hematometra caused by an imperforate hymen. Conventional equipment (without transvaginal transducer) was always used. The sonographic signs were analyzed and integrated with clinic and biochemical findings.

Results: Important signs in the diagnosis were: extrauterine gestational sac in E.P.; adnexal masses with septations, intracystic debris and/or high trough transmission in haemorrhagic masses or in P.I.D. The sensibility in E.P. was $100 \%$, specificity $80 \%$, positive predictive value $88 \%$, negative predictive value $100 \%$ and accuracy $92 \%$.

Conclusions: Sonography with conventional equipment has been very useful in the diagnosis of acute gynecologic pain. The knowledge of the clinic and biochemical findings is essential to the correct interpretation of the sonographic signs. So that, in daily practice, the radiologist-sonologist has to play a more active role in the diagnosis of these diseases without need a non radiologist-sonologist.

\section{SPACE 1472}

Preoperative staging of rectal carcinoma by intrarectal sonography Frank B. M. Joosten (Presenter), Jelle O. Barentsz, Simon P. Strijk, Theo Wobbes, Harry J. M. Joosten, Gerd Rosenbusch; Nijmegen

Purpose: Evaluation of intrarectal sonography in comparison to histopathological findings in patients with rectal carcinoma.

Patients and methods: 54 patients with rectal carcinoma accessible for intrarectal sonography were examined to stage local tumor extension. Sonographic results were compared to histopathologic findings of surgical specimens. In this study a radial $7 \mathrm{MHz}$ and a linear array $5 \mathrm{MHz}$ transducer were used. Results: In this group of 54 patients intrarectal sonography proved to be accurate in 48 of 54 cases (accuracy $88 \%$ ). The sensitivity for invasion of the perirectal fat was $94 \%$, the specificity was $78 \%$. The false negative results were influenced by transducer related artifacts and the experience of the radiologist. Two patients were understaged, four patients were overstaged. Patient selection and experience of the radiologist are necessary to reach high accuracy. Perirectal pathologic lymphnodes could only be detected in a minority of cases $(8)$ and the sensitivity was low $(53 \%)$.

Conclusions: Intrarectal sonography can give accurate preoperative information about extension of rectal carcinoma but is of limited value in detection of perirectal lymphnode metastases.

\section{SPACE 1473}

Digital fluorography in the examination of the allmentary tract C. Bosio, E. Juliani (Presenter), G. Margarita, R. Zatteri; Torino

This study has been performed employing Philips Digital Spot Imaging (DSI), connected to a remote-controlled table.

The digital image processing provides high resolution images at programmed acquisition speed (up to $8 \mathrm{i} / \mathrm{s}$ ), on a matrix of $512^{2} / 1020^{2}$.

Digital fluorography allows both morphological and dynamic studies, such as deglutition (even in paediatric age), or gastric pouch emptying both in gastrectomized patients and after Mason's gastroplasty for severe obesity.

Double contrast small bowel enteroclysis has been improved by the employement of DSI; truly comprehension of functional disorders occurring in malabsorption, the emptying of surgical stumps, as well as surgical anastomoses` wideness has gained from dynamic studies.

Some representative examples of alimentary tract disorders are presented.

\section{SPACE 1474}

Intraarterial application of cytostatic drugs with microembolization in the abdominal region

M. Kašpar jr., M. Kašpar (Presenter), J. Drechslerová, J. Koláŕ ; Prague

We carry out the intraarterial application of cytostatic drugs in inoperable tumours of the liver, gallbladder, kidneys, colorectal carcinoma and the metastatic processes of the liver. We try to reach the decrease of tumours as far as is possible to be operated, and so to improve the last time of patient's life. The catheter is inserted via Seldinger method into the nutrient artery of the tumour. We apply cytostatic drugs in form of the intraarterial slowly dripping infusion and partly in repeated injections. In most of the cases we try to carry out a microembolization with microcellulose which functions as the obliteration of the small arteriolas of a diameter 5-100 microns. We link cytostatic drug on the microcellulose and so we prolong the time of its effect in the pathological tumorous bed and partly the cytostatic effect is potentiated with induced ischemia. As far as the operation is not possible we repeated this way of treatment in intervals of 6-8 weeks. With this therapy we treated 115 patients with colorectal carcinomas, 36 patients with tumours of kidney, 10 with hepatomas, 40 with metastatic tumours of liver, 7 with tumours of gallbladder and 15 with cancer of pancreas. The best results we reached with the treatment of colorectal cancer, the average time of survival of operated patients were 5 years, not operated 3 years.

\section{SPACE 1475}

The computed tomographic (C.T.) appearances of choledochal cyst (Todani classification $(-V)$ and their diagnostic value Shahid Khan (Presenter), Nigel Raby, John Karani, Edward Howard; London

Introduction and Methods: Choledochal cysts are rare with some types being extremely rare. Several case reports have appeared of C.T. findings of choledochal cysts. These cysts can however have widely different appearances according to the type of cyst (Todani types I-V). This is the first attempt to illustrate the different types and describe their appearances.

Results and Conclusions: The C.T. appearances have been illustrated and have been shown to be a useful non-invasive diagnostic tool. Classification of the cysts into intra hepatic/extra hepatic or both is of value to the surgeon pre-operatively.

C.T. may also obviate the need for invasive procedures such as percutaneous transhepatic cholangiography or endoscopic retrograde cholangio pancreatography when Ultrasound is not diagnostic.

\section{SPACE 1476}

Unusual causes of cystic lesions of the liver seen on computed tomography (C.T.)

Shahid Khan (Presenter), Nigel Raby; London

Purpose: To show the Computed Tomography appearances of cystic liver lesions and to highlight the similarities and differences in patients with many different diagnoses.

Methods: A review of all cystic lesions seen on C.T. scanning over a 3 year period was undertaken. This included true cysts, false cysts and lesions mimicking intrahepatic cysts. All the diagnoses were confirmed by either serology, cyst asperation/biopsy, operative findings or post-mortem findings. Results: The differential diagnosis is much wider than usually considered. There is a large overlap in the C.T. appearances in most cases, although some conditions have features highly suggestive of the diagnosis. Some of the more unusual causes have been highlighted with illustrations, i.e. sub-capsular spread of hydatid cysts, mesenchymal hamartomas, cholangiocarcinoma, lymphoma, choledochal cysts, carcinoid tumours, and cystic haematoma. Conclusions: It is unwise to assume cystic lesions of the liver are simple cysts on the C.T. appearances alone. Only rarely are the appearances highly suggestive of a diagnosis.

\section{SPACE 1477}

Casuistic radiological findings in the female genitalia in condition of translucent hysterosalpingography - our modification Christina Kovatcheva (Presenter); Vidin

The report presents abundant material of valuable radiological findings using the translucent hysterosalpingography by means of the Schulze's device, modified by us.

The goal is to prove the advantages of this new technique of double contrast of the uterine cavity in comparison with the usual HSG.

1. Visualization of the female genitalia in congenital and acquired incompetence of the cervical os.

2. Advantageous traverse and visualization of the fallopian tubes.

3. Visualization of the pathologic changes of the uterine wall and the uterine cavity in their earliest stages - submucous fibromas, endometriosis and intrauterine carcinomas.

Speed, safety and effectiveness of this technique makes it the method of choice of many gynecologists and radiologists. 


\section{SPACE 1478}

\section{Partial splenic embolization with dextran microspheres} A. Krajina (Presenter), A. Hlava, P. Hủlek, L. Kubeš, P. Eliáš; Hradec Králové

The most feared complication of the splenic transcatheter embolization is the splenic abscess formation caused by major splenic infarction. This complication was considerably reduced by partial and stepwise embolization of the spleen. In an attempt to perform more homogeneous and safe splenic embolization, we used small $(0.05 \mathrm{~mm})$ dextran microspheres (Sephadex G 50) in humans.

Eight patients with hyperplenism were treated with dextran microspheres partial occlusion of the splenic artery. Repeated embolization was carried out in three cases. We followed a strict aseptic technique during procedure, antibiotic prophylaxis and pain control after the methodology outlined by Spigos et al., 1979.

White blood cell and platelet response to embolization marked improvement in all cases. Lowgrade fever and mild abdominal pain were present in all patients the following four days. One patient had remained febrile for twenty days. Ultrasonography examination of her embolized spleen revealed areas of hypoechogenity correlated presumably with infarcted splenic tissue.

\section{SPACE 1479}

The reliability of biochemical liver function tests in patients with ultrasonically diagnosed hepatic metastases

S. Kurbel (Presenter), B. Radosavljević, M. Dičić, I. Krnić, D. Mileusnić, O. Rubin, Z. Filaković; Osijek

The study included 205 female breast cancer patients (Bc), 54 large-bowel cancer patients $(\mathrm{LBc})$ and 54 lung cancer patients $(\mathrm{Lc})$. Serum AF, SGOT SGPT and Gamma-GT were measured together with abdominal ultrasound. Gamma-GT showed highest sensitivity, and SGOT was most specific for detecting hepatic metastases. In Bc and Lc patients SGOT showed highest overall accuracy. In LBc patients, Gamma-GT and AF were more accurate. SGPT was less specific in Bc and LBc patients. Increased numbers of true positive Gamma-GT and false negative SGPT were observed in LBc (Chi-square 22.45, $\mathrm{p}<0.01$ ).

In $21 \mathrm{Bc}, 25 \mathrm{LBc}$ and $7 \mathrm{Lc}$ patients number of hepatic lesions was multiplied with average lesion diameter as a liver involvement product (LIP) and correlated to the values of biochemical tests.

Regressions were significant only for LBc patients. AF curve $(i=0.537$, $p<0.01)$ attributed elevated values to LIP over 3 , SGOT curve $(i=0.440$, $\mathrm{p}<0.05)$ to LIP over 5 and SGPT curve $(\mathrm{i}=0.393, \mathrm{p}<0.05)$ to LIP over 10 Gamma-GT $(\mathrm{i}=0.536, \mathrm{p}<0.01)$ curve attributed pathological values to all LIP values.

Gamma-GT remains the most sensitive liver function parameter for the detection of hepatic metastases, but less specific than SGOT.

\section{SPACE 1480}

Supraclavicular lymph node metastases in carcinoma of the esophagus and gastroesophageal junction; assessment by $\mathrm{CT}$, US and US guided fine needle aspiration blopsy

H. van Overhagen, J.S. Laméris (Presenter), M.Y. Berger, A.I.J. Klooswijk, H.W. Tilanus, R. van Pel, H. E. Schütte; Rotterdam

Purpose: The preoperative assessment of supraclavicular lymph node metastases was prospectively studied in one hundred patients with carcinoma of the esophagus and gastroesophageal junction.

Methods and Materials: CT, US and palpatory findings were compared and US guided fine needle aspiration biopsy (UGFAB) was performed of nodes with a small axis of $5 \mathrm{~mm}$ or more.

Results: Supraclavicular metastases were detected on CT in $84.6 \%$ and on US in $87.5 \%$ of cases, but were palpable in only $18.7 \%$. The predictive value of a supraclavicular node indicating metastases was $0.74 \%$ on US and $0.85 \%$ on CT. Metastases were diagnosed in $21.7 \%$ of patients with squamous cell carcinoma (relative risk $=1.5$ ) and $10 \%$ of patients with adenocarcinoma. Carcinomas of the upper and middle esophagus metastasized most frequently to the supraclavicular nodes (relative risk $=1.9$ ). Metastastic nodes had a round configuration with a statistically significant greater short to long axis ratio than benign nodes $(0.89$ v.s. $0.54, p=0.05)$. In $25 \%$ of cases supraclavicular metastases were not associated with radiologically detectable mediastinal or abdominal adenopathy. Supraclavicular US or CT and UGFAB should be included to optimalise the preoperative staging of esophageal and gastroesophageal junction carcinoma.

\section{SPACE 1481}

99m-TC-HMPAO labelled leucocytes (WBC) In acute colonic diverticulitis

E. Lantto (Presenter), T. Lantto, R. Mokka, S. Ristkari, M. Vorne; Hollola

The diagnosis of acute colonic diverticulitis is based largely on clinical judgement. Barium enema and endoscopy are contraindicated in the acute stage.

Eleven patients with clinical suspicion of acute diverticulitis were imaged with labelled leucocytes to evaluate the value of this method in this disease. Images were obtained at $0.5,2$ and $\mathrm{hr}$ after reinjection of labelled cells. The results were compared with computed tomography (CT) in 9 cases and with ultrasonography (US) in 9 cases. WBC showed a focal "hot" spot in all cases with the final diagnosis of diverticulitis $(8 / 11)$ and in one colonic adenocarcinoma with chronic inflammation. Diverticulas and thickening of the colonic wall and/or pericolic oedema was seen in all cases of the disease imaged with CT (7/9) and in the colonic adenoca. CT was true-negative in one case. US was true-positive in three, false-negative in four and true-negative in two cases.

The findings were correct in $10 / 11$ cases with WBC, in $8 / 9$ with CT and in $5 / 9$ with US, respectively.

In conclusion, $99 \mathrm{~m}$-Tc-HMPAO labelled leucocytes offer a rapid, safe and accurate method in the confirmation of the diagnosis of colonic diverticulitis in the acute stage.

\section{SPACE 1482}

MRI evaluation of abdominoperineal resection for rectal cancer R. Lanza (Presenter), E. Ravasi, M. Cerioni, L. Carotti; Ancona

Authors report a prospective study comparing the findings at computed tomography (C.T) and magnetic resonance imaging (MRI) in evaluation of the pelvis in 30 patients after abdominoperineal resection for colorectal carcinoma.

MRI was performed in patients with suspected presacral recurrency on CT examination or when $\mathrm{CT}$ was unable to discriminate fibrosis from recurrent tumor.

The results were compared and verified with findings at biopsy, surgical exploration or follow-up.

By characterizing $\mathrm{Tl}$ and $\mathrm{T} 2$ values, MRI has demonstrated a superior ability to distinguish malignancy from fibrosis and both from normal soft tissue of various relocated organs. Recurrent tissues have prolonged $T 1$ and $T 2$ values, which give a low signal on $\mathrm{Tl}$ weighted images but medium-to high signal on T2 weighted images. Postoperative and radiation fibrosis consistently displays low intensity signal both in $\mathrm{T} 1$ and $\mathrm{T} 2$ weighted images.

Posteriorly relocated organs (especially uterus and seminal vesicules) can be easily recognized by high contrast and multiplanar section of MRI.

\section{SPACE 1483}

Adult presacral cystic hamartoma: US, CT and MRI evaluation F. Laurent (Presenter), G. de Verbizier, V. Lafarge, J. Faivre, M. Boisserie-Lacroix, J. Drouillard; Bordeaux

Cystic hamartoma is a rare lesion believed to originate from the embryonic tailgut that normally disappears in early fetal development. Our purpose was to describe the spectrum of US, CT and MR findings of these lesions and to emphazise the differential diagnosis with other presacral masses.

Methods: Features of imaging studies (US, CT and MRI) were retrospectively correlated with surgical and pathological findings in 10 patients. A comparison with imaging studies of more than 30 other presacral masses was performed. Results: Shape, volume, content, rectal wall involvement and coccygeal attachment, the latter representing the most suggestive findings were well demonstrated by CT. Transrectal US and MR imaging showed the precise location of the lesion originating between internal and external sphincter. Differential diagnosis included bone and soft tissue neoplasms, rectal and ovary tumors, meningocele, dermoid cysts, chordoma, abscesses and neurogenic tumors.

Conclusions: Imaging studies can suggest the diagnosis of presacral cystic hamartoma and modify the surgical strategy.

\section{SPACE 1484}

The effects of treatment of malignant liver tumors by the method of superselective arterial perfusion with oil contrast media and cytostatics Slobodan Lavrnić (Presenter), S. Radojković, D. Ljubisavljević; Belgrad

Our intention is to show that superselective arterial (transcatheteral) perfusion of the liver is a very simple, excellently tolerated, most effective and the cheapest palliative method of treatment of malignant (primary and secondary) non-resectable liver tumors.

Over the last 5 years we performed this procedure in over 200 patients and in a few of them in more than 6 sessions in 4 months interval. We have encountered 
no complications and a therapeutical response was prompt (in the first days after the procedure) with diminution of liver swelling and better general condition of the patients. The results were not favorable only in the patients who were in the terminal stadium of disease. The survival rate of our patients was similar to those published up to date.

\section{SPACE 1485}

CT-evalutation of segmental liver anatomy

Ronald Leppek (Presenter), J. Rüschoff, H.-J. Klotter, J. Freis, K.-J. Klose; Marburg

a) Liver surgery demands a preoperative determination of resectability, assessment of tolerable parenchymal injury and precise segmental localization of focal hepatic lesions. This is based on Couinaud's model of the portal and venous vascular pattern.

b) To apply CT imaging to this model, a postmortem study was performed. The portal and venous vessels of 50 complete human livers were impregnated and scanned in simulated abdominal cavity position. Consecutive sections in identical position demonstrating macroscopic anatomy were correlated with CT vessel identification.

c) Vessel orientation provided by CT imaging allows segmental identification and segmental correlation of focal hepatic lesion.

d) Preoperative CT imaging of the liver is designed to support the surgeon's choice of operation technique.

\section{SPACE 1486}

CT diagnosis of peritoneal and mesenteric diseases

J. Llauger (Presenter), C. Pérez, E. Castañer, C. Traid, Y. Pallardo, E. Sanchis; Barcelona

Peritoneal cavity is lined with an areolar membrane of mesothelial origin, the peritoneum, and contains several communicating spaces. Such spaces, usually not visualized on CT scans, can be distended by fluid.

Specific pathologic conditions affecting the peritoneum include inflammatory diseases, such tuberculous peritonitis, and tumoral diseases, primary (mesothelioma) or secondary (peritoneal carcinomatosis).

Small-bowel mesentery and transverse mesocolon are peritoneal folds that connect visceral structures with the abdominal wall. Like the peritoneum, pathologic conditions can affect the mesentery, including congenital abnormalities (mesenteric cysts), inflammatory and tumoral diseases.

CT is the method of choice in the diagnosis of peritoneal and mesenteric diseases. Representative examples of these conditions are presented and main radiological findings are described.

\section{SPACE 1487}

Liver tumors: CT-portography evaluation

J. Llauger (Presenter), C. Pérez, P. Coscojuela, C. Traid, A. Giménez, E. Sanchis, X. Alomar; Barcelona

CT and sonography have become the primary imaging methods for the detection of hepatic metastases and hepatocellular carcinomas. However, their success rate in detecting small lesions is low for planning surgery. New advances and increasing number of indications for hepatic resections have prompted the development of new methods with a greater sensibility.

In recent years, several techniques, including $C T$ with ethiodized oil emulsion (EOE-CT), delayed scanning, CT-angiography and Magnetic Resonance, have become available and are now frequently used to provide more complete information about the number of lesions, intrahepatic segmental location, and presence of portal vein thrombosis.

CT angiographic techniques include CT-angiography (CTA), performed during contrast injection into the hepatic artery and CT during arterial portography (CTAP), the most commonly used.

The purpose of this essay is to present the technique, results, and advantages of CTAP and illustrate the findings on hepatic tumors, particularly those that may be confusing. Several no reported causes of false positive or false negative CTAP scans are decribed.

\section{SPACE 1488}

Percutaneous drainage of post-operative abdominal collections: value of very large-bore catheters

P. Maquin (Presenter), C. Poey, E. Bloom, J. Giron, F. Joffre, J. J. Railhac; Toulouse

15 patients with septic post-operative abdominal collections were percutaneously drained by 24 french double-lumen catheters.

Insertion of the catheter was performed in all cases under local anaesthesia, guided by CT and fluoroscopy.

Clinical improvement was obtained immediately, along with the collapse of the abscess. Percutaneous drainage, maintained 7 to 15 days (mean: 10 days) has allowed to avoid surgery in all cases. We had no complications.

Large catheters allow a more efficient drainage, especially in case of thick debris. Thus, they may enhance the success rate and reduce the duration of percutaneous drainage.

\section{SPACE 1489}

\section{CT and MRI after liver transplantation}

B. Marincek (Presenter), R. Fehr, M. McPhillips; Zürich

Purpose: To assess the usefulness of CT and MRI in diagnosis and management after liver transplantation.

Methods: We reviewed the CT scans and MR examinations of 21 patients. The imaging studies were done between 2 days and 27 months after hepatic transplantation. MRI was performed at 1.5 T. All patients had state of the art CT examinations with iv contrast administration.

Results: In all patients a periportal and pericaval edema due to disruption of lymphatic drainage was seen. This resolves, albeit slowly. The evolution and resolution of postoperative complications such as perihepatic fluid collections $(\mathrm{N}=17)$, hepatic perfusion defects $(\mathrm{N}=4)$ and hemorrhage of the right adrenal gland $(\mathrm{N}=3)$ was documented. Several patients had biliary leaks, of which no evidence was seen on CT or MRI, confirming that these are not the primary imaging modalities for biliary pathology in hepatic transplants. Two patients received livers from child donors and another a "reduced-size" liver, i.e. only part of the donor liver was transplanted. Compensatory hepatic hypertrophy was visible as early as the seventh post-operative day.

Conclusions: Many of the complications seen after liver transplantation are self-resolving, but monitoring with CT or MRI is useful to confirm resolution or reveal the need for therapeutic intervention.

\section{SPACE 1490}

Portal vein thrombosis: correlation between Doppler sonography and fast MRI.

Carlo Martinoli (Presenter), Giuseppe Cittadini, Raffaella Conzi, Caterina Pastorino, Piero Sala, Gian Andrea Rollandi, Giampaolo Grozio, Giorgio Mallarini; Genova

Duplex-doppler (DD) and most recently color doppler imaging (CDI) have become established non-invasive imaging methods of the vascular compartments. Presently, MR is not yet considered diagnostic modality of choice to assess splancnic vessels thrombosis although being effective technique in evaluating flows. This prospective work was undertaken to investigate the sensitivity and accuracy of doppler vs. fast MRI in making diagnosis of portal thrombosis (PVT). DD and CDI was obtained respectively with Hitachi AU 450 and AU590 scanners with $3.5 \mathrm{MHz}$ convex probes. MRI was performed with a $0.5 \mathrm{~T}$ apparatus using gradient echo sequences $(70 \mathrm{~ms}$ TR; $13 \mathrm{~ms}$ TE, 70 FA) on breath-holding patients. Due to entry slice phenomena, this pulse sequence was very efficient in detecting portal flow and/or thrombosis with diagnostic resolution as far as the hepatic segmental branches. Flow within liver was assessed by axial plane. On the contrast, spleno-portal axis flow was determined by coronal sections. 12 patients with PVT (incomplete PVT in 2 cases; complete PVT in 3 cases; thrombosis of PV and its intrahepatic branches in 4 cases; portal cavernomas in 3 cases) were imaged with both techniques. Briefly, fast MRI demonstrated similar accuracy to doppler techniques in diagnosing thrombosis of the main PV but lower efficiency in detecting residual flow within incomplete PVT, canalized cavernomas and periportal collaterals.

\section{SPACE 1491}

Examination about MR signal intensity of hepato cellular carcinoma Hiromichi Matsuda (Presenter), Fumio Kotake, Kimihiko Abe, Saburo Amino; Tokyo

Purpose: It's said that MRI of H.C.C. (Hepato-Cellular Carcinoma) showed low-high signal intensity on TIWI (T1 weighted image). But, on T2WI (T2 weighted image), some report that all cases showed high signal intensity, some report that in part showed iso signal. So, this time, we studied signal intensity of H.C.C. on TIWI and T2WI, and in some cases obtained pathological findings, we compared those with signal intensity of H.C.C.

Methods: We used TOSHIBA MRT-50A ( 0.5 tesla). Pulse sequences used were spin echo (SE) 500/20 (TR/TE) for TIWI and SE 2000/80 for T2WI. Objects of this study are H.C.C. 70 cases.

Results: On TIWI, MRI showed various signal intensity of low-high signal. In particular, tumors below $2 \mathrm{~cm}$ were frequently described high signal intensity. On T2WI, most of 70 cases H.C.C. showed high signal intensity. But, we acknowledged $20 \%$ of these were described iso signal intensity. The case performed an operation was well differentiated H.C.C. showed iso signal. Conclusions: MRI of H.C.C. showed not only high signal intensity but also iso signal intensity on $\mathrm{T} 2 \mathrm{Wl}$. And we think signal intensity of H.C.C. correlates to each differentiated type. 


\section{SPACE 1492}

Unresectable liver metastases therapy: comparison of single with serial lipiodol-epirubicin transcatheter arterial embolization

Giorgio Minonzio (Presenter), Gian Piero Puricelli, Giulio Carcano, Marco Tettamanti, Fabio Baruzzi, Alberto Caresano; Varese

Purpose: To perform a study on effectiveness of transcatheter arterial embolization (TAE) with Lipiodol-Epirubicin (L-E) comparing volume variations of liver metastases after single or serial treatments.

Methods and Materials: Twenty-six patients with unresectable liver metastases from gastrointestinal tract underwent TAE with Lipiodol-Epirubicin $(20-50 \mathrm{mg})$. Gelatin sponge was not employed. Eighteen of the 26 patients underwent one treatment and were followed with CT after 1,3, and 6 months. Eight patients were submitted to 3 or more treatments ( 1 month intervals) and CT after 1, 3 and 6 months. Volume was assessed with ROI-evaluation.

Results: After single L-E TAE a mean volume decrease of $8.1 \%$ was recorded after 1 month, with an increase of $17.5 \%$ after 3 months without further therapy. In patients submitted to monthly repeated L-E TAE we observed a volume increase of $8.3 \%$ at 3 months and $2.8 \%$ after 6 months. Low dose Epirubicin did not cause major complications. With single L-E TAE, though Lipiodol can stop longer within liver metastases, an antitumoral effect was observed only during the first month. After multiple monthly repeated L-E embolizations little changes of metastatic volume were recorded up to six months. The latter method proved effective and promising for improvement of survival.

\section{SPACE 1493}

MRI of small hepatocellular carcinomas less than $3 \mathrm{~cm}$ at $1.5 \mathrm{~T}$, especially the efficacy of dynamic MRI

Takeshi Miyamoto (Presenter), Hideyuki Nakajima, Noriaki Usuki, Shigeo Saiwai, Haruyuki Fukuda, Jittoku Nishikawa, Jyouji Kawabe, Masatoshi Kudou, Syuusuke Tomita; Kobe City

The efficacy of diagnosis of small hepatocellular carcinomas (HCCs) using plain MRI and dynamic MRI was studied. Materials were a total of 73 small HCC lesions less than $3 \mathrm{~cm}$ in 60 patients. In 44 of $73 \mathrm{HCCs}$, clear dynamic MR images of HCCs were obtained. Diagnosis of the HCCs were confirmed surgically in 20 lesions, in 8 by biopsy and in 45 by various examinations including US, angiography, Dynamic CT, CT after lipiodol infusion, CT during arterial portography, US under arterial infusion of $\mathrm{CO}_{2}$ micro-bubbles, color dopplering, and clinical data. Dynamic gradient echo MR images using fast low angle shot (FLASH) technique at $90^{\circ}$ pulse angle were obtained after bolus injection of Gd-DTPA $(0.1 \mathrm{mmol} / \mathrm{kg})$ under suspended respiration. Eighty-two \% of HCCs showed high-intensity on T2-weighted images. Ninetythree $\%$ of HCCs revealed enhancement of tumors at early phases after injection of Gd-DTPA on dynamic MR images. HCCs which did not reveal enhancement were highly differentiated HCCs, which showed high-intensity on $\mathrm{Tl}$-weighted images and iso-intensity on $\mathrm{T} 2$-weighted images. Dynamic MRI further increases the qualitative value of plain MRI diagnosis. The comparison with adenomatous hyperplastic nodules was also studied.

\section{SPACE 1494}

\section{Colitis and Behçet's syndrome}

M. J. Moreno (Presenter), I. Ferreres, J. Perez Clavijo, P. Gil, J. M. Pascual, J. Primo; Sagunto

Behçet's syndrome is a multisystem inflammatory disorder, of unknown etiology, however some authors consider this entity as a vasculitis.

Diagnosis focuses on clinical findings characterized by three major criterias: Recurrent aphtous oral ulcerations, genital ulcers and ocular inflammatory involvement.

In addition Behçet's syndrome can also involve many other organs and systems: Skin, vessels, gastrointestinal tract, etc ...

Gastrointestinal tract involvement affects any location, predominating in colon and terminal ileum. Ulcers of varying size have the radiological appearance of a colitis.

Differential diagnosis of Behçet's syndrome must be carried out taking into account the possibility of inflammatory intestinal disease (Crohn's disease and ulcerative colitis).

In our study we present four patients diagnosed as having Behçet’s syndrome. Two of them developed gastrointestinal manifestations and radiologically severe pancolitis.
Comparative evaluation of $\mathrm{x}$-rays, LES manometry and $\mathrm{pH}$ testing in recurrent hiatal hernia

M. T. Bonfanti, F. Gattoni, C. Pozzato, S.Bolzoni, R. Nessi (Presenter), C. Uslenghi; Milan

The value of double contrast radiography of the esophagus, LES manometry and $\mathrm{pH}$ testing of esophageal reflux were comparatively assessed on 39 patients operated for sliding hernia. The time elapsed from surgery ranged from 2 to 9 years (mean: 5 years); in 7/39 patients clinical symptoms suggestive for disease recurrence were reported.

$X$-ray examination showed a recurrent hiatus hernia in $4 / 39$ cases $(10 \%)$. Manometry revealed a LES in supradiaphragmatic position in the same 4 patients. PH testing did never demonstrate a gastro-esophageal reflux: however, an abnormal detersion time after acid test was observed in 4 different cases. There was a remarkable agreement between $x$-rays and manometric findings. The 4 patients with an abnormal radiographic and manometric picture were reoperated and the results of both investigations were confirmed by surgery.

Double contrast examination of the esophagus and LES manometry may be considered the combined approach of choice for the diagnosis of recurrent sliding ernia and for the follow-up of operated cases. PH testing is a complementary procedure that can be useful for detecting the esophageal reflux which may be present without recurrent hiatal hernia.

\section{SPACE 1496}

Kaopectate as a negative rectal contrast agent In MR imaging of colorectal disease

Jörg Neuerburg (Presenter), Klaus Ch. Klose, Klaus Bohndorf, RolfW. Günther; Aachen

The potential of the generic Kaopectate (Upjohn) as a negative rectal contrast agent in MR imaging was investigated in the assessment of colorectal disease. Fifty MR examinations in patients with colorectal disease (colorectal carcinoma: 29 patients, inflammatory disease: 13 patients, miscellaneous disorders of the colon: 8 patients) were evaluated. All examinations were performed with $\mathrm{Tl}$ and $\mathrm{T} 2$ weighted $\mathrm{SE}$ sequences after rectal application of Kaopectate. Gd-DTPA enhanced T1 SE sequences were obtained as well.

Kaopectate revealed a signal void of the lumen of the large intestine in $\mathrm{Tl}$ and $\mathrm{T} 2$ weighted SE sequences. A sufficient contrast between bowel and nonbowel could be obtained in 47 of the 50 examinations. Intravenous administration of Gd-DTPA allowed a good discrimination of the high signal intestinal wall, malignant or inflammatory tissue and the low signal lumen of the bowels. No side effects were registrated.

Kaopectate improved diagnostic quality by providing a good negative contrast between bowel and nonbowel and by reduction of moving artifacts.

\section{SPACE 1497}

Ovarian neoplasms $=$ sonographic criteria of benign and malignant tumors (a retrospective study of 210 cases)

Thu Nguyen-Tan (Presenter), Thu-Ha Dao, Liliane Ollivier; Paris

Purpose: To compare sonographic features of benign and malignant ovarian neoplasms and to evaluate preoperative prediction of malignancy.

Methods and Materials: 98 examinations performed with $3.5-5 \mathrm{MHz}$ external probes were reviewed with respect to 9 criteria including: uni/bilaterality - size - echogenicity - wall and septa pattern - calcifications - fluid/fluid level ascites - metastases. These criteria were then used to predict benignity or malignancy of 112 histological proven ovarian neoplasms.

Results: Retrospectively, benign tumors $(n=25)$ are unilateral with anechoic or homogeneous solid pattern containing calcifications. Malignant tumors $(n=73)$ are more often bilateral with size exceeding $5 \mathrm{~cm}$ and wall/septa are thick and irregular or predominantly echoic and heterogeneous solid pattern associated with ascites and metastases. Prospectively, ultrasound failed to diagnose 7 benign tumors $(n=37)$ and 9 malignant tumors $(n=75)$.

Conclusions: With an overall accuracy of $85.7 \%$, a sensibility of $89 \%$ and a specificity of $75.6 \%$, sonographic tumor type prediction with external probes is not enough reliable to affect the surgical practice to remove all ovarian masses. But the wide use of endovaginal high frequency probes should improve sonographic accuracy and specificity.

\section{SPACE 1498}

Management of common bile duct stones using second generation extracorporeal shockwave lithotripsy

D. A. Nicholson (Presenter), D. F. Martin, D. E. F. Tweedle; Manchester

Endoscopic extraction of common duct stones (CDS) is performed in patients who have had cholecystectomy and in other patients who are 
considered a poor surgical risk. In about 10\% CDS cannot be removed, usually because the stone is too large to pass through the lower segment of the common bile duct. This paper describes our experience using a second generation lithotripter (Siemens "Lithostar") for CDS in patients in whom endoscopic extraction had failed or was not appropriate. With this machine no waterbath or general anaesthesia is required. Preliminary investigations to assess possible complications included: serum LFT's, U\&E's, FBC, serum amylase, bile culture and urinalysis. Abdominal CT was performed in the first 20 patients. 54 patients, 34-89 years old (mean 74.3 years), with common bile duct stones $(6-36 \mathrm{~mm})$ have been treated. All patients had nasobiliary catheters (52) or cholecystostomy tubes (2) in situ. Patients received 4000-6000 shocks per session over approximately 60 minutes. Seventeen patients underwent two ESWL sessions and two patients three or more sessions. Thirty-seven patients had one stone, ten had two and seven had three or more. Spontaneous clearance of fragments occurred in three patients and ducts were cleared endoscopically in a further 32 patients (total $=35$ patients $-65 \%$ ).

Fragmentation occurred in a further 9 patients but remaining fragments were large and have not yet been cleared. 10 patients stones remained unchanged. The response to lithotripsy was dependent on the size of stones, with number having little effect. ESWL was well tolerated without the development of any haematological, biochemical or CT abnormality. Second generation ESWL is a promising adjunctive treatment for CDS with a low complication rate, which compares favourably with elective surgery, when preliminary endoscopic extraction has failed.

\section{SPACE 1499}

Acquired malposition of the gallbladder: cirrhosis is not the only cause D. A. Nicholson (Presenter), D. F. Martin; Manchester

The position of the gallbladder (GB) was evaluated on 200 consecutive CT scans. 188 patients showed no significant hepatic abnormality and were used as controls to assess normal GB position. The GB angle was measured between the coronal plane and the axis of the GB. The normal range varied from $35^{\circ}-75^{\circ}\left(\right.$ mean $\left.52^{\circ}\right)$. The shortest distance of GB fundus to abdominal skin surface varied from $2-5 \mathrm{~cm}$ (means $3.2 \mathrm{~cm}$ ). Although liver substance lay between fundus and skin surface in only $55 \%$ of controls, in no normal patient did the fundus project beyond the surface of the liver.

Six of eight patients with cirrhosis, showed an abnormally positioned GB. In four, part or all of the GB projected beyond the surface of the liver. In two the GB fundus to abdominal skin distance measured more than $5 \mathrm{~cm}$. In all eight the $G B$ angle remained within normal limits. Interposition of the colon between liver and anterior abdominal wall was also noted in 4 . In two patients with lobar atrophy secondary to sclerosing cholangitis, the GB was malpositioned, although it maintained its normal position between the left and right lobes $\left(\mathrm{GB}\right.$ angles $5^{\circ}$ and $20^{\circ}$ ). Two patients with large right intrahepatic masses, showed subsequent increase of the GB angle $\left(90^{\circ}\right.$ and $\left.95^{\circ}\right)$.

These acquired malpositions of the GB due to cirrhosis, lobar atrophy and mass effect, have potential clinical implications with increased risk of inadvertent injury during percutaneous liver biopsy or interventional procedures and such procedures should be performed using image guidance.

\section{SPACE 1500}

Diagnostic imaging of ileocecal diseases

J.Palmer (Presenter), C. Pérez, J.Llauger, A. Giménez, P.Alvarado, X. Alomar: Barcelona

The ileocecal area is an abdominal region that often is the preferential site of infectious, inflammatory, neoplastic, vascular, and numerous other diseases. It is also a region whose location, other than upper digestive tract or distal colon, is not well suited for endoscopic evaluation. Imaging procedures have proved to be very important for the evaluation of such diseases.

Several techniques are available for imaging the ileocecal area, including plain films, antegrade bowel examination, small bowel enema, colon enema, double contrast enema, ultrasound, computed tomography and angiography. All have strengths and limitations, and there is some controversy regarding their role. We report the radiologic findings, using different techniques, in these diseases and assess the usefulness of these findings in stablishing diagnosis and differential diagnosis.

\section{SPACE 1501}

The diagnostic accuracy of US, CT and ERCP in the diagnosis of jaundice

K. Partanen (Presenter), P. Pasanen, P. Pikkarainen, A. Pirinen, S. Soimakallio, E. Alhava, E. Janatuinen; Kuopio

The purpose of this study was to investigate prospectively the diagnostic accuracy of ultrasound (US), computed tomography (CT) and endoscopic retrograde cholangiopancreatography $(E R C P)$ in the disctinction between extra- and intrahepatic causes of jaundice in 187 consecutive patients. The limit for the inclusion to the study was defined as serum bilirubin concen- tration $>40 \mu \mathrm{mol} / \mathrm{l}$.

Results: The sensitivity rates of US, CT and ERCP in the detection of obstruction were 63,77 and $87 \%$, respectively. The differences between all these methods were statistically significant. The specificity and the positive predictive values of all methods reached $96-99 \%$, but the negative predictive values were 37,48 and $63 \%$, respectively. The low values of sensitivity and negative predictive values could be explained by a large number of cases with choledochal stones that could not be detected by imaging methods or that had already passed spontaneously.

On the basis of this study it seemed reasonable not to construct a rigid flow chart for the workup of an jaundiced patient. A flexible use of the present investigative methods is recommended, and the optimal use of them requires a good knowledge of their advantages, limitations, risks and costs.

\section{SPACE 1502}

MRI evaluation of the liver after gallstones lithotripsy with shock waves P. Pavone (Presenter), D. Lomanto, S. Giuliani, G. Cardone, V. Speranza, R. Passariello; L'Aquila

Purpose: To verify the status of the liver parenchyma surrounding the gallbladder with MRI after shock waves lithotripsy.

Methods: 10 patients submitted to shock waves lithotripsy by means of a Dornier MPL 900 system were included in the study. The average energy was $22 \mathrm{kv}$, with a number of shock waves of 1200 to 1500 .

The MRI examination was performed within 24 hours from the treatment. A $0.5 \mathrm{~T}$ superconductive system was used. Spin echo $\mathrm{T} 1$ and $\mathrm{T} 2$ weighted sequences were acquired on the axial and sagittal planes.

Results: The MR images did not show any signal intensity alteration in all but one case.

In this female patient, weighing 45 kilos, a diffuse increase of signal intensity was seen, ill-defined at the level of the hepatic parenchyma surrounding the gallbladder fossa. The alteration was related to contusive microtrauma (edema or microhemorrhages). Control study was performed at two weeks, and no residual alteration was evident.

Conclusions: This study confirms the safety of shock waves lithotripsy treatment of gallbladder stones. Even in the positive case, the signal intensity alterations were mild, and no residual alteration was evident at follow up.

\section{SPACE 1503}

Selective enhancement of the liver by magnetic resonance arterlal portography (MRAP): studies with rapld acquisition spin echo (RASE) sequence

Paolo Pavone (Presenter), Silvia Giuliani, Paolo di Renzi, Casimiro Simonetti, Giampiero Cardone, Roberto Passariello; L'Aquila

Purpose: To evaluate organo specific enhancement of the liver in the portal phase during injection of Gd-DTPA in the superior mesenteric artery, with use of breath holding $\mathrm{Tl}$ weighted spin echo sequences.

Methods: 10 patients with hepatic malignancies undergoing angiography for different reasons were studied with MRAP.

MR images were obtained with a $0.5 \mathrm{~T}$ superconductive equipment. Breath holding RASE sequences were acquired, with TR $230 \mathrm{msec}$., TE $16 \mathrm{msec}$., 1 Nex and $128 \times 256$ matrix. 6 slices are obtained in 30 seconds.

Gd-DTPA was injected at a dose of $4 \mathrm{ml}$ of $0.5 \mathrm{mmol} / \mathrm{l}$ solution, diluted in water. The injection was started 15 seconds prior to the beginning of the pulse sequences and lasted as long as the acquisition time $(30 \mathrm{sec}$.).

Results: Strong enhancement was observed in normal liver parenchyma, with values of Signal Enhancement over Noise (SE/N) of 9.5. The liver lesions did not present significant enhancement (measured value of $\mathrm{SE} / \mathrm{N}=0.5$ ).

Distal to segmental portal vein occlusion no enhancement was observed in two cases.

Conclusions: This technique provides selective hepatic enhancement. The use of RASE sequences is favourable because of the number of slice obtained and the high $\mathrm{T} 1$ weighting.

\section{SPACE 1504}

\section{C. $T$. findings in intestinal ischemia}

C. Pérez (Presenter), J.Llauger, A.Zidan, A. Giménez, X. Alomar, J. Palmer; Barcelona

CT findings in 27 cases of surgically proved bowel ischemia are reviewed. Bowel ischemia is a common cause of acute abdominal pain. Clinical and laboratory data are unspecific and diagnosis is usually delayed, resulting in a high mortality. Radiologic diagnostic is also difficult. Barium studies and angiography are time-consuming, invasive or not specific.

CT has been used increasingly to evaluate patients with acute abdominal pain, allowing identification of bowel ischemia findings. These include bowel changes (Fluid-filled loops, thickened wall, contrast mural enhancement and intramural low attenuation zones or air) and/or vascular changes (portal or mesenteric air, mesenteric vein thrombosis and mesenteric artery occlusion). 
CT is specially useful to identify secondary ischemia changes in closed-loop obstruction (volvulus). An early diagnosis of this condition results in a very good postoperative prognosis.

\section{SPACE 1505}

Primary mesenteric tumors in adults

C. Gonzalez Hernando, R. Perez Arangüena (Presenter), I. Sanchez de las Matas Sanchez, M. Pastrana Ledesma, A. Fernandez Zubiraga, A. Bueno Horcajadas, A. Garcia Suarez; Madrid

We have tried to institute the diagnostic criterions intervening imaging methods (ultrasonography, CT, angiography) which let us establish an accurate pathological correlation among the primary mesenteric and omental tumors, differentiating two broad groups in relation with their characteristics (solid or fluid tumours). Everyone were verified by surgery and/or biopsy.

We have studied thirteen patients with cystic mesenteric tumors. Seven of them had real cysts, with revetment epithelium ( 3 serous cysts, 1 quilous cyst, 2 duplication cysts, 1 enteric cyst), three mesenteric pseudocysts which were not of pancreatic origin and three primary peritoneal echinococcus cysts. The most valuable criterions to diagnose them were established by CT and ultrasonography. They are: uni or multilocularness, wall thickness, evidence of calcium, fat density in CT and their location and relation with bowel loops.

We have found twelve patients with primary solid tumors of the mesentery: 2 liposarcomas, 2 sarcomas, 1 peritoneal mesothelioma, 2 primary mesenteric lymphoma, 2 mesenteric desmoid, 1 Castleman's syndrome, I neurofibromatosis, and 1 retractile mesenteritis. In these patients, the best diagnostic method has been $\mathrm{CT}$, with very specific findings in some entities, resulting of little value diagnostic angiography.

\section{SPACE 1506}

Duplex-Doppler in liver transplanted patients

I. Sanchez de las Matas Sanchez, R.Perez Arangüena (Presenter), C. Gonzalez Hernando, J. Minaya Bernedo, P. Fraga Rivas, J. L. Perez Picouto, M. Illanas Sanz; Madrid

Duplex-Doppler sonography was used preoperatively (70 patients) and postoperatively (55 patients with 60 transplants) in liver transplanted patients. Preoperatively, Duplex-Doppler sonography detected portal vein thrombosis in 2 patients, hepatofugal flow from the portal vein to the portosystemic derivations in 3 patients with spontaneous splenorenal shunt, and 2 patients with associate malignant hepatic neoplasms (a multicentric hepatoma and a hepatoblastoma).

Postoperatively, this technique detected hepatic arterial thrombosis in 3 patients (one false positive in a patient with a long arterial stenosis), stenosis of the hepatic artery in 4 patients and thombosis of the inferior cava vein in other 2 patients (one false positive from IVC compressed extrinsically).

Duplex-Doppler sonography was also helpful to assess biliary complications (bilomas in 4 patients which were drainaged percutaneously guided by ultrasound, and obstructive jaundice in a patient with choledocholitiasis), extrahepatic fluid collections (12 patients), hepatic hematoma (4 patients), hepatic infarction ( 2 patients), hepatic abscess (1 patient) and one tumour relapse in transplanted liver (hepatoblastoma).

We have correlated our Duplex-Doppler findings with the results obtained by other methods of diagnosis (nuclear medicine, CT and angiography). We remark the absence of significant variation in the resistance index of the hepatic artery during the rejection, the high specificity $(98 \%)$ and sensitivity $(94 \%)$ to show portal vein permeability, the high accuracy of this technique to diagnose hepatic arterial thombosis and stenosis, and the biliary complications in hepatic transplant.

\section{SPACE 1507}

\section{Inhomogeneous echo-pattern in pancreas}

M. Peter (Presenter); Debrecen

Purpose: By means of ultrasound examinations radiologists often experience and describe in the report the inhomogeneity of the echo-pattern of the pancreas.

What is the significance and value of this diagnosis concerning the condition of the patient and for the physician?

Methods and Materials: 38 patients we performed the US examination of the pancreas to determine the distribution of the intensity of the echos (histogram). 38 patients we performed ERP examination.

\begin{tabular}{llll}
\hline \multicolumn{2}{c}{ US } & & \multicolumn{2}{c}{ US } \\
negative & positive & negative & positive \\
\hline homogeneous & inhomogeneous & constant & uneven
\end{tabular}


$(18.5 \%)$.

Phase II: a progressive ileal loop enlargement was seen in $16(59.2 \%)$. While not preventing normal emptying sharp angulation at the site of anastomosis occurred in $2(7.4 \%)$ and recurrent fistula in $3(11.1 \%)$.

Overall, the stool frequency (range 1-7 movs./day), based on the X-ray study alone, could not be predicted either during Phase I or Phase II of the examinations.

\section{SPACE 1511}

Quantitative analysis of the pelvic floor function by direct coronal (DC) pelvic CT

V. Piloni (Presenter), W. Montesano, S. Baleani, A. Montesi; Ancona

Purpose: To describe a method of dynamic DC CT imaging of the pelvis with emphasis focused on the definition of four parameters as critera for normal pelvic floor physiology.

Methods and Materials: DC pelvic CT was carried out using a GE/CT Pace scanner (gantry opening: $64 \mathrm{~cm}$ ) in 15 consecutive patients, 6 males, 9 females, mean age $52.3 \mathrm{yrs}$, without functional disorders of the pelvic floor. The following protocol was used: 1 . three hours before scanning $600 \mathrm{ml}$ of oral contrast is taken ( 2 per cent solution of Gastromiro Bracco); 2 . the bladder is distended by natural urine; $3.150 \mathrm{ml}$ of air are insufflated through a rectal tube just prior of scanning; 4 . the patient seats facing away from the gantry opening and is scanned at rest and on straining: 5 . following a lateral scanogram of the pelvis $10 \mathrm{~mm}$-thick sections are obtained from the femoral head to the tip of the coccyx; 6 . technical factors are: Kv 120, Ma $130, \sec 3$, Rec fov 35 , WW 500 , $\mathrm{WL}+22$.

Results: The following parameters, related to functionality of pelvic floor, were noted to provide numerical data: a) Levator Ani Angle (LAA): mean $105^{\circ} \pm 5.00$ at rest and $125^{\circ} \pm 5.00$ on straining; b) Ano-Rectal Junction (ARJ) level $(\mathrm{mm})$ above $(+)$ or below $(-)$ the ischial tuberosities: $+25.13 \pm 1.08$ at rest and $+12.06 \pm 3.11$ on straining; c) supra and infra levator spaces area $\left(\mathrm{cm}^{2}\right)$ and percentage variation (\%) on straining: $59.76 \pm 10.92$ vs $62.45 \pm 12.12(+16.57 \%)$ and $40.68 \pm 9.85$ vs $35.18 \pm 11.53$ ( $-14.79 \%)$ respectively; d) Levator Ani Muscle (LAM) length (mm) and percent increase: $39.40 \pm 2.80$ at rest and $42.80 \pm 1.70$ on straining $(+8.6 \%)$.

\section{SPACE 1512}

Contribution of abdominal sonography in the differentiation of peritoneal carcinomatosis from radiation injury

F. R. Plante (Presenter), G. Schmutz, R. K. Chhem, B. Bui, M. Y. Jeung, A. Gangi; Sherbrooke, Québec

Purpose: To determine the contribution of US examination in the diagnosis of peritoneal carcinomatosis.

Methods: From February 1987 to March 1990, abdominal sonography was performed in 96 patients with suspected peritoneal carcinomatosis. All those patients had underwent abdominal radiotherapy for malignancy. Peritoneal carcinomatosis were confirmed by clinical follow-up and/or pathologic findings at surgery or autopsy in 53 patients $(62$ percent).

Results: Recurrence was detected by US examination in 19 patients (36 percent) on the presence of visceral metastases or adenopathies. Abundant ascites was present in 9 patients ( 17 percent). In 7 patients ( 13 percent) there were no US findings suggesting malignant recurrence. Sonographic examination detected peritoneal carcinomatosis in 18 patients (34 percent). These sonographic findings were: Fan shaped mesentery ( 4 cases) peritoneal nodules ( 5 cases), hyperechoic bowel wall (9 cases). There were 3 false positives among 33 patients with no recurrent tumor. The sensitivity of sonography was 81 percent and the specificity 90 percent with an accuracy achieved of 88 percent. Conclusions: Sonography is the procedure of choice in the follow-up of malignancy, especially in cases of suspected peritoneal carcinomatosis.

\section{SPACE 1513}

CT diagnosis of lymph node metastases in hepatocellular carcinoma F. Pombo (Presenter), R. Rodriguez, C. Gallego, J. Perez-Fontan; La Coruña

Purpose: This study was undertaken to evaluate the CT spectrum of lymph node metastases in hepatocellular carcinoma (HCC).

Methods and Materials: During the last 4 years, 102 consecutive cases of $\mathrm{HCC}$ were examinated by $\mathrm{CT}$ and histologically confirmed metastatic adsominal lymph node enlargement was detected in 9 of them.

Results: The adenopathies were located in periportal $(n=6)$, peripancreatic $(n=5)$, para-aortic $(n=5)$, superior mesenteric artery $(n=2)$, celiac $(n=2)$, peridiaphragmatic $(n=1)$, mediastinal $(n=1)$ and axillar $(n=1)$ regions. Six patients had two or more regions involved. The size of the nodes was $(15-40 \mathrm{~mm})$ in 7 cases and $(>10 \mathrm{~cm})$ in 2 . On post-contrast scan the nodes were of homogeneous attenuation in 6 cases and showed necrotic areas in the other 3 . In six cases, the hepatic tumor was in advanced stage and was associated to tumoral thrombus of portal and/or inferior vena cava systems.

\section{SPACE 1514}

Radiologic evaluation in adult constipated and in idiopathic incontinent patients

F. Pomerri (Presenter), S. Curtolo, F. Pittarello, P. C. Muzzio; Padua

Radiology can evaluate not only morphodynamic aspects of the anorectum, but pelvic plane too. Our method needs two complementary times: "phasal" and "dynamic" defecography. In severe constipation 2 groups of patients are identified: patients, middle aged 38.7, without meaningful changes in width of the anorectal angle and in distance between anorectal angle and pubococcigeal line; patients, middle aged 63.3 , show a meaningful reduction in width of the anorectal angle on straining and an increasing of distance of anorectal angle from pubococcigeal line on squeezing. Constipated patients reveal a narrow anal canal $(60 \%)$, rectocele $(42.6 \%)$, mucosal prolapse $(27.8 \%)$, rectal prolapse $(18 \%)$, solitary ulcer $(14.7 \%)$. In idiopathic incontinent patients, middle aged 63.3, we found a lowering of anorectal region on squeezing and, in the most serious ones, also at rest, with the patient sitting. Incontinent patients reveal, in dynamic defecography, a larger anal canal $(67.6 \%)$, rectocele $(36.7 \%)$, mucosal prolapse $(14.7 \%)$, rectal prolapse $(11.7 \%)$. In conclusion, in the elderly constipated patients, we found a deficiency in contractility of pelvic floor's muscles and, in serious incontinent ones, also a deficiency in tonicity of the pelvic floor.

\section{SPACE 1515}

Pancreatic involvement in non-Hodgkin-lymphoma: characteristic feature in CT?

L. Prayer (Presenter), H. Schurawitzki, R. Mallek, F. Karnel, G. Mostbeck; Vienna

Purpose: To describe a possible characteristic CT-feature of pancreatic involvement in patients with Non-Hodgkin-Lymphoma (NHL).

Patients and methods: CT scans of 10 patients with surgically proved manifestations of NHL in the pancreas were compared to examinations performed in patients with carcinoma $(n=25)$ or acute $(n=25)$ and chronic $(n=25)$ inflammatory disease of the pancreas.

Results: In all patients with NHL of the pancreas, the relatively large, unsharply defined, nearly homogeneous hypodense pancreatic masses infiltrated the retroperitoneal fatty tissue and surrounding organs. After i.v. administration of contrast medium, a slight to marked uniform enhancement was noticed. No calcifications were seen.

Conclusions: The described CT-feature is not specific but highly suggestive of pancreatic involvement in NHL. Because of the different management of patients with NHL, neoplastic or inflammatory pancreatic disease, accurate and rapid diagnosis is essential.

\section{SPACE 1516}

CT evaluation in liver transplantation

A. Ramos (Presenter), A. Medina, J.Galobardes, R. Villar, J. Cantarero, F. Lozano, A. Arenas; Madrid

In the evaluation of postoperative complications of hepatic transplantation clinical and laboratory data usually do not provide a specific diagnosis. Thus imaging procedures are pivotal in characterizing parenchymal, biliary or vascular complications.

The CT findings in the abdomen after liver transplantation as seen on 37 examinations in 33 allograft recipients are described. CT was most useful in the evaluation of parenchymal abnormalities of the allograft and in detection of extrahepatic fluid collections. Low density foci within the allograft parenchyma were seen in 18 patients $(54 \%)$ and were associated with infection (4 cases), infarction and necrosis secondary to vascular insufficiency (6 cases), bilomas ( 7 cases) and one pseudoaneurysm that was causing a porto hepatic shunt. Localized extrahepatic fluid collections were present in 17 cases $(51 \%)$ corresponding to 6 bilomas, 1 hematoma and 7 free peritoneal fluid collections. 3 of the focal fluid collections were infected. Since the nature of these fluid collections could not always be determined by CT characteristics, fine needle aspiration was required in 8 cases for a specific diagnosis. Catheter drainage of fluid collections was performed using CT in 6 cases.

$\mathrm{CT}$ is a noninvasive method in the evaluation of postoperative complications that occur after hepatic transplantation.

\section{SPACE 1517}

Smooth muscle tumors of the small intestine: characteristics of their growth and dissemination within the peritoneal cavity

L. Ramos Gonzalez (Presenter), J. Minaya Bernedo, M. Pastrana Ledesma, J. Marcos Lopez, A. García Súarez; Madrid

The behavior of smooth muscle tumors of the intestine (leiomyoma, leiomyoblastoma, leiomyosarcoma) is of special interest because of the characteristics of their growth and dissemination. The aim of this communication is to assess the radiologic findings of their dissemination, both contiguous and 
distant, within the abdominal cavity.

Thirty-five smooth muscle tumors of the intestine have been studied by means of ultrasound (US), computed tomography (CT) scan and magnetic resonance imaging (MRI) to assess the features of the internal structure of the primary tumor and of the intraperitoneal metastases.

They consist of one or more nodules formed by smooth muscle fibers and have a whorled appearance. Their marked tendency to degenerate at the center and the subsequent cystic transformation produce their known radiologic aspect. However, we demonstrate the frequency of the multicystic transformation and the multipartitioned aspect that the primary tumors show on occasion. Nonetheless, the most interesting finding is that these masses grow by producing peripheral nodules which infiltrate neighbouring organs, detach and, by way of the peritoneum, are implanted far from the primary tumor. They also undergo cystic degeneration with one or more cavities, depending on the number of nodules.

These US, CT and MRI findings are virtually pathognomonic signs, and can lead to the exact diagnosis of this type of abdominal tumoral process.

\section{SPACE 1518}

\section{A splenic hamartoma: diagnostic problem}

R. Zissin, V. Rathaus (Presenter); Kfar Saba

We present a case of splenic hamartoma presented with asymptomatic anemia and splenomegaly in a 47 years old woman.

Imaging studies (CT and US) revealed an enlarged spleen with an inhomogeneous mass in it and a focal gross calcification in the hilus. The diagnosis was referred also after fine needle aspiration guided by US and splenectomy was necessary.

We propone that splenic hamartoma should be included in differential diagnosis of a splenic mass.

\section{SPACE 1519}

Pancreatic pseudocyst: interventional radiology

J. L. Raya (Presenter), E. Añorbe, M. D. Martorano, A. Muñoz, F. Lozano, A. Arenas; Madrid

Percutaneous drainage of pancreatic pseudocysts with image control has become an alternative to the open surgery. To the date, pseudocysts in 37 patients have been drained in our service. In this exhibit, we show the technique and indications for pseudocyst drainage. The management is as follows.

Once a pseudocyst is diagnosed, we follow it with U.S. or C.T. for 2 to 4 weeks. If the pseudocyst remains larger than $4 \mathrm{~cm}$ or it is symptomatic, the intervention is instituted. First, we perform a fine needle (22-gauge) aspiration Afterwards, a iodinated contrast or an isotope should be instilated in the cavity to demonstrate communication with the ductal system. An endoscopic retrograde pancreatography can be performed with the same purpose.

1. If a communication is not demonstrated, the simple aspiration is considered adequate.

2. If the pseudocyst relapses after (1), a communication is demonstrated, or in every case when the pseudocyst is infected, a catheter has to be placed. (We use a tandem trocar-catheter insertion). The duration of the drainage ranges from 1 to 4 weeks in our experience. If the pseudocyst relapses, an open surgery is indicated.

\section{SPACE 1520}

\section{Color flow Doppler of visceral organs and non occlusive vascular} disease

Stanton J. Rosenthal (Presenter), Linda Harrison, Louis H. Wetzel, Error Levine, Donald A. Eckard; Kansas City, KS

Purpose: This exhibit illustrates and addresses the uses and limitations of color Doppler imaging in the evaluation of visceral disease and nonocclusive vascular lesions throughout the body.

Methods and Materials: Color Doppler evaluation of parenchymal disease requires special attention to technical details to optimize the visualization of low-flow states.

Results: Major applications discussed include: Renal Pathology - evaluation of the vascularity of renal carcinomas, detection and evaluation of renal vein and IVC tumor thrombi, inflammatory etiologies of renal hypervascularity, renal vascular disorders, evaluation of transplant flow parameters and arterial venous fistulas; Testicular and Penile Pathology - evaluation of testicular masses, differentiation of epididymitis from testicular torsion, evaluation of vasogenic impotence; Obstetrics - differentiation of trophoblastic disease from hydropic degeneration of the placenta, evaluation of placenta percreta, evaluation of vascular fetal masses: Bowel Pathology - evaluation of appendicitis and inflammatory bowel disease: Adenopathy - evaluation of the spectrum of vascularity in reactive adenitis, differentiation of hypervascular adenitis from pseudoaneurysm, differentiation of an accessory spleen from lymphadenopathy; Hepatic Pathology - evaluation of portal hypertension, portal venous thrombosis, differentiation of dilated bile ducts from adjacent vascular structures, evaluation of hepatic masses, vascular encasement by hepatic malignancy, evaluation of portal venous aneurysm; and Aneurysms and Vascular Fistulas - evaluation of mycotic aneurysms, pseudoaneurysms, and arterial venous fistulas.

\section{SPACE 1521}

Small bowel double contrast enteroclysis (SBE) in the evaluation of strictureplasty for Crohn's disease

C. Bosio, E. Juliani, F. Russo (Presenter), R. Zatteri; Torino

Strictureplasty is a recent surgical procedure that allows repair of chronic intestinal strictures due to Crohn's disease, without the need for bowel resection.

This surgical treatment consists, as in pyloroplasty, of a longitudinal incision across the stricture and transverse suturing. Different surgical approaches, according to different pathological situations, are adopted: Heineke-Mikulicz, Finney, Judd and Mosche-Walske-Neumayer techniques.

Twelve patients with fibrotic small bowel strictures secondary to long-standing Crohn's disease underwent a total of 32 strictureplasties from November 1985 to December 1990.

At least one SBE was performed in all cases before and after surgery; radiological follow-up period ranges from 1 month to 4 years after surgery.

Preoperatory SBE showed every single Crohn's stricture, as confirmed at surgery; furthermore early postoperative SBE demonstrated the exact sites of plasties and good results of intervention in all cases.

18 and 25 months after surgery SBE showed recurrence at strictureplasties' sites in 2 patients, who underwent reintervention.

SBE has proven to be the method of choice in evaluating the extent of Crohn's disease before and after surgery.

\section{SPACE 1522}

Intestinal tuberculosis in AIDS patients

E. Salvadó (Presenter), P. Luburich, N. Bargalló, C. Nicolau, C. Cardenal, F. Gimeno; Barcelona

Purpose: To describe the radiologic and computed tomographic (CT) features of intestinal tuberculosis (IT) in AIDS patients.

Methods and Materials: Six HIV seropositive male patients, (age range 24-46 mean 31) were referred for evaluation of fever, diarrhea or abdominal pain. All of them were intravenous drug users. Barium enema (6), barium small-bowel study (4) and CT (3) were performed. Tuberculosis in extrababdominal sites (sputum, cervical adenopathy) was documented by acid-fast smear or culture in all cases, and in two of them biopsy specimens from omentum and terminal ileum showed granuloma formation. Good clinical response to antituberculous chemotherapy was observed in all but one case, which presented intestinal obstruction requiring surgical treatment.

Results: Barium studies showed involvement of terminal ileum (6), cecum (4) and duodenum (1). Thickened folds (4), nodularity (5), ulcerations (2), spasticity (2), ileal structures (1), ileocecal valve gaping (5) and retracted cecum (3) were seen. On CT, one patient had mild ileal wall thickening. Two, had more characteristic findings: severe wall thickening involving terminal ileum, valve and medial side of cecum with regional lymphadenopathy. One patient had massive low-density peripancreatic adenopathy with duodenal involvement and fistulization. Other patient had ascites and irregular soft tissue densities in the omental area (Tuberculous peritonitis).

\section{SPACE 1523}

Generalized emphysema as a complication therapeutic colonoscopy A. Sanchez Marquez (Presenter), M. Gil Garcia, E. Andia Navarro, J. M. Pellicer Punyed, S. Llaverias Borrell, A. Anguera Bosque; Barcelona

Although pneumoperitoneum is a well recognized complication of colonoscopy, to our knowledge, no case of pneumoperitoneum, pneumoretroperitoneum, pneumothorax, pneumomediastinum and subcutaneous emphysema has been described as a complication of therapeutic colonoscopy. A 37-year-old woman with colonic adenomatous polyps, underwent therapeutic colonoscopy, noted chest pain and minimal respiratory difficulty. At physical examination there was no evidence of peritoneal irritation, but chest and abdominal plain film revealed pneumoperitoneum, pneumoretroperitoneum, pneumothorax, pneumomediastinum and subcutaneous emphysema. The patient was managed nonsurgically, showed improvement and the air disappeared after seven days.

It's important to recognize this complication and to differentiate it from true colonic perforation, where surgery will be imminent. 


\section{SPACE 1524}

CT and barium correlation in duodenal tumours and pseudotumours C. Sanchez Torres (Presenter), A. Alvarez Castells, J.Alvarez Fdez, J. Andreu, J. A. Jimenez, S. Pedraza; Barcelona

We attempt to define the utility of adding CT scaning to the barium radiographic evaluation of local mass lesions involving duodenum. Every mass in the duodenal area should be evaluated cautiously. Errors may result in misinterpretation of pancreatic disease, unopacified duodenal diverticulum, vascular structures, and extrahepatic bile duct abnormalities.

Nineteen patients with mass lesions involving duodenum were studied retrospectively.

We present radiological images combined radiographs and CT scans of distinct tumoral and pseudotumoral processes of duodenal area such as duodenal primary adenocarcinoma, lymphoma, colonic metastasis, pancreatic, renal, and gallbladder neoplasms with duodenal invasion for contiguous spread, ampulomas, duodenal divertivula mimicking tumours and a rare case of duodenal neurofibroma in a patient with Von Recklinghausen's disease.

CT and barium studies analyzed separately can be misinterpreted. However, a correct correlation between both and clinical findings allows a diagnostic accuracy and avoid misleading.

\section{SPACE 1525}

Videofluoroscopy of disordered esophageal motor activity W. Schima (Presenter), G. Stacher, K. Uranitsch, P. Pokieser, D. Nekahm, D. Tscholakoff; Vienna

Purpose: Esophageal motor disorders are best evaluated by manometric studies, which, however are time-consuming and not generally available. We investigated if videofluoroscopic studies of esophageal body and lower esophageal sphincter yield results comparable to those obtained manometrically. Methods and Materials: Videofluoroscopy (Sirecord recorder) was performed in a standardized fashion with up to three swallows in the prone oblique and one swallow in the upright position. 29 patients $(7 \mathrm{~m}, 22 n$ with dysphagia $(n=15)$, globus sensation $(n=5)$, noncardiac chest pain $(n=4)$ or scleroderma without symptoms $(n=5)$ were referred to radiography and manometry, carried out 0 to 64 days apart $($ median $=1 \mathrm{~d})$. Data were analysed independently and without knowledge of the results of the respective other examination.

Results: In 2 pts. manometry was not possible. Manometry was normal in 7 and abnormal in 20 pts. (Achalasia, 7 ; nonspecific contraction abnormalities, 7 ; hypo- or amotility in smooth muscle esophagus, 6). Videofluoroscopy had a sensitivity of $80 \%$ (16/20 pts.) and a specificity of $100 \%$ (7/7 pts.). Videofluoroscopy identified 4 of 7 patients with achalasia, 6 of 7 with nonspecific contraction abnormalities and all with hypo- or amotility in scleroderma.

Conclusions: It is concluded that videofluoroscopy is a valuable, although not perfect tool to screen for disordered esophageal motor function.

\section{SPACE 1526}

Radiologic-pathologic correlations of small bowel mucosal surfaces G. Schmutz (Presenter), B. Bui, D. Vetter, M. Y. Jeung, A. Gangi, P. Gagné; Sherbrooke, Québec

Purpose: To determine if the visualisation of small bowel mucosal surfaces during enteroclysis contribute to the pathologic differential diagnosis.

Methods: Over the past 25 months (March 1988-April 1990) we have performed 520 air double contrast enteroclysis with attention being focused on the fine mucosal surface of the small bowel.

Results: In $22 \%$ of our normal small bowel studies, we found a granulanity of the mucosal surfaces. In $63 \%$ of the cases with mucosal lesions of the small bowel (Crohn's disease, NH lymphoma, Celiac disease, Whipple's disease, immunoglobuline deficiency, radiation injury, ischemic enteritis and miscellaneous lesions) we found several mucosal surface patterns: granulanity, recticular pattern, "areolar" pattern and nodular pattern. The pathologic correlation (biopsies and surgical resection) of these radiological features showed modifications of the villous mucosal surface. In the "areolar" pattern there was an inflammatory or tumoral infiltration with partial villous atrophy. In the recticular pattern, we only found inflammatory processes, whereas in the nodular pattern, lymphoid infiltration and/or abnormal mucosal deposits were found.

Conclusions: These modifications of the small bowel mucosal surface pattern should be considered along with the usual radiological features and should be helpful in pathologic differential diagnosis.
SPACE 1527

Ileal complications of sigmoid diverticulitis

P. Gagné, G. Schmutz (Presenter), B. Bui, F. R. Plante, R. K. Chhem, B. Curpen; Sherbrooke, Québec

Purpose: To illustrate and to demonstrate rare complications of sigmoid diverticulitis with different radiologic meanings.

Methods and Materials: Between June 1987 to November 1990, in a series of 82 patients with clinical sigmoid diverticulitis we regrouped 9 patients with small bowel complications. Each patient underwent barium small bowel examination, barium enema, abdominal ultrasound and CT scan in 3 cases.

Results: The findings of small bowel examination were: fistula (4 cases), stenosis ( 3 cases), tumoral appearance ( 1 case) and peritoneal adhesion ( 3 cases). Ultrasound examinations were positive in 6 cases and CT scan in the 3 cases. Barium enema were abnormal in all the 9 patients.

Conclusions: Small bowel manifestations may reveal a sigmoid diverticulitis.

\section{SPACE 1528}

ESWL and transhepatic removal of sludge as adjuvants in local lysis of biliary concrements

K. A. Schumacher (Presenter), P. Janowitz, W. Swobodnik, J. Wechsler; Uim

Purpose: Gallbladder concrements exhibiting high concentrations of cholesterol can be dissolved by methyl tert.-butylether (MTBE) instilled via transhepatically inserted catheters (local lysis, LL). In many cases, however, non-soluble concremental residues cannot be completely removed. Therefore, extracorporal shock wave lithotripsy (ESWL) and post-LL mechanical removal were employed to increase the efficacy of the therapeutic procedure. Methods: The study was performed on 15 patients with multiple non-calcified gallbladder stones. ESWL (Lithotriptor MPL 9000, Dornier) was applicated 3 days prior to the onset of LL. The dissolution procedure employed catheter systems of 5-7 F. Sludge and/or residual concrements were removed under fluoroscopic and/or endoscopic guidance via introducer systems (Amplatz) of up to $14 \mathrm{~F}$. The transhepatic canal was obstructed by fibrin sticker. The intervention was carried out under local analgesia.

Results: Pretreatment of gallstones by ESWL significantly reduced the duration of $L L$ and resulted in smaller concremental remnants, which could be flushed out and/or extracted. Major complications, e.g., biliary leckage of bleeding were not observed.

Conclusions: The combination of LL with ESWL and post-LL mechanical extraction of remnants can be suitable adjuvants in the transcatheter dissolution of biliary concrements.

\section{SPACE 1529}

Quantitative 31P MR spectroscopy of phosphate metabolism in the normal and cirrhotic human liver following fructose administration C. Segebarth (Presenter), T. Metens, R. Longo, A. Grivegnée, J. M. Cucchi, M. Adler, P. R. Luyten, J. A. den Hollander; Bruxelles

Purpose: To assess whether 3 I P MR spectroscopy following a fructose load has potential as a clinical test of hepatic function.

Introduction. The administration of fructose induces changes in the concentratires of the phosphorous metabolites in the human liver, which can be monitored by means of 3IP MR spectroscopy. This has raised the expectation that 3IP MR spectroscopy following a fructose load might eventually constitute the basis of a test of hepatic function. In this study, we have assessed whether a number of prerequisites to the clinical use of the fructose load $31 \mathrm{P}$ MR examination are met.

Methods: $1 \mathrm{H}$ image-guided 31P MR spectroscopy was applied. Reproducibility was assessed by repeating the fructose load 31P MR examination 5 times on one particular healthy individual, over a period of several months. Biological variability of the 3 IP MR response was evaluated on a population of 10 healthy individuals. Clinical applicability was verified by examining 10 patients suffering from cirrhosis. The $31 \mathrm{P} \mathrm{MR}$ spectra were measured sequentially, every 2 minutes. The spectral parameters were determined by fitting the 3IP MR signals by means of a model function.

Results: The changes in the tissue $\mathrm{pH}$ and in the hepatic concentrations of fructose-1-phosphate, adenosine-triphosphate and inorganic phosphates could be monitored very accurately and reproducibly in the normal liver. The measurements performed on healthy individuals exhibit a characteristic normal response. Cirrhosis patients exhibit a response which is significantly different on the average from that of the healthy volunteers. The cirrhotic livers generate reduced phosphate signal intensity, rendering difficult the interpretation of individual patient examinations.

Conclusions: The accuracy and the excellent reproducibility of the 31P MR response observed with the normal liver suggest that 3IP MR spectroscopy following a fructose load has potential as a clinical test of liver function with hepatic disorders which do not cause a marked reduction in phosphate signal intensity. 


\section{SPACE 1530}

Chole-CT, new contribution for the study of choledocho X. Serres (Presenter), I. Pont, R. Bassaganyas, J. C. Vilanova, J. J. Teruel, M.D. Figueras; Girona

A new technique is presented in order to improve the visualization of the choledocho by C.T. which is based on the knowledge of oral cholecystography. The study has been carried out on a group of 20 patients without a known hepatic pathology or digestive pathology. An oral cholecystographic contrast was administrated to all these patients. We carried out tomographic sections before and after giving them a preparation rich in proteins in order to visualize the contrasted choledocho. In some cases we have done oblique reconstructions above the choledocal section. Of the 20 patients without a known hepatic pathology or digestive pathology we got the opacification of the bladder from 14 of them and we got the opacification of the choledocho from 10 of them. Of the 10 patients with pancreatic pathology we got the opacification of the bladder from five of them and the opacification of the choledocho from four of them. With this non aggressive, economic and without side effects technique we can see the choledochal section by C.T. although the choledocho is not dilated. This technique is also applicable to mild jaundice and in those cases were there is pancreatic pathology.

\section{SPACE 1531}

\section{CT-portography in the postoperative management of patients with} colo-rectal tumors

A. J. Smeets (Presenter), H. Pieterman, A.I. J. Klooswijk; Rotterdam

Purpose: To evaluate diagnostic methods for the accurate assessment of the presence and localization of metastases of the liver in patients with colo-rectal tumors, assuming that complete recovery may result after metastasectomy in these patients.

Methods: In our hospital the post-operative follow-up of these patients is: periodical sonography of the liver, - and determination of CEA blood levels. If one or both of these tests are positive, CT-portography is performed to establish the presence of metastases in the liver and subsequently be informed about the precise number of metastases and their localization related to the vascular and bile tree.

Results: Of 15 patients are presented, 5 patients (33\%) appeared to be inoperable on the basis of CT-portography. In 4 patients CT-portography demonstrated more metastases than was shown on US. CT-portography provided better insight in the anatomical situation of a solitary metastasis in 1 patient.

Conclusions: CT-portography, however invasive, is a valuable examination in the postoperative management of patients with colo-rectal tumors, and may prevent unnecessary operations.

\section{SPACE 1532}

Fibrolamellar hepatocellular carcinoma: evaluation with multimodality imaging

Ph. Soyer (Presenter), A. Roche, M. Levesque, P. Legmann; Colombes

Purpose: To describe the ultrasonographic (US), arteriographic, CT and MR characteristics of the fibrolamellar (FL) subtype of hepatocellular carcinoma (HCC).

Methods and Materials: Ten patients (mean age, 28 years) with pathologically proved FL HCC were retrospectively studied. The patients were investigated by US $(n=10)$, coeliomesenteric angiography $(n=9), C T(n=9)$ and MR $(\mathrm{n}=3)$.

Results: The tumors were solitary $(7 / 10)$, well delineated $(7 / 10)$ and hypervascular $(8 / 10)$. A central scar $(n=3)$, calcifications $(n=3)$, dilated intra hepatic ducts $(n=2)$ and thrombosis of a segmental portal branch $(n=1)$ were demonstrated using the multimodality imaging techniques. Lymph node involvement $(n=3)$ was only shown by CT. In our series, CT was the most accurate technique for staging and diagnostic approach.

Conclusions: This report emphasizes the wide variability of imaging features of FL HHC and the predominant role of CT for suggesting the diagnosis.

\section{SPACE 1533}

Dysphagia in stroke patients - a prognostic factor?

Anne Sparenberg (Presenter), Andreas Bock, Maria Jockers, Peter Vogel, K.-J. Wolf; Berlin

Purpose: The relationship between dysphagia in its various forms and the topography of infarcted areas has not yet been investigated. Another question is how far dysphagia following apoplexy should be considered a prognostic factor for the complication rate.

Methods and Materials: In a prospective study, 25 stroke patients were examined after barium swallow using a digital cinefluorography unit (25-50 images/sec; Polytron, Siemens). All stroke lesions were confirmed by MRI or
CT.

Results: $1.25 \%$ of these patients suffered from dysphagia. 2 . The incidence of dysphagia was not related to left vs. right hemisphere strokes. 3. Aspiration occurred in 5 of 25 patients. 4. Dysphagia patients were hospitalized considerably longer than others.

Conclusions: Dysphagia patients have a markedly higher complication rate (aspiration, pneumonia), and dysphagia should thus be considered a prognostic factor in stroke patients.

\section{SPACE 1534}

Ultrasonographic findings in four cases of xanthogranulomatous cholecystitis

M. Sperandeo (Presenter), A. Varriale, G. Sperandeo, M. Faruolo; Napoli

Xanthogranulomatous cholecystitis (XGC) is a rare form of cholecystitis characterized by yellow nodules in a thickened gallbladder wall. It is often associated with gallstones, which are believed to be significant aetiological factors.

We report four cases of XGC, in which ultrasound examinations permitted a pre-operatively diagnosis. In all patients the main presenting symptom was a right upper quadrant abdominal pain accompanied by fever, nausea and vomiting in three patients. Leukocytosis, hyperbilirubinemia and elevated alkaline phosphatase were found in two patients. Both oral cholecystogram and intravenous cholangiogram failed to image the gallbladder successfully. Ultrasonography revealed a distended gallbladder with thickened walls and irregular mucosal surface: within the gallbladder lumen a non-homogeneous content and gallstones were detected. These findings were similar in all the cases, suggesting an important role in the differential diagnosis with the cancer of the gallbladder. Infact the thickened wall was always well defined respect to the surrounding liver tissue; the mucosal surface was irregular in all the gallbladder and not limited to a little area: the gallbladder lumen was well detectable; all these findings are hardly revealed in the case of a cancer of gallbladder. In conclusion, XGC must be taken in consideration in the case of symptoms suggestive of a cancer of the gallbladder; ultrasonography may be an effective tool in differentiating the two diseases before surgical intervention, significantly affecting the prognosis.

\section{SPACE 1535}

Referrals to double contrast colon examination: a general practitioner's view Siri Steine (Presenter); Oslo

It is generally recognized that the double contrast colon examination of the large bowel (DCE) is very useful in the detection of colorectal cancer and polyps.

The majority of the performed DCE in Norway is initiated by general practioners.

The aim of this study is to relate the occurrence of specific symptoms, anamnestic/biological data, and symptom duration, to the radiologic findings of neoplasms.

2414 consecutive patients, examined with DCE were evaluated radiographically. 1852 patients answered a questionnaire indicating symptoms, their duration and other anamnestic information.

The prevalence of polyps was $11 \%$ and of cancersuspect tumors $3 \%$ in this material.

In bivariate analysis the following was found:

1. Significant association between polyps and age, family history of cancer, earlier polyps, and rectal bleeding.

2. Significant association between colorectal cancer and age, sex, earlier colorectal cancer, rectal bleeding and weight loss.

The further plan is to study the association in a multivariate setting, and to investigate which groups of factors are important for predicting the existence of colorectal neoplasms. This may become a tool for doctors when selecting patients for further investigation.

The results will be available in time for the congress.

\section{SPACE 1536}

\section{Ultrasound of the splenic cysts}

T. Siniluoto, M. Päivänsalo, S. Lähde, P. Lohela, I. Suramo (Presenter); Oulu

Nonparasitic splenic cysts are rare (about $0.05 \%$ in an autopsy material). There is only a little knowledge about the US findings and natural course of the cysts. With a material of 50 patients ( 46 solitary and 4 multiple cysts) we have systematically examined the US findings. 29 patients had also follow-up US examinations (mean interval 32 months).

The size of the cysts ranged form $0.8 \mathrm{~cm}$ to $15 \mathrm{~cm}$ (mean $3.7 \mathrm{~cm}$ ). $62 \%$ were anechoic, $12 \%$ hypoechoic, $8 \%$ isoechoic and $2 \%$ showed mixed echogenicity. In $4 \%$ the content was invisible because of heavily calcified walls. The majority of large cysts $(>5 \mathrm{~cm})$ were echogenic and small cysts anechoic. $20 \%$ had calcified walls. The mean diameter of calcified cysts was $6.2 \mathrm{~cm}$ and noncal- 
cified $3.0 \mathrm{~cm}$.

At follow-up the size of the cysts remained unchanged in $62 \%$, market increase in the diameter was seen in $7 \%$ and a slight increase in $21 \%(10 \%$ had been aspirated. There was a spontaneous appearance of a second cyst in $7 \%$ and a $1.8 \mathrm{~cm}$ and $\mathrm{a} 1.5 \mathrm{~cm}$ cyst disappeared.

In spite true cysts were very rare in the material, a history of trauma was available in only 2 patients.

\section{SPACE 1537}

Percutaneous drainage of pancreatic pseudocysts as an alternative of the surgical treatment

J.Szmeja, K.Rzymski, A.Lemke, K. Linke, I.Szpakowska-Rzymska (Presenter), J. Rosiński; Poznań

Eighty-five patients with pancreatic pseudocyst were treated by percutaneous drainage in our Department between 1984-1990. All patients had Endoscopic Retrograde Cholangiopancreatography (ERCP) and abdominal ultrasonography to determine the size and place of the pseudocyst. Cyst biopsies with percutaneous drainage were taken in 80 cases (we use Cystofix Braun) and percutaneous cystogastrostomy were done in 5 cases (GUPC-8, 5-10-PE). Single drainage procedure was the sole treatment in 65 cases, the other 20 patients needed two or maximum three times drainage.

We conclude that percutaneous drainage is safe and simple offers good treatment of pancreatic pseudocyst.

\section{SPACE 1538}

Value of ultrasonography in the management of pericholecystic abscess Vassil Zefov, Atanass Talaganov (Presenter); Plovdiv

Presented are ultrasonographic findings in four cases with a pericholecystic abscess. Two cases are classified as having pericholecystic abscess in the gallbladder bed and the two other have abscess with intramural location. Demonstrated are some difficulties in the ultrasonic differential diagnosis within these two types. All four cases underwent surgical exploration. Finally comparision between classical X-Ray diagnosis (plain films, cholecystography), ultrasound and surgical findings regarding the management of the pericholecystic abscess is performed.

\section{SPACE 1539}

The CT-Sellink, a new method to determine the diagnosis M. Crohn Jens Thiele (Presenter), R. Klöppel, H.-G. Schulz; Leipzig

Enteroclysma after SELLINK in a modified form is a proven method in the small intestine imaging. With the help of thin barium suspension (Fa Nicolas, Micropaque (T) and a distensing medium it allows us to have an excellent vision of the small intestine.

On the basis of 50 examinations we report on the method and its capacity in the diagnostic of inflammatory small bowel diseases especially concerning Crohn's disease in relation to clinical and histological analysis.

\section{SPACE 1540}

Newly devised contrast media for double contrast barium enema, low density barium sulfate suspension with high adhesion

Yoshito Tonariya (Presenter), Hiroaki Shimizu, Yukito Wada; Saitama

Purpose: To evaluate double contrast barium enema using newly devised low density contrast media with high adhesion.

Methods and Materials: $400 \mathrm{ml}$ of the contrast media comprising an aqueous suspension containing $30 \mathrm{w} / \mathrm{v} \%$ of barium sulfate and 2.0 to $0.5 \mathrm{w} / \mathrm{v} \%$ of gum tragacanth was used for double contrast barium enema of 25 men and 25 women aged 26-80.

Results: Even in the overlapping portion of intestinal tubes or pooling portion of barium sulfate, transmissivity optimal for observation of mucosal and peripheral image can be obtained, whereby minute lesions will never be overlooked.

Adhesion of contrast media can be effected simultaneously with passing through intestinal tube, and no postural change to great extent is required.

By use of an amount of $400 \mathrm{ml}$, movement of contrast media to the proximal portion of colon is rendered possible. The inspection time is remarkably short. Due to little postural change, effective double contrast barium enema can be done for physically handicapped patients or aged people.

Due to good water retentivity, the contrast media adhered will not be cracked for a long time.

Conclusions: Our method using this contrast media was considered as simpler and better double contrast barium enema, especially for mass screening.

\section{SPACE 1541}

\section{Imaging of ascariasis}

Carlos Eduardo Triana Rodriguez (Presenter), Carlos Jimenez C., Antonio Botero, Patricia Castro; Bogota

Ascariasis is a helminthiasis that infests man by the ingestion of contaminated water or food mainly in undeveloped countries. It is pathogenic for men in the gastrointestinal tract with severe hepatic complications.

We present the different forms of infestation as well as complications - through imagenology.

- Upper GI Series

- Intestinal transit

- Barium enema

- Transparietohepatic

- Cholangiography

- Ultrasonography

\section{SPACE 1542}

Videofluoroscoplc examinations of normal and pathologic deglutition K. Uranitsch (Presenter), P. Pokieser, W. Schima; Vienna

This video presentation (10 to 15 minutes) demonstrates the dynamic appearance of normal and pathologic deglutition examined with videofluoroscopy. Designed as a teaching tool it will cover anatomy, physiology and basic pathology of deglutition. Characteristic appearance of sphincter dyskinesia, early stage of Zenker's diverticulum and intradeglutitive aspiration are presented.

Materials: We investigated patients with symptoms of dysphagia, globus sensation or aspiration with videofluoroscopy using a standardized examination protocol with nonionic iodine contrast medium, liquid barium and barium gelatine balls $(13 \mathrm{~mm})$. The oral and pharyngeal phases were examined in a.p. and lateral projections upright, the esophagus in upright RAO and prone oblique positions.

Videofluoroscopy is documented using a Sirecord (Siemens) and a U-matic (Sony) video tape recorder.

Results: During the oral phase the patient should be able to hold the bolus. Initiating the swallow the tongue propells the bolus backwards while the soft palate closes the nasopharynx. Hyoid and larynx move anterior and superior, giving way to the bolus while the totally inverting epiglottis as well as the true and false vocal chords protect the airways. The pharyngoesophageal segment should be relaxed as the bolus arrives and closes again after the entire bolus has passed without cutting the bolus tail.

The esophagus should be generally cleared with a single peristaltic wave.

\section{SPACE 1543}

Primary hepatic abscess - percutan US diagnostics and therapy Gyula Varga (Presenter), Ferenc Ifi, Zoltán Cseh; Zalaegerszeg

Among 97 abdominal abscesses only some of them were evident in the postoperative period, most were unexpected - without characteristic anamnesis, clinical and laboratory symptoms. At 78 patients abscesses were verified by ultrasonic intervention, 64 of them had been cured by percutaneous punction, 52 resulted complete abscessfree recovery.

7 of 8 "primary" hepatic abscesses of unknown origin had neither characteristic symptoms, nor typical ultrasonic appearance, and the patients were in relatively good condition, observed at ambulatory or medical departments. The early diagnostic needle punction differentiated from malignant tumor, haemangioma or other cystic-solid lesion, resulted in purulent content for laboratory identification. The liquid content of the abscess had been evacuated and the procedures were repeated twice or several times. Single or repeated suction proved satisfactory in 5 cases, while catheter drainage was necessary for the other 3 cases.

One of the above patient needed further surgical intervention, and after a few months two more needed different types of operation in much better condition. The early direct diagnostics and therapeutic procedures resulted recovery for all those 8 patients.

\section{SPACE 1544}

Treatment of small hepatocellular carcinoma by percutaneous Ethanol injection, evaluation after long-term follow-up

R. Vilana (Presenter), C. Bru, J.Bruix, C. Ayuso, X. Calvet, L. Bianchi, R. Gilabert; Barcelona

The engagement of early detection plans based on U.S. and/or A.F.P. determination for H.C.C. has induced a progressive increase in the number of small tumors suitable for surgical treatment.

However, surgical resection or liver transplantation can still be performed only in a reduced proportion of cases. This has prompted the development of other therapeutic modalities such as P.E.I. 
The present study was aimed to assess to evaluate the efficacy and tolerance of P.E.I. in a series of 30 patients with H.C.C. smaller than $4 \mathrm{~cm}$, who have been followed at least 6 months after being treated.

Five of the 30 patients did no complete the treatment. The remaining 25 patients received between 6 and 18 sessions of ethanol injection. The follow-up after treatment range between 6 and 36 months. 13 patients have developed new intrahepatic tumoral nodules. The other 12 cases are still free of disease being regularly checked by US and CT. Thus a complete response to the treatment has been obtained in $48 \%$ of the cases.

Repeated P.E.I. was well tolerated and no major complications were observed.

\section{SPACE 1545}

A study of the contrast enhanced CT of the pancreas - the most avallable procedure for using high-speed $\mathrm{CT}$

Jun Watari (Presenter), Nobuhito Katayama, Yasuo Amano, Taro Ichikawa, Tatsuo Kumazaki, Toshiaki Yokokura, Kinichi Ebata; Tokyo

Purpose: To determine the best procedure of the incremental rapid sequential contrast enhanced CT of the pancreas, a comparative study of various contrast patterns using high-speed $\mathrm{CT}$ was carried out.

Methods and Materials: The incremental rapid sequential contrast enhanced CT of the pancreas was carried out in 70 patients with no pathological pancreatic lesion and time density measurement was done in each patient, using high-speed CT (TCT-900S). The density values following various injection rates $(1 \mathrm{ml} / \mathrm{sec} 4 \mathrm{ml} / \mathrm{sec}$ ) of contrast medium (iohexol) from $300 \mathrm{mgl} / \mathrm{ml}$ to $350 \mathrm{mgl} / \mathrm{ml}$ and injection volume of $100 \mathrm{ml}$ to $130 \mathrm{ml}$ were compared.

Results: The density value of the pancreas was improved significantly with an increase of injection rates and of iodine contents, while no significant difference was noted in the density following the injection of the various volume of the medium. The present study suggested that rapid injection of $100 \mathrm{ml}$ contrast medium with $350 \mathrm{mgl} / \mathrm{ml}$ was the most available procedure for incremental rapid sequential contrast enhanced $\mathrm{CT}$ of the pancreas.

\section{SPACE 1546}

Liplodol-Cisplatin embolisation in hepatocellular carcinoma - radiological technique and evaluation

P. Peene, G. Wilms (Presenter), A. L. Baert, P. Matthys, H. Rigauts, W. van Steenbergen, J. van Isveldt, Y. Breysem, J. Fevery; Leuven

In a one year period, 14 patients with inoperable and bioptically proven hepatocellular carcinoma (HCC) underwent 18 hyperselective catheterisations of the arteria hepatica propria with combined injection of Cisplatin and Lipiodol.

For the 18 catheterisations of the hepatic artery, distal hyperselective catheterisation of the proper hepatic artery in order to avoid Lipiodol embolism to the gastroduodenal, gastric or other anastomic arteries could be achieved using conventional diagnostic catheters ( 5 cases), or, if this was not possible, using an easy to use and non-expensive coaxial catheterisation system (13 cases).

Radiological follow-up of the lesions was performed by means of a C.T.-scan, obtained 24 hours and 6 weeks after chemoembolisation. Further CT follow-up studies were performed depending on the clinical evolution. We report the computed tomographical evolution of 12 patients, which had a CT follow-up of at least 6 weeks. It appears that hypervascular hepatocellular carcinomas have a tendency to diminish in volume, especially those which retain Lipiodol after 6 weeks, and that the survival under IA chemotherapy is lesser in patients with hypovascular tumors that do not retain Lipiodol.

\section{SPACE 1547}

Application of metallic stents in benign strictures of bile ducts Eugenio Zanon (Presenter), Giovanni Gandini, Dorico Righi, Paolo Fonio; Torino

In the last 7 years we have treated 108 patients with benign strictures of bile ducts with percutaneous transhepatic bilioplasty (PTB), by means of Gruntzig balloon catheters.

We obtained an overall success rate of $70 \%$, after at least 18 months of follow-up: these results are similar to the surgical ones.

In the last 15 months, in selected cases, we used auto-expandable (4 cases) or balloon expandable ( 9 cases) metallic stents after PTB.

We obtained good results in 12 of 13 patients treated with metallic stents. Episodes of cholangitis and an increase of cholestasis indexes occurred in 1 patient with sclerosing cholangitis, 3 months after treatment.

The mean period of follow-up was 5 months.

We believe that the use of metallic stents, in selected cases, should ulteriorly improve the results of biliary stricture dilatations.

\section{SPACE 1548}

Color Doppler in the detection of portosystemic collaterals in portal hypertension

P.S. Zouboulis (Presenter), C. Hatzimichail, N. Fronas, N. Gourtsoyannis; Kifissia

Purpose: To evaluate the sensitivity of color and PW doppler for the diagnosis of portal hypertension by demonstration of a spontaneous patent portosystemic anastomosis.

Methods and Materials: We examined 92 patients with histologically proved cirrhosis and portal hypertension with ultrasonically detected spontaneous portosystemic collateral. We compared the ability of color and PW doppler to prove the existence of a patent portosystemic shunt with the ability of conventional sonography alone to detect the anastomosis. 46 of these patients had endoscopically proved esophageal varices.

Conclusions:

$\mathrm{N}^{\circ}$ of patients

\begin{tabular}{lccc}
\hline Location of anastomosis & US & US + PW & US + color + PW \\
\hline gastroesophageal & 17 & 24 & 31 \\
paraumbilical vein & 11 & 14 & 19 \\
splenorenal & 10 & 12 & 17 \\
gallbladder wall & 2 & 4 & 7 \\
intrahepatic & 2 & 2 & 4 \\
spleno-retroperitoneal & 0 & 3 & 5 \\
omphalo-ilio-caval & 3 & 5 & 7 \\
sup. mes. vein IVC & 0 & 0 & $\frac{2}{92}$ \\
TOTALS & $\frac{0}{64}$ & &
\end{tabular}

The addition of color and PW doppler to the conventional US examination significantly increases the sensitivity for the diagnosis of portal hypertension by demonstration of a spontaneous patent portosystemic anastomosis. Color doppler adds information concerning patency and direction of flow mainly concerning unusual and difficult to detect portosystemic shunts.

\section{SE}

\section{SPACE 1549}

Renovascular hypertension: correlation of duplex color Doppler sonography with DVA and IVU in morphofunctional evaluation S. Baldelli (Presenter), G. Argalia, P. Perotti, D. Migliorini, G. M. Giuseppetti; Ancona

Purpose: The purpose of this study is to define the diagnostic value of Duplex Color Doppler Sonography (DCDS) and Echotomography (ECT) in renovascular hypertension diagnosis, in comparison with Digital Venous Arteriography (DVA) and Urography (IVU).

Methods: 84 patients $(54 \mathrm{M}, 30 \mathrm{~F})$ with clinical-laboratory suspicion of renovascular hypertension underwent DVA, to renal arteries evaluation (normal; stenosis $50 \%$; stenosis $50 \%$; occlusion), combined with IVU, to definition of renal function and uro-secretory system integrity. Just before radiographic investigations all patients underwent besides DCDS (Ansaldo AU 590:3.5 MHz) and ECT; for each renal artery detected were evaluated max velocity and resistive index, and for each kidney were evaluated size, parenchymal thickness and echostructure. DVA, IVU and US findings were combined each other and with clinical-laboratory data.

Results: The results show that DCDS demonstrated high sensitivity in flow evaluation when an optimal doppler signal is picked up from renal arteries. On the other hand, when low or trouble signal was detected, no functional evaluation appeared possible. In cases with renal stenosis no good correlations were found between DVA, IVU and DCDS findings. Our experience shows that DCDS is indicated in the first diagnostic approach of disease, but the limit due to impossibility to pick up in all patients an optimal signal suggests its complementary role with DVA and IVU association.

\section{SPACE 1550}

Comparative anatomy of the urinary bladder: MR imaging of normal volunteers and postmortem and postsurgery specimens

Jelle O. Barentsz (Presenter), John M. G. Kauer, Sjef H.J. Ruijs, Gerd Rosenbusch, Theo W. J. Witkamp; Nijmegen

Purpose: To present an atlas of comparative anatomy as shown on MR images of normal volunteers, postmortem specimens, patients with bladder tumors, and surgical biopsy specimens.

Methods and results: The first part described normal MR anatomy of the male 
and female pelvis. In the second part, MR images and corresponding cross sections of anatomic specimens are correlated. Finally, in 11 patients, in vivo and in vitro MR images are compared with macroscopic and histopathologic data on resected specimens.

Conclusions: The MR images provide an excellent insight into the anatomy of the bladder wall and surrounding structures.

\section{SPACE 1551}

Role of color Doppler in the evaluation of renovascular hypertension Libero Barozzi (Presenter), Pietro Pavlica, Francesco Losinno, Alfio Amato, Arcangelo de Fabritiis; Bologna

Purpose: To evaluate the efficacy of color Doppler US in diagnosing renal artery stenosis using angiography as the gold standard.

Methods and Materials: Forty-four patients ( 87 arteries, because 1 patient had been submitted to nephrectomy) referred for renovascular hypertension were scanned prior to angiography with color Doppler. The waveform spectral analysis, peak systolic frequency, resistive index and stenosis index were calculated from waveforms obtained at the proximal renal arteries and at the renal hilum distal to the stenosis. The average time of each examination was 25 minutes.

Results: There were 51 normal arteries and 36 stenosis detected with arteriography. Of the 87 vessels the ultrasonic study was technically inadequate in only 2 vessels $(2.3 \%)$. In the 85 remaining cases we identified $29 / 36$ stenosis and $49 / 49$ normal arteries. Using color Doppler the sensitivity was $80 \%$, specificity $100 \%$ and accuracy $89 \%$.

Conclusions: The study demonstrates that color Doppler imaging may be used in the diagnosis of renovascular hypertension but there are necessary further studies must define the diagnostic parameters, the reproducibility and the intra and inter observer repeatability.

\section{SPACE 1552}

\section{Percutaneous nephrostomy in pyonephrosis}

F. Camuñez (Presenter), A. Echenagusta, G. Simo, J.de la Torre, F. Herranz, C. Hernandez, A. Abellas; Madrid

A series of 120 pyonephrotic kidneys in 117 patients were drained by percutaneous nephrostomy (PN).

All the PNs carried out by the Department of Radiology at our hospital between May 1982, and October 1990, were rewieved. During this period a total of 485 PNs were performed on 465 patients. PN was indicated in 117 patients $(23 \%)$ with pyonephrosis.

In 114 patients, clinical symptoms disappeared 24-48 after procedure. Two patients died from sepsis subsequent to anuria and underlying malignancy. Once the acute phase had remitted, interventional procedures were carried out in 49 cases, and consituted the definitive therapy in 46 . In 62 cases elective surgery was the definitive therapy. In 7 cases it was ESWL. Major complications included 7 cases of sepsis resolved with proper medical therapy.

\section{SPACE 1553}

Signet-Ring cells adenocarcinoma of the bladder, all the different forms depending on their origin and localization

N. Alegre, B. Cortina (Presenter), N. Marcos; Madrid

Purpose: The signet-ring cells adenocarcinoma of bladder is an uncommon entity. They are only 39 cases described in the literature and twenty of them are primary tumors. We show the three possible forms of presentation of the signet-cell adenocarcinoma.

Methods and Materials: We present three cases (primary, metastasic and uracal cyst signet-ring cells adenoma) that illustrate the different origin and clinics manifestations of this uncommon neoplasia.

Results: This kind of tumor is characterized by a submucosal and muscular vesical infiltration with an intense desmoplastic reaction. The cistoscopy can be normal, so the imaging methods are specially important to suspect the process.

The most constant radiologic finding is the thickening of the wall bladder which is identified by U.S. or C.T. The confirmation is made by deep biopsy. The fundamental diagnostic criteria of the uracal adenocarcinoma are the localization on the vesical dome, the extravesical growth and the absence of carcinoma in the remainder bladder.

\section{SPACE 1554}

Percutaneous renal blopsy with U.S. control

B. Cortina (Presenter), N. Alegre, M. Martinez; Madrid

Purpose: We present our experience on U.S. guided renal biopsies on 42 patients. We consider the echography as the best technique to locate the kidney and to choose the place of punction, the two basic elements for a safe renal biopsy.
Methods and Materials: Prior to the punction we explore both kidneys and we choose the best place for puncture. In the vast majority of the cases this was the inferior pole of right kidney. We use a modified Menghini Surecut 15 and a set of punction adapted to a $3.5 \mathrm{MHz}$ transducer.

Results: The mean age was 34.8 years, the mean number of punctions per patient 3.3 and the mean number of cylinders and glomeruli was 2.1 and 16.1, respectively.

The immediate and 24 hours control showed no important complications. The punction was diagnostic in $100 \%$ of cases.

We believe that the diagnostic yield of this technique is superior to blind or fluoroscopy guided renal biopsy. There is a less risk for patients and the technique allows an early control and management of any possible complication.

\section{SPACE 1555}

Diagnostic value of MRI in diseases of the testis and scrotum B. M. Cramer (Presenter), E. Schlegel, J. Thüroff; Wuppertal

Purpose: To determine the value of MRI in diseases of testis and scrotum. Methods: 300 patients with diseases of the testis and scrotum underwent examination on a Siemens Magnetom 1.5 using surface coils. Surgical and bioptic comparison was possible in more than 100 patients.

Results: Malignant tumors of the testis were diagnosed without exception. There were no false-positive or false-negative readings. Embryonal cancers and seminomas showed different morphologies. With some limitations differentiation of Beta-HCG-positive seminomas appears possible. In benign diseases differentiation of inflammatory and other lesions as delayed torsions seems possible. With testicular trauma rupture of the tunica albuginea can be visualized.

Conclusions: MRI is an important tool for diagnosis of diseases of the scrotum and testis, whenever indication for surgery was not clear, MRI greatly helped in decision-making. Also, mm-size tumors were detected and differentiated from other type lesions.

\section{SPACE 1556}

\section{CT imaging in pelvic inflammatory disease}

Roberto Dore (Presenter), Alfredo La Fianza, Lorenzo Preda, Regina Bellantuono, Saverio Tateo, Luciana Babilonti; Pavia

Purpose: Pelvic Inflammatory Disease (PID) is one of the most serious complication of the sexually transmitted diseases. Sonography contributes significantly to the diagnosis and treatment of PID. To assess the role of CT in evaluation of PID, a retrospective analysis of CT findings in 15 women with PID was correlated with pathological findings at surgery or laparoscopy.

Methods: CT features were correlated with the different anatomo-pathologic stages of disease: I - initial salpingitis with edema and hyperemia; II exsudative phase; III - tubo-ovarian abscess; IV - pelvic peritonitis.

Results: In I phase CT demonstrated none abnormalities in adnexa; in some cases there were findings suspecting pyometra. In II phase adnexa appared wide and enlargement of salpinx was visualized. In III phase enlarged salpinx were never separated from adnexal mass; there was contrast enhancement ring: mass and uterine borders vanished and surrounding soft tissue became inhomogeneous. In IV phase fascial and peritoneal thickening, inhomogeneous and increased density of pelvic adipous tissue, extragenital structures involvement and inhomogeneous and poor-defined masses were described. Conclusions: CT features of PID had good specificity in advanced stages especially when disease appeared as pseudoneoplastic clinical masses and in order to evaluate extragynaecological involvement in presurgical evaluation.

\section{SPACE 1557}

Percutaneous management of ureteral fistulas

A. Echenagusta (Presenter), F. Camuñez, G.Simo, J.de la Torre, A. Abellas, F. Herranz, J. M. Diez Cordero; Madrid

Purpose: We reviewed our experience with 22 patients who had ureteral leaks to assess the role of percutaneous urine diverting procedures in their treatment. Methods and Materials: The location of the leak was ureteropelvic junction in 5 patients, proximal ureter in 3 , distal ureter in 11 , and ureteroileal anastomosis in 3 . In 20 patients the fistulas resulted from surgical injury. In all patients the fistulas were treated by percutaneous nephrostomy or ureteral stenting.

Results: One patient died 2 weeks after treatment. Another patient was operated 3 days after treatment. In 10 of the remainder 20 patients, the ureteral fistula healed without development of a stricture or further intervention during a follow-up from 6 months to 2 years. The results were more favorable in patients with underlying nonneoplastic disease (success in 8 of 13 cases) than in patients with underlying neoplastic disease (success in 2 of 7 cases). The ureteropelvic junction was the most favorable location of the leaks (success in 4 of 5 cases). In the patients treated by ureteral stenting the success rate was $77 \%$, while in the patients treated by percutaneous nephrostomy this rate was $27 \%$. 


\section{SPACE 1558}

MRI and transurethral ultrasound in urinary bladder neoplasms staging P. Ercolani (Presenter), E. De Nigris, M. Misericordia, A. Villanova, A. Giovagnoni; Ancona

Purpose: The purpose of this study is to evaluate the diagnostic accuracy of MRI and transurethral ultrasound in urinary bladder neoplasms staging. Methods: 22 patients ( $20 \mathrm{M}, 2 \mathrm{~F}$ ), age ranged from 46 to 73 years with bladder neoplasms proved by conventional methods (I.V.U., US, TC, Endoscopy) underwent transurethral US (Kombison 320-5), and MRI examination using a superconductive 1.0 T scanner (Magnetom). Multiplanar SE T1 and T2 weighted images of the pelvis were obtained. MRI and US findings were correlated each others and with the histopathological ones after surgical treatment (6 cystectomy, 8 partial resection, 8 T.U.R.).

Results: US correctly staged 16 cases, overstaged 2 cases (T3a): understaged 4 cases (T2, T3b). MRI correctly staged 18 cases, overstaged 2 cases (T2, T3a) and understaged 2 cases (T3b). Association of two methods provided an overall accuracy of $91.6 \%$.

Conclusions: Our experience confirms transurethral US value in detecting small lesions. On the other hand MRI permits a better evaluation of perivesical fat and pelvic organs infiltration.

\section{SPACE 1559}

\section{Balloon catheter dilatation of male urethral strictures}

A. Garcia Suarez (Presenter), J. L. Perez Picouto, C. Gonzalez Hernando, P. Fraga Rivas, J.Minaya Bernedo, M. lllanas Sanz, A. Matallana Hernandez; Madrid

Urethral strictures constitute a significant clinical problem that often requires long-term management. Bouginage, urethrotomy, and urethroplasty are the current therapeutic cornerstones.

We report our experience using balloon catheter dilatation of urethral strictures in 33 patients, the patients were 17-72 years old. All the strictures were diagnosed by cystourethrography antegrade and retrograde. The etiology of strictures were 4 traumatic stricture of the membranous urethra, 6 caused by gonorrhea ( 3 of the penile urethra), 15 iatrogenic stricture of the bulbous and membranous urethra due to prolonged catheterization and 8 post-urethroplasty or post-urethrotomy. Catheters 5 French with balloons that were 4-12 $\mathrm{mm}$ was inflated, for 3-5 min with intervals of $1-2 \mathrm{~min}$ between inflations. The patients were followed for 4-24 months. The patients reported a stinging during catheterization but no pain during dilatation. Mild hematuria happened immediately after the dilatation. False passages happened in four patients and two patients required five redilatations.

The results of our experience suggest that balloon catheter dilatation is a reliable alternative for treatment of urethral strictures.

\section{SPACE 1560}

Psoas and lipsoas abscess: percutaneous drainage

A. Garcia Suarez (Presenter), J. L. Perez Picouto, M. Pastrana Ledesma, A. Bueno Horcajadas, R. Perez Aranguena, F. Pena Felipe, M. Hernandez Mora; Madrid

Percutaneous drainage of abdominal and retroperitoneal abscesses has been established as a viable if not preferable alternative to surgical intervention. Their efficacy of percutaneous drainage of psoas abscess was assessed in 16 patients. In six patients the abscess was secondary to renal and perinephric abscesses, four secondary to vertebral osteomyelitis, one to diverticulitis, one to perfored intestinal neoplasia, and four idiopatic.

Unlike intraperitoneal abscesses, psoas abscesses tend to have internal septae, this tendency for loculation required multiple catheters. The process of percutaneous drainage was monitored with computed tomography and fluoroscopy. The method eradicated the abscess in 14 patients and was palliative in two patients who were subsequently cured by surgery.

Two minor complications (bacteriaemias) were attributed to the percutaneous drainage procedure. None major complications.

The mean time for hospitalization was reduced with percutaneous drainage. The method is recommended for initial management of psoas abscesses.

\section{SPACE 1561}

Stenosis of penile and bulbar urethra: diagnosis by ultrasound V. Garcia-Medina (Presenter), J. D. Berna, J. Llerena, J. Garcia-Medina, J. Rico, M. Tomás, J. Guirao, J. L. Genovés; Murcia

We evaluated the role of urethral sonography as an alternative technique to conventional retrograde and or voiding urethrography in the diagnosis of penile and bulbar urethral stenosis.

Methods and Materials: Sonography examination of the urethra was performed using a $7.5 \mathrm{MHz}$ and $10 \mathrm{MHz}$ transducer that is applied over the penis. The lumen of the urethra was well seen after the instillation of $6 \mathrm{cc}$ of sterile saline within the distal portion of the urethra.

Results: Fourteen patients with ages ranging between 35 and 68 years underwent urethral sonography for suspected stenosis. Complete correlation between the results of urethral sonography and conventional urethrography was found among the 10 patients with stenosis in the penile urethra. Three of four $(75 \%)$ of the bulbar stenosis were correctly diagnoses by urethral sonography. There were no complications related to the procedure.

\section{SPACE 1562}

The balloon catheter urethroplasty: 22 cases

V. Garcia-Medina (Presenter), J. Garcia-Medina, J. D. Berna, J. Guirao, J. L. Genovés, M. Alcaraz, P. Alemán, J. L. Rico; Murcia

In this exhibit, we evaluated the balloon prostatic dilatation in a group of 22 patients with hypertrophy of prostatic glands.

Methods and Materials: A total of 22 procedures of balloon dilatation were performed under fluoroscopic guidance. All of patients had urethral stenosis for prostatic benign hypertrophy without medium lobe prostatic.

Results: Follow-up evaluations showed voiding improved in 18 patients at 6 months, and 12 at 1 year. (Clinical, urodynamic, and radiological). There were no complications during and after procedure.

\section{SPACE 1563}

Utility of pulsed Doppler (PD) in the follow-up of steroid resistant acute vascular kidney graft rejection (AVRG) treated with OK-T3 monoclonal antibody or antilymphocyte serum (ALG)

R. Gilabert (Presenter), C. Bru, J.M. Campistol, L. Bianchi, R. Vilana; Barcelona

AVGR is a severe form of acute graft rejection that requires early diagnosis and treatment. The aim of the study was to determine prospectively the utility of PD in monitoring graft response to OK-T3 or ALG in severe (steroid resistant) AVGR.

The study group included 15 patients with AVGR, documented by renal biopsy, treated with OK-T3 (8 p.) or ALG (7 p.) who were sequentially studied with PD. PD spectrum was obtained at all arterial renal levels and was quantified by the Resistive Index (RI). PD studies were done 48 hours postgrafting, in graft dysfunction episodes and in monitoring graft response to rejection treatment. All AVGR episodes coursed with abnormal PD studies: RI range : $0.89-1$, with decreased, absent or reversal diastolic blood flow. Normalization of diastolic blood flow was documented prior to the decrease of serum creatinine in 12 patients who recovered from rejection. No RI improvement was found in the remaining 3 patients who did not recover their graft function. Conclusions: PD was a useful tool in the follow-up of AVGR. Prompt recovery of PD spectrum suggests a good prognosis.

\section{SPACE 1564}

\section{Extracorporal MR imaging of renal carcinomas}
O. Hélénon
(Presenter).
A. Denys,
M. Souissi,
Y. Chrétien

M. Mendelsberg, D. Droz, B. Dufour, J.F. Moreau; Paris

Purpose: To assess the value of ex vivo MRI in extracorporal conservative surgery of small renal carcinomas with autotransplantation.

Methods and Materials: After an enlarged nephrectomy, 21 kidneys perfused with a cold sterile Collins solution were imaged. We performed $\mathrm{Tl}$ and T2-weighted SE sequences and Gradient echo sequences (TR 300-400 ms, TE $30 \mathrm{~ms}$, flip angle $15^{\circ}$ ) with a surface coil, using $5 \mathrm{~mm}$ slice thickness. MR findings were correlated to in vivo MRI and with macroscopic and histologic results.

Results: Ex vivo MRI permitted the detection of 2 other small neoplastic lesions less than $5 \mathrm{~mm}$ in size and a small angiomyolipoma of $5 \mathrm{~mm}$. The results demontrated also the value of T2-weighted images in the diagnosis of small renal tumors. Regarding the peripheral capsule, they showed a poor correlation between MR findings and histopathologic results.

Conclusions: Ex vivo MRI seems to be able to improve the effectiveness of extracorporal partial nephrectomy of small renal tumors avoiding uncomplete conservative surgery in case of another intrarenal neoplastic lesion.

\section{SPACE 1565}

Interest of ultrafast CT (UFCT) In renal disease

S. Flais, A. Hernigou (Presenter), R. Kaddouche, G. Prijent, T. C. Gaux, M. C. Plainfosse; Paris

Purpose: Patterns of different vascular anomalies of kidney are illustrated. Methods and Materials: - UFCT with 17 slices in 25 seconds allows a perfect analysis of vascular pedicle and kidney parenchyma during the cortical time. The flow program provides the possibility of studying the renal blood flow (4 levels $/ 20$ images per levels).

- 20 patients were studied for morphological anomalies and 15 patients 
underwent a flow study (transplanted kidney in 5 cases).

Results: - Repercussion of vascular disease on the parenchyma is reliably detectable better than on a conventional CT because of perfect opacification of the cortex: infarction and hypoperfusion ( 5 cases).

- Anomalies of the renal arteries are easily recognized, illustrated by aneurysms, dysplasia, traumatic fistula ( 2 cases), or ostium lesions in aortic diseases.

- The flow program allows a dynamic visualisation of tumor vascularisation and parenchymal hypoperfusion. Quantification of the renal bood flow is expected with this technique. Results about 8 patients are presented.

Conclusions: UFCT with perfect arterial bolus is a reliable technique for detection of vascular anomalies of the kidney, but more than with a conventional $\mathrm{CT}$ the method of injection has to be adapted to the presumed pathology to avoid pitfalls or false negatives.

\section{SPACE 1566}

Interpreting ultrasonic properties of normal renal tissues in terms of microscopic anatomy

Michael F. Insana (Presenter), Timothy J. Hall, Linda Harrison, Stanton J. Rosenthal, James L. Fishback, Samuel J. Dwyer III; Kansas City, KS

Purpose: To explain the echographic appearance of normal renal tissues in terms of the acoustic properties and microscopic anatomy.

Methods and Materials: Tissue characterization techniques and a low-resolution acoustic microscope $(<15 \mathrm{MHz}$ ) were used to measure speed of sound, attenuation and backscatter coefficients, and the average size and number density of scatterers for excised human and dog kidneys. Data were obtained between 1 and $15 \mathrm{MHz}$ in the medullary and cortical regions as a function of the angle between the transducer beam axis and tissue structures. Parametric images of acoustic parameters were obtained using clinical instrumentation for comparison with B-mode images.

Results: The attenuation and backscatter coefficient values exhibit a significant directional dependence, which is more pronounced at frequencies $>5 \mathrm{MHz}$. Both measurements increased when the transducer beam axis was oriented perpendicular to structures such as the medullary rays. Backscatter anisotrophy was highly correlated with scatterer number density; the scatterer size and speed of sound measurements were isotropic. The dominant scattering structures below $5 \mathrm{MHz}$ are the renal corpuscles. Above $5 \mathrm{MHz}$, scattering from renal tubules and blood vessels dominate, as indicated by greater anisotrophy and scatterer size measurements. Several of these methods may be used in vivo to identify anatomical changes associated with pathology.

\section{SPACE 1567}

Benign prostatic hyperplasia revealed by transrectal ultrasonography W. Kastlunger (Presenter), A. Furtschegger, W. Buchberger, T. Penz, D. Lungenschmid, F. Weber; Innsbruck

To obtain objective and reproducible results of prostatic size and echo pattern, patients must undergo transrectal ultrasonography. The painless, noninvasive examination can be done in a short time (about 10 minutes) without special treatment of the patient, except for cleaning the rectum by giving an enema at least 1 hour before examination. It is advisable to use a real-time, biplane, $5 \mathrm{MHz}$ transducer, which can be handled easily after being painless inserted into the cleaned rectum of the patient. During the last four years transrectal sonography was performed in $724 \mathrm{men}$, in each case the seminal vesicles and prostata were evaluated. The average age of examined men was about 71 years. In $73 \%(n=528)$ of examined patients the volume of the prostate increased up to $80 \mathrm{cc}$. Benign prostatic hyperplasia yields a heterogeneous sonographic pattern. In the sonogram stroma tissue is of echo-poor appearance, while glandular tissue show a dense, lamellar echo pattern. The sonographic results were confirmed by biopsy and clinical investigations. In $15 \%$ prostatic cancer was detected. Transrectal sonography of the prostate, for revealing benign prostatic hyperplasia is the most valuable noninvasive examination.

\section{SPACE 1568}

CT evaluation of renal cell carcinoma in patients with acquired renal cystic disease

Atsushi Kohno (Presenter), Toshiroh Hayano, Arimi Harasawa, Chisato Kawai, Akiko Narimatsu, Akiko Shigeta, Hiroshi Tohma; Tokyo

1. Purpose: It is well known that there is a high incidence of the occurrence of renal cell carcinoma (RCC) in patients with acquired renal cystic disease (ARCD) and it is considered to be difficult to diagnose RCC in ARCD by diagnostic imaging. No reports of the assessment of the diagnosis of RCC in $A R C D$ by $C T$ have been published. Accordingly, this study was undertaken to evaluate the capability of CT in the diagnosis of RCC in ARCD.

2. Methods and Materials: The subjects consisted of eight patients with pathologically proven RCC in addition to ARCD. Preoperatively, plain and contrast-enhanced CT was performed in all patients. Contrast enhancement was performed by the bolus injection technique. The CT findings were assessed retrospectively.

3. Results: The size of the RCCs ranged from 1.7 to $8 \mathrm{~cm}$. Four tumors were solid and 4 were cytic. All tumors demonstrated contrast enhancement within the solid portion or the cyst wall, though the degree of enhancement tended to be less than that of tumors in normal kidneys.

4. Conclusions: We conclude that contrast enhancement is mandatory to detect RCC in patients with ARCD, and if a mass shows contrast enhancement the diagnosis of RCC should be considered.

\section{SPACE 1569}

CT versus lymphangiography in assessment of lymph nodal metastases in cervical uterine cancer

Alfredo La Fianza (Presenter), Roberto Dore, Lorenzo Preda, Luciana Babilonti; Pavia

Purpose: The presence of lymph-nodal metastases in patients affected with cervical uterine cancer is of the upmost clinical relevance. We have evaluated accuracy of CT and lymphangiography in nodal staging in a retrospective series of 95 patients.

Methods: From January 1986 to April 198858 women were evaluated preoperatively by $C T$ and lymphangiography ( 49 subsequently submitted to radical hysterectomy, the remainder underwent percutaneous fine needle biopsy). Other 37 patients previously treated were evaluated by CT for clinically suspected relapse.

Results: In the first series accuracy was $91 \%$, sensitivity $88 \%$ and specificity $92.5 \%$ for lymphangiography; accuracy was $87.9 \%$, sensitivity $72.2 \%$ and specificity $95 \%$ for CT. In the second group CT accuracy was $94 \%$, sensitivity $100 \%$ and specificity $91 \%$

Conclusions: In early stages of the disease (I-II) lymphangiography gives better results than $\mathrm{CT}$; in advanced stages and relapse $\mathrm{CT}$ gives high accuracy. This information is useful for avoiding unnecessary surgical exploration. We retain that $\mathrm{CT}$ plays a role in staging cervical cancer, recognizes lymph-nodal metastases in advanced stages and in treated patients, in early stages CT is complementary to lymphangiography and needs percutaneous biopsy.

\section{SPACE 1570}

Scintigraphic demonstration of hernias associated with continuous ambulatory peritoneal dialysis

S. Meecham Jones, A. J. Liddicoat (Presenter), C. Evans; Cardiff

Continuous ambulatory peritoneal dialysis (CAPD) is an increasingly popular treatment option in chronic renal failure, particularly in elderly patients. Abdominal hernias are well recognized complications of CAPD, with a prevalence of around $15 \%$ in several series. Most of these hernias are not clinically apparent prior to the commencement of CAPD, the increased intraabdominal pressure being an important contributory cause. We have recently encountered five patients on CAPD with inguinal hernias. Because of the considerable edema due to leakage of dialysis fluid associated with hernias, it is often difficult or impossible to lateralize the side of the hernial sac, necessitating bilateral inguinal exploration. We describe a method of lateralizing the sides of origin of hernias by the simple procedure of adding technetium $99 \mathrm{~m}$-labelled DTPA to the dialysis fluid, and imaging over the groin area with the patient in the erect position. Representative images are demonstrated. Recently we have also demonstrated a leakage of dialysis fluid into the thorax causing recurrent effusion. Although this method has been previously described in the nephrology literature, it warrants wider recognition in view of the increasing number of patients undergoing CAPD.

\section{SPACE 1571}

The effects of transcatheteral ablations of tumorous kidneys with absolute Ethanol

Darko Ljubisavljević (Presenter), Slobodan Radojković, S.Lavrnić; Belgrade

We intend to show that tumorous kidney ablation with absolute ethanol is a very useful and safe procedure if performed by proper technique. We have been injecting absolute ethanol through the occlusive balloon catheter in the previously estimated amount in the last 7 years in more than 1000 patients. The procedure was performed either as a preoperative or a palliative (definite) one. We never had a single local or systemic complication. Peroperative blood loss after ablation was negligible, patients with distant metastases showed regression in number and dimensions of such metastases and their general condition significantly improved. 


\section{SPACE 1572}

Retrograde and volding urethral sonography: diagnostic evaluation of the anterior urethra

Jose M. Llerena (Presenter), Vicente Garcia-Medina, Juan D. Berna,Janis G. Letourneau; Minneapolis, MN

Purpose: To evaluate the role of retrograde and voiding urethral sonography as an alternative to conventional urethrography in the diagnostic evaluation of strictures within the anterior urethra.

Methods and Materials: Retrograde urethral sonography was performed after the instillation of $6 \mathrm{cc}$. of sterile saline within the distal portion of the penile urethra, with the aid of a $7.5 \mathrm{MHz}$ linear array transducer, that is applied over the penis. Voiding urethral sonography was also performed while the patient was voiding. In both instances the anechoic fluid within the urethral lumen gave us an excellent view of the penile and bulbar portions of the anterior urethra.

Results: Fourteen patients with a mean age of 51.5 years (range: 35 to 68 years) underwent retrograde and voiding urethral sonography for ruling out suspected anterior urethral stenosis. All the patients had conventional retrograde and voiding urethrography after urethral sonography was performed. Among the ten patients with stenosis in the penile portion of the anterior urethra, there was complete correlation between the findings of retrograde and voiding urethral sonography and the results observed with conventional urethrography. Three of four $(75 \%)$ of the bulbar stenosis were correctly diagnosed by urethral sonography. All the patients tolerated well urethral sonography with none complications. We conclude that urethral sonography is a technically easy, accurate, nonexpensive and safe procedure in the diagnostic evaluation of anterior urethral stenosis.

\section{SPACE 1573}

\section{Different metallic intraprostatic endoprostheses} Z. Marković (Presenter), S. Djordjević; Kragujevac

Metallic intraprostatic spirals of original construction as a functional urinary bladder-urethra stent we've performed in 170 patients with chronic urinary retention.

We used iron spirals covered by silver, gold, chrome as well as simple ones made of elementary iron. By means of echotomography and CT we analysed reaction between urin, prostatic tissue and spiral material. We found out following: spirals covered by rare metals cause minimal irritable reaction and can be successful replacement for retentional catheter. Elementary iron spirals cause local aseptic necrotic reaction, fibrosis and scaring of prostatic urethra, which constitute physiological stent and spiral is supposed to be taken out due to new borne recanalisation enabled normal miction!

\section{SPACE 1574}

\section{Lipomas of the spermatic cord}

A. Maroto (Presenter), J. Barceló, X.Serres, J.C. Vilanova, I. Lladó, F. Castañer, J. Ferrer; Girona

Purpose: Scrotal masses are common urologic problems and include a variety of benign nontesticular tumors such as lipomas, fibromas, dermoid cysts, lymphangiomas and others. We report four cases of lipomas of the spermatic cord presenting as scrotal masses.

Methods and Materials: We performed ultrasound and computed tomography (CT) in all four patients. Their ages ranged from 32 to 69 years. In all cases the prime clinical consideration didn't include the diagnostic of a lipoma and ultrasound exploration was indicated to rule out testicular malignancy.

Results: Sonography revealed in our patients large homogeneous, hyperechogenic paratesticular masses extending to the inguinal canal. CT confirmed the low absorption coefficient of the masses which represented fat.

Ultrasound and CT findings may suggest the correct diagnosis of a lipoma in cases dealing with a problematic scrotal mass.

\section{SPACE 1575}

\section{Prostatic transrectal ultrasonography}

T. S. Vizcaino (Presenter), J. Davila, S. Glez Agudo, S. Oliete, R. Wert, B. G. Castaño, P. Corral Benito, M. Rguez, C. Temprano; Madrid

Purpose: We present a brief introduction of the technique and advantage of transrectal ultrasonography, as well a selected image of the anatomy and pathology of the prostate gland.

We present the results and statistics of our experience.

Methods and Materials: We have studied 300 patients with TRUS. Diagnoses were confirmed by biopsy, surgery or clinical follow-up.

Results: Actually TRUS is the method of choice in prostatic pathology: Early diagnosis of malignancy, local staging, and follow-up treatment.

\section{SPACE 1576}

Transluminal angioplasty of renal transplant arterial stenoses Umman Sanlidilek (Presenter), Sadik Bilgiç, Gülden Sahin, Cemil Yağci, Meral Doğan, Neval Duman, Bülent Erbay, Uluhan Berk; Ankara

Purpose: Percutaneous transluminal renal angioplasty has been used with success in the management of renovascular hypertension. The purpose of this study was to determine the role of renal angioplasty in the treatment of renal transplant arterial stenoses.

Methods and Materials: Percutaneous transluminal renal angioplasty was performed in six renal transplant recipients aged 15-44 years. A contralateral femoral approach was selected in all patients. 4-5 F Balloon Angioplasty catheters (Schneider and Medi. tech.) 20 to $40 \mathrm{~mm}$ in length and 4 to $6 \mathrm{~mm}$ in diameter were used for dilatation. The patients were referred to angiography because of sudden onset of hypertension and/or signs of chronic rejection after renal transplantation. Significant renal artery stenosis (more than $50 \%$ of the original diameter) were determined on angiography. Transluminal angioplasty was attempted in the same session in three, and a week later in the remainder. Time for angioplasty was 2 to 24 months following transplantation. Results: Technical and early success were achieved in all patients. The stenotic segment was dilated up to 70 to $90 \%$ of original artery size. Restenosis occurred in one patient within three months and a second angioplasty was performed. No complications developed due to the procedure. Despite technical and early success, nephrectomy was performed in two patients with persisting chronic rejection signs and two patients underwent regular dialysis program for the same reasons developed in 2 to 6 months following the angioplasty. One patient remained symptomless for 10 months after the procedure. Transluminal renal angioplasty remains an alternative in the management of renal transplant arterial stenoses despite the relatively high late failure rates in this series.

\section{SPACE 1577}

CT-fluoroscopy (CTF): role in interventional urinary radiology C. Spreafico (Presenter), L. F. Frigerio, A. Marchiano', M. Milella, I. Spagnoli, F. Garbagnati, B. Damascelli; Milano

Purpose: To determine the value of a combined Computed Tomography-Fluoroscopy unit (CTF) in percutaneous interventional procedures on the urinary tract.

Methods and Materials: 7 percutaneous nephrostomies were performed and 3 ureteral stents positioned under this imaging modality. Centering, choice of access point on the skin and of access trajectory to the urinary tract took place under CT guidance with a real-time monitoring of the entire procedure under fluoroscopy.

The correct final position of stents and nephrostomy catheters was checked by CT.

Results: All the procedures were successfully performed. CT ensured optimal choice of percutaneous access in every case, while fluoroscopy monitored all phases in real time; something no other imaging modality can do. The whole procedure was performed on the CT scanner table, operators' exposure to radiation was low.

CTF increased the precision and speed of the maneuvers.

\section{SPACE 1578}

MR Imaging of the bladder invasion of pelvic malignancy Yukari Wakabayashi (Presenter), Mana Kuroda, Junichi Yokouchi, Kimihiko Abe, Saburou Amino; Tokyo

Purpose: The purpose of this study was to assess the potential of MR imaging in the evaluation of bladder invasion of malignant pelvic tumor.

Methods and Materials: Seventy-six patients, including rectal and sigmoid colon cancer $(n=22)$, uterine cancer $(n=25)$, malignant ovarian tumor $(n=28)$, and prostatic cancer $(n=1)$ were studied. Imaging was performed at $0.5 \mathrm{~T}$ Toshiba unit using $\mathrm{T} 1$ and $\mathrm{T} 2$ weighted spin echo sequences. Sagittal plane was obtained and coronal plane was added when necessary. Each image was studied as for intensity of the bladder wall, deformity of the bladder and existence of fat plane between the bladder and the tumor.

Results: Bladder invasions were proved surgically in eighteen patients and MR imaging correctly identified 17 of these cases. These cases had deformity of the bladder and only 1 case showed abnormal intensity of the bladder wall. There were 8 false positive cases and these cases had relatively dilatated and thin wall bladder.

Conclusions: MR imaging is a valuable tool in the diagnosis of the bladder invasion. And appropriate bladder distension is recommended. 


\section{SPACE 1579}

A new, non-surgical treatment for recurring urethral strictures: long-term temporary urethral stenting by Urocoil ${ }^{\infty}$

Daniel Yachia (Presenter), Mordechay Beyar; Hadera

Recurring urethral strictures are a great concern to us because their successful treatment is a challenge. Various dilatation techniques, internal urethrotomy or open urethroplasty techniques may partly resolve this disappointing problem. We are presenting a new, self-expanding and self-retaining stent made of medical grade stainless steel alloy (Urocoil ${ }^{\circledast}$ ). This stent was used in 55 patients who had recurrent strictures of various etiologies (traumatic, inflammatory or iatrogenic) and varying lengths $(0.5-8.0 \mathrm{~cm})$ along the urethra, from the external sphincter to the urethral meatus. The stent was inserted, under topical anesthesy of the urethra in reduced caliber under fluoroscopy after an adequate dilatation was made either by balloon dilatation or internal urethrotomy. This stent when released from its introducer to reach its maximal caliber $(26 / 32 \mathrm{~F})$ by radial self-expansion. The stents were left in the urethra up to 14 months and they could be removed without surgery. Mean follow-up is 14 months (range 3-24 months). All patients voided well and were continent after insertion of the stent. Distal migration occurred in 5 patients and repositioning was performed under fluoroscopy. During the followup period of 6-16 months (mean 11 months) after removal of the stent $88 \%$ of the patients remained free of recurrent stricture. In failed cases a new stent was inserted. No tissue ingrowth or stone formation was observed during the followup period in the stents.

This temporary stent which can be easily inserted as well as easily removed anytime is a very promising tool for the non-surgical treatment of recurring urethral stricture.

\section{SPACE 1580}

A new, self-expanding and self-retaining large caliber intraprostatic stent (Prostacoll $\left.{ }^{\infty}\right)$ in the treatment of prostatic obstruction

Daniel Yachia (Presenter), Mordechay Beyar; Hadera

Urethral catheterization is the mainstay in the treatment of complete prostatic obstruction until definitive treatment. Long-term catheterization is needed when patients are poor operative risk permanently or are recuperating after a cardiorespiratory or circulatory ailment waiting to become eligible for surgery. Despite the well known complications of long-term catheterization, the lack of an established non-surgical alternative for overcoming bladder outled obstructions leaves this manipulation as a widely used palliative treatment. Laterly intraprostatic stenting devices are entering our armamentarium as alternatives to indwelling catheterizations. Instead of the fixed caliber $(20 \mathrm{~F})$ intraurethral stents and the self-expanding large caliber stents which become incorporated into the prostatic urethral lining within 4-6 months becoming permanent implants, a new, large caliber $(26 / 32 \mathrm{~F})$, self-expanding and self-retaining, long-term but temporary intraprostatic stent (Prostacoil ${ }^{\oplus}$ ) was developed. The insertion of this new metallic stent is performed under fluoroscopy. This stent was used successfully during the last 2 years in 35 patients who needed indwelling catheterization who were not fit for surgery. Insertion of the stent under topical anesthesia using $2 \%$ Lidocaine jelly allowed $90 \%$ of the patients spontaneous voiding with good control of the external sphincter.

\section{SPACE 1581}

Perinephric and subcapsular renal hemorrhage: CT Imaging and therapeutic implication

D. Yaffe (Presenter), R. Zissin, V. Rathaus; Kfar Saba

The records of 6 patients with acute spontaneous perinephric or subcapsular hemorrhage were retrospectively reviewed for evaluation the value of computed tomography (CT) in its diagnosis and etiology. The initial CT study demonstrated the hemorrhage in all cases. An accurate diagnosis of the cause was established in 4 patients (two cases of perinephric liposarcoma one case of angiomyolipoma and a leaking abdominal aortic aneurism in the other) necessitated operation. No case of renal cell carcinoma, the most common cause, was found.

In the other two patients no distinct pathology was identified in the kidney or perirenal space, neither by CT nor by a subsequent angiography. These two patients were followed by CT for a period of up to three years showing resolution of the hemorrhage with no additional pathology. This conservative approach of serial, detailed C.T examinations is recently recommended in the radiological literature, preventing unnecessitated nephrectomy.
MRI of hydatidic disease (echinococciasis) in the musculoskeletal system: report of five cases

J. Aldoma (Presenter), E. Gomez, A. Capdevila, M. Moragas, P. Martinez, J. Gili, M. Baquero, M. P. Sanz; Barcelona

Hydatidic disease is a tissue infection caused by the larval stage of Echinococcus granulosus that produces cystic lesions involving the liver $(75 \%)$, the lungs $(25 \%)$ and occasionally other organs. The musculoskeletal system is affected in $1-2.4 \%$ of cases.

We have retrospectively evaluated with MRI 4 patients with histologically proven musculoskeletal echinococciasis ( 3 patients with recidivism of vertebral echinococciasis and 1 patient with echinococciasis synovitis) and 1 patient with a soft tissue mass in the left thigh.

MRI has been performed on a $1.5 \mathrm{~T}$ in 4 patients and on a $0.5 \mathrm{~T}$ in 1 patient. MRI ist not specific in hydatidic disease but has been useful in delineating the extension of the lesion for planning its surgical treatment in the 3 patients with vertebral echinococciasis and in the patient with a soft tissue mass in the thigh. In this last patient, the MR appearance of the image has been highly suggestive of hydatidic disease. MRI has not been useful in the echinococciasis synovitis, showing only a joint effusion.

\section{SPACE 1583}

Systemic sarcoidosis with skull involvement

A. Anguera (Presenter), X. Pruna, I.Segarra, A.Sanchez, V. Fabregas, X. Jori; Tarragona

Bone lesions in sarcoidosis are uncommon, and often their appears in chronic stage. Characteristically lesions are osteolytic without evidence of marginal sclerosis or periostal reaction and skull involvement are the rarest manifestations.

We report a case of skull sarcoidosis: a 54 year old woman was diagnosticated pulmonar sarcoidosis in 1989. In March 1990 she noted headache, lymph nodes in lateral cervical region and subcutaneous nodul in right front-parietal region. Skull radiographs and CT showed numerous erosive lesions of varying size in the calvarium and in jaw. A Ga and Tc99 scan showed numerous areas of increased uptake.

The interest of this case is to present iconography about skull sarcoidosis and the resolution with steroid treatment.

\section{SPACE 1584}

MR and $C T$ versus conventional $x$ ray plain film for the examen of solitary bone lesion

F. Aparisi (Presenter), I. Muguerza, J.L. Garci, J.Beltran, C. Cifrian, C. Espinosa; Valencia

The prognosis of bone lesions has been changed in the last ten years, result to the new techniques for bone tumors management. The radiologist's role now is the characterization of bone lesions, founding the extension, aggressivity and histological type. We have been studying 120 solitary bone lesions, with X Ray plain film, CT and MR.

The sensibility for to establish the limits were different. The index for MR are $95 \%$ while the CT are $55 \%$ and X Ray plain film $27 \%$. In the determination of the aggressivity the differences were less. The index is around $92 \%$ in all techniques. The new techniques don't provide new semiological information. For finding the histological type the CT was superior, with $36 \%$ of index, while the X Ray plain film are $22 \%$ and the MR $29 \%$. In the characterization of bone lesions the X Ray plain film has an important mission, although the correct treatment needs also to know the real extension. The new techniques give us this information.

\section{SPACE 1585}

MR imaging of the cervical spine in rheumatoid arthritis: interest of Gadolinium-DOTA IV administration

J. Assoun (Presenter), C. Poey, M. Pages, A. Roux, T. Billey, B. Fournie, J. J. Railhac; Toulouse

Purpose: Cervical spine involvement is frequent in rheumatoid arthritis (R.A.), but it is difficult to appreciate joint lesions except with roetgenograms. It is known that Gadolinium DOTA (Gd-DOTA) IV administration is taken up by inflamed synovium and we report the study with MRI and Gd-DOTA of 12 patients with cervical spine arthritis in R.A.

Methods: The 12 patients had classical of definite RA according to the ACR criteria, examined at our center in the years 1989 and 1990. All patients had clinical symptoms of cervical spine involvement (pain, stiffness, medullary or other neurological compressions). Plain radiographs are always performed (normal findings, erosions, subluxation, diastasis of $\mathrm{Cl}-\mathrm{C} 2$, inflammatory 
spondylodiscitis). MRI is realized on 0,5 Tesla Magniscan with a cervical coil in all patients: $\mathrm{Tl}$ weighted sequences before and after Gd-DOTA; T2 weighted sequences in sagittal and axial planes.

Results: MRI with Gd-DOTA IV administration seems very helpful in cervical spine of RA and allows better findings than $\mathrm{T} 2$ weighted sequences. Lesions are always most important in comparison with standard $X$ rays.

\section{SPACE 1586}

Magnetic resonance imaging of bone marrow in the staging of malignant lymphomas: correlation with bone marrow biopsy

J. Assoun (Presenter), C. Poey, M. Attal, P. Brousset, R. Aziza, P. Fajadet, P. Maquin, J. J. Railhac; Toulouse

Purpose: The evaluation of bone marrow status is usually obtained by bone marrow biopsy. However, small samples taken by iliac crest biopsies may not be representative of marrow elsewhere. MRI of the bone marrow has been shown to be a sensitive method for detecting a variety of marrow diseases and may be able to detect lymphomatous involvement.

Methods: To correlate magnetic resonance imaging (MRI) with bone marrow histology, a prospective clinical study was performed in 43 patients with malignant lymphomas. 8 patients with Hodgkin's lymphoma and 35 patients with non Hodgkin's lymphoma. Images were obtained of the proximal femora, pelvic and vertebral marrow with a 0,5 tesla MR system using a Tl-weighted spin echo sequence. MRI and unilateral iliac crest biopsies were performed before initiation of therapy.

Results: In all except five cases, results of MRI and bone marrow histology were concordant in three patients with low grade non Hodgkin's lymphoma (NHL), bone marrow histology revealed malignant infiltration without MRI abnormalities. Two patients with high grade NHL had marrow lesions detected by MRI that were not detected on initial bone marrow biopsies.

\section{SPACE 1587}

Popliteal masses in hemodialysis patients: the role of diagnostic imaging

B. Balbi (Presenter), L.Baldrati, A. Rocchi, R. Bonsanto, M. Mughetti, D. Docci, C. Capponcini, C. Feletti; Cesena

Popliteal masses have recently been described as a possible manifestation of dialysis-related amyloidosis (DRA). The aim of the present study was to evaluate the role of diagnostic imaging in detecting popliteal masses in hemodialysis patients.

By means of ultrasonography (US), popliteal cysts have been demonstrated in 12 (bilateral in 9) out of 37 patients $(32.4 \%$ ) dialyzed for more than 60 months (range 60-225, mean $122 \pm 48$ ). In the 3 patients who were investigated by CT, cysts could be demonstrated to communicate with the joint cavity (Baker's cysts). In 1 case, immunocytochemical analysis showed diffuse 32 Microglobulin-amyloid deposition within the synovial wall of the surgically removed cyst. The 8 patients with largest cysts (medium size $>20 \mathrm{~mm}$ ) suffered some of the major features of DRA: carpal tunnel syndrome ( 7 cases), destructive arthropathy (5 cases), shoulder and carpal cystic radiolucencies ( 6 and 5 cases). In 1 patient, in association to Baker's cyst, MR showed two small extraarticular, subcutaneous masses (probably amyloid-tumors).

These findings document the high prevalence of popliteal masses among chronic hemodialysis patients, often associated to DRA; the importance of US to detect popliteal masses must be stressed.

\section{SPACE 1588}

\section{Dialysis arthropathy: radiological and clinical findings}

B. Balbi (Presenter), A. Rocchi, L. Baldrati, R. Bonsanto, M. Mughetti, D. Docci, C. Capponcini, C. Feletti; Cesena

Many long-time (>60 months) hemodialysis patients develop a severe osteoarticular disease, termed "dialysis arthropathy", which is characterized by the deposition in bone and synovia of a new type of amyloid composed predominantly of $\beta 2$-microglobulin. In the present study, 45 patients $(25$ males, 20 females: age $56.7 \pm 12.6$ years), undergoing chronic hemodialysis for 60-225 months (mean 115 \pm 49 ), were screened for dialysis arthropathy by means of clinical examination and radiological investigations (standard radiography and computed tomography).

Twenty-four patients $(53.3 \%)$ had radiographic evidence of dialysis arthropathy: geodes (shoulder 18 cases; wrist, 18; hips, 6; and knees, 2) and/or destructive arthropathies (cervical spine, 15 cases; dorsolumbar spine, 2: hands, 2: and hips, 1). Within 24 months, these lesions were found to progress slowly in the majority of cases. Patients with dialysis arthropathy had been maintained on hemodialysis for longer periods of time than these without it $(\mathrm{p}<0.001)$, and showed a significantly higher incidence of both carpal tunnel syndrome $(\mathrm{p}<0.0005)$ and shoulder pain $(\mathrm{p}<0.01)$.

Our findings confirm the high prevalence and clinical importance of dialysis arthropathy in long-term hemodialysis patients and the usefulness of diagnostic imaging in screening such patients for these lesions.

\section{SPACE 1589}

Evaluation of "impingement point" using MRI in the degenerative painful shoulder

A. Barile (Presenter), C. Masciocchi, E. Fascetti, R. Passariello; L'Aquila

The purpose of this study is to illustrate our preliminary experience with MRI in the evaluation of "impingement point" in the degenerative painful shoulder. From January ' 88 to December ' 90,100 patients (with clinical findings of rotator cuff abnormalities) selected by 850 cases of shoulder MRI examinations, were considered. MRI was performed with a $0.5 \mathrm{~T}$ superconductive (Esatom MR 5000) and with a 0.2 permanent (Esatom PM 4000) system. In all cases a "dedicated" surface coil was used. Transverse, sagittal and coronal oblique $\mathrm{Tl}$ and $\mathrm{T} 2$-weighted images first using spin-echo and gradient-echo sequences were used. All scans were considered of diagnostic quality. MRI showed in 9 patients impingement syndrome due to isolated subacromial bursitis (type I), type IIa (tendinitis) in 55 patients, type IIb (small or partial rotator cuff tear) in 12 patients and type III changes (complete rupture of the rotator cuff) in 24 cases. In conclusion the results of this experience demonstrate that MRI has considerable potential in the evaluation of patients with shoulder pain.

\section{SPACE 1590}

Atlas of serendipitous findings of soft-tissue abnormalities discovered on bone scintigraphy

Kirkman G. Baxter (Presenter), David F. Preston, Ralph G. Robinson, Mark D. Murphey; Kansas City, KS

Purpose: A wide variety of soft-tissue diseases and conditions may be demonstrated serendipitously on Tc-99m phosphate bone scans. The appreciation of these extraosseous soft-tissue accumulations of phosphate often adds to the diagnostic quality of the bone scan and improves its clinical utility.

Methods and Materials: This exhibit will present a wide spectrum of abnormal studies with extraskeletal phosphate deposits in both benign and malignant conditions. Abnormal soft tissue findings will be demonstrated for nearly every major abdominal organ. Radiographic and pathologic correlations will be presented to accompany the scintigraphic findings. The subtlety and importance of these findings will be demonstrated through a series of case presentations.

Results: The exhibit demonstrates the importance of soft-tissue abnormalities on the routine bone scan and directs the attention of the physician to these important findings.

\section{SPACE 1591}

Ultrasonographic examination of muscle hematomas in hemophiliacs I. Castells Ferrer (Presenter), R. Dominguez Oronoz, L. Casas Gomila, J. Capellades, E. Castellà, C. Altisent; Barcelona

Spontaneous hemorrhage into soft tissues is not rare in patients with hemophilia. Ultrasonographic examination is of great value in diagnosis of intramuscular and subcutaneous bleeding. Although such bleeding may be clinically apparent, the exact location is difficult to establish.

The muscle bleeding may be interstitial, with increased size of muscle and dislocated intramuscular fasciculi, or a localized lesion, anechoic or complex with regions of hyperechoic structures indicating blood clot formation.

Sonography was performed on 21 hemophilic patients with suspected intramuscular bleeding, within 24-48 hours after the onset of the symptoms. Commercially available real time sonographic equipment was used with $5-\mathrm{MHz}$ linear array transducer.

In 18 of the 21 patients studied, sonography confirmed the presence of 24 hematomas. 19 hematomas were intramuscular, 10 interstitial and in the other 9 the muscle bleeding was a localized lesion. 4 were insubcutaneous tissue and the other one was a hemophilic pseudotumor of the pelvis.

\section{SPACE 1592}

Familial chondrocalcinosis in the Catalan area of Osona (Barcelona Spain)

J. A. Clavero Torrent (Presenter), J. A. Garcia Conesa, J. Gispert Aceña R. Sanmarti Sala, D. Pañella Mora, F. Autonell Reixach; Barcelona

1. We present the radiological findings of 4 nonconsanguineous families, resident in Osona, affected by hereditary chondrocalcinosi (HACC).

2. During a period of 24 months, 41 cases of articular chondrocalcinosis (ACC) were diagnosed in the General Hospital of Vic. Of these cases, 4 began their symptomatology before the patients were 40 years old. A radiologic study was performed on the families of these 4 patients. This study consisted of, at least, $x$-ray films on the knees, pelvis, wrists and hands which showed that some others members of each family were also affected. Radiological pattern of disease was heterogeneous, with a poliarticular involvement and destructive arthropathy in one family, oligoarticular involvement in two families and the 
coexistence of the two forms in the other family.

3. Classically, the HACC has been considered more infrequent than the ACC of idiopathic type or the ACC related to metabolic illnesses. The description of these $\mathbf{4}$ families is the first one of the HACC type in Catalonia and it brings the following considerations:

a) that early onset of the ACC symptomatology is suggestive of the hereditary type (HACC).

b) The different expressivity of HACC among members of the same family.

\section{SPACE 1593}

Examination of cruciate ligaments of the knee by ultrasonography and computer tomography

Z. Csókási (Presenter), I. Bali; Szeged

The cruciate ligaments have an important role in the statics of knee. However the injury of cruciate ligaments is frequent and the operation can be favourable. The early diagnosis enhance the chance of recovery. The ligaments can be represent by UH and CT. The authors examined 48 patients with suspicion of rupture of cruciate ligament. The examination were performed by Acuson 128 with $5 \mathrm{MHz}$ linear transducer and Somatom DRG machine. During both examinations they tried to represent the anatomical structures as well as direct and indirect signs of rupture. According to their experiences the normal situation can be represented well. In many cases during US examinations indirect signs only refer to rupture. The authors have found that the locality and the degree of rupture can be determined by CT. The US can be used to exclude the rupture, however the CT examination is necessary for a precise valuation.

\section{SPACE 1594}

Comparison of bone scintlgraphy and MRI in detection of bone metastases of the spine

R. W. Dalicho (Presenter), E. G. Eising, Th. Lehnen; Recklinghausen

In the posttherapeutic care of tumor patients with bone metastases, the use of MRI would be increase in future, if clinical status differs from conventional radiographs and/or bone scintigraphy in evaluating the metastatic extension or complications (e.g. compression of the spinal cord of non-bony metastatic specimen).

Because of the spine can be cleared up sufficiently by all of these diagnostic procedures, we have performed bone scintigraphy and MRI of about 40 tumor patients to compare these three imaging modalities.

\section{SPACE 1595}

Radionuclide assessment of the results from correction of blood supply of the femur head

E. Baltov, M. Dontchev (Presenter); Pleven

Authors are estimating the vitality of the bone transplant using noninvasive methods of nuclear medicine.

52 patients were followed by $\mathrm{Tc}^{99 \mathrm{mC}}$ scintigraphy. The operations were performed according to the methods of: Vanable and Stuck (46 patients), Ficat ( 2 patients), Pho ( 2 patients) and authors' own method ( 2 patients). The results were evaluated by comparison of the level of fixation of $\mathrm{Tc}^{99 \mathrm{mC}}$ in the area of operated and nonoperated side and also the donor area in some suitable patients.

The possibilities of the methods used are argued.

\section{SPACE 1596}

Radionuclide and sonography control of the small joint prostheses success

M. Dontchev (Presenter), D. Vassilev, E. Baltov, H. Todorov; Pleven

$\mathrm{Tc}^{99 \mathrm{mC}}$ scintigraphy and ultrasonography were used for the success assessment of small joints endoprostheses.

42 endoprostheses were placed in the same number of patients. They were followed by scintigraphy and sonography. The patients were operated on due to RA. TBC, aseptic necrosis and traumas. As it is well known in these patients sometimes the placement of small joint prostheses is nessessary. These endoprostheses are mainly under torsion pressures which could lead to the prostheses loosening. The authors will give their conclusions about the advantages and disadvantages of both control methods used.

\section{SPACE 1597}

Ultra low field MRI $(0.064 \mathrm{~T})$ of the knee and arthroscopy: a double blinded study in $\mathbf{5 0}$ patients

Massimiliano D'Erme (Presenter), Michele Rossi, Massimo Ventura, Piero Maceroni, Marco Pasquali Lasagni; Latina

Purpose: To prove the possibility of ultra low field MRI (ULFMRI) to perform diagnostic exams of the knee in comparison with arthroscopy.

Methods and Materials: From June 1989 to February 1991 more than 150 exams of the knee have been performed with a $0.064 \mathrm{~T}$ unit (TOSHIBA ACCESS), having obtained with 3D partial hip angle (3dPF) sequences excellent results in term of quality of imaging and diagnostic capability. To prove these impressions we have decided to perform a double blinded prospective study based on 50 consecutive patients examinated both with MRI and arthroscopy.

With MRI we are using the following imaging parameters:

- Coronal 3dPF (TR 68ms, Te 24ms, FA45, $164.5 \mathrm{~mm}$ slices in $4^{\prime} 25^{\prime \prime}$ )

- Sagittal 3dPF (TR 68ms, TE 24ms, FA45, $323.5 \mathrm{~mm}$ slices in $9^{\prime}$ )

- Sagittal 3dPF (TR $110 \mathrm{~ms}$, TE 40ms, FA12, $323.5 \mathrm{~mm}$ slices in $11^{\prime}$ ).

If need, for a better evaluation of the anterior cruciate (LCA), a transaxial $2.2 \mathrm{~mm} \mathrm{3dPF}$ or a Sagittal $5 \mathrm{~mm} \mathrm{SE}$ is used.

The day after the MRI examination, an arthroscopy is performed.

Results: The early findings ( $15 / 50$ patients) from ULFMRI and arthroscopy agree in $93 \%$ of the time on meniscal tears, in $75 \%$ on LCA lesions and $87 \%$ on grade III and IV chondritis.

Conclusions: These preliminary results seem to confirm the possibility of ULFMRI to be used in the study of the knee, being able to perform diagnostic study in short time, at low price and with results comparable to what obtained at higher field strength.

\section{SPACE 1598}

The significance of the streakes of Harris in the lower limbs fractures: resulting from a study on 53 cases

G. Ferrozzi (Presenter), F. Ferrozzi, F. Draghi, A. Morandi; Piacenza

Metabolic deficiency of various kinds, acute or chronic deseases and physical stress especially due to fractures may give rise to the formation, in the metaphysis, of same transverse thickening lines known as "Harris's streakes". The Authors have selected 53 cases concerning lower limbs fractures in young people. These cases were accompanied by X-Ray photograph of the injured segment at a significant space of time from trauma. The importance of the discovery of these thickening lines, which appear in a high percentage of cases, is being discussed also according to a medico-legal point of view, as these streakes clearly show the time in which the fracture occurred and consequently the cause of an eventual hypermeter of the injured limb.

\section{SPACE 1599}

\section{Arthrography of the wrist in carpal injuries}

B. Fritsch, F. Boudard, J. M. Lamoureux, Ph. Fournot (Presenter); Toulon

AIM: Preoperative evaluation of ligament disruptions.

Methods: Continuous digital recording following injection subtraction techniques and video-images. 110 cases.

Results: 110 cases. Staging of ligament disruptions in carpal injuries.

\section{SPACE 1600}

\section{Utility of CT in femoro-patellar disturbances in athletes}

A. Framiñan (Presenter), J. Escalada, E.M. Sanchez, J. Usabiaga, J.J. Garcia-Nieto, L. Sanchez; Salamanca

Purpose: Femoropatellar wear due to maltracking is one of the most frequent causes of pain, unrelated to trauma, in the knees of sportsmen. In patients with this pathology, conventional axial radiology has traditionally proved to be negative. Thus we were prompted to make use of $C T$ in order to evaluate the imbalance in extension, at a few degrees or flexion.

Methods and Materials: 22 athletes, 17 females, in the last 4 years. Standard radiological projections, axial projection at $30^{\circ}$ with external rotation of the tibia, and a CT scan at $20^{\circ}, 10^{\circ}$, and $0^{\circ}$ flexion both with and without contraction of the cuadriceps, were taken in all the patients. The orthopaedic treatment failed in 22 cases, therefore they had to undergo surgery. Postoperatory CT control was carried out after 3 months.

Results: The utility of CT in the athletes studied here, is above all useful for realignement or correction obtained by the treatment applied rather than a strict method of diagnosis. 


\section{SPACE 1601}

\section{Tuberculous dactylitis}

M. Gil (Presenter), E. Andia, A. Sanchez, V. Fabregas, F. Jori; Barcelona

Tuberculous dactylitis is a rare musculoskeletal infection that mainly affects children. The radiologic features are soft tissue swelling, periostitis with bone expansion and cortical destruction, diffuse uniform infiltration and localized osteitis. A positive chest radiograph for tuberculosis is more common in children than in adults.

We report a case of a 24 year old woman who had been treated of pulmonar tuberculosis several years ago. She was admitted at our hospital and diagnosticated of miliary tuberculosis. Physical examination revealed circumscribed swelling over the palm of the right hand discharging purulent material. Roentgenograms showed localized osteitis of the distal phalanx of the third and fourth finger of the right hand. Chest radiograph was positive for tuberculosis. Cultures from the draining material of the hand and from bronchial secretions grew Mycobacterium Tuberculosis. The patient underwent a combined medical and surgical treatment and had a good evolution.

\section{SPACE 1602}

Uitrasound in the evaluation of subcutaneous lipoma

I. Gillard (Presenter), S. A. Sintzoff jr., P. A. Gevenois, D. Van Gansbeke, A. Van Oost, I. Salmon, J. Struyven; Bruxelles

Purpose: The purpose of the study is to evaluate the performance of ultrasound in the evaluation of suspected subcutaneous lipoma.

Methods: 21 patients were prospectively evaluated with an Acuson 128 computed tomography unit equipped with a $5 \mathrm{MHz}$ transducer. The following parameters were studied: the size, the margins, the echogenicity towards the surrounding fatty tissue and the presence of distal sound enhancement or acoustic shadowing. MRI was performed in 4 patients with large masses with ill-defined margins. Surgery and pathological examination were performed in all patients.

Results: The histological diagnosis included 1 epidermoid cyst, 1 liposarcoma and 19 benign lipomas. The patients have been classified in 3 different groups on the basis of sonographic features. The first group includes the 4 patients who underwent MRI: at ultrasound, all had large heterogeneous tumor with posterior acoustic shadowing or ill-defined margins. MRI demonstrated typical lipoma in 3 cases as proven by the pathological examination. The last case did not exhibit a lipoma pattern on MRI and corresponded to the liposarcoma. The second group included 3 patients wiht hyperechoic small subcutaneous tumors. All were confirmed to be lipomas. The last group included 14 patients with hypoechoic heterogeneous well-defined masses. 6 masses had distal acoustic enhancement, 5 were proven lipomas and one an epidermoid cyst that was indistinguishable from the other masses on the basis of sonographic criteria. The other 8 masses exhibited no acoustic enhancement and were all proven to be lipomas.

Conclusions: Ultrasound is a useful method in the evaluation of small subcutaneous lipomas. For large masses, especially when the margins are ill-defined, ultrasound cannot make the differential diagnosis with other soft tissue tumors and MRI may be helpful.

\section{SPACE 1603}

Radiologic finding in avulsion fractures and their mimics

A. Gonzalez Agudo (Presenter), D. Gomez Santos, S. Fdez Zapardiel, F. Maria Salgado, R. Wert Ortega, S. Oliete Sempere; Mostoles

An avulsion fracture is a failure of bone resulting from the application of tensile force through a musculoskeletal unit or a ligament the weakness is commonly a reflection of the young unfused apophysis or a weak osteopenic bone of any cause.

The panel attempt to study the different radiographic manifestations of these injuries helps the physician to arrive at an accurate diagnosis and leads to prompt and appropriate treatment for the patient.

Understanding the mechanisms of injury knowledge of the specific tendons or ligaments involved.

To show the many normal variants, commonly presents in the ossification center, same localizations this type of fractures, waking easy the differential diagnosis.

Methods and Materials: We review all the avulsion diagnosed in 150.000 traumatic injuries attended in our Hospital between March 1983 and June 1990.

We show a broad spectrum of avulsion fractures of the skeletal system. Some of then very incommon showing in some cases the radiological outcome and the normal variants that can lead to diagnostic errors.

\section{SPACE 1604}

\section{Cystic rheumatold arthritis}

F. M. Gubler (Presenter), M. Maas, P. F. Dijkstra; Amsterdam

Our purpose was to describe a cystic form of rheumatoid arthritis (RA). Methods: Patients were selected with peri-articular intraosseous cysts, without erosions, at their radiographs. The radiographs and medical histories were reviewed.

Results: We found a cystic form of RA in $9 \%$ of 220 consecutive patients with RA. The mean follow-up time was $10(1-39)$ years. The cysts were distributed symmetrically, often located at the proximal side of the joints and predominantly found around the PIP, MCP, and wrist joints of the hands, and the IP-1 and MTP joints of the feet. CT showed a peripheral intraosseous cyst localisation. MRI showed that the cysts may contain fluid, inflamed synovia, or both. Radiological and clinical progression, during the course of the cystic RA, was far less compared to classic RA.

Conclusions:

- Cysts can be an important diagnostic feature, and a supplement to the ARA-criteria, in the diagnosis of RA.

- Patients with cystic RA may have a relative benign course of their disease. In this exhibit radiographs, radionuclide bone-scans, CT and MRI images will illustrate the presentation and course of cystic RA.

\section{SPACE 1605}

Limited usefulness of traction-compression films in radiographic diagnosis of lumbar segmental instability: comparison with flexionextension films

Marja Hakola (Presenter), Hannu Manninen, Seppo Soimakallio; Kuopio

Lateral radiography during traction-compression provocation has recently been reported as an accurate method of radiographic diagnosis of segmental instability of the lumbar spine, especially in patients with spondylolisthesis and -lysis. The purpose of this study was to compare traction-compression (t.c.) method with flexion-extension (f.e.) method for detection of segmental instability.

Methods and Materials: F.e. and t.c. radiographs of 306 patients with clinically suspected lumbar spinal instability were analysed. The mean age of the patients was 43 years. In f.e. and t.c. films $4 \mathrm{~mm}$ or more forward/backward displacement and in f.e. films also excessive angular motion or signs of axial rotation of individual motion segments revealed instability. The chi-square test and statistic kappa were used as statistic method.

Results: Up to $92 \%(87 / 94)$ of the instability findings could be seen only on f.e. films, while $3 \%(3 / 94)$ of the instability cases could be diagnosed only on the t.c. films. In $4 \%(4 / 94)$ of the instability cases, signs of instability could be seen with both methods. For diagnosis of instability f.e. and t.c. films were not significantly interrelated, intertechnique agreement by statistic kappa remained fair. The usefulness of t.c. films in diagnosis of lumbar spinal instability is evidently very limited.

\section{SPACE 1606}

Dual-energy X-ray absorptiometry (DXA): extended clinical applications Franz Kainberger (Presenter), G. Seidl, P. Hübsch, A.Sterk, S. Trattnig, P. Pietschmann, A. Vana; Vienna

Purpose: To illustrate the broadened spectrum of clinical applications of modern dual-energy projection methods (DXA).

Methods: Using body scanners with a X-ray source (Hologic QDR-1000 ${ }^{\mathrm{TM}}$, Norland-XR 26) bone mineral density (BMD) and content (BMC) were measured in 346 adult patients: 277 patients with disturbance in calcium metabolism (primary hyperparathyroidism, renal osteodystrophy) or with diabetes mellitus, 29 patients with localized abnormality of calcium content (metastasis, Sudeck's atrophy, or after implantation of hip prosthesis), and 40 patients having performed abnormal physical exercise (immobilization, sporting activities). Correlation was done between measurements before and after treatment and/or final clinical outcome.

Results: DXA, although providing integral measurements, is suitable to quantify calcium content with high spatial resolution and low radiation exposure. In renal osteodystrophy, BMD values improved significantly after renal transplantation (total body-BMC from $2501 \pm 437 \mathrm{~g}$ to $2582 \pm 411 \mathrm{~g}$, $p=0,105$ within 3-8 months). Periprosthetic BMD measurements are not influenced by metallic artifacts. Significant changes could be documented in handball players or after physical therapy (lumbar BMD from $0.838 \pm 211 \mathrm{~g} / \mathrm{cm}$ to $0.908 \pm 345 \mathrm{~g} / \mathrm{cm}$ ).

Conclusions: DXA is a useful adjunct in grading not only osteoporosis but also various generalized and localized abnormalities of calcium content of the bone. 


\section{SPACE 1607}

Impact of dynamic axial forces on lumbar spine stability M. Amann, J. Kramer (Presenter), G. Rauscher, H. Imhof; Vienna

Purpose: To study the influence of a cyclically changing stress on lumbar spine stability.

Methods: Seven isolated lumbar vertebrae and 17 motion segments consisting of two adjacent lumbar vertebrae connected by their intervertebral disc were subjected to various modes of compression in vitro, thus simulating a narrow spectrum of everyday stresses on the lumbar spine. Magnification radiography and bone densitometry were done before and after testing. Preparations were halved sagitally and examined histologically for morphology of possibly present fractures. Stress testing consisted of $I \mathrm{kN}$ preload for 1 hour with a first cycle of $80 \mathrm{~ms}, 1 \mathrm{kN}$ impulses and then a second cycle of $80 \mathrm{~ms}, 2 \mathrm{kN}$ impulses superimposed. Dynamic stiffness was determined by sinusoidal $0.5 \mathrm{kN}$ impulses at $12.5 \mathrm{~Hz}$ superimposed on a $1 \mathrm{kN}$ preload.

Results: The static deformation of an isolated vertebra after the first cycle was $70 \%$ of that experienced by a motion segment, after the second cycle there was no significant difference. Dynamic deformation of an isolated vertebra was only $45 \%$ compared to a motion segment. Half of the preparations $(4 / 7,10 / 17)$ showed microfractures in their bony end plate.

Conclusions: 1. Static measurements do not sufficiently demonstrate the amount of deformity of a motion segment.

2. In a motion segment the disc is responsible to $55 \%$ for the elastic behaviour. 3. Elasticity loss of the disc results in a higher risk for vertebral microfractures.

\section{SPACE 1608}

Rheumatoid arthritis of the hand and wrist: unenhanced versus Gadopentetate-Dimeglumine enhanced MR Imaging

Philipp Lang (Presenter), Michael Stevens, Kenneth Sack, Sharmila Majumdar, Martin Vahlensieck, Ulrich Speck, Harry K. Genant; San Francisco, CA

Purpose: Conventional radiography is limited in delineating inflammatory soft-tissue changes in rheumatoid arthritis (RA). We evaluated the use of unenhanced and gadopentetate-dimeglumine (Gd-DTPA) enhanced MR imaging in 15 RA patients with wrist involvement.

Methods: AP -, lateral, and oblique radiographs of the wrist were obtained in all patients. MR imaging was performed before and after i.v. administration of $0.1 \mathrm{mmol} / \mathrm{kg}$ body weight Gd-DTPA (Magnevist ${ }^{\oplus}$, Schering, Berlin) (Precontrast: spinecho, $\mathrm{TR}=600 \mathrm{msec}, \mathrm{TE}=20 \mathrm{msec}, \mathrm{T} 2-\mathrm{WI}$ : gradient-echo, $\mathrm{TR}=$ $500 \mathrm{msec}, \mathrm{TE}=15 \mathrm{msec}, \theta=30^{\circ}$; postcontrast: spinecho, $\mathrm{TR}=600 \mathrm{msec}, \mathrm{TE}$ $=20 \mathrm{msec}$ )

Results: MR imaging demonstrated twice as many erosions in the carpal and metacarpal bones than conventional radiography. In contrast to conventional radiography, MR imaging efforded direct delineation of inflammatory soft-tissue changes. In 4 patients, granulation tissue was demonstrated that infiltrated the sheath of the flexor tendons. Radiocarpal subluxation due to destruction of the radio-scaphoid-lunate ligament was observed in 3 patients. In these patients, MR imaging demonstrated an erosion in the distal radius that was filled with markedly enhancing granulation tissue which extended $1-3 \mathrm{~cm}$ from the articular surface into the marrow. The erosive granulation tissue was thought to have caused the ligamentous destruction initially.

Conclusions: MR imaging is unique in assessing inflammatory soft-tissue changes related to RA and may be used to recognize and prevent potential complications of RA such as tendon rupture or radiocarpal subluxation.

\section{SPACE 1609}

CT and MRI appearances of osteopoikllosis

C. Le Breton (Presenter), P.Saiag, M.F. Carette, E. Baraloux, J.M. Faintuch, G. Delzant, J. M. Bigot; Paris

AIM: To examine the appearance of osteopoikilosis with CT and MRI. Methods: Study of a case of osteopoikilosis discovered fortuitously in a 45 year old man.

Results: Radiological findings consisted of round and oval spots of increased density situated in the metaphyseal and epiphyseal areas of the shoulders and pelvis. The CT showed round and oval dense and homogeneous lesions grouped particularly in the femoral neck along lines of stress. Moreover lesions in the pelvis not seen on the X-ray were identified. The MRI showed the lesions as oval areas of low signal intensity on $\mathrm{Tl}$ and $\mathrm{T} 2$ weighted images.

Conclusions: The distribution of the lesions, the associated skin lesions, the radiographic appearances and those of the CT and MRI typical of multiple bone islands ruled out the diagnosis of metastasis and osseous dysplasias.

\section{SPACE 1610}

\section{Stress fractures of the sacrum}

C. Le Breton (Presenter), K. Tijani, C. Wallays, J.M. Faintuch, P. Rolloy, J. M. Bigot; Paris

AIM: To examine the appearance of stress fractures of the sacrum by CT scan. Methods: 7 cases of stress fractures reviewed retrospectively.

Results: Two appearances were seen: interruption of the cortical bone of the lateral parts of the sacrum with discrete overriding of fragments, and linear areas of sclerosis either with or without clear images inside corresponding to the fracture. The diagnostic in 3 cases had been suspected clinically and on bone scan but never visualised on plain films.

Conclusions: CT scan is the sole method to date capable of showing sacral fractures. This ability is however dependant on technique, requiring a detailed examination centred on the sacrum.

\section{SPACE 1611}

Villonodular synovitis: the use of MRI scanning in 3 cases

C. Le Breton (Presenter), O. Quiroz, F. Capeau, N. Szejnman, E. de Thomasson, L. Richard, M. Schlinger, T. Judet, J. M. Bigot; Paris

AIM: To determine from 3 observations and 26 reported cases in the literature if there exists an appearance on MRI specific to villonodular synovitis (SVN). Methods: The knee was involved in 2 cases and the elbow in one. T1 and T2 weighted images were obtained at 1,5 Tesla with intravenous Gadolinium GTPA in I case.

Results: The diagnosis was indicated in the 3 cases because of intra- and extraarticular soft-tissue masses containing low signal intensity masses at $\mathrm{T} 1$ and $\mathrm{T} 2$ weighted images. The subchondral lytic lesions showed a low signal intensity $\mathrm{T} 1$ and high signal intensity $\mathrm{T} 2$. The diagnosis was confirmed histologically in all cases. Surgery consisted of synovectomy in 2 cases and knee arthroplasty in 1 case. The low signal images in the sequences were explained by the presence of haemosiderin.

Conclusions: $\mathrm{MRI}$ is a useful and atraumatic technique both for diagnosis and management of these lesions.

\section{SPACE 1612}

Cystic chondroblastoma of femoral head: two cases Hervé Leclet (Presenter), Martine Lecomte-Houcke, Pierre-Marie Delforge; Berck-Sur-Mer

Epiphyseal chondroblastoma is a rare benign tumor affecting young people. In 17 to $25 \%$ of cases it is associated to aneurysmal bone cyst (ABC), and is called "cystic chondroblastoma". The cystic aneurysmatic component modifies the imaging aspects. Intra-tumoral mottled calcifications are absent, but is not a specific finding because these calcifications are inconstant. CT scans and T2 weighted MRI images can evoke a cystic chondroblastoma when fluid-fluid levels are identified. The fluid levels are due to cystic component and setting of red blood cells within the cavities. They have been reported to occur in ABC, but are not a specific finding. They can also be observed in other lesions even in giant cells tumor. On pathologic examination, the septa are lined by chondroblastic cells and some multinucleated giant cells. Cystic spaces can involve all the tumor sites and the diagnosis of chondroblastoma can be very difficult. S-100 protein immunostaining is helpful. Chondroblasts and mature cartilaginous cells are stained with S-100 protein, but not giant cells. We report 2 cases of femoral head cystic chondroblastoma. The constellation of 1 . a young people, 2. an epiphyseal lytic tumor and 3 . fluid levels suggests the diagnosis.

\section{SPACE 1613}

\section{Congenital hand macrodactylies: radiologic findings}

R. Mandrioli (Presenter), P. Larini, A. Miselli, U. Ugolotti, P. Prampolini, C. Tremolada, G. Abbiati, F. Draghi; Parma

We discuss the classification and the radiological features of the congenital macrodactylies of the hand. The PURE MACRODACTYLY (or macrodystrofia lipomatosa) is an hyperplasia of all structural elements (tendons, bones, vessels) and mainly of fatty tissue of one or more digits. This malformation is only idiopathic form. The congenital macrodactylies of known etiology can be divided into three groups.

1. Changes due to disorganizied development of cartilage and fibrous tissue:

a) PLEXIFORM NEUROFIBROMA (in von-Recklinghausen disease)

b) ENCHONDROMATOSIS (OLLIER'S disease)

c) MAFFUCCIS SYNDROME (enchondromatosis with subcutaneous hemangiomas)

2. Angiodysplasias, vascular abnormalities developed during fetal growth:

a) HEMANGIOMAS

b) ARTERIO-VENOUS MALFORMATIONS

c) LYMPHANGIOMAS

3. Hypertrophy of skeletal and soft tissues due to haemodynamic factors: 
a) KLIPPEL, TRENAUNAY, PARKE-WEBER SYNDROME (local gigantism, angiodysplasia and cutaneous vascular naevus).

\section{SPACE 1614}

\section{Radiological findings in scoliosis due to lower extremity dysmetria} Augusto Manganiello (Presenter), Francesco Pierazzoli, Andrea Sampieri; Padova

Following 8.000 radiological investigations of the spine and the pelvis carried out in standing position, the authors report that even slight lower extremity dysmetriae can cause different lateral deviations each one with its own, mainly asymmetrical, weight distribution at the lumbosacral level.

Asymmetrical weight distribution plays a very important role in the development of scoliosis because, acting on relatively fixed anatomical structures (discs, sacrum and developing vertebrae), causes asymmetrical narrowing of the disc space between L4-L5 and L5-SI, asymmetrical lowering of the sacrum and axial asymmetry of L4 and L5. Such alterations, modifying the orientation of the resting surface of the sacrum and basal vertebrae, may cause spine deviations on the frontal plane.

If it has been established between scoliotic deviation due to dysmetria and the lateral deviation due to asymmetrical weight distribution an acting synergism or a functional compensation, there will be respectively a progress or a reduction of the curve.

Lasting the dysmetria, curves, initially mobile (paramorphism), may increase and later be fixed (dysmorphism).

\section{SPACE 1615}

\section{C.T. guided needle biopsy of bone}

A. Muñoz (Presenter), M. D. Martorano, E. Añorbe, J. L. Raya, F. Lozano, A. Arenas: Madrid

Percutaneous needle biopsy of bone has become an alternative to the open biopsy for the diagnosis of bone lesions. It is a safe and quick procedure with satisfactory results.

The mayor indication for this technique is suspected metastatic disease. Percutaneous needle biopsy can also be performed in almost any bone lesion (inflammatory or infectious disease, tumors or pseudotumors).

We use two kinds of needles: aspiration type needles (18 to 22-gauge) which are more useful in biopsy of soft tissue areas of the lesion and in lytic lesions. The second type are trephine needles (12 to 17-gauge) which can cut the bone itself. To the date, we have performed 37 C.T. guided percutaneous needle biopsies (metastatic disease (16), osteomyelitis, arthritis, hydatid cyst disease, chondrosarcoma, giant cell tumor, myeloma, histiocytosis $\mathrm{X}$, Ewing sarcoma and lymphoma).

In this exhibit we show the different techniques for percutaneous needle biopsy of bone in the different lesions and the results that we have had in our Service.

\section{SPACE 1616}

\section{US: lesions of the musculoskeletal system}

A. Muro de la Fuente (Presenter), M. Prieto Martin, M.Perez Diaz, R. Robledo Diaz, J. Alarcon Rodriguez, T. Hernandez Cabrero; Madrid

Purpose: This work tries to contribute to the consolidation of the recently obtained role of the US in the evaluation of the Musculoskeletal System pathology. With this purpose we describe the sonographic semiology of the inflammatory, traumatologic and tumoral pathology of the Musculoskeletal System.

Methods and Materials: We present selected images performed with real-time scanners using $5 \mathrm{MHz}$ sector, $7^{\prime} 5$ and $10 \mathrm{MHz}$ trapezoidal and $7^{\prime} 5$ linear transducers.

We mainly study pathology of the periarticular structures of the shoulder, knee and ankle. We also include some descriptions of lesions such as tumors, injuries, inflammations and surgical sequels involving the muscles, tendons, subcutaneous tissue and bone which were examined in different anatomical regions.

Results: We think that Sonography demonstrates that it can be a reliable tool for the diagnosis and evolutive evaluation of the Musculoskeletal System pathology. Besides, the advantages of its inocuity and easy availability can make of this technique the first diagnostic examination to consider in certain cases.

\section{SPACE 1617}

\section{Psoriatic arthritis and erosive osteoarthritis of the hands: a comparison} of erosions

Mark D. Murphey (Presenter), Sharon Foley, John M. Bramble; Kansas City, KS

Purpose: Psoriatic arthritis and erosive osteoarthritis can be clinically and radiographically difficult to differentiate. The presence of bare area erosions has been used to exclude erosive osteoarthritis in distinction from psoriatic arthritis. However, previous studies have not controlled observer bias. The purpose of this study was to determine the incidence of bare area and subchondral erosions in the hands of patients with psoriatic arthritis and erosive osteoarthritis with control of observer bias.

Methods and Materials: We performed a double blind retrospective study separately evaluating 690 interphalangeal and metacarpophalangeal joints in 23 patients with psoriatic arthritis or erosive osteoarthritis. Classic definitions of bare area and subchondral erosions were used. An additional category of combined erosions was developed to reduce ambiguity.

Results: This exhibit demonstrates that bare area erosions constitute 12 percent of erosions in erosive osteoarthritis and were identified in all patients. Subchondral erosions were seen in both disorders but were more numerous in erosive osteoarthritis. We conclude that bare area erosions are frequent in erosive osteoarthritis. The ability to distinguish psoriatic arthritis from erosive osteoarthritis lies in the relative predominance of erosion location.

\section{SPACE 1618}

Radiologic assessment of bone grafting

Mark D. Murphey (Presenter), James L. Quale, David J. Sartoris, John M. Bramble; Kansas City, KS

Purpose: Bone is the second most frequently transplanted tissue with more than 200,000 procedures annually in the United States. Bone grafting thus constitutes a vital tool in the management of many orthopedic abnormalities. Radiologic evaluation of bone graft has unfortunately received only limited attention. This exhibit illustrates the radiographic appearances of the various types of bone graft both normal and abnormal.

Methods and materials: This exhibit demonstrates the normal appearance of many types of osseous implants including autograft, allograft, osteochondral graft, and bone substitutes. Abnormal radiographic alterations of bone graft failure from infection, fracture, nonunion and graft rejection are also stressed. The utilization of multiple modalities including conventional radiography and tomography, scintigraphy, CT and MR imaging are illustrated.

Results: Knowledge of the radiographic techniques available and their appropriate application as well as an awareness of the roentgenographic appearance is imperative to evaluate osseous implants. This exhibit illustrates these features with conventional radiography as the foundation upon which diagnostic imaging is based. Indeed, radiographic assessment often provides the initial information of successful bone grafting procedures. Perhaps more importantly, however, early complications may be recognized allowing improved management and ultimate clinical outcome.

\section{SPACE 1619}

Abnormalities of the sacroiliac joint

Mark D. Murphey (Presenter), James L. Quale, Louis H. Wetzel, John M. Bramble, Solomon Batnitzky; Kansas City, KS

Purpose: Numerous pathologic conditions involve the sacroiliac joint and symptoms from these various abnormalities may be indistinguishable from mechanical causes of low back pain. Consequently radiologic assessment plays a major role in evaluation. This exhibit illustrates the radiographic appearances of these disorders in and about the sacroiliac joint.

Methods and Materials: This exhibit will present the wide spectrum of pathology that involves the sacroiliac joint including the spondyloarthropathies, other arthritides, pyogenic arthritis, trauma, and secondary neoplastic extension. We will demonstrate and contrast the utilization of multiple modalities (conventional radiography and tomography, CT, MR imaging, and scintigraphy) in investigation of this interesting articulation.

Results: Conventional radiography remains the primary screening modality for disease about the sacroiliac joint. Unfortunately the complex anatomy of the sacroiliac joint frequently makes interpretation of conventional radiographs difficult and findings are often initially subtle. CT and MR imaging are however, becoming increasingly important because of their high sensitivity enabling earlier detection of disease and their ability to accurately evaluate soft tissues in and around the sacroiliac joint.

\section{SPACE 1620}

\section{Phosphor plates in musculoskeletal radiology}

Mark D. Murphey (Presenter), James L. Quale, Samuel J. Dwyer III, Arch W. Templeton, Larry T. Cook, John M. Bramble, Norman L. Martin; Kansas City, KS

Purpose: Computed Radiography (CR) is a relatively new digital radiographic technique employing a photostimulable phosphor as the $\mathrm{x}$-ray detector and is an evolution of conventional analogue radiography. This exhibit demonstrates our experience utilizing a $2 \mathrm{~K} \times 2 \mathrm{~K} \times 10$ bit phosphor plate $\mathrm{CR}$ system for musculoskeletal examinations.

Methods and Materials: This exhibit illustrates a wide spectrum of musculoskeletal applications (portables, spine series, scoliosis, pelvis, and extremities) 
and pathology using CR. Use of an interactive workstation and advantages and disadvantages of computed radiography technology are also demonstrated. An observer performance investigation comparing conventional radiographs to phosphor plate images was performed on 100 skeletal examinations with various levels of dose reduction.

Results: The observer performance study suggests that the spatial and contrast resolution of our phosphor plate system is adequate for the vast majority of clinical applications in musculoskeletal imaging. Exposure dose reduction of $30-50 \%$ is possible without loss of diagnostic accuracy and the interactive workstation provides an effective method to modify display parameters and salvage improperly obtained images. We conclude that the $2 \mathrm{~K} \times 2 \mathrm{~K} \times 10$ bit $\mathrm{CR}$ phosphor plate system is an alternative to conventional film-screen images in evaluation of the musculoskeletal system without significant differences in diagnostic quality.

\section{SPACE 1621}

Muscular sarcoidosis: radiologic dlagnosis

Shoichiro Otake (Presenter), Hirokazu Mizutani, Satoru Ohba, Masaharu Noda, Masahiko Yamamoto; Nagoya

Purpose: Muscular sarcoidosis is a granulomatous disease and often forms intramuscular mass lesions which should be differentiated from neoplastic tumors. However imaging analysis has not been described enough. We present radiological appearances of this rare disease.

Methods and Materials: Fifteen patients with histopathologically proved muscular sarcoidosis were examined. MR was performed in all the patients, CT was in 5 patients, gallium imaging study was in 10 patients and angiography was in 1 patient.

Results: In all the patients, T2-weighted axial images demonstrated star-shaped decreased intensity lesion within increased intensity mass lesion. T2-weighted coronal and sagittal images clearly delineated longitudinally extension of the mass with decreased intensity area sandwiched between increased intensity areas. Correlation with histology revealed central decreased intensity area was dense fibrous tissue and peripheral increased intensity area was inflammatory granulomas. Gallium imaging study demonstrated increased uptake along longitudinally extended lesion, however, the detectability was lower than that of MR. CT showed poor tissue-contrast between mass and normal tissues. Angiography performed for a large sarcoid nodule showed non-specific hypervascular pattern. The results suggest $M R$ is a most useful imaging tool for detecting and characterizing muscular sarcoidosis.

\section{SPACE 1622}

\section{Ultrasonography of the shoulder, a teaching approach}

J. Piqueras Pardellans (Presenter), R.Domínguez Oronoz, I. Castells Ferrer, A. Grifé Balasch, D. Casas Curtó; Barcelona

Ultrasonography is an useful technique for study of periarticular diseases of the shoulder joint. It has some limitations, but is an easy way to perform an initial study. This technique is not very well known even within the radiology specialists.

We have edited a videotape intended to teach a systematic study of the shoulder joint by ultrasound, with normal anatomy, ultrasound and pathology correlation.

In a step by step method the technique and the most usual pathologies are depicted. (Fluid collections, calcifications, tendinitis, tendon rupture, rotator cuff tears).

The video includes cadaver dissection, animation schemas, and real-time video from normal volunteers and patients.

The studies have been performed with 2 linear $(5 \mathrm{MHz})$ transducers, with and without ultrasound pads.

\section{SPACE 1623}

Osteoarticular radiological findings in hemophilia J. Pomés (Presenter), E. Salvadó, G. Santamaría, P. Riba, J. Monteagudo, M.R. Bueno, M. Velasco; Barcelona

Purpose: To review the radiological features of osteoarticular complications in hemophilia.

Methods and Materials: A total of 20 hemophiliac males and 1 female with Von Willebrand disease, aged between 12 to 70 years (mean 30), were followed in our hospital for a ten years period. All patients had osteoarticular complications, with a total of 60 joints affected. 15 had severe factor deficiency, and 6 had moderate deficiency. At initial presentation, 2 patients with moderate factor deficiency had a pseudotumor. One was intraosseous and developed without previous injury. All patients were studied with conventional radiography and those with pseudotumor were referred for scintigraphy, computed tomography (CT) and magnetic resonance (MR) imaging studies.

Results: The affected joints were: knee (17), ankle (16), elbow (16), hip (2), others $(9)$. The main radiological findings were: erosions of joint margins $(50)$, narrowing of joint space (48), sclerosis and osteophytoses (39), enlarged epiphysis (33), osteoporosis (22), and intraarticular effusion (11). Regarding patients with pseudotumor, the involved bone were: the radius and the femur. CT was able to detect blood density within the pseudotumor (HU: 77 ) and with MR many different combinations of signal intensities on T1-and T2-weighted images were observed.

\section{SPACE 1624}

Therapeutic embolisation of aneurysmal bone cysts and bone tumors B. Radanović (Presenter), S. Šimunić, Ż. Vitezica, I. Stanić, D. Orlić, J. Stojanović, B. Oberman; Zagreb

The report describes results of therapeutic embolization of aneurysmal bone cysts and bone tumors in 16 patients. Therapeutic embolization was undertaken in 10 patients with aneurysmal bone cyst, 1 patient with giant cell tumor, 3 patients with intraosseous hemangioma and 2 patients with solitary metastasis from thyroid gland follicular carcinoma.

Angiography to evaluate tumor process was performed prior to embolization, while embolization was done during the same session or later.

Therapeutic embolization was performed with Ivalon, Marbagelan particles and Gianturco coils. The postembolization period was characterised by complete or marked pain relief in all cases. Also, all patients experienced subjective improvement as well as decrease in size of tumors. Patients whose follow-up was longer than 12 months had objective evidence of sclerosis and bone recalcification.

There were no complications during therapeutic embolization procedure.

\section{SPACE 1625}

Evaluation of acetabular cup orientation in computed tomography and radiography: phantom and clinical studies

Dorothee Radtke (Presenter), Roman Sokiranski, Stefan Exner, Michael Seidel, Andreas Radtke, Rahim Rahmanzadeh; Berlin

Several radiographic methods for the evaluation of socket angles after total hip replacement have been proposed, but a precise and reproducible method does not exist. We want to show that computed tomography offers an easy and reliable method for determining acetabular cup position.

A phantom was constructed that allows positioning a metal ring corresponding to the rim wire of the socket in various combinations of lateral opening and version. On CT images this ring is represented by two points, which are well characterized by the coordinates $x, y$ and $z$. After determination of those coordinates angles can be calculated. Conventional radiographs and CT images were reviewed in 50 patients, who had been examined because of suspicion of prosthesis loosening, with regard to the possibility of determining acetabular cup orientation.

There was good correlation between the true and calculated angles in computed tomography and we could easily distinguish between ante- and retroversion, which is virtually impossible on a single radiograph.

A method of metal artifact reduction makes possible routine employment of computed tomography even in diagnosis of metallic implants. Used for evaluation of total hip replacement computed tomography is offering an easy and highly accurate method of determining acetabular cup position.

\section{SPACE 1626}

Acetabular fractures: value of three-dimensional CT imaging J.J. Railhac (Presenter), M. Brunet, P. Maquin, P.Samaran, J. Assoun, E. Bloom; Toulouse

Purpose: Evaluation of $3 \mathrm{D}$ imaging in the pre-operative analysis of acetabular fractures.

Methods: Twenty-five patients with a recent acetabular fracture were submitted to plain radiographs and conventional CT scans along with 3D imaging. All patients were evaluated for the type of the fracture, degree of comminution and displacement, intra-articular fragments. Each of the techniques was compared in respect to its diagnostic accuracy.

Results: - 3D imaging did not bring any complemental information than conventional explorations. Plain radiographs and axial CT scans were more sensitive than the $3 \mathrm{D}$ images in detecting undisplaced fractures.

- However, $3 \mathrm{D}$ technique allows a perfect spatial representation of the lesion. The multidirectional view is most helpful in the evaluation of the importance, the disruption and the orientation of the bone fragments. It has an overwhelming interest in the study of the fractures involving posterior acetabular wall and comminuted fractures which analysis is known to be most difficult. It provides the surgeon with an anatomical representation of the lesion, much alike its surgical appearance. 


\section{SPACE 1627}

The compressive strength of trabecular bone in patients with different kinds of osteopenia by means of a mechanical test

W. S. Rau (Presenter), B. Nägele-Wöhrle, A. Schulz, K. Rauber, H. Stracke; Gießen

Radiological measurements of trabecular bone mineral density show only a moderate correlation to the risk of vertebral fracture and are not able to distinguish between osteoporosis, osteomalacia and hyperparathyreoidismus. In borderline cases, a mechanical test of compressive strength should be useful. It is our aim to develop such a test, that is applicable in vivo.

In patients with blurred osteopenia we take fluoroscopically guided Jamshidi needle biopsies of the iliac crest as a matter of routine. The diameter of the bone core is $2 \mathrm{~mm}$, the length ranges from 30 to $65 \mathrm{~mm}$. The larger part of the bone core is investigated microscopically, while an exactly $10 \mathrm{~mm}$ long section is compressed in an especially developed apparatus by twisting a micrometer screw. During compression, the exerted force and the resulting loss of height were measured and registered continuously.

To check the validity of these experiments, we removed two or more complete vertebral bodies of cadavers and measured their mineral content by quantitative computed tomography.

Load-deformation experiments were carried out on one vertebral body in toto, on several biopsies from the adjacent vertebral body and on several biopsies from the iliac crests of each cadaver.

Load-deformation experiments and quantitative computed tomography show surprisingly non-uniform results. Histomorphometric analysis of trabecular bone architecture seems to be necessary.

\section{SPACE $\underline{1628}$}

\section{Subacromial pathology: diagnostic imaging}

Luciano Riboldi (Presenter), Luca Belli, Carlo Neri, Maurizio Mentasti, Cristiana Vanoli; Varese

Painful shoulder and impingement syndrome with or without rotator cuff tears can be evaluated by different procedures: plain radiography, arthrography, ultrasonography, CT arthrography and MRI.

Our purpose is to compare the imaging of the different methods.

For each technique we selected 15 patients affected by subacromial disease in order to delineate advantages, disadvantages and usefulness of each imaging technique.

The results were correlated with surgical findings or clinical follow-up. On the basis of this study, our opinion is that each imaging technique we tested keeps its own indications in the evaluation of the subacromial disorders and no one of them is of absolute value.

\section{SPACE 1629}

Radiological approach to variable spine plating systems (V.S.P.) with oblique views and C.T. scans

A. Rivas (Presenter), E. Caceres, R. Perez-Andres, A. Grife-Balasch; Badalona

Purpose: To describe a new radiologic method to evaluate the success and failure of pedicle and vertebral body screw placement due to unreliability of radiographic studies utilized since now in these spinal instrumentations.

Methods and Materials: 39 patients underwent a V.S.P. from October 1988 to August 1990. 31 patients (25 Steffee and 6 Luque devices) were included in the study by means of the following sequence: plain AP and lateral films, 60 degrees oblique projections and C.T. scan series through the pedicles and vertebral bodies. Correct pedicular and vertebral cortex images were obtained in all patients, permitting the radiographic evaluation of success and failure in these techniques. C.T. metal artifact was diminished performing oblique transpedicular scans.

Results: 113 pedicles and 57 vertebral bodies were evaluated obtaining the following results:

A. - Radiologic failure detected by C.T.

- pedicle cortical penetration (lateral or medial) ....... 23/113 $(20.3 \%)$

- vertebral body cortex penetration ................. 37/113(32.7\%)

- unperforated anterior sacral cortex............... 16/37 (43.2\%)

B. - Radiologic failure detected by 60 degrees oblique views

- pedicle cortical penetration (upper and lower) ......... 2/112 (1.8\%)

C. - Postoperative complications $\ldots \ldots \ldots \ldots \ldots \ldots \ldots \ldots \ldots \ldots \ldots \ldots \ldots+31 \quad(9.6 \%)$

- infection (1)

- broken screw (1)

- root injury related to the screw penetration through the inner pedicular cortex (1).

\section{SPACE 1630}

Pustulotic arthro-osteitis and related conditions: CT findings A. Rivas (Presenter), R. J. Perea, M. Busto, A. Olive, J. D. Casas, X. Tena; Badalona

Purpose: To describe four new cases of pustulotic arthro-osteitis seen in our hospital between 1984-1990, emphasizing CT findings.

Methods and Materials: All patients fulfilled palmoplantar pustulosis and/or swelling and tenderness on anterior chest wall with or without lumbosacral pain. Other conditions as traumatic, tumoral, infectious and HLA-B27 diseases were ex cluded by means of a clinical questionnaire, a radiological study and laboratory tests. All patients were examined by CT scan performing contiguous scans (slice thickness $2.5 \mathrm{~mm}$, slice increment $2-2.5 \mathrm{~mm}$ ) with angulation of the gantry to a position perpendicular to the manubrium. Multiplanar CT images were obtained.

Results: 2 patients $(50 \%)$ were female and $2(50 \%)$ male, mean age 42.2 (range 32-53). All patients were having anterior chest pain, half of them palmoplantar pustulosis and $3(75 \%)$ lower back pain. B27 was negative in all patients and pathological studies showed chronic inespecifical inflammation.

Hyperostosis, sclerosis and erosions over manubrium and first chondroesternal joints were the CT hallmarks in all patients. Furthermore, ilial or sacral sclerosis always unilateral without narrowing of the joint space was seen in 3 $(75 \%)$ patients. A sclerotic (ivory) vertebra was seen in one ( $25 \%)$ patient.

\section{SPACE 1631}

Normal and pathological MRI features of the posterolateral angle point (PLAP)

B. Roger (Presenter), J.Y. Lazennec, C. Grataloup, D. Folinais, M. LavalJeantet; Paris

a) Aim of the study: The authors present a study of the normal anatomy of the posterolateral angle point in a cadavre, the normal MRI features and the various lesions observed, ranging from simple confusion of the popliteal tendon to complete rupture of the PLAP.

b) Methods: The use of T1 MRI sequences in conjunction with intravenous injection of a paramagnetic agent $\left(\right.$ Dotarem $\left.^{\oplus}\right)$ is extremely helpful for better semiologic analysis of injury.

c) Results: They recall that the posterolateral angle point consists of the popliteal muscle and the following capsulo-ligamentous elements: the arcuate popliteal ligament, the fibular collateral ligament, the lateral condylar capsule and the posterior cornu of the lateral meniscus. This anatomical description would appear to be essential as it has now been clearly established that chronic anterior laxity of the knee is due to destabilisation of the lateral condyle secondary to loss of the two principal stabilising elements of the lateral compartment: the anterior cruciate ligament and the popliteus within the posterolateral angle point.

d) Conclusions: At present, MRI is the only non-invasive method which provides evidence, during the same examination, of both central pivot injury and peripheral damage, especially of the popliteal area, with great diagnostic accuracy.

\section{SPACE 1632}

MRI features of traumatic lesions of the posterior cruciate ligament (P.C.L.)

B. Roger (Presenter), C. Grataloup, D. Folinais, P. Christel, M. LavalJeantet; Paris

a) Aim of the study: Lesions of the posterior cruciate ligament are less frequent than lesions of the anterior cruciate ligament and often pass unnoticed because of their relatively good tolerance and the absence of any early formal signs allowing establishment of the diagnosis. Therapeutic approaches also appear to be less well defined with a current tendency towards orthopaedic treatment by plaster immobilisation in slight flexion. The efficacy of treatment depends on how early it is instituted.

b) Methods: For this study, the files of 25 patients who underwent an MRI examination for PCL damage were reviewed. Mutual comparison of MRI findings with the clinical, arthroscopic and/or arthrotomic examinations was made.

c) Results: By directly demonstrating the posterior cruciate ligament, with an extremely sensitive analysis of the lesions, MRI is useful in minor forms in which the posterior laxity is insufficient to conclude on the diagnosis or in complex forms associated with rupture of the central pivot. In recent lesions, the rupture is generally situated in the upper part of the ligament and is reflected by a high intensity signal on T2-weighted sequences. In the forms seen some time after the trauma, it is mainly the morphology of the ligament which is modified: distended, sinuous, of variable thickness with localised cicatricial intraligamentous signal changes. Injection of paramagnetic contrast agent $\left(\right.$ Dotarem $\left.{ }^{\oplus}\right)$ provides more accurate analysis of injured ligaments.

d) Conclusions: MRI of injured knees should therefore allow an earlier and faster diagnosis of lesions of the posterior cruciate ligament. Although the most 
frequent current therapeutic approach consists of palliative orthopaedic treatment, a better diagnostic approach should allow an improvement in the treatment.

\section{SPACE 1633}

MRI signs of non-ligamentous pathologies of the intercondylar notch B. Roger (Presenter), C. Grataloup, D. Folinais, M. Laval-Jeantet; Paris

a) Aim of the study: The extrasynovial and intracapsular intercondylar notch is inaccessible to arthrographic investigations when the synovium remains intact. Under normal conditions, it essentially contains the cruciate ligaments and fatty connective tissue.

b) Methods: Direct visualisation of this region by MRI enabled the authors to identify a disease poorly demonstrated by other investigation techniques in 14 patients. There is a modification of the signal of the intercondylar notch with no abnormalities of the cruciate ligament.

c) Results: The lesion shows a low-intesity signal on $\mathrm{Tl}$-weighted sequences (less marked than that of the ligaments) and a very marked high-intensity signal on T2-weighted sequences. It is associated with loss of the fatty connective tissue and a convex appearance of the capsule due to the mass effect. The absence of intraarticular effusion confirms the extrasynovial site of the lesion. In every case, the patient presents clinically with a posterolateral pain syndrome with discomfort during knee flexion. Both testing and the arthrographic assessment are normal. Two etiologies are detected: pseudocystic liquid lesion and a mixed, cystic and angiomatous degenerative lesion. Injection of paramagnetic contrast agent (Gd-DOTA, i.e. Dotarem ${ }^{\circledast}$, Guerbet) allows differentiation between the two aetiologies. The signal of the pure cystic form does not vary between $\mathrm{Tl}$ - and contrast-enhanced $\mathrm{Tl}$-weighted sequences, while the cystic and angiomatous form is contrast enhanced. d) Conclusions: MRI is therefore valuable for the diagnosis of these lesions and for proposing simple treatment, particularly in the cystic form with puncture-evacuation under ultrasound guidance.

\section{SPACE 1634}

Rhabdomyolysis in computed tomography: a case report

K. Rosenkranz (Presenter), C. Zwicker, R. Langer, M. Langer, M. Wilfling, R. Felix; Berlin

Purpose: In a patient with arterial occlusive disease, dynamic CT was performed in order to exclude abdominal abscess after an aorto-bifemoral bypass operation.

Patients and methods: A 59-year-old patient developed after an aortobifemoral bypass operation an acute renal failure due to rhabdomyolysis requiring hemodialysis. In addition to a myoglobinuria and increased levels of serum urea nitrogen and creatinine, serum creatine kinase was shown to be elevated immediately after the operation up to $81600 \mathrm{U} / \mathrm{l}$. Because of continuous fever, dynamic CT (Somatom Plus, Siemens, $100 \mathrm{ml}$ Ultravist 370) was performed to exclude abdominal abscess.

Results: In CT, no abdominal abscess was demonstrated. In contrast to preoperative $C T$, the muscles of the back and the gluteal region showed an irregular inhomogenous contrast enhancement as a correlate of rhabdomyolysis.

This case was compared to other data already published.

\section{SPACE 1635}

So-called femoral head anteposition after operative treatment of CDH and its reasons in computerised tomography

K. Rzymski (Presenter), M. Napiontek; Poznań

The clinical appearance of so called femoral head anteposition recognised as a rare complication after operative treatment of $\mathrm{CDH}$ has been described. This clinical picture comprises a bulge in the groin corresponding with femoral head, fixed external rotation of the extremity and movement restriction in the operated hip. Computerised tomography scans in the horizontal and sagittal plaines of 22 "antepositioned" hips from 22 children operated on because of $\mathrm{CDH}$ were studied. Among those, 15 hips from children aged between 2 y $2 \mathrm{~m}$ and 8 y $3 \mathrm{~m}$ were primarily operated at our Clinic by open reduction with subtrochanteric varus derotation osteotomy with shortening of the femur and transiliac osteotomy described by Dega. There were 7 hips from children aged between 1 y and 2 y $6 \mathrm{~m}$ operated primarily at other hospitals where similar operative treatment was attempted. An evaluation of CT scans and clinical examination combined, made possible to differentiate homogeneous clinical appearance of so called femoral head anteposition and establish its one or several coexisting reasons: anterior dislocation of the hip, coxa magna after avascular necrosis, gluteal muscles fibrosis and periarticular ossifications.

\section{SPACE 1636}

\section{Uitrasonography of the shoulder}

F. Sáez (Presenter), J. C. Martin, J. Negueruela, E. Elizagaray, J. Calonge, I. Azkuna; Bilbao

Purpose: To evaluate and show the possibilities of ultrasound in the diagnosis of shoulder pathology.

Methods and Materials: 187 patients have been studied by means of ultrasound, with arthrography and CT-arthrography as gold standards. Bilateral ultrasound was performed with two $7.5 \mathrm{MHz}$ sector and linear array transducers. Static and dynamic projections have been practised.

Results: We present illustrative cases of the different lesions that can be imaged with ultrasound:

Rotator cuff: tendinitis (enlargement of the tendon); partial tear (flatening of the normal superior convexity of the cuff, focal thinning): full-thickness tear (superior concavity, focal defect, non-visualization of the cuff)

Subacromial-subdeltoid bursa: bursitis (fluid, easier to see in the impingement position).

Glenohumeral fluid: increased distance from capsule to humerus (axillary projection) and from capsule to glenoid (posterior projection).

Humeral head contour: irregularity, occult fractures, Hill-Sachs lesion.

Muscle atrophy: changes in echogenicity and thickness of the supraspinatus (superior approach), infraspinatus (posterior approach) and deltoid muscles. Conclusions: Ultrasound is an important and cost-effective tool for diagnosis of shoulder pathology.

\section{SPACE 1637}

\section{Painful/unstable shoulder, CT-arthrography}

F. Sáez (Presenter), E. Elizagaray, J. Negueruela, J. C. Martin, J. M. Peña, M. J. Ereño; Bilbao

Purpose: To show the diagnostic performance of CT-arthrography of the painful/unstable shoulder.

Methods and Materials: 187 patients with painful or unstable shoulder have been studied by means of CT-arthrography.

Results: We have encountered a great variety of painful shoulder pathology, including impingement syndrome with different degrees of involvement (ranging from tendinitis to partial and complete rotator cuff tears), cuff tear arthropathy, lesions of the long head of the biceps tendon (tendinitis, rupture, subluxation and luxation), calcifying tendinitis, adhesive capsulitis, dead arm syndrome and degenerative joint disease.

Lesions related to instability include cases of capsular avulsions (both from the scapular and humeral necks); disruption of the glenohumeral ligaments; anterior (fibrous Bankart lesion), inferior and posterior labral pathology; anterior (osseous Bankart lesion) and posterior glenoid fractures; posterolateral notch in the humeral head (Hill-Sachs lesion) and changes in glenoid angulation.

Miscellaneous conditions: post-radiation changes, hemophilia, Paget disease, tumors.

Conclusions: CT-arthrography is a reliable exploration for both instability and painful problems of the shoulder.

\section{SPACE 1638}

\section{Rrr.'ımatoid knee: Gd-enhancement MR imaging}

Keiko Sakuyama (Presenter), Yukio Oyama, Osamu Miyazaki, Yasuo Nakajima, Mitsuaki Saeki, Yasuyuki Seino, Kazuhito Haji, Hiroshi Mitsuhashi; Jokohama

Purpose: To determine whether it was possible to distinguish between synovial fluid and inflammatory pannus by MRI with contrast enhancement, we evaluated patients with known rheumatoid arthritis.

Methods and Materials: Fourteen patients (14 joints) with known rheumatoid arthritis were imaged with a $1.5 \mathrm{~T}$ MR system with surface coil. The scans were obtained in the coronal and sagittal planes with T1-weighted and T2-weighted images. Gd-enhanced $\mathrm{Tl}$-weighted images were also obtained.

Results: Ten patients with symptom of pain and/or swelling of the knee showed collection of low signal intensity about the knee joint on precontrast T1-weighted images, and high signal intensity in the same area on T2-weighted images. Only Gd-enhanced $\mathrm{Tl}$-weighted images could make a differentiation between the pannus formation and the fluid as inhomogeneous enhancement and without enhancement respectively. 


\section{SPACE 1639}

Chronic osteomyelitis (OM): comparison of magnetic resonance imaging (MRI) and white blood cell scintigraphy (WBC)

J.Sciuk (Presenter), G. Bongartz, B. Greitemann, K. Scheidhauer, O. Schober, P.E. Peters; Münster

This prospective study compares the accuracy of MRI and WBC in the evaluation of chronic OM.

33 patients with suspected chronic OM, 18-75 years of age $(14 \mathrm{f}, 19 \mathrm{~m})$ were examined. MRI $(1,5 \mathrm{~T})$ was performed with $\mathrm{T} 1$-weighted $(\mathrm{TR}=600 \mathrm{~ms}$, $\mathrm{TE}=15 \mathrm{~ms})$ and $\mathrm{T} 2$-weighted $(\mathrm{TR}=2500 \mathrm{~ms}, \mathrm{TE}=90 \mathrm{~ms})$ Spin-Echo $(\mathrm{SE})$ sequences, a T1-weighted FLASH $90^{\circ}$-kinetic during rapid injection of Gd-DTPA $(0,1 \mathrm{mmol} / \mathrm{kg})$ and a Gd-enhanced Tl-weighted SE-sequence. For WBC about $500 \mathrm{MBq}$ Tc-99m-labelled monoclonal mouse antibodies were injected. Imaging was performed after 4 and $24 \mathrm{hrs}$.

Of 24 cases, proved to be positive by open surgery and histology, 21 were correctly diagnosed by MRI and 19 by WBC. Every OM could be detected with at least one of the both imaging methods. All false negative cases of WBC were located in the spine, those of MRI in the extremities . 8/9 non-infectious lesions were true negative with both modalities. (Sensitivity MRI: $88 \%$, WBC $79 \%$. Specificity: MRI $89 \%$, WBC $89 \%$. Accuracy: MRI $88 \%$, WBC $82 \%$ ). MRI gives more detailed information regarding the extent of the involvement and is superior in OM of the axis. WBC is more sensitive in peripheral OM, has advantages in polytopic $O M$ and in metallic implants.

\section{SPACE 1640}

Infection versus neoplasm in the spine, differentiation with MR imaging H.S. Sharif (Presenter), M. C. Haddad, O.A. Aideyan, D. C. Clark, M. Shahed, A. Qureish, M. Y. Aabed, T. Baydounn, J. Ross; Riyadh

Purpose: To assess the value of MR imaging for differentiating infection from neoplasm and for distinguishing the different forms of spine infection.

Methods and Materials: Four radiologists performed blinded interpretation of spinal MR examinations of 149 patients with proved infection or neoplasm before and after applying proposed MR criteria for the different forms of spine infections and neoplasms. Criteria for the infections $(n=89)$ included involvement of two or more consecutive vertebrae and disks. Specific criteria for tuberculosis included skin lesions, gibbus deformity, posterior element involvement, paraspinal abscesses, and focal intraosseous abscesses demonstrated only with Gd-DTPA. Criteria for metastases $(n=42)$ included multiple vertebral involvement with moderate soft-tissue component, while primary neoplasms $(n=18)$ involved part or whole of a vertebrae, with posterior elements being affected in both.

Results: Accuracy for differentiating infection from neoplasm ranged from $84 \%-91 \%$ without use of the criteria and from $87 \%-95 \%$ with the criteria. Accuracy for distinguishing the different forms of infections ranged from $70 \%-91 \%$ without and $84 \%-91 \%$ with the criteria. Diagnostic pitfalls included neoplasms involving disks, infections and neoplasia invading the spine by direct extension, and fungal infections.

Conclusions: MR imaging is an accurate method for differentiating infection from neoplasm in the spine and also for differentiating between the different forms of spine infections.

\section{SPACE 1641}

Transverse geniculate ligament of the knee: appearance on plain films S. A. Sintzoff jr. (Presenter), P.A. Gevenois, C. Matos, I. Gillard, Y. Andrianne, J. Struyven; Bruxelles

Purpose: We wish to report the appearance of a normal anatomic structure, the transverse geniculate ligament of the knee, which we have seen in 4 patients on lateral plain films of the knee. To our knowledge it has only been described on MRI examinations.

Methods and results: Our 4 patients had symptoms attributed to meniscal tears diagnosed by MRI and confirmed by arthroscopy. On lateral plain films of these patients, the transverse geniculate ligament could be seen as a small rounded opacity surrounded by fat density, situated anterior to the knee joint. MRI examinations were available for all these patients and demonstrated a thick, normal intensity transverse ligament. In all cases, this ligament was separated from the capsule by fatty tissue. This feature made the ligament evident on laterial plain films.

Conclusions: The transverse geniculate ligament of the knee is a normal anatomic structure that should be recognised when seen on lateral plain films of the knee.
SPACE 1642

Ultrasound examination of the hand in the diagnosis of rheumatoid arthritis

M. Sperandeo (Presenter), A. Varriale, G. Sperandeo, G. Cozzolino; Napoli

In patients affected by rheumatoid arthritis, tendon and soft tissue involvement of the hands and wrists detected by ultrasonography (US), are not revealed by conventional X-Rays which can delineate bone erosive lesions only; even if digitalized techniques have recently improved diagnostic accuracy of X-Rays. Early diagnosis is established today by the use of US which makes a careful detection of the extent of joint involvement possible. Thirteen patients with rheumatoid arthritis ( 10 women and 3 men), aged 39-66 years, were studied. All subjects were examined by X-Rays and US of the hands and wrists in static and dynamic conditions. Linear array real-time transducers ( 3.5 and $5 \mathrm{MHz}$ ) and a $7.5 \mathrm{MHz}$ sectorial array, using a gel material as a water delay system (Kiteko $3 \mathrm{M}$ ) were used. US resulted to be very accurate in showing the hypoechoic swelling of soft tissue surrounding ulna extremity and the hypoechoic metacarpo-phalangeal joint lesions. Moreover the "fuse-shaped" thickening of the joint capsule because of hypertrophic teno-synovitis was delineated. Finally by a dynamic examination, performed during flexion and extension maneuvers, eventual tendon involvement was evaluated. In conclusion, US may be considered a reliable and non-invasive first approach procedure both in establishing the diagnosis of rheumatoid arthritis and in the follow-up of patients with long-standing disease. It should be performed in addition to X-Rays in order to improve diagnostic accuracy.

\section{SPACE 1643}

Changes in bone mineral density in renal calculosis I. Delov, S. Tsvetkova, S. Tabakov (Presenter); Plovdiv

It is known that renal calculosis and especially the oxalate one is associated with certain changes in calcium homeostasis. Therefore we have studied spinal bone mineral density (BMD) as a referee for bone mineral content (BMC) in patients with nephrolithiasis.

Methods: Single-energy quantitative computed tomography (QCT) has been performed on a CE 10000 scanner using a $\mathrm{K}_{2} \mathrm{HPO}_{4}$ calibration phantom. The second and the third lumbar vertebrae $\left(\mathrm{L}_{2}-\mathrm{L}_{3}\right)$ trabecular $B M D$ has been measured. An empirical fat correction has been made. The study comprises 60 patients males and females, aged 25-65 years.

Results: We established that in all cases BMD had been lower than the age mean values. The percentage bone loss varied from $5-10 \%$ up to $35-40 \%$ and was related with the type of the calculosis as well as with its heaviness and duration. In patients with oxalate calculosis BMD was $27 \%$ in average lower than the statistical age mean values.

Conclusions: The significant bone loss in nephrolithiasis should be taken into account when making an effective therapy.

\section{SPACE 1644}

Radiologic examination of the rheumatoid wrist

M. Taljanović (Presenter), Z. Merhemić, A. Mahić, L. Gluhbegović, A. Buljina; Sarajevo

In 25 patients with rheumatoid arthritis were examinated wrist plain film radiographs, wrist arthrography and digital termovision. We compared the results of the obtained methods, evaluating the presence and the extent of synovial inflammation.

\section{SPACE 1645}

CT and MR diagnosis of intraosseous lipoma of the ankle X. Tomás (Presenter), C. M. Rovira, X. Perich, J.A. Clavero, A. Salvador, J. Peri, A. Rovira; Barcelona

1. We describe the radiologic findings observed in five patients with ankle intraosseous lipoma (IL), a relatively rare benign tumour of bone. We want to demonstrate that a definitive diagnosis can be made with CT and MR.

2. Preoperative radiographs, clinical histories, CT and MR $(0.5 \mathrm{~T})$ findings of five male patients aged from 31 to 62 years were reviewed. Four of them had IL of the calcaneus: the fifth patient had IL of the tallus.

3.8 to $15 \%$ of IL occurs in the calcaneus; we have not found reported cases in tallus. In this tumour a presumptive diagnosis can be made by X-ray films, but we can get a definitive diagnosis with CT by measuring the CT attenuation numbers of the lesion, which fall within the range of fatty tissue. Thus, MR specific relaxation times of the tumour, with a very short $\mathrm{Tl}$, confirm the presumptive diagnosis; all those findings can avoid any surgical biopsy. 


\section{SPACE 1646}

The study of clinical utility of positive imaging in soft tissue tumors with 99mTc(v)-DMSA

Wang Rongfu (Presenter); Suresnes

2-3 dimercaptosuccinic acid (DMSA) is generally taken as a renal cortical imaging agent. This work has reported that DMSA, labelled with $99 \mathrm{mTc}$ at $\mathrm{PH}$ $899 \mathrm{mTc}(\mathrm{v})$-DMSA by modifying method, was administered intravenously to 20 patients ( 15 to $20 \mathrm{mCi}$ ) with and without soft tissue tumors after stationing at 30 minutes. Labelled $99 \mathrm{mTc}(\mathrm{v})$-DMSA was evaluated by thin layer chromotography (TLC), the labelling yield $\geq 96 \%$ and the radiochemical purity of $98 \%$. SPECT was performed $1,2,4$ and $24 \mathrm{hr}$ respectively after injection. I case was not always imaged. We observed important accumulation of radioactivity in primary and secondary lesions in 17 patients with various benign and malignant soft tissue tumors. 2 patients with abdominal occult abscess surgically determined were slightly imaged. Tumors were relatively visualized already after $1 \mathrm{hr}$ but the uptake was more intense at $2 \mathrm{hr}$. The targetto-nontarget ratios at $2 \mathrm{hr}$ varies from 1.8 to 2.5 . Biodistribution studies in mice and human showed that renal uptake was minute. It is concluded that $99 \mathrm{mTc}(\mathrm{v})$-DMSA has a potential clinical use for the detection of soft tissue tumors. The mechanism of the tumor uptake and the differentiation between inflammation and tumors will soon be continuing the research and undergoing clinical evaluation.

\section{SPACE 1647}

\section{Comprehensive MR imaging of spinal metastases}

Louis H. Wetzel (Presenter), Errol Levine, Solomon Batnitzky, A.W. Templeton; Kansas City, KS

Purpose: Oncology patients frequently present with ambiguous symptoms suggesting extradural spinal cord compression. This exhibit illustrates the value of "MR spinal survey".

Methods and Materials: A two-level body-coil study of the entire spine and spinal was performed on 67 patients with suspected cord compression. Patients with questionable lesions or obvious expansile metastases were further studied with appropriate spine-coil images.

Results: One-third had normal examinations, accurately excluding spinal cord or cauda equina compression. The MR spinal survey offers a fast, noninvasive method of screening oncology patients for significant spinal cord compression.

\section{SPACE 1648}

MR imaging of the foot: technique, normal anatomy, and pathologic conditions

Louis H. Wetzel (Presenter), Errol Levine, Mark D. Murphey, Stanton J. Rosenthal, A. W. Templeton, J. D. Bast; Kansas City, KS

Purpose: Evaluation of nontraumatic foot lesions with CT is often difficult because of the foot's complex anatomy and closely compressed soft tissues. This exhibit illustrates the use of MR imaging in evaluating foot lesions.

Methods and Materials: Examples are presented from a series of 21 surgically or biopsy-proved cases studied with MR imaging, including benign and malignant neoplasms, avascular necrosis, osteomyelitis, and abscess.

Results: The availability of multiple imaging planes and high soft-tissue contrast with MR imaging improved lesion detection and localization compared with CT. MR signal characteristics were helpful in characterizing hemangiomas and fibromatoses.

\section{SPACE 1649}

Comparison of MR imaging and bone scintigraphy for detection and evaluation of osseous spinal metastases

Louis H. Wetzel (Presenter), Diane E. Engelbrecht, Kirkman G. Baxter, Linda A. Harrison, A. W. Templeton; Kansas City, KS

Purpose: This exhibit compares MR imaging and bone scintigraphy in their ability to detect and evaluate osseous spinal metastases.

Methods and Materials: We reviewed bone scans and total spine MR examinations independently in 69 patients with various primary malignancies. The vertebral levels with metastases, as determined by each study, were recorded and assessed for lesion size on MRI and degree of activity on bone scan.

Results: MR imaging detected more metastases (575) than bone scans (392) in our initial study group and showed lesions in six symptomatic patients with negative bone scans. There was poor correlation of lesion size on MRI with activity on bone scan. Several large expansile metastases were not found by bone scan. MR imaging is clearly superior to bone scintigraphy for detection and assessment of osseous spinal metastases. Since bone scanning images the entire skeleton and costs less than MRI, it will continue to have a role in routine screening and follow up of patients at high risk for osseous metastases and those with stable metastases. However, for initial staging of patients prior to spinal radiation therapy and assessment of new spinal pain or neural compression symptoms, MR imaging should be performed.

\section{SPACE 1650}

Holmium-Yag laser for lumbar discectomy

Gerhard Wittich (Presenter), Ann Bjorkengren; Stanford, CA

Purpose: Percutaneous lumbar diskectomy is evolving as an alternative to surgical treatment of herniated lumbar disks. We present the result of in vitro studies aimed at developing a refined percutaneous delivery system for CT-guided laser diskectomy in patients with contained herniated lumbar disks. Methods: A holmium-yag laser with a peak power of $6 \mathrm{Kw}$ and a pulse duration of 350 microseconds was used for in vitro ablation of the nucleus pulposis of lumbar disks.

Results: These studies demonstrated the feasibility of ablation of disk material by laser energy. They have resulted in the design of a prototype delivery system for translumbar, CT-guided laser diskectomy.

Conclusions: Initial in vitro results suggest that a holmium-yag laser probe in conjunction with a refined delivery system may be clinically useful for percutaneous treatment of selected patients with herniated disks.

\section{SPACE 1651}

Correlation between magnetic resonance imaging, visual evoked potentials and clinical findings in multiple sclerosis

Ayça Altuğ (Presenter), Mefkûre Eraksoy, Ufuk Denizci, Hifzi Özcan, Sacit Karamürsel, Nuran Gökhan; Istanbul

MR examinations of 57 patients with definite or probable multiple sclerosis (MS) were evaluated to correlate the results with clinical and visual evoked potential (VEP) findings. Patients referred from different hospitals to the MS outpatient department of Istanbul University Medical Faculty between September 1989 and July 1990 underwent a complete neurological examination based on clinical signs and symptoms. Patients were classified by probability of MS as definite (34) and probable (23), by disease activity as acute, chronic with acute exacerbation or chronic progressive. The visual evoked potentials were obtained by the checkerboard method. MR examinations utilized a GE-CGR 0,5 Tesla unit in which $\mathrm{T} 1$ and $\mathrm{T} 2$ weighted images (WI) were acquired and the IR sequences were applied to demonstrate the brain stem and cerebellar plaques.

It was demonstrated that MRI was sensitive in $94 \%$ of the patients with clinically definite MS and $76 \%$ with clinically probable MS. There was a significant correlation between attack period and edema and conglomerate formation of the lesion, and no correlation between the clinical course, duration of disease, and the size of lesions. Cerebral atrophy did not correspond to the age of the patient.

VEP findings corresponded to clinical diagnosis, positive VEP results were obtained in 41 patients.

\section{SE}

\section{SPACE 1652}

\section{Neuroradiology (07)}

Magnetic resonance imaging of Sneddon syndrome

I. Muguerza, F. Aparisi (Presenter), R. Yaya, J.M. Pascual; Valencia

Sneddon syndrome (SS) is a very rare disease consisting of idiopathic diffuse livedo reticularis and cerebrovascular lesions. The SS is a very uncommon cause of stroke that affects mainly women in their middle age. The radiological findings of SS lies mainly in cerebral angiography and computed tomography (CT).

Purpose: We performed Magnetic resonance (MR) on ten patients with SS to compare the usefulness of MR findings with those of arteriography and CT in the diagnosis and follow up of the patients.

Methods and Materials: MR imaging was performed on a RM Max GE unit of $0.5 \mathrm{~T}$. T-1 and T-2 weighted axial images were studied.

Results: On MR three types of lesions were found: 1. large and medium size infarctions in the territory of middle and posterior cerebral arteries. These lesions were seen as focal areas of low density on CT, and as intravascular occlusion on cerebral angiography. 2. Diffuse cerebral cortical atrophy, usually seen on CT. 3. A special MR finding was multiple areas of increased signal intensity in subcortical area, in the deep white matter and in the posterior fossa. These lesions were not evident on CT and angiographic studies and were probably caused by microinfarctions. MR is the method of choice for the study and evaluation of patients with SS. 


\section{SPACE 1653}

MRI imaging of spinal neurinomas, a study of $\mathbf{3 0}$ operated cases D. Balériaux (Presenter), J. Widelec, W. O. Bank, D. Krause, J. L. Drape, J. L. Dietemann, J. Tongio; Bruxelles/Strasbourg

Purpose: The aim of this multicentric study was to evaluate MRI's ability to diagnose spinal neurinomas in $\mathbf{3 0}$ subsequently operated cases.

Methods and Materials: The spinal locations were as follows: 7 cervical, 5 thoracic and 18 at the lumbar level. An MR Max $0.5 \mathrm{~T}$ imager was used in 17 cases and a Gyroscan $1.5 \mathrm{~T}$ or $0.5 \mathrm{~T}$ in 13.

The sequences used were: GE or SE T1 (fast), T2 and gadolinium enhanced T1. Results: MRI showed well-limited lesions in 22 cases. In comparison to normal spinal cord, T1 neurinomas signals were hypo- $(14 x)$ or isointense $(16 x)$. All neurinomas were hyperintense on T2; they exhibited heterogeneous appearances at the lumbar level $(10 \times)$, which were confirmed on gadolinium-enhanced T1 sequences. Significant enhancement was constantly observed.

Extraspinal locations were infrequent $(3 \times)$; hourglass neurinomas were found $(5 \times)$. In investigating intradural tumors $(22 \times)$, supplementary axial sections were necessary to determine their relationship to the cord of cauda equina nerve roots.

Conclusions: In addition to the classic diagnostic pitfall of dorsal meningiomas, the authors emphasize the possible variable appearances of lumbar neurinomas: other medullary tumors need to be ruled out.

\section{SPACE 1654}

The radiologic features of nonosseous causes of stenosis of the lumbar spinal canal

Solomon Batnitzky (Presenter), Hilton I. Price, Donald A. Eckard, A.W. Templeton; Kansas City, KS

Purpose: Spinal stenosis is an important cause of low back pain. The most commonly encountered causes of spinal stenosis are osseous and are either congenital or, more commonly, associated with degenerative spinal disease. Less emphasis has been placed on the nonosseous causes of spinal stenosis. Methods and Materials: This exhibit illustrates and discusses the clinical, CT, MR, and myelographic features of nonosseous causes of stenosis of the lumbar spinal canal.

Results: These include lymphoma, myeloma, metastases, abscess, thickened ligamentum flavum, and arachnoiditis. Less common causes are epidural lipomatosis, extramedullary hematopoiesis, and transient physiologic constriction of the subarachnoid space by epidural venous distention.

\section{SPACE 1655}

Cerebral demyelination mimicking intracranial neoplasm

Solomon Batnitzky (Presenter), Donald A. Eckard, J.Kepes, A.W. Templeton; Kansas City, KS

Purpose: Cerebral demyelination can present as large mass-like lesions and can therefore mimic brain tumors.

Methods and Materials: These lesions may be solitary or multiple and are frequently seen in young adults. Since the clinical presentation may be insidious and CT and MR examinations often show large lesions with peripheral enhancement, these are frequently mistaken for intracranial neoplasms, such as astrocytomas. Other frequent misdiagnoses include metastases and infection.

Results: From an experience of over 30 cases of mass-like areas of demyelination, representative examples will be presented. CT, MR, and pathologic features will be demonstrated.

\section{SPACE 1656}

\section{Neurocysticercosis CT and MR imaging}

Carlos Bazan III (Presenter), George Ripeckyj, Kathleen Kagan-Hallet; San Antonio, TX

Neurocysticercosis is a parasitic infestation of the CNS that is common in some parts of the world (Mexico, Portugal, Spain, Central America, Eastern Europe, Africa, and Asia). Increased travel to and from endemic areas has resulted in an increasing number of neurocysticercosis cases being diagnosed in non-endemic areas. The purpose of this exhibit is to review and compare imaging modalities in the evaluation of neurocysticercosis.

Methods and Materials: Cases of neurocysticercosis were obtained retrospectively from CT, MRI, and surgical pathology records.

Results: In our clinical setting most patients with neurocysticercosis were initially evaluated with CT, usually after presenting with seizures. The presence of multiple intracranial calcifications suggested the diagnosis of neurocysticercosis. CSF immunologic studies and/or pathologic examination of resected lesions confirmed the diagnosis. CT was superior to MRI in detecting the calcifications of cysticercosis. MRI was superior to CT in demonstrating the non-calcified lesions of cysticercosis whether parenchymal, intraventricular, or subarachnoid in location. MRI could identify intraventricular lesions without the need for intraventricular contrast. Gadolinium enhanced images were particularly useful for demonstrating active lesions.

\section{SPACE 1657}

Granulometric analysis of spherical and non-spherical embolization particles

R. Beaujeux (Presenter), A. Laurent, L. Domas, M. Wassef, J. J. Merland; Paris

We have carried out the granulometric analysis of different embolization materials by the mean of two methods: optical microscopy and semi-automatical shape measurement. We have measured the size of particles according to two dimensions and determined an index adapted to the embolization particles.

We have studied following particles:

- non spherical: dura mater, gelfoam, PVDF, polyvinyl alcohol

- spherical: polystyrene, dextran, silicone, acrylic beads

The a. $\mathrm{m}$. particles have been obtained by different fabrication methods which will influence the granulometric distribution.

The granulometry of the non-spherical particles (obtained by mechanical fragmentation) is wider than advertised on the commercial leaflet and frequently tends to concentrate on the minimal dimension.

On the opposite the spherical particles are well calibrated, and especially the acrylic beads which we have been able to divide into very narrow granulometric peaks.

\section{SPACE 1658}

Dynamic computed tomography: kinetics of non-ionic contrast media in brain metastasis of lung cancer

Bruno Betsch (Presenter), Michael V. Knopp, Udo Trost, Stefan Delorme, Gerhard van Kaick; Heidelberg

Purpose: The optimal timing for cerebral CT after i.v. administration of non-ionic contrast media for detection of brain metastasis is still controversely discussed. To provide a rational basis the kinetics of non-ionic contrast media in brain metastasis of lung cancer has been studied.

Methods: The kinetics of non-ionic contrast media ( $100 \mathrm{ml}, 62 \%$ lopromide) after intravenous bolus administration (Flows: $40 \mathrm{ml} / \mathrm{min}$.) in brain metastasis of bronchial carcinoma has been studied with dynamic computed tomography. The pharmacokinetic calculations and the compartiment-model analysis have been performed separately for blood, brain tissue and metastasis.

Results: In normal brain tissue the kinetics of non-ionic contrast media can often be described best with a 1 -compartiment model (Maximum shortly after administration - monophasic decline of tissue iodine concentration).

In brain metastasis the kinetics of non-ionic contrast media can frequently be described best with a 2-compartiment model (Maximum shortly after administration - biphasic decline of tissue iodine concentration).

Conclusions: Non-ionic contrast media shows a different pattern of kinetics in brain metastasis compared with normal brain tissue. Considerable differences already appear in the early phase after administration. The ongoing investigation will clarify whether these differences are suitable for a rapid and sensitive detection of brain metastasis shortly after administration of non-ionic contrast media especially using modern, fast cycle CT-systems.

\section{SPACE 1659}

Magnetic resonance imaging in the early assessment of spinal injuries Roger Bodley (Presenter). Ali Jamous, Debbie Short, Philip Savage: Aylesbury

Purpose: To assess the role of Magnetic Resonance Imaging (MRI) of the spinally injured patient in the acute phase of injury.

Methods: All new acute admissions to the National Spinal Injuries Centre at Stoke Mandeville Hospital underwent MRI of the affected part of the spine within five days of injury and within 24 hours if possible. Using a modified frame enabling continuous cervical traction during imaging where appropriate, patients were assessed using a Hitachi MRP 20 Permanent Magnet System with $\mathrm{T} 1$ and $\mathrm{T} 2$ weighted sequences and Gadolinium enhancement if necessary.

Results: Excellent images of the damaged spinal cord and surrounding tissues were obtained. Several patterns of cord injury were seen, ranging from complete disruption through focal altered signal changes to apparent normality. In several cases appreciation of the state of adjacent intervertebral discs and of focal changes in the cord that may herald the onset of syringomyelia led to a change in the operative strategy.

Conclusions: MRI in the acute stage of spinal cord injuries is feasible and useful. It allows rational decision on early management to be made and it is crucial if surgery is contemplated. 


\section{SPACE 1660}

\section{Degenerative disease of the spine: a review}

P. Coscojuela (Presenter), J. Ruscalleda, M. de Juan, E. Guardia; Barcelona

Purpose: Lumbar pain and back pain are mainly related to discal pathology and many degenerative processes may be involved. The different phenomena implied are discussed.

Methods and Materials: We reviewed X-Ray studies, myelographies, CT and $M R I$ of a large series of patients with degenerative disease of the spine and low back pain. The structural degenerative changes were evaluated and we describe the different degenerative processes affecting cartilaginous, synovial and fibrous articulations and also their complications: listesis, alineation alteration, discal hernia, canal stenosis. We describe these progressive changes and the most appropriate studies for diagnosis.

Results: Degenerative disease of the spine is a biologic process generally appearing in the $3^{\text {rd }}$ decade of life. It's often difficult to differentiate from pathologies which give rise to low back pain. Clinical signs and symptoms should be related to the radiologic image and diagnosis should be made globally.

\section{SPACE 1661}

Tc-99m-HMPAO-Spect in patients with reversible cerebrovascular disease (CVD): comparison of the sensitivity without and with Acetazolamid

W.-H. Dingler (Presenter), D. Beil, H.-K. Deininger; Darmstadt

SPECT imaging of the brain was performed in 66 patients with reversible CVD utilizing Tc-99m-HMPAO; 35 patients were studied after intravenous administration of $1 \mathrm{~g}$ Acetazolamid, 31 patients got none. Sensitivity of these two methods were compared differently in reversible hemispheric (TIA) and vertebrobasilar insufficiency (VBI). Cerebral infarction was excluded by TCT. Sensitivity in TIA without provocation was $55 \%$, in VBI $36 \%$. Acetazolamid improved sensitivity to $90 \%$ and $64 \%$. In reference to total CVD sensitivity increased from $48 \%$ to $80 \%$. II patients who were investigated with both methods within a few days, showed an increase of sensitivity from $45 \%$ to $81 \%$. 3 patients with amaurosis fugax and severe stenosis of the internal carotid artery were normal with Acetazolamid. However, in 8 patients with asymptomatic severe carotid stenosis the ipsilateral hemispheric blood flow was reduced by the provocation test.

Conclusions: SPECT imaging with Tc-99m-HMPAO shows in patients with reversible CVD in provocation with Acetazolamid a distinct higher sensitivity.

\section{SPACE 1662}

\section{A retrospective analysis of spinal tumors by MRI}

John J. Eick (Presenter), John A. Kalmar, Kenneth D. Miller, KelleyP. Douglas, Roger H. Tutton; New Orleans, LA

Magnetic Resonance Imaging is an established and proved diagnostic modality for evaluation of the spine and spinal cord. The subsequent introduction of contrast material for MRI has further increased the sensitivity and specificity of the modality for spinal tumor evaluation. Our MRI experience began in 1985 and a review of our spine material revealed more than 250 patients with tumors involving the osseous spine or spinal contents. A retrospective analysis of our spinal tumor cases was performed and our exhibit presents the data.

The majority of spinal tumor cases were found to be metastatic lesions to bone with metastatic carcinoma from tumors of the lung, breast and prostate being the most common. The most frequently encountered primary tumors of the spine were astrocytoma, ependymoma, neurogenic tumors, meningioma and lipoma. Representative examples of both the common and uncommon lesions with discussions are presented.

\section{SPACE 1663}

\section{Spontaneous subarachnoid hemorrhage with negative angiography} M. T. Farres (Presenter), H. Ferraz-Leite, E.Schindler, M. Mühlbauer; Vienna

In subarachnoid hemorrhage ( $\mathrm{SAH}$ ), angiography reveals no source of the bleeding in about 15 to $35 \%$ of cases, as has been shown in the literature. In order to find out whether a certain SAH distribution - as seen on CT - corresponds to negative angiograms or not, the neuroradiological findings in 28 patients with SAH were evaluated. In all cases SAH was confirmed with CT, but the angiograms showed neither aneurysm nor angioma. In 20 cases angiography was repeated, and three patients had a third angiographic study (with re-angiography, an aneurysm was ascertained in two patients). In most cases CT showed but a small amount of blood within the prepontine and/or the interpeduncular cistern, whereas hemorrhage in one or both Sylvian fissures was found in four cases only. Nearly all patients with prepontine or interpe- duncular SAH fully recovered. Patients with other SAH distribution had hypertension and/or angiographic findings pointing to arteriosclerosis.

Based on our study, it is suggested that SAH confined to the prepontine or to the interpeduncular cistern may be rather seldom caused by an aneurysm. Hence, if CT shows such a pattern of SAH and the angiograms are negative, the question is raised, whether or not a second angiographic study should be carried out.

\section{SPACE 1664}

Studies of the hypophysis of patients with primarily aldosteronism of postoperative treatment by MR imaging

I. D. Fedina (Presenter), N. M. Chikhladze, S. K. Ternovoy; Moscow

All studies were performed using MR Imager (Bruker, FRG). The state of the hypophysis was studied in 25 patients (pts) with primarily aldosteronism (PA) after adrenalectomy: 16 pts with residual arterial hypertension (RAH) and 9 pts with normal arterial pressure (NAP). The comparison group consisted of 19 pts with hypertension disease (HD). In 16 pts with residual arterial hypertension (RAH) only 11 pts showed hyperintensive sections in different parts of the hypophysis, unevenness and diffusion of the contour of the hypophysis in 9 pts with normal arterial pressure (NAP) only 4 pts showed analogous changes. All patients with PA had normal sizes of the hypophysis and the unevenness of its structure. In $19 \mathrm{pts}$ with HD only 7 pts showed the heterogeneity of the structure, and 3 pts had hyperintensive sections. Visualized by MR Imaging the changes of the substance of the hypophysis have proved the necessity of the further studies of the morphological and functional state not only of the adrenal glands but also of the hypophysis in patients with PA and especially in patients with residual arterial hypertension.

\section{SPACE 1665}

The role of plain and Gadolinium enhanced MR in the evaluation of spinal cauda neoplasms

M. Gallucci (Presenter), C. Masciocchi, A. Bozzao, A. Barile, A. Cifani, R. Passariello; L'Aquila

The purpose of the study was to evaluate MR effectivity in the diagnosis and characterization of the neoplasms of the cauda equina. Twenty-three subjects suffering from cauda tumors were evaluated by plain and enhanced MR. Other examinations were acquired in 15 cases (myelography, CT and CT-myelography). In all the cases surgical procedures were performed and pathological evaluation obtained. There were 5 neurinomas, 7 meningiomas, 4 epidermoids and 7 ependimomas. MR proved to be useful in the diagnosis of all the cases examined although it was not always able to differentiate the neoplasms each other. Nevertheless differential patterns mostly based on location and type of enhancement were identified on MR.

\section{SPACE 1666}

\section{Magnetic resonance and microprolactinoma}

E. Gomez (Presenter), M.P. Sanz, A. Capdevila, J. Aldoma, P. Martinez, J. Gili, M. Baquero; Barcelona

We have studied a 100 patients, who all had high plasmatic values of prolactin. In $50 \%$ of them MR was positive and we were able to see the lesion. $25 \%$ of the cases we couldn't see any abnormalities in the hypophysis. We used $\mathrm{Tl}$ weighted images (TR $=400-600 \mathrm{~ms} / \mathrm{TE}=20 \mathrm{~ms}$ ) on all of them, and T2 weighted images $(T R=2000-2500 \mathrm{~ms} / \mathrm{TE}=30-100)$.

In 25 patients the study was performed after administration of Gd-DTPA. We present the tables of the morphological findings, which allow us the diagnosis of the different sequences carried out, as well as the behaviour of lesion shown after contrast administration.

The best information was obtained on coronal plane in Tl weighted images after administration of Gd-DTPA.

\section{SPACE 1667}

\section{MR-scoring in craniocerebral trauma}

A. Janousek (Presenter), J. Poigenfürst, G. Siakos; Vienna

Aim of this study is to show the prognostic value of Ultra Low Field-Magnetical Resonance (ULF-MR) Tomography in craniocerebral trauma.

Since June 1987 Lorenz Böhler Trauma Center has been using an ULF-MR with a magnetical field-strength of .02 Tesla. More than 800 investigations in 600 patients with craniocerebral trauma were performed. For clinical estimation Glasgow Coma Scale (GCS) was used, the results were correlated with MR-findings.

ULF-MR shows a high sensitivity in the noninvasive diagnosis of haematoma and contusion in craniocerebral trauma. Clinical results show partial correlation with $M R$.

MR findings may improve clinical scores. A possible new MR-score is presented in this paper. 


\section{SPACE 1668}

Biokinetic of the benzodlazepine antagonist Ro 16-0154 (lomazenil) in man

Uwe Keske (Presenter), J. Hierholzer, M. Cordes, A. Debrand-Passard, H. Eischstädt, R. Felix; Berlin

Purpose: The benzodiazepine-antagonist Ro 16-0154 (J123-Iomazenil ${ }^{\odot}$, Mallinckrodt) was introduced for SPECT-imaging of the brain. In order to optimize the acquisition protocol, its biokinetic was evaluated.

Patients and methods: Via sequential scintigraphy 0-90 min after injection of $120 \mathrm{MBq}$ J123-Iomazenil, time-activity-curves of brain, heart and liver were calculated in 30 patients. Multiple plasma and urine samples were taken.

Results: The maximum brain uptake of lomazenil is reached after 17.8 minutes. Afterwards, a linear decrease with an average of $5.6 \%$ per $10 \mathrm{~min}$ is noted. The maximum plasma-concentration is found after a few seconds and is followed by a fast and exponential decrease (63\% in $120 \mathrm{~min}$ ). An average of $63 \%$ of the injected activity is found in the urine after $90 \mathrm{~min}$. A smaller part is excreted by the liver.

Conclusions: An acceptable noise-background-ratio for SPECT-gamma camera images of the brain is found $30 \mathrm{~min}$ after injection. This does not significantly change up to $150 \mathrm{~min}$ p.i. When long SPECT-acquisition times are chosen, it has to be considered that the brain-lomazenil-concentration decreases with $5.6 \%$ per $10 \mathrm{~min}$. Sequential scintigraphy of the brain with Iomazenil enables quantification of the global benzodiazepine-receptor kinetic of the brain.

\section{SPACE 1669}

Experimental studies and improvement of biocompatible new embolization microspheres

A. Laurent (Presenter), R. Beaujeux, M. Wassef, J. J. Merland, E. Boschetti, M. Brouard, G. Leconte; Paris

Classical embolization particles present a lot of problems due to their irregular shape (bad calibration) and to the kind of the polymer.

The authors describe a new type of embolization materials; acrylic microspheres which are synthetized by reversed emulsion.

Experimentation has been practised on Sprague Dowley rats to check out on biocompatibility.

The materiel improvements are following:

- adaptation of the synthetis techniques as to obtain a complete range of diameters $(150-1200 \mu)$

- adaptation of the microsphere surface as to boost the cellular adhesion

- division into different calibers by the mean of watered sieves which allow us to obtain very narrow peaks of granulometry $( \pm 50 \mu, \pm 100 \mu)$

- polymer coloration

These microspheres present many advantages and allow accurate targeting of the embolization.

\section{SPACE 1670}

Optimized MRI-sequences and parameters to avoid pitfalls in detection of discus prolaps of the spine

Th. Lehnen (Presenter), E. G. Eising, R. W. Dalicho; Recklinghausen

MRI is known as an important diagnostic procedure to clear up discus prolaps of the spine. In spite of using contrast media, pitfalls might result of scarring in postoperative patients or in cases of lateral protrusions or small sequestrated masses.

To avoid diagnostic errors in such cases, we have tried to optimize MRI-parameters (e.g. imaging-sequence, TE, TR, slice-orientation, time of application of contrast medium) in about 50 patients with such problems.

\section{SPACE 1671}

Magnetic resonance imaging in pedlatric neuromuscular disorders Gin-Chung Liu (Presenter), Yuh-Jyh Jong, Chi-Hsiun Chiang; Kaohsiung

Purpose: Differentiation of different types of pediatric neuromuscular disorders by Magnetic Resonance imaging (MRI).

Methods: Totally 38 cases [Duchenne's muscular dystrophy (DMD) 15, spinal muscular atrophy (SMA) 10, dermatomyositis/polymyositis (DM/PM) 4, congenital myopathy (CM) 2, arthrogryposis multiplex congenita (AMC) 2, myotonic dystrophy (MD) 1 , lipid storage myopathy (LSM) 1, mitochondrial myopathy (MM) I, Pompe's disease 1 and myasthenia gravis (MG) 1] were investigated with a $0.5 \mathrm{~T}$ superconductive scanner. $\mathrm{T} 1$ - and $\mathrm{T} 2$-weighted spin echo sequences in axial and coronal projections of calf and thigh muscles were obtained. For SMA and AMC, evaluation of cervical cord with same techniques were also performed.

Results: MRI clearly demonstrated the abnormality of subcutaneous fat and muscular bundles with its advantages of multiplanar capability and excellent tissue contrast. The findings of MRI are: a) DMD: preserved muscular groups of gracilis (15/15), sartorius (12/15), semitendinous (9/15). b) SMA: increased subcutaneous fat in all subtypes with ragged islands of muscular tissue within a large mass of panniculus adiposus in intermediate type. c) acute stage of DM/PM: high T2-signal intensity. d) CM: partly preserved semimembranous and biceps muscle. e) LSD: increased subcutaneous fat. f) AMC: severe fatty degeneration. g) Pompe's disease: preserved muscle with mild degeneration. $h$ ) MD and MG: normal.

Conclusions: MRI provides a complementary study and guidance of muscular biopsy for differential diagnosis of neuromuscular disease and can serve as a tool for follow up study of disease progression or treatment response.

\section{SPACE 1672}

A new CT method for demonstrating the site of CSF leakage M. N. H. Lloyd (Presenter), P. M. Kimber, E. H. Burrows; Southampton

Accurate definition of the site of CSF leakage is essential for successful surgical management of CSF rhinorrhoea and associated recurrent meningitis. Previous imaging methods, including conventional CT, have been disappointing.

We use a dedicated high-resolution CT technique comprising fine slices in coronal and sagittal planes with a bone algorithm for data manipulation. The site of the responsible communication between cranial cavity and sinus is well demonstrated and additional features of adjacent intracranial air bubbles or adjacent sinus soft-tissue mass are frequently shown.

The technique is described and the result obtained in 20 patients are discussed.

\section{SPACE 1673}

\section{CT-guided stereotaxy}

L. Mistina (Presenter), M. Galanda; Banská Bystrica

CT-stereotactic interventions are used for evacuation of brain hematomas, abscesses, biopsy, interstitial radiotherapy, but are not so common in functional stereotactic neurosurgery.

We present our experience with CT (Siemens) calculation of the target area for stereotaxy (Fischer instruments) in the VL thalamus, the brachia conjuctiva cerebelli (the motor disorders) and periaqueductal gray (intractable pain), where the electrodes for permanent stimulation (Tesla neurostimulating devices) were implanted. After neurophysiological control CT examination confirms the precision of positioning of the electrodes in the target, even applying Multiplanar reconstruction.

The advantages and the drawbacks of CT-stereotaxy are discussed.

\section{SPACE 1674}

Evaluation of regional cerebral blood flow in schizophrenia using TC-99m HM-PAO SPECT

S. Mizumura (Presenter), T. Oishi, R.Saito, H. Hayashi, S. Takahashi, A. Okuyama, T. Kumazaki, Y. Yamagishi, K. Ebata; Tokyo

Purpose: To evaluate regional cerebral blood flow (rCBF) distribution in schizophrenia using Tc-99m hexamethyl-popyleneamine oxine (HM-PAO) SPECT by both qualitative and quantitative methods.

Methods and Materials: Eleven patients with schizophrenia and 7 healthy volunteers were studied. Ten minutes after the injection of $740 \mathrm{MBq} T \mathrm{~T}-99 \mathrm{~m}$ HM-PAO, SPECT images were taken using a rotating gamma camera system. Regions of interest (ROIs) were drawn on the imaging of bilateral frontal, temporal, occipital, parietal cortex, basal ganglia and cerebellar hemisphere. The rCBF distribution in schizophrenia was evaluated by the mean value activity of all pixels within each ROI and was compared with that of healthy volunteers.

Results: The rCBF distribution in schizophrenia was obviously different from that in healthy volunteers. There were variable patterns of $\mathrm{rCBF}$ distribution e.g. hypofrontality, asymmetric accumulation and crossed cerebellar diaschisi in patients with schizophrenia. In 5 cases the rCBF distribution has been markedly improved during a long term follow up treatment.

\section{SPACE 1675}

Cerebro-meningeal tuberculosis (CMTB) and acquired immunodeficiency syndrome (AIDS)

L. Muñoz (Presenter), J. de la Torre, M. F. Villoria, F. Fortea, C. Iglesias, A. Vega, Y.C. Benito; Madrid

Purpose: We have revised 194 patients with AIDS and neurologic complications, studied by CT and MRI. 35 of these patients were diagnosed of CMTB by CSF Ziehl staining or culture. This incidence is higher than the ones published in other series. Our purpose is to investigate if there exists some difference in the radiological findings in CT or MRI between patients with AIDS and non-immunosuppressed patients.

Methods and Materials: We have performed $C T$ in all patients, with i. v contrast (iohexol), and MRI in three of them, following i.v. paramagnetic 
contrast injection (gadolinium DTPA).

Results: We found hydrocephalus (51\% of the patients), meningeal enhancement $(48 \%)$, parenchymatous lesions $(34 \%)$ and vascular complications $(23 \%)$. Imaging studies were normal in $23 \%$ of the patients. We have not found significant differences between AIDS and non-AIDS patients. The more frequent CT feature was non-obstructive hydrocephalus associated in $70 \%$ of the cases to meningeal enhancement. This finding is opposed to the one reported by other authors. This justified the absence of meningeal enhancement by a supposed lower immunedefense capability in AIDS patients. Finally, MRI has been very useful in showing meningeal enhancement in three patients with normal CT studies, providing a more precocious diagnosis and proper treatment.

\section{SPACE 1676}

MRI in endovascular treatment of cerebral arteriovenous malformations L. Prayer (Presenter). D. Wimberger, J. Kramer, R. Stiglbauer, B. Richling, G. Bavinski, Ch. Matula, H. Imhof; Vienna

Purpose: To determine the usefulness of MRI in assessing embolization related changes of cerebral AVMs.

Patients and methods: 31 patients with angiographically proved cerebral AVMs were studied by MRI posttherapeutically. Pretherapeutical examinations were available in 20 patients. T1- and T2-weighted SE-sequences were performed in at least two imaging planes on a superconducting magnet operating at 1,5 Tesla.

Results: AVMs could be demonstrated excellently by MRI before and after embolization therapy. Complete thrombosis was seen in 5, partial thrombosis in 26 patients. Posttherapeutically, ischemic infarction and/or small hemorrhage were noticed in 8 patients. Changes of the nidi assessed by MRI correlated with angiographical reports in all patients.

Conclusions: MRI is a valuable non-invasive tool in assessing embolizationrelated changes of both cerebral AVMs and surrounding brain tissue.

\section{SPACE 1677}

Application of flow-colour images to the cerebral angiography study J.M. Pumar (Presenter), M. Alvarez, J. Lado, P. Martinez, J. Vidal, M. Martinez, J. Pereira, F. Hermida; Santiago de Compostela

Purpose: The purpose of this research work is to analyse the application of "flow-colour" images as an addition to the cerebral angiography study in different pathologic processes.

Methods and Materials: We have made a study by means of using "flow-colour" images in the cerebral angiography of two different groups of patients. The pseudo-colour scale assigns a colour to every single pixel in the image, at any moment in the sequence, depending of the maximum concentration of contrast. Within group I we examined 50 patients with normal cerebral angiography, and the second group was formed by 30 patients with different types of cerebral pathology: arteriovenous malformations, vascular obstructions, aneurysms, meningiomas and some other cerebral tumors. We have studied the differences and variations appearing in the "flow-colour" cerebral angiography map of the different pathologies in relation to the normal group.

Results: The application of "flow-colour" images in cerebral angiography offers additional information in different groups with intracranial pathology, specifically in vascular processes.

\section{SPACE 1678}

Therapeutic embolization of cerebral aneurysms with microcolls J.M. Pumar (Presenter), L. Guimaraens, M. Alvarez, J. Vidal, A. Perez Batallon, P. Martínez, J. Lado; Santiago de Compostela

Purpose: The purpose of this research work is to give detailed description of the embolization technique in cerebral aneurysms. We pay special attention not only to the indications and counterindications of the technique, but also to the attention and care given to the patient in pre- and post-embolization periods.

Methods and Materials: We make a femoral puncture by inserting a $5 F$ catheter as far as the origin of the carotid artery. Then, we make a carotid direct puncture by means of which a TRAKER-18 is introduced until it penetrates the aneurysm. When this is done, we insert Complex Helical Platinum Coils by means of a PULSER 16 trying to occupy about the $70 \%$ of the lumen of the aneurysm. During the exploration, we make several angiographic controls using the femoral catheter.

Results: Having performed this clinical experience, we consider that the embolization technique with microcoils should be taken into account as a method to treat aneurysms, especially when they are pediculated or giant.

\section{SPACE 1679}

\section{CT and Intraoperative ultrasound in cerebral tumours}

Ewa Zajac, John Koivukangas, Juhani Pyhtinen (Presenter); Oulu

Intraoperative ultrasound imaging (IOUS) has been used at our center for cerebral tumour surgery control since 1982 .

Purpose: To compare CT and IOUS findings in cerebral tumours.

Methods: IOUS was performed with 5-7 MHz linear and sector transducers during craniotomy and these findings were compared with CT findings in 22 consecutive adult cerebral tumours operated during 1987-88. These tumours consisted of 9 astrocytomas grade 1-2, 5 astrocytomas grade 3-4, 2 oligodendrogliomas, 4 meningeomas and 2 metastases.

Results: Nonuniform contrast enhancement was associated with similarly nonuniform echogenicity. Calcifications in 6 cases could be found by both methods. IOUS was found to better differentiate cystic parts of the tumours. Grade I-2 astrocytomas which were non-contrast enhancing uniformly hypodense lesions on CT showed up as uniformly hyperechogenic lesions in IOUS. Corresponding diameters of the tumours showed high statistical correlation.

Conclusions: IOUS serves to localize and demarkate subcortical tumours, especially grade 1-2 astrocytomas, with most tumour features (size, cysts, calcifications, necroses) being similar in both CT and IOUS and in certain cases, IOUS gives critical supplementary information.

\section{SPACE 1680}

\section{Evaluation of the peripheral nerves (PN) and their lesions by ultrasound (US)}

S. A.A. Qaiyumi (Presenter), H. Milbradt, S. Hussein, K. Weissenborn, B. Frank, M. Prokop, M. Galanski; Hannover

Thus far, imaging of PN has been rather limited. Our study was designed to evaluate the diagnostic value of US in PN of the lower and upper extremities. Methods and Materials: We examined 20 healthy volunteers and 43 patients with various pathology to evaluate imaging features of normal and pathological conditions. We used Sonoline AC/SL US-machines (Siemens) and $7.5 \mathrm{MHz}$ and $5 \mathrm{MHz}$ transducers.

Results: PN are hyperechoic bands with parallel internal structures and an echogenicity that is strongly dependent on the tilt angle of the transducer. All major PN could be demonstrated with exception of sural and peroneal nerves. Neuroma $(n=10)$ were almost round and hypoechoic and neurinoma $(n=4)$ were often oval and hypoechoic. Neurofibroma $(n=4)$ were oval or round and hyperechoic. Neurofibrosarcoma $(n=2)$ were irregular, hypoechoic and inhomogeneous. Focal morphological changes could be found in following cases: carpal tunnel syndrome $(n=10)$, ulnar sulcus syndrome $(n=4)$, lipomatosis $(n=2)$ and amputation of radial nerve $(n=1)$. Compression of the nerves by surrounding masses were seen in 6 cases.

Summary: US proved to be an easy, non-invasive and effective method to examine PN and their lesions.

\section{SPACE 1681}

Value of IV paramagnetic contrast (Gd-DOTA) injection in lumbar disk and nerve root pathology of the non-operated spine

B. Roger (Presenter), C. Grataloup, D. Folinais, J.P. Benazet, M. LavalJeantet; Paris

a) Aim of the study: The authors present 5 cases of lumbar MRI representing 5 different typical diagnoses and raising problems of differential diagnosis or choice of treatment: foraminal L4-L5 disk hernia, extruded L5-S1 disk hernia, posterior articular synovial cyst of L5-SI, radicular cyst, intradural neuroma of $\mathrm{L} 3$ and foraminal neuroma.

b) Methods: These 5 patients underwent $\mathrm{T1}$ - and $\mathrm{T} 2$-weighted sequences after intravenous injection of paramagnetic contrast agent (Gadolinium-DOTA, i.e. Dotarem ${ }^{\oplus}$, Guerbet) in the sagittal and transverse planes.

c) Results: This study analyses and compares, for each lesion, the site, appearance and variation in the signal in order to define the MRI signs. The post-contrast sequence was found to be essential for the demonstration and characterisation of the lesion.

d) Conclusions: A summary table is proposed as a function of the variation in the signal, allowing the positive diagnosis and differentiation of each of the aetiologies presented.

\section{SPACE 1682}

Acquired and congenital abnormalities of the cerebral cortical pattern: differential diagnosis by MR

A. Splendiani (Presenter), M. Gallucci, A. Bozzao, F. Caramia, O. Migliori, A. Cifani, R. Passariello; L'Aquila

$M R$ is the examination of choice in the evaluation of the abnormalities of cortical architecture because of the clear distinction between the gray and 
white matter and the ability to generate images in a variety of planes. These imaging attributes are useful in identifying and learning about the spectrum of congenital cerebral abnormalities that arises from disturbances in neuronal migration. These pathologies are often not-differentiable from encephaloclastic acquired disturbances. The purpose of our study was to identify the criteria that permit a correct differential diagnosis between malformative and acquired pathologies. We included in the study 61 subjects correlated by MR examinations in which cortical abnormalities were demonstrated. In 30 cases the pathologies were encephaloclastic. In the remaining 31 cases malformative (7 schizencephaly, 6 polymicrogyria, 9 pachygyria, 9 neuronal heterotopia). In 15 cases there was surgical confirmation.

MR resulted to be the examination of choice able to recognize and characterize the disorder, giving a satisfactory differential diagnosis, in order to better assess the prognosis, the therapeutic approach and the prevention.

\section{SPACE 1683}

Rheumatoid arthritis of the occipito-cervical region: quantitative and qualitative assessment by Gd-enhanced MRI

M. Stiskal (Presenter), A. Neuhold, W. Leeb, J. Smolen, L. Wicke; Vienna

Purpose: Aim of the study was to define the importance of Magnetic Resonance (MRI) for patients with clinically and laboratory confirmed rheumatoid arthritis, especially to evaluate the significance of Gd-DTPA enhancement of the pannus in the occipito-cervical region.

Methods and Materials: We examined 90 patients with rheumatoid arthritis and neurological symptoms. All investigations were done with a $0,5 \mathrm{~T}$ superconductive unit. Sagittal and coronal planes $5 \mathrm{~mm} \mathrm{SE}$ technique, short TR/TE sequences $(450 \mathrm{msec} / 30 \mathrm{msec})$ before and after Gd-application, $6 \mathrm{~mm}$ slices gradient echo technique ( $555 \mathrm{msec} / 40 \mathrm{msec}$, puls. ang 20 ) were obtained. The patients were categorized according to the morphology and signal intensity of the pannus as seen on unenhanced scans and further classified their enhancement status into three groups.

Results: MR-morphology reflects the different phases of the inflammatory process at the odontoid level. 30 patients in whom a clearcut enhancement of the pannus was noted received a combination therapy (non-steroid antirheumatic agents, low dose cortisone). 3 months follow-up examinations showed a quantitative and qualitative significant decrease of signal intensity of the pannus tissue as well as a normalization of the laboratory values. In the other 20 patients signal of the pannus was still high also the laboratory values pathologically elevated.

Conclusions: Our results show a possible positive association between enhancement status of pannus tissue in chronic rheumatoid arthritis patients and pathological serological values reflecting the activity status of the disease.

\section{SPACE 1684}

\section{MR findings of hypoxic brain damage}

Satoru Tazawa (Presenter). Shoki Takahashi, Kiyoshi Ishii, Kou Matsumoto, Kiyohiko Sakamoto, Makoto Takahashi, Mikio Suzuki; Sendai

\section{Purpose: Evaluation of hypoxic brain damages on MR images.}

2. Methods and Materials: T1- and T2-weighted images and contrast enhanced $\mathrm{Tl}$-weighted images were obtained in 5 patients with hypoxic brain damage with $1.5 \mathrm{~T}$ and $0.5 \mathrm{~T}$ MR systems. Patients included were two who had cardiac arrest, two who had systemic hypotension and the other who had hypoglycaemia.

3. Results: On MR images, diffuse brain swelling progressed from 3 days to 12 days after the ictus, and it subsided after 2 weeks. T2-weighted images showed hyperintense lesions in the cerebral cortex. From 10 days to 2 months after onset, $\mathrm{Tl}$-weighted images with and without contrast medium showed findings which suggested diffuse laminar necrosis of the cerebral cortex. Distribution of such lesions was most prominent in the parieto-occipital region. Unfortunately 1 of 5 patients died, and 3 of them became vegetative, follow-up MR images of whom showed brain atrophy and hyperintensity changes in the white matter (T2WI). Another patient who had minor changes on MR images recovered well. MR findings in patients with hypoxic brain damages corresponded well to the neuropathological findings described in the literature.

\section{SPACE 1685}

Evaluation of perfusional abnormalities in carotid occlusive disease by transcranial Doppler (TCD) and 99mTCHMPAO brain SPECT

M. G. Toni (Presenter), L. Fiori, G. Parenti, F.Marconi, C. de Gaudio, A. Carpi; Pisa

After carotid occlusion, collateral circulation inadequacy might cause cerebral hypoperfusion with chronical cellular impairment and increasing risk of ischemia after embolic event. To verify the compensatory system efficiency and select patients for surgery, in the last two years we evaluated 127 pts with carotid occlusion by doppler continuous wave, TCD and CT; 33 pts underwent $99 \mathrm{mTcHMPAO}$ brain SPECT as well. The pts were divided into two groups according to the presence (group 1) or not (group 2) of a CT stabilized ischemic lesion, and into subgroups according to their neurological symptoms. All pts were classified on the basis of TCD pulsatility index (PI) symmetry. Group 1: agreement between PI asymmetry and SPECT hypoperfusion in all cases but for a patient with stroke (PI asymmetry, SPECT hypoperfusional area corresponding to CT lesion). Group 2:6 pts with PI symmetry showed SPECT hypoperfusional areas ( 2 pts had bilateral occlusion justifying PI symmetry) and 4 pts had significant TCD mean velocity asymmetry on middle cerebral artery territory. SPECT can reveal, especially when CT is negative, areas of insufficient vascular flow not detectable using other techniques: TCD allows to identify the compensatory mechanisms and the haemodynamic situation to select patients for surgery.

\section{SPACE 1686}

MR imaging of sacral and perisacral lesions

Louis H. Wetzel (Presenter), Errol Levine, Solomon Batnitzky, A.W. Templeton; Kansas City, KS

Purpose: This exhibit demonstrates the utility of MR imaging in evaluating sacral and perisacral lesions.

Methods and Materials: Thirty-two lesions were studied using a superconducting 1.0-T MR imager. Eleven primary and 13 metastatic tumors, four congenital lesions, and four arachnoid cysts were evaluated.

Results: MR did not usually enable a more specific histologic diagnosis than other imaging techniques. However, extraosseous and proximal spinal extent of tumors was well defined without use of oral or intravenous contrast material. MR imaging is an excellent noninvasive technique for evaluating most sacral and perisacral lesions and is particularly helpful when precise tumor extent must be determined for treatment planning.

\section{SPACE 1687}

High-Dose Gadolinium-enhanced MR study in the evaluation of CNS metastases

William T.C. Yuh (Presenter), Jack D. Engelken, Michael G. Muhonen, Nina A. Mayr, David J. Fisher; lowa City, IA

We studied the efficacy of higher doses of gadolinium in the evaluation of CNS metastases. Twenty-three patients with a clinical suspicion of CNS metastases were prospectively studied with gadoteridol (ProHance ${ }^{\circledast}$, Squibb Diagnostics), a nonionic, low osmolality contrast agent. Each patient received an initial injection of $0.1 \mathrm{mmol} / \mathrm{kg}$ gadoteridol, followed 30 minutes later by an additional dose of $0.2 \mathrm{mmol} / \mathrm{kg}$. No clinically significant effects were noted. No significant difference in lesion contrast was noted between the images obtained immediately after the initial injection of gadoteridol and those obtained after 10 and 20 minutes. After the additional dose of gadoteridol. however, there was significant improvement in visualization $(70 / 71)$ and detection (42 new lesions in $18 / 23$ patients) of lesions. The initial-dose $(0.1 \mathrm{mmol} / \mathrm{kg})$ examinations identified 22 areas that were possible metastatic lesions. The high-dose $(0.3 \mathrm{mmol} / \mathrm{kg})$ examinations found 19 of these areas to contain definite lesions and one to contain a possible lesion and allowed the other two possible lesions to be excluded. Two patients had no detectable lesions. The additional information gained by high-dose examinations contributed to a potential modification in the treatment of $9 / 23$ patients.

\section{SE Head and neck radiology (08) SPACE 1688}

Assessment of solitary thyroid nodules using combined radiologic diagnostic methods

K. K. Altuğ (Presenter), S. Mercan, S. Özarmağan, A. Dinçağ; Istanbul

Various diagnostic methods have been used for the diagnosis of solitary nodules of the thyroid gland. In our study, we compared the histopathologic results in a series of patients having solitary thyroid nodules with the findings obtained from computerized axial tomography (CAT), scinti-scanning and needle aspiration biopsy. Scinti-scanning of the thyroid was done in all the patients. Ultrasound (US) demonstrated solitary nodules in 28 patients, cystic degenerated nodules in 16 patients and simple cysts in 3 patients. At the histopathologic examination, thyroid carcinomas were found in 7 patients who had solid solitary nodules and in $\mathbf{4}$ patients who had cystic degenerated nodules. CAT was done in 36 patients and demonstrated solid solitary nodules in 16 cases, cystic degenerated nodules in 17 cases and simple cysts in 3 cases. CAT has demonstrated more clear cut evidence of infiltration to surrounding tissues and cervical lymph nodes.

Needle aspiration biopsy was done in 33 cases. Accurate diagnosis was revealed in 31 cases, hence the significance of this method was $94 \%$. The rate of malignancy in cold solitary nodules was $22 \%$. US and needle aspiration biopsy obtained better results especially when they were combined with the scintiscanning method. Nevertheless it should be remembered that the role of CAT is 
very important in malignant cases and in cases having suspicious evidence of malignancy.

\section{SPACE $\underline{1689}$}

Uncommmon tumors of the parapharyngeal space, report of three cases J. Barcelo (Presenter), J. Teruel, J.C. Vilanova, J.M. Trull, A. Maroto. R. Bassaganyas, X. Serres; Girona

We describe three parapharyngeal space tumors whose origin does not belong to the typical bordering structures (parotid gland, pharynx, pterygoid muscles and the vascular bunch).

The first case appeared to be a minor salivary gland monomorphous adenoma. The second one was a squamous cell carcinoma of the epidermis, and the third one a cystic adenoid carcinoma or a minor salivary gland cilindroma. There was no defined border between the parotid gland and the mass in both cases of minor salivary gland tumor. In the other case there was a deep infiltration displacement of the pharynx fat to the inner, making impossible to separate from a parotid gland tumor.

Computed tomography (CT) offers a consistently good examination for the involvement of the parapharyngeal spaces, and contributes to predict the origin of the tumor according to the displacement pattern of the bordering fatty planes.

\section{SPACE 1690}

Nose and paranasal sinuse inflammatory disease: the performances of imaging techniques

M. G. Bonetti (Presenter), T.Scarabino, G. M. Giannatempo, A. Casillo, U. Salvolini, M. Cammisa; Roma

Inflammatory disease of nose and paranasal sinuses is a common challenge in diagnostic imaging. Conventional radiography $(\mathrm{RX})$ is mostly employed as a first step diagnosis. Alternatively a newly developed technique named computed radiography $(C R)$ can be used: photostimulable phosphor imaging plates are employed with the following advantages: exposure dose reduction, digital conversion of $x$-ray image information with image processing capabilities, wide exposure and registration range.

In this paper a comparison was made among $R X, C R, C T$ and $M R I$ in a series of 56 adult pts., using a craniostat (4 standard projections), a TCR 201 system, a high resolution CT $\left(\mathrm{OM}-10^{\circ}\right.$ plane, $8-10$ slices of $\left.10 \mathrm{~mm}\right)$ and a $0.22 \mathrm{~T}$ resistive magnet (multiplanar acquisition, 10-12 slices of $10 \mathrm{~mm}$, Tl-, PD- and T2-WI). The following aspects were considered: spatial resolution, dosimetry, time consumption, anatomical detail, costs and diagnostic outputs; a global performance index was derived $(\mathrm{RX}<1, \mathrm{CR}=1, \mathrm{MRI}=2.2, \mathrm{CT}=7.3)$.

$C T$ and, to a greater extent MRI, resulted superior to RX and CR; MRI is the most sensitive technique, while CT is to be proposed as the method of choice when looking also at cost analysis.

\section{SPACE 1691}

Exophthalmos produced by periorbital diseases with orbital involvement

M. Céspedes Más (Presenter), J.S. Martínez San Millán, A. Bernardo Fernández, J. Garcia Segovia, M. Muñoz Beltrán, C. Perdices; Madrid

We are presenting our experience in the study with $\mathrm{CT}$ of 20 patients with exophthalmos produced by orbital involvement by periorbital diseases during 18 months. There were 7 mucoceles ( 3 ethmoidal and 4 frontal), 1 aspergiliosis, 4 meningiomas, 2 osteomas ( 1 frontal and 1 ethmoidal), 1 osteosarcoma, 1 adenoid cystic carcinoma, 1 adenocarcinoma, 2 orbital cellulitis and 1 meningoencephalocele.

\section{SPACE 1692}

Vestibular aqueduct dilatation: a still underestimated cause of congenital deafness

N. Sellier, C. Maudelonde, M. Gandar, E. Coderc (Presenter); Bondy

a) Isolated vestibular aqueduct dilatation is a rare cause of fluctuant hearing loss and vertigo. We report 17 cases of such anomaly.

b) The diagnosis is based upon computed tomography on an axial view; the normal aqueduct has a shape of an inverted " $\mathrm{J}$ ".

In one case, MRI discloses the presence of lymphatic fluid in an enlarged vestibular aqueduct and endolymphatic sac.

c) According to the different segments of the vestibular aqueduct on $\mathrm{CT}$, one can differentiate several types of aqueduct dilatation:

- dilatation of the long limb of the " $\mathrm{J}$ " often unilateral ( 3 cases),

- dilatation of the short and long limbs of the "J" (5 cases),

- dilatation of the two limbs of the " $\mathrm{J}$ " with posterior petrous building corresponding to a large endolymphatic sac ( 9 cases).

These two last types are most often bilateral and the value of MRI must be pointed out.
SPACE 1693

Comparison of US, CT and MR in the evaluation of malignant uveal melanoma

O. Çokyüksel (Presenter), U.Korman, G. Öğüt, U.Denizci, A. Altuğ; Istanbul

Malignant uveal melanoma is the most common ocular neoplasm in adults. Those involving ciliary body and choroid are thought to originate from pre-existing nevi.

US, CT and MR findings of 9 cases with malignant uveal melanoma were compared. Following enucleation 8 cases were proved pathologically. One of the cases was presumed to be a small malignant melanoma who underwent radioactive implant treatment.

All three methods were accurate in determining the location of the tumor. Compared to the other modalities CT was especially effective in detecting the episcleral invasion and evaluating the morphology of the tumor.

Ultrasonographic B mode examination revealed typical findings of melanoma, and $A$ mode examination demonstrated the kappa angle which was strongly suggestive in the diagnosis of malignant uveal melanoma.

On MR the characteristic $\mathrm{T} 1$ and $\mathrm{T} 2$ shortening of the melanoma was thought to be the result of the paramagnetic effect of melanin.

\section{SPACE 1694}

Thyrold nodule: a comparative study of US versus PAAF and surgery B. Cortina (Presenter), N. Alegre; Madrid

Purpose: We make a retrospective study about the diagnostic reliability of the US in 160 patients which came to our Radiology Service with the diagnosis of thyroid nodule. We compare the US findings with those of punction-aspiration and both with surgical findings.

Methods and Materials: We make a statistical analysis comparing the sensitivity and specificity of all methods.

Results: Of the 160 cases, 143 was women and 15 men. It was made a citologic diagnosis of papilar carcinoma in 8 cases, folicular carcinoma in 1 case, folicular proliferation in 30 and proliferation of Hürtle's cells in 4 cases. We suggest the follow-up of patients with the last two diagnoses.

\section{SPACE 1695}

High resolution CT for the detection of cervical lymph node metastases Stefan Delorme (Presenter), Michael V. Knopp, Hans-Ulrich Kauczor, Friedhelm Raue, Heinz Buhr, Gerhard van Kaick; Heidelberg

Purpose: To exhibit the advantage of a CT technique with improved detail resolution in imaging of the cervical region.

Methods: 30 patients enrolled in a clinical follow-up-study who had undergone thyreoidectomy and modified neck dissection for medullary thyreoid carcinoma were studied in continuous slices with $4 \mathrm{~mm}$ thickness from the mandibula to the carina. The patients received intravenous non-ionic contrast medium at a controlled infusion rate. Images were reconstructed with a high resolution algorithm at an average $16 \mathrm{~cm}$ field of view. Attenuation values of vessels, lymph nodes, muscles and sonographically suspected metastases were measured and compared to previous studies without contrast medium and with normal technique. All patients were also studied by ultrasound, and concordance of sonographic and CT findings was assessed.

Results: The presented technique enables a significant improvement of contrast between muscular and vascular structures. The improved resolution as well as the optimized contrast enhancement by a controlled infusion technique enables excellent anatomical identification of all structures; lesions as small as $5 \mathrm{~mm}$ were reliably identified as adenopathy which could be confirmed by surgical resection. CT was superior to sonography in the deeper sternocleidomastoid region and the lateral cervical region. Sonography proved superior in the paratracheal region and in the jugular and medial retroclavicular region.

\section{SPACE 1696}

The effectiveness of Water's graphies in the interpretation of paranasal sinus infections: an analogy with magnetic resonance imaging Baris Diren, Oguz Dicle (Presenter), Dinc Ozaksoy, Tugrul Pirnar; Izmir

Water's graphy of the paranasal sinuses is one of the most popular conventional techniques used in the routine examinations of sinus pathologies. In recent years Magnetic Resonance Imaging (MRI) brought the most sensitive diagnostic possibilities and became a gold standard in studying the paranasal sinus abnormalities. In our study, we examined the effectiveness of Water's graphies in the detection of the acute and chronic sinus infections and had some conclusions about the conventional interpretation criterias.

With this purpose 100 patients, who were diagnosed to have sinus infections on MRI were subsequently examined with Water's graphies with standard techniques. These radiographs were interpreted by five different radiologists blindly and independently. The results were correlated with MR findings and 
analyzed with Receive Operating Characteristics (ROC).

Despite the high sensitivity of the Water's graphies, the accuracy in detection of the maxillary sinus infections was found as low as $56 \%$. The accuracy rate for frontal and ethmoidal sinuses were even lower.

\section{SPACE 1697}

No-tumoral pathology of the salivary glands: sonographic evaluation M. J. Ereño (Presenter), J. C. Ruiz, J. Negueruela, J. C. Martin, R. Mugica, O. Rodriguez; Bilbao

Purpose: Study of the efficacy of sonography for the diagnosis of no-tumoral diseases of the salivary glands.

Methods and Materials: Sonography of the parotid and/or submandibular glands was performed in 113 patients with clinical history of salivary pathology. The age of the patients ranged from 13 to 82 years. 46 were female and 67 were male.

Histology, follow-up or sialography in other cases were performed as the gold standard.

High frequency transducers ( 5 and $7.5 \mathrm{MHz}$ ) were used. The salivary glands on both sides were examined in transverse and longitudinal sections.

Results: Sonography has identified: 40 calculi (33 submandibular and 7 parotid), 15 parotid inflammations, 31 submandibular inflammations, 10 abscesses, 4 fistulas, 12 lymphadenopathy and 1 hypertrophic masseter. Conclusions: In acute inflammation sonography helps to define abscesses, fistulas or sialectasia, but it is worse to detect chronic inflammation. Sonography is also very useful in salivary calculi in most cases, especially in concrements larger than 2-3 mm and intraglandular location.

\section{SPACE 1698}

\section{High resolution CT: anatomy of the ethmoid}

J.C. Ferrie (Presenter), P.Vandermarcq, O.Azais, J.M. Klossek, C. Gasquet; Poitiers

Subject: The improvement of endoscopic endonasal surgery allows limited surgical resection in chronic rhinosinusitis. This functional sinus surgery needs an accurate topographic evaluation of diseases. The preoperative radiological investigations require a precise knowledge of ethmoid and ostiomeatal unit anatomy.

Methods: Thin section, axial and coronal plains, by high resolution CT were performed on 100 patients. CT correlation with dry skull specimen in same plains and endoscopic view in these patients were evaluated.

Results: Many morphological variations are found. However some landmarks are constant: the unciform process, the ethmoidal bulla, the middle and superior turbinate. All of them have ethmoidal bony roots. They form the base of ostiomeatal unit and ethmoid air cell system anatomy.

The different grooves for paranasal sinus draining and their relationship with the ethmoid are illustrated.

\section{SPACE 1699}

Middle ear pathology: correlation of $\mathrm{CT}$ and operative findings Z. Filaković (Presenter), Z. Maksimović, K. Glavina, O. Rubin, G. Šarić, D. Marjanović, B. Skvorcov; Osijek

Diseases of the middle ear and mastoid can be reliably diagnosed and described by CT. Our intention is to demonstrate the high potential of CT in preoperative diagnostics of patients suffering from middle ear pathology by comparison of CT and operative findings.

High resolution CT scans with $1 \mathrm{~mm}$ thick slices on SIEMENS Somatom DR were used to examine 22 adult patients. In 10 of them CT examination was followed by surgery. Clinical diagnoses ranged from suspected cholesteatoma to chronic middle ear inflammation with granulations and destruction of small ear bones.

Attic cholesteatoma visible on CT scans was found in 6 patients. The smallest one occupied only the epitympanical recessus while the largest one fulfilled the entire atticoantral space with tympanic cavity and propagated inflammatory process to the temporal cerebral lobe. Granulations were found in all cholesteatoma patients and majority of them had small ear bones destructed. One patient had isolated anvil destruction without cholesteatoma.

Surgery confirmed CT visible pathology (granulations, liquid content, adhesions) in operated patients. Surgery planning was influenced by $C T$ images in some patients, particularly in choosing appropriate approach or in prepairing reconstructive surgery for hearing improvement.
SPACE 1700

Radioiodine therapy of a struma with tracheal stenosis: can a CT study make a contribution to the documentation of the result of the therapy? Jürgen Frohn (Presenter), Volkmar Jacobi, Detlev Rübesam, Gustav Hör; Frankfurt am Main

For patients with a struma and with symptoms of a mechanical displacement of the trachea, radioiodine therapy is generally suggested as an alternative therapy, if the risk of surgery is increased, and if the iodine uptake is sufficiently high. Investigations of the struma reduction therapy with Iodine-131 have shown that an objective assessment of the result of the therapy is difficult. The goal of our study was to determine whether CT studies of these patients can provide additional information for an objective assessment of the result of the therapy.

A CT study is able to image the intraluminal and extraluminal structures of the trachea non-invasively and without administration of a contrast medium. Comparability of CT measurements prior to and after radioiodine therapy is, however, impaired by the individual selection of the slices in the scout view. Even investigators with substantial expertise experience deviations of up to several millimeters when trying to reproduce the same slice. Such shifts lead to significant differences in the measurement of the tracheal lumen. For the quantification of the result of the therapy the CT study has, therefore, only limited usefulness.

As a complement to the measurement of the circumferential of the neck, the determination of the thyroidal volume, and lung function studies, the CT study of the trachea can make a contribution to the documentation of the result of the therapy. It can, however, not provide any information which would be additional to a conventional radiograph of the trachea.

\section{SPACE 1701}

Ultrasonographic volumetry of the thyroid in school children related to the iodine intake

Judit Gönczi (Presenter), Klara Sajgó, lldikó Farkas; Budapest

The purpose of the study was to survey the goiter prevalence in school-children from classes 1,5 and 7 in different areas of Hungary. The capital served as control, the other towns were chosen because of the high goiter incidence, the decreased iodine supplementation. Ultrasonographic volume determinations were correlated to the age and the body weights and compared to the estimated volume results by palpation and inspection. The ultrasonographically measured thyroid volume corresponds to the iodine intake and the urinary iodine excretion. This measurement may prevent the overestimation of goitre prevalence in epidemiological surveys. The effectiveness of prophylaxis will be measured by goitre frequency data after five years.

\section{SPACE 1702}

PET in patients with advanced head and neck cancer: measurement of early metabolic changes during combined therapy

Uwe Haberkorn (Presenter), Ludwig Strauss, Antonia Dimitrakopoulou, Michael Knopp, Eberhard Seiffert, Christoph ReiBer, Wolfgang MaierBorst; Heidelberg

PET using fluorodeoxyglucose (FDG) delivers quantitative data about the glucose uptake and can be used to study therapy induced changes in the tumor metabolism. In 12 patients PET studies were performed prior and after the first cycle with cisplatinum and 5-FU. Tumor and lymph node volumes were determined in CT cross sections and the growth rate was calculated. The images were analysed using a region of interest technique and the data were standardized to the injected dose and the body weight. FDG data were evaluable for 6 tumors and 8 lymph nodes, volumetric data for 5 tumors and 7 lymph nodes. All malignant lesions showed an increased FDG uptake as compared to normal muscle tissue. During therapy $50 \%$ had a decrease in FDG uptake, the rest remained unchanged or increased. Multiple lymph nodes in the same patient may show a different baseline value and also a difference in response to therapy. A high correlation between the change in FDG uptake and the growth rate was found with different regression functions for tumors and lymph nodes. These results demonstrate that early changes in tumor uptake due to therapy can be detected with PET.

\section{SPACE 1703}

Narrow beam radiography and spiral tomography in evaluation of bone grafting in the mandible

D. Hallikainen (Presenter), A.-L. Söderholm, C. Lindquist, P. Paukku, J. Edgren; Helsinki

Purpose: Radiological evaluation of integration and complications of bone grafting in the mandible.

The material consists of 21 patients subjected to mandibular resection, reconstruction and bone grafting. 
Radiological examinations included panoramic views, detailed images with narrow beam radiography and cross-sectional tomography.

Methods: The radiographs were evaluated for bone resorption within the graft and under the plate, changes in graft size, integrity of reconstruction plate and bony union. The findings were recorded during follow-up and compared to clinical signs.

Results: Bone resorption is an early sign of graft failure, and may occur before clinical signs. Resorption is first visible under the plate. A broken reconstruction plate indicates always failure of bony union.

Detailed narrow beam images and cross-sectional tomography are effective in bone graft evaluation.

\section{SPACE 1704}

Transtemporal catheterization of the lingual artery for intraarterial chemotherapy

A. Hlava (Presenter), A. Krajina, A.Šimủnek, E. Hozová, Z. Jirousek; Hradeć Králové

The authors present a technique of long-term selective lingual artery catheterization for intraarterial regional chemotherapy. The transtemporal approach enables safe introduction of catheters through the superficial temporal artery. Catheterization technique is presented in 19 patients with the diagnosis of carcinoma baseos oris and carcinoma linguae. Both the lingual arteries were infused simultaneously in four patients with the tumors spreading over the midline. The dislocation of the catheter was found for eight times of twentythree catheterizations. The following catheter reposition was always successful.

\section{SPACE 1705}

\section{Two cases of enlargement of the M. Masseter}

M. Horvath (Presenter), J. L. Termote, S. F. Lemahieu; Leuven

We want to present two patients with a swelling in the mandibular region on the left side. The clinical course in the two cases was nearly identical: a painful, rapidly growing mass lesion of the left cheek without temperature nor history of trauma.

A scan was performed and again the CT appearance was identical in the two cases: the m. masseter was inhomogeneously enlarged with areas of liquid density and only slight infiltration of the subcutaneous fat. There were no signs of bone involvement.

A biopsy was performed and revealed non-hodgkin lymphoma in one case and myositis in the other case.

Concluding that lymphomatous and inflammatory involvement of the $\mathrm{m}$. masseter cannot be differentiated on imaging grounds.

\section{SPACE 1706}

\section{MR imaging of supraglottic cancer}

M. Horvath (Presenter), J. L. Termote, S. F. Lemahieu; Leuven

We wanted to establish the value of MR in the diagnostic work-up of supraglottic cancer. Retrospectively a study was made of 15 patients with supraglottic carcinoma. CT and MR of these patients were carefully compared with respect to tumor delineation. MR was superior in 7 cases, MR and CT did equally well in 7 cases and in I case MR was inferior (due to motion artifacts). MR was able to delineate the fibrocartilage of the epiglottis, with respect to the tumor. MR showed to a better extent the extension into the base of the tongue and the pre-epiglottic space. Moreover MR was a valuable tool for the follow-up of carcinomata treated with radiotherapy.

Concluding that to us MR is the method of choice for the diagnosis and follow-up of supraglottic cancer.

\section{SPACE 1707}

\section{The technique and advantages of digital subtraction sialography} M. O. Cizmeli, E. T. Ilgit (Presenter); Ankara

Sialography is a useful investigation in the diagnosis and management of inflammatory and calculous diseases of the parotid and submandibular salivarys glands. Its use for space-occupying lesions of the region is controversial. High contrast between bone and air in the sinuses and pharynx and overlapping structures in the head and neck region impede clear demonstration of the ducts of salivary glands in conventional sialography. Digital Subtraction Sialography (DSS) is an important non-vascular application of digital radiography. High-contrast resolution and advantage of subtraction improves quality in digital imaging of salivary glands. In 62 patients with suspected inflammatory, calculous disease or space occupying lesions, we performed 50 parotid and 16 submandibular DSS. Following the insertion of catheter, $0.5-1 \mathrm{ml}$ lipiodol was injected after the acquisition of mask images. Image acquisition rate was $1 \mathrm{fr} / \mathrm{sec}$. for 10 seconds and pulse width was $50 \mathrm{msec}$. The images were stored in a $512 \times 512 \times 10$ bit memory. Image selection and subtraction, reregistrations, window manipulations and other post-processing facilities were performed. Although the value of sialography has been debated, we think that in comparison to conventional sialography, DSS is a less time-consuming examination with less radiation exposure and superior image quality.

\section{SPACE 1708}

\section{Evaluation of thyroid nodules by $\mathrm{CT}$ value}

Y. Imanishi (Presenter), N. Ehara, K. Sakuyama, T. Itoh, T. Ishikawa, T. Shinagawa, T. Yamaguchi, K. Kuroki; Kawasaki

To make a diagnosis of thyroid nodules based on their iodine concentration as measured by CT, thyroidectomy was conducted on 50 thyroid nodules ( 29 benign nodules, 21 malignant nodules) of 35 patients ( 32 females, 3 males). Each nodule was pathologically diagnosed. These patients had undergone CT examination of the thyroid without intravenous contrast material before surgery. lodine concentration in fragments $(5-120 \mathrm{mg})$ of operative thyroid tissues was measured by neutron activation analysis. CT scans were obtained using contiguous $5-\mathrm{mm}$ collimated sections through the thyroid without intravenous contrast material. Correlation between C.T value and iodine concentration in thyroid tissues was evaluated. Histograms of CT values of the nodule in all images were presented by $\mathrm{CT}$ computer from the region of interest (ROI) along the margin of the nodule. Correlation between iodine concentration and CT value was most nearly expressed as follows: $(n=31), r=0.96)$; Iodine Concentration $=(\mathrm{CT}$ value -65$) / 104(\mathrm{mg} / \mathrm{g})$.

Using this formula, twenty-one (75\%) of 28 adenomas had a CT value of less than 65 , showing little iodine concentration, whereas all 15 carcinomas had, at least partially, a C.T value of more than 65 . Adenomas with partial malignancy showed an intermediate pattern of CT value distribution.

\section{SPACE 1709}

\section{Encephalocoele of the petrous bone} Angel Mironov (Presenter); Aarau

The rare occurrence of an encephalocoele through a defect in the tegmen of the petrous bone is reported. A male, aged 43 years, was referred for investigation following two episodes of meningitis. Neurology examination showed the patient to be lethargic.

CT scan of the middle ear showed a soft tissue opacity in the right atticoantral area with evidence of a deficiency of the related tegmen. The other areas of the middle ear and mastoid air cell system suggested a chronic infection or a cholesteatoma.

An MRI was performed, confirming the area of dehiscence and suggesting the presence of a small encephalocoele with a signal intensity similar to brain within the cavity. There was an abnormal inflammatory signal intensity in the adjacent mastoid air cells. On surgical exploration a soft pulsating brain mass was noted in the atticoantral region, covered with granulation tissue and thick dura mater. The intraoperative conclusion was that this was an encephalocoele occupying the atticoantral region, having prolapsed through a secondary tegmen dehiscence following a cholesteatoma.

The case illustrates the association of this abnormality with recurrent meningitis and the importance of imaging in defining the nature and site of the lesion prior to surgery.

\section{SPACE 1710}

Nasopharyngeal carcinoma with skull base and intracranial involvement: correlation MRI (plain \& enhanced) and CT with bone target images

T. Miura (Presenter), N. Hirabuki, T. Hashimoto, M. Mitomo, K. Harada, R. Kawai, T. Kozuka; Osaka

Purpose: To describe the experience with Gd-DTPA MRI and CT with bone target images of the nasopharyngeal carcinoma with skull base and/or intracranial involvement.

Methods: To date, ten patients suffering from primary and recurrent nasopharyngeal carcinoma with skull base and intracranial involvement were examined with plain \& Gd-DTPA-enhanced $T 1$-weighted $(T R / T E=420 / 15) \&$ plain T2-weighted sequences $(T R / T E=2000 / 90)$ in transverse \& coronal sections. CTs are also examined by the same sections with bone target images. Results: Gd-DTPA MRI can demonstrate tumor extension within the soft tissue clearly. Subtle cortical bony changes can be better demonstrated by bone target CT images.

Conclusions: Gd-DTPA improves the diagnostic abilities about the extension of nasopharyngeal carcinoma within the soft tissue. Subtle cortical bony change can be diagnosed better by bone target CT. 


\section{SPACE 1711}

Three dimensional computed tomographic reformation of head Akiko Ohno (Presenter), Minoru Ueda, Kazuko Ohno; Nagoya

Purpose: In our hospital, three dimensional (3D) computed tomographic reformations have been used as an adjunct to standard Axial computed tomography $(\mathrm{CT})$. They were carried on with the purpose of precisely evaluating congenital abnormalities of intracranial tumor, concaved or disordered face caused by traffic accident and detection of recurrence tumor. Some of them were color displayed.

Methods: Our clinical studies were performed on a Siemens DPH scanner and reconstruction program 3DCT (software), $420 \mathrm{mAs}$ were employed with $120 \mathrm{kVp}$. Each scan time was within 4 seconds on condition with narrow section of 1-2 mm. For each case was used 30 scans. About 500 cases were examined with best image quality.

Results: The complexes feature of bony structure were well demonstrated because of reduction of motion and noise artifact. This is due to rapid scanner with well qualified software.

\section{SPACE 1712}

Ultrasonography of the larynx and the vocal cords

Orhan Oyar (Presenter), Erkan Sevinc, Nevra Elmas, Hadi Özer; Izmir

It is seen that the recent sonographic studies directed towards the evaluation of the larynx, tongue and mouth floor promise great progress in the future. Especially the real time imaging of the vocal cord movements is the main advantage of US. In this study, the normal and pathologic laryngeal cases are examined with a $7.5 \mathrm{MHz}$. linear, water pad transducer. The vocal cords are tried to be imaged both stationary and during fonation.

The thyroid cartilage is seen as an inversed $U$ or $V$ shaped uninterrupted echogenity in the sections obtained by transverse projections. The false cords have echogenic and the true vocal cords have hypoechogenic internal structures and they are both seen as symmetrical, triangular formations which show pendular movements during fonation. The vocal cord paralysis is detected by the loss of the typical movements and the destruction of the thyroid cartilage is explained by the interruption of the echogenity. In addition to this, especially the larynx carcinomas localised in the supraglottic region, the preepiglottic invasion of the tumor and the attraction of the enviromental lymph nodes can be determined sonographically.

Sonography, together with the laryngoscopic methods is applied successfully in the evaluation of the larynx and vocal cords.

\section{SPACE 1713}

Videofluoroscopy of free jejunal graft reconstruction for vocal rehabilitation

Peter Pokieser (Presenter), Wolfgang Schima, Konrad Uranitsch, Matthäus Grasl, Thomas Helbich, Klaus Ehrenberger, Dimiter Tscholakoff; Vienna

Purpose of our study was to evaluate the protective function of free jejunal grafts (Speech Siphons) in laryngectomized patients against aspiration.

23 examinations were performed in 14 patients ( 2 patients presented clinically with aspiration), 12 patients without aspiration using videofluoroscopy (50 frames/second) and high density barium. Following parameters were evaluated

1) Boluspenetration (BP) from the hypopharynx into the graft:

a) no penetration b) penetration limited to the ascending limb of the Speech Siphon c) penetration limited to descending limb d) penetration into the trachea

2) The minimal distance from the level of the hypopharyngeal anastomosis to the vertex of the Siphon.

Results: 1) 10 patients presented boluspenetration limited to the ascending limb of the Siphon. One had no boluspenetration; I asymptomatic patient and both patients with clinical aspiration had a boluspenetration into the descending limb.

2) The minimal distance was reduced to zero in patients with aspiration as opposed to the patients without aspiration (distance: $20-50 \mathrm{~mm}$ ).

Conclusions: Boluspenetration into the ascending limb is not a pathologic finding. The vertex of the Siphon should not reach the level of the hypopharyngeal anastomosis during swallowing, if so aspiration may occur.

\section{SPACE 1714}

CT and ultrasound evaluation of cervical metastatic lymphadenopathy N. Raffetto (Presenter), M. Trinci, A. Vitturini, E. Banelli, V. Donato, C. Trinci; Roma

In 60 patients with head and neck tumors, lymph nodes involvement was assessed by physical examination, CT and ultrasonography.

Positive lymph nodes were detected with palpation in 26 patients $(44 \%)$ and by CT and ultrasound scans in $34(56 \%)$. Forty positive nodes were identified with physical examination, 61 by CT and 70 by ultrasonography.

CT and ultrasound can visualize deep metastatic nodes in the neck, difficult to discover with palpation, more over this cannot evaluate whether or not the jugular vein is truly thrombosed. Ultrasonography allows to distinguish a single enlargement in nodal masses and gives an accurate evaluation of the relations between nodes and vascular structures.

CT is of unquestionable value in the study of retropharyngeal nodes, moreover permits to determine the number and position of enlarged nodes and their relation with the neck vessels.

\section{SPACE 1715}

\section{Sialography, usefulness and images}

A. Rosendo González (Presenter), M. D. Martorano Navas, J. C. Albillos Merino; Madrid

There are many methods for the radiologic examination of the salivary glands: plain file radiography, conventional sialography, computed tomography, ultrasound, radioscintigraphy and magnetic resonance.

A summary of the indications and contraindications of conventional sialography is shown in this poster, and also its usefulness in the diagnosis of the different pathologic conditions which involve the salivary glands: calculi and calcifications: inflammatory disorders and infections and tumors and cysts. We conclude, on the basis of our experience and the bibliographic review that, while computed tomography is the method of choice for the examination of mass lesions and bland enlargement of one or several glands, sialography is the optimal method to demonstrate calculi, strictures, sialectasias and fistulas.

\section{SPACE 1716}

Magnetic resonance Imaging of the nasopharynx and the skull base: normal anatomy

Matthias Schütz (Presenter), J. Gaa, H. K. Deininger; Darmstadt

Purpose: Skull base, nasopharynx and related spaces are crossover areas between the extra- and intracranial structures of the head and neck. They are pathways of tumors and inflammatory diseases. We analyzed the normal anatomy of the skull base with magnetic resonance (MR) imaging using for a teaching model.

Methods and Materials: Head and neck from three healthy volunteers were studied by 1,5 T MR imaging. Tl-weighted images (repetition time $=500 \mathrm{~ms}$. and echo time $=15 \mathrm{~ms}$.) were obtained in axial, coronal and sagittal plains using $5 \mathrm{~mm}$ slice thickness. The axial MR images were correlated with wholeorgan sections from a cadaver and with computed tomography (CT).

Results: Parapharyngeal space, infratemporal fossa, pterygopalatine fossa, the skull base with its foramina, the cranial nerves and superficial soft tissues are well seen and demonstrated in MR images. The inherent contrast between fat, muscle and bones allows excellent visualisation of these anatomic details. Conclusions: MR imaging gives a detailed anatomic view of head and neck. It is an excellent possibility for learning anatomy in different planes.

\section{SPACE 1717}

Evaluation of scintigraphy of the salivary gland by factor analysis in patients recelving radiation therapy for the head and neck Yoshihiro Takeda (Presenter), Keiko Okuno, Tomio Nakagawa, Mitsuharu-Shimizu, Kōtaro Yasui, Yoshio Hiraki; Okayama City

Purpose: Evaluation was made of dysfunction of the salivary gland by factor analysis in patients receiving radiation therapy for the head and neck.

Methods and Materials: A total of 18 patients who were to receive radiation therapy for the head and neck were taken as subjects, and scintigraphy of the salivary gland was performed on them with ${ }^{99} \mathrm{mcO}_{4}$ before start and after completion of the radiation therapy. Then, study was made by 2 -factor analysis based on two factors - one being physiological component consisting of background such as oral excretion, and the other being salivary gland component. Comparisons were made between values observed before and after radiation therapy of the contribution rate of the salivary gland component and inclination formed by bringing the functional curve of the salivary gland factor into a linear approximation.

Results: The contribution rate increased from $26.9 \%$ to $34.8 \%$ showing a significant difference, while the linear inclination decreased from 2.18 to 1.57 also showing a significant difference. Factor analysis makes it possible to objectively assess the accumulation of ${ }^{99 \mathrm{~m}} \mathrm{TcO}_{4}$. Thus, it is useful in evaluation of the functioning of the salivary gland before and after radiation therapy for the head and neck. 


\section{SPACE 1718}

Sagittal autotomography on nasopharyngeal cavity to detect tumours Tan Tjin Joe (Presenter); Medan

There are many nasopharyngeal tumours in Indonesia. For 27 years (1961-1987) 8250 patients with malignant tumours were treated in St. Elisabeth Hospital. Out of these unselected patients, we found as the biggest group to be nasopharyngeal tumours $( \pm 19 \%)$ and lung tumours were rare $( \pm 2 \%)$.

The diagnosis was made by biopsy only and it wasn't possible to detect tumours neither with conventional tomography nor with CT scanner.

An experiment has been carried out using AUTOTOMOGRAPHY, an old principle described by Vallebona in 1930 entitled TRATATO DI STRATIGRAFIA, which employed the rotational movement of the patient, but utilized both a fixed X-ray tube and film.

My experiment has been performed on a fibreglass chair placed on a rotating disc with a fixed verticle axis. The chair can be moved to both sides to place the lesion on the axis. The chair can also be moved in the direction of the X-ray beam for making the different slices of the tomograms. The whole unit can be placed between the table and screen of an ordinary $X$-ray unit in upright position.

It has been proven that with this small and simple unit, tomograms of all parts of the human body can be made, also sagittal tomograms of the nasopharyngeal cavity to detect tumours and destructions of the base of skull.

\section{SPACE 1719}

The role of $\mathrm{CT}$ in clarifying the etiology of chronic sinusitis E. Tanács (Presenter), T. Hartai; Szeged

We carried out the CT examination of the paranasal sinuses of 25 patients with symptoms of chronic sinusitis. Clarifying the reason of the chronic inflammation we studied the so-called "osteomeatal unit" the role of congenital malformations and the after-effects of earlier operations. Patients were examined at the same time by endoscopy according to the same aspects. Results of the two methods were compared to decide the additional value of CT. Results of findings found during operation were compared to the results of the two above-mentioned diagnostic methods. Our experiences suggest that CT is an essential method in the surgical indication of chronic sinusitis.

\section{SPACE 1720}

Possible errors in positioning the skull using axial projections in the teleradiography: cephalometric evaluations

P. Terrosi Vagnoli (Presenter), M. A. Centra, P.Sacco, R. Volpe, R. Giorgetti, P. Nannelli, M. Mastripieri, F. Cerboni; Siena

The Authors purpose was reproduce, by positioning a mannequin's skull on a cephalostat, the most common errors which the radiologist makes taking axial teleradiogram of the skull.

The skull's movements were so classified: a) movements on the x-plane which represent the hypo- or hyperextension of the skull; b) movements on the z-plane representing right or left inclination.

The variations on the two-planes were demonstrated by taking axial teleradiograms. The cephalometric results were evaluated by $\mathbf{M}$. Langlade method for the axial projection.

Contrary to what other Authors affirmed, results obtain from this work clearly demonstrated that the wrong positioning of subjects undergoing cephalometric radiogram can cause remarkable variations in this diagnosis and suggest this choice of therapy not satisfactory the need of the patients.

\section{SPACE 1721}

Neck ultrasonography (US) versus total body scan (TBS) in the follow-up of patients with differentiated thyroid cancer (DTC) G. Tomasella (Presenter), D. Casara, D. Rubello, A. Piotto, M. R. Pelizzo, P. Boccato, M. Piccolo, B. Busnardo; Padova

In recent yrs an increasing interest on neck US and US guided FNA cytology in patients with DTC has been reported. The present study was undertaken to investigate the effective role of US in the follow-up of these pts, comparing US to TBS results. We considered 65 pts previously treated by total thyroidectomy for DTC and with elevated serum Thyroglobulin $(\mathrm{Tg})$ levels $(\mathrm{Tg} 3 \mathrm{ng} / \mathrm{ml}$ is considered pathologic in our laboratory for these pts). To search metastases (mts), they were studied by means of US, TBS, conventional radiology, CT scan and, in cases with lymph node (LN) mts, malignancy was proven by FNA cytology and/or histology. In 19 of them LN mts were documented, 11 presented mediastinic mts, 17 lung and 14 bone mts; in other 4 cases normal thyroid remnants were found. US-LN image was considered pathologic when the ratio of trasversal to longitudinal diameters was 0.65 and when $L N$ showed a hypo- or iso-echoic pattern. TBS resulted positive in 49 pts: $9 / 14$ (64\%) of bone $\mathrm{mts}, 9 / 11(82 \%)$ of mediastinic $\mathrm{mts}, 14 / 17(82 \%)$ of lung $\mathrm{mts}, 13 / 19(68 \%)$ of LN mts and in the 4 cases with thyroid remnants US revealed pathological images in $14 / 19(73 \%)$ of $\mathrm{LN}$ mts, and 3 of them were TBS negative. In pts with distant mts neck US resulted negative. It can be concluded that neck US investigation represents a useful, easy and sensitive technique in the follow-up of pts with DTC to search LN mts. It may be recommended to routinely associate neck US to $\mathrm{Tg}$ assay in the follow-up of DTC-pts. Moreover, in cases with elevated Tg levels and with negative neck US, TBS has to be performed to search distant $\mathrm{mts}$.

\section{SPACE 1722}

Three-dimensional $\mathrm{CT}$ in the head and neck diseases with bony abnormalities

Noriaki Tomura (Presenter), Masaaki Shindo, Takaharu Miyauchi, Yasuo Seino, Hideto Okada, Osamu Watanabe, Toshio Kato; Akita City

Purpose: To present our experiences with three-dimensional computed tomographic (3D-CT) technique in the head and neck diseases with bony abnormalities, and evaluate its value.

Methods: The subjects were 32 patients with various bony abnormalities, who consisted of maxillary sinus carcinomas, nasopharyngeal carcinomas, tumors in the skull base, fibrous dysplasias, skull fractures, craniosynostoses, and so on. A General Electric Model 9800 CT scanner was programmed to perform contiguous 1.5-mm serial sections. The 3 D83 General Electric Medical Systems software package was utilized to create the 3-D images. We reconstructed the images at the degree of rotation and angulation that best demonstrated the pathological lesions using a Data General Eclipse MV $/ 7800 \mathrm{C}$ minicomputer. Results: In 26 of 31 patients (84\%), 3D-CT clearly delineated the destructive or other abnormalities of bony structures, and was very helpful in planning therapy, especially surgical intervention. In the remaining 5 patients with a subtle lesion, 3D-CT did not demonstrate the lesions; for example, a minor blow-out fracture, nasopharyngeal carcinoma with a subtle erosion of the skull base.

Conclusions: 3D-CT was a useful technique in the diagnostic evaluation and planning therapy in the head and neck diseases with bony abnormalities.

\section{SPACE 1723}

Detection of temporo-mandibular joint (TMJ) dysfunction by SPECT bone scintigraphy: our experience

M. G. Toni (Presenter), A. Calderazzi, C. de Gaudio, L. Battolla, G. Testi, E. Cerretti; Pisa

Symptomatic TMJ dysfunction may affect about $25 \%$ of the adult population with a smaller, but significant percentage of patients suffering serious disability. From 1986 to 1989102 pts with significant TMJ symptoms and with various TMJ diseases, were evaluated with conventional radiology and closedopen-mouth TMJ tomograms; $20 \mathrm{pts}$ were submitted to CT and 5 to MR as well. SPECT and planar imaging were performed in 27 pts with a rotating gamma-camera equipped with a general purpose collimator. Transaxial, coronal, sagittal tomograms were reconstructed. TMJ increased radiotracer localization was regarded as a positive finding, and the intensity of TMJ activity was compared with that of adjacent calvarium using ROIs. Our results indicate that SPECT rather than planar imaging provides useful information for diagnostically difficult, symptomatic pts; CT although useful in assessing the TMJ structure and articular surfaces, is not always a good indicator of dysfunction: MR gives a more detailed image of soft tissue anatomy and may identity functionally significant TMJ abnormalities, but cost limits its application. Either MR or SPECT is suitable for non-invasive confirmation of the TMJ derangement clinical diagnosis, and if better joint diagnostic accuracy is desired both techniques should be employed.

\section{SPACE 1724}

Comparison between malignant lymphoma and anaplastic carcinoma of the thyrold using ${ }^{67} \mathrm{Ga}$ imaging, US and CT

K. Toshima (Presenter), K. Shimizu, A. Kubo, H. Nagakura, K. Ido, K. Ito, T. Shoji; Tokyo

Purpose: Malignant lymphoma and anaplastic carcinoma of the thyroid patients usually have a large goiter which enlarges rapidly in a short period of time. It is difficult to discriminate between them using typical clinical findings. In this report, we studied the differential diagnosis between malignant lymphoma and anaplastic carcinoma of the thyroid, using ${ }^{67} \mathrm{Ga}$ imaging $\left({ }^{67} \mathrm{Ga}\right)$, ultrasonography (US) and computed tomography (CT).

Methods and Materials: We studied 35 patients with malignant lymphoma and 14 patients with anaplastic carcinoma. Diagnosis was made by histological examination. ${ }^{67} \mathrm{Ga}$, US and CT were performed on all patients previous to treatment and the findings were analyzed using various factors.

Results: $1 .{ }^{67} \mathrm{Ga}$ : All cases except one, malignant lymphoma, had positive uptake. The uptake in malignant lymphoma tended to be stronger than that in anaplastic carcinoma. 2. US: Both tumors were demonstrated to be hypoechoic masses, but anaplastic carcinoma tended to be heterogeneous in comparison with malignant lymphoma. 3. CT: Malignant lymphoma was demonstrated to 
be expansive, while anaplastic carcinoma was demonstrated to be invasive. All cases of anaplastic carcinoma had calcification, while $4 \%$ of malignant lymphoma had calcification.

Conclusions: ${ }^{67} \mathrm{Ga}$ was more useful than US and $\mathrm{CT}$ in discriminating between malignant lymphoma, anaplastic carcinoma and other thyroid tumors. US and CT were more useful in appreciating the extension of tumor and clinical stage.

\section{SE \\ Pediatric radiology (09) SPACE 1725}

\section{Extravascular interventional radiology in pediatric urology}

C. Defilippi (Presenter), G. Di Rosa, G. Masi; Torino

Interventional radiology is routinary in adults but has only recently been introduced in pediatrics and in this age group its common application is in urological pathology. Since 1984,78 procedures were performed in the Radiology Department of our Hospital using the percutaneous route with echographic and or radioscopic guidance.

Percutanous nephrostomy: 62 cases.

Positioning of uretheral endoprosthesis: 1 case.

Drainage of renal abscess: 1 case.

Drainage of urinoma: 5 cases.

Evacuation of renal cyst: 4 cases (one with alcoolisation too).

Dilatation, using Gruntzig catheter, of urinary stenosis: 5 cases.

Patients were aged 24 hours-14 years. The interventions on newborns were performed under general anesthesia and the remaining cases under local anesthesia after sedation with chlorpromazine i.m. $(1 \mathrm{mg} / \mathrm{kg})$ when required necessary.

In 74 cases $(94,8 \%)$ the procedure was correctly performed while in 4 cases $(5,2 \%)$ this did not possible succeed: in 3 patients it wasn't possible to place the nephrostomic drain: in one patient the percutaneous dilatation of a stenotic uretherosigmoidostomy was functionally insufficient. Only in 2 cases $(2,5 \%)$ there were complications (endopyelic bleeding during percutaneous nephrostomy) that resolved spontaneously.

\section{SPACE 1726}

Low dose computed radiography of scoliosis in children: review of 1600 examinations

J. A. Kalmar (Presenter), C. B. Merritt, M. S. Kogutt, F. H. Warren, J.P. Jones; New Orleans, LA

Computed radiography using photostimulable phosphor receptors allows dissociation of $X$-ray dose from film density. This permits diagnostic quality radiographs of the thoracolumbar spine to be obtained with doses as small als 5 to $10 \%$ of those required using conventional film screen techniques. When used for serial evaluation, this technique has the potential for significantly reducing the cumulative radiation exposure in young patients followed for observation and treatment of scoliosis.

Over 1600 spine examinations in children ranging in age from 4 to 18 years have been performed using low dose techniques with computed radiography over a 5 year period. Records were reviewed to calculate the total savings in radiation burden resulting from the use of low dose techniques and the adequacy of diagnostic image quality in the serial evaluation of scoliosis. Our studies indicate that clinically useful studies can be routinely achieved at average exposure levels of less than $5 \%$ of those required for normal radiography, with significant reduction in cumulative dose to bone marrow and breast tissue in patients receiving serial examinations. Low dose computed radiography is recommended as the method of choice for serial evaluation of scoliosis in pediatric patients.

\section{SPACE 1727}

Strip scanning and averaging: a method to improve pediatric cardiovascular ECG-gated MRI

B. Kastler (Presenter), Ph. Germain, A. Livolsi, A. Gangi, R. Allal, F. Bonjan, M. Jeung; Strasbourg

Purpose: To improve image quality and shorten scan time in low aged pediatric patients with complex congenital heart disease (CHD).

Methods: We developed on our GE MR-MAX .5T imager a method using $5 \mathrm{~mm}$ slices with asymmetric matrices (M) and field of view (FOV) covering $30 \times 11.2 \mathrm{~cm}$ (adjusted to fit thorax of newhorns). We use of $256^{\mathrm{F}} \times 96^{\mathrm{P}}$ matrix with $1.2 \mathrm{~mm}$ large isotropic pixel reducing image time by $63 \%$ (and $\mathrm{S} / \mathrm{N}$ by $39 \%$ ). This allows routine use of 4 (or respect. 8) average which on the counterpart improves $\mathrm{S} / \mathrm{N}$ by $100 \%$ (respect. $182 \%$ ) and proportionally reduces signal of cardiac motion ghost artifact. Imaging time for one set of 5 images (with TR's of about $300 \mathrm{~ms}$ ) is around $2(\mathrm{~N}=4 \mathrm{Ex}$.) to $4 \mathrm{~min}$. $(\mathrm{n}=8 \mathrm{Ex}$.)

Results: This method has been evaluated in 30 newborns with various CHD.
Imaging procedure is better tolerated (20-30 min. for 4 to 6 runs). Moreover, improved image quality allows precise diagnosis of complex cardiac anomalies and demonstrates very small vascular structures like coronary arteries, pulmonary veins and palliative shunts.

Conclusions: MRI with strip scanning and averaging is a very rapid and efficient method for the evaluation of CHD in newborns.

\section{SPACE 1728}

Ultrasound examination of legg-perthes-calve disease

Hervé Leclet (Presenter), Pierre-Marie Delforge, Christian Morin; Berck sur Mer

Ultrasonography is not a common method to study LEGG-PERTHESCALVE disease (L.P.C.). However it seems to be useful method to appreciate femoral head deformity and to study articular cartilage. Twenty-one patients with LPC were examined prospectively. Ultrasound was compared to anteroposterior and lateral X-Rays and/or arthrography-Femoral head deformities and lateral articular cartilage are well defined. Thickness of femoral cartilage is variable and its echogeneticity is increased. Sub-chondral fracture is visible in the early stage of the disease. We have not observed hip joint effusion during the evolution. Ultrasound cannot appreciate acetabular deformity and thickness of the acetabular cartilage. Metaphyseal cysts and changes in shape of the femoral neck are not visible.

We conclude that ultrasonography can perhaps be a useful method to survey the evolution of cartilaginous femoral head deformity but it must not replace arthrography in the preoperative management of LEGG-PERTHES-C.ALVE disease.

\section{SPACE 1729}

Color flow and duplex Doppler imaging of the ductus venosus in neonates

N. Loberant (Presenter), D. Gaitini, M. Barak, M. Herskovits, M. Ben-Elisha, N. Roguin; Nahariya

Purpose: To study the anatomic and physiologic characteristics of the ductus venosus in a population of normal neonates, and to establish the time curve for ductus closure in this population.

Methods and Materials: 56 healthy newborn infants were examined with B-mode, color flow, and duplex Doppler imaging at age 1-2 days. Those with a patent ductus venosus were examined every 3 days thereafter until ductus closure was ascertained, or until age 14 days.

Results: The ductus venosus was patent in $100 \%$ of infants at $1-2$ days; in $60 \%$ at $6-7$ days; $22 \%$ at $9-10$ days; $7 \%$ at 14 days.

We present pictorial representation of the ductus with the modalities listed above and graphic representation of the time curve of ductus closure.

\section{SPACE 1730}

\section{The Sturge-Weber syndrome: MRI-enhanced findings}

L. Marti-Bonmati (Presenter), F. Menor, C. Poyatos, J. Vilar, H. Cortina, F. Mulas; Valencia

The Sturge-Weber syndrome is a neurocutaneous syndrome that usually associates facial venous angioma and leptomeningeal angioma

We studied 14 consecutive cases, 8 of them with Gadolinium (Gd-DTPA) enhanced MRI, with an age of $11.6 \pm 4.9$ years. MRI (Philips Gyroscan S5) was done with long TR/long TE Spin-Echo (T2WSE) (TR $=2200-2500$ $\mathrm{msec} / \mathrm{TE}=50,100 \mathrm{msec} / 2$ signal averaging), and $\mathrm{Tl}$ weighted Gradient-Echo $(\mathrm{TlWGE})(\mathrm{TR}=569 \mathrm{msec} / \mathrm{TE}=16 \mathrm{msec} /$ angle $=90 / 2$ signal averaging $)$ after Gd-DTPA administration.

All patients showed gyral contrast enhancement, even when the CT study did not show any. This enhancement allowed to differentiate the leptomeningeal angioma from prominent sulci, that is difficult in T2WSE, and to delineate the full extent of the disease - in 1 case an affected temporal lobe was not noticed in the CT study. It also permitted to demonstrate the anomalous vessels of the occasionally associated venous angioma, and to differentiate between this venous anomalies and prominent draining veins. The combination of TIWGE and Gd-DTPA permitted to detect calcifications (GE technique) and patency of the angioma (Gd-DTPA administration).

MRI, with T2WSE and TIWGE contrast enhanced, is the imaging modality of choice in the management and follow-up of these patients. C.T should be used only if the MRI study is negative, to rule-out characteristic cortical calcifications. 


\section{SPACE 1731}

Effectiveness of pneumatic reduction of lleocolic intussusception in children

F. Menor (Presenter), H. Cortina, R. Olague, D. Muro, C. Alberto, J. Lopez, A. Marco, A. Vallcanera, V. Andres, R. Aparici; Valencia

Intussusception reduction by rectal insuflation of air with manual controlled pressure is a simpler, faster and more successful alternative than the classical hydrostatic enema.

A total of 158 therapeutical enemas were performed to 133 patients ( 16 patients with one or more recurrent episodes). Perforation and/or an important impairment of the children's general condition were the only main contraindication for the reduction attempt. An appropiate-sized (18-20 F) Foley catheter, with $20-25 \mathrm{cc}$ of ballooning into the rectum, is connected to a sphyngomanometer to control the pressure administered $(80-120 \mathrm{~mm} \mathrm{Hg}$ ); no previous pharmacologic treatment was used. An almost always sudden small bowel aeration results in reduction success.

Up to date 141 successes (89\%) with no complications were achieved. In $16 \%$ of the patients, following pneumatic reduction, a thickening of the ileocecal valve showing a multiple appearance was observed. This fact sometimes results in a differential diagnosis with incomplete reduction; when small bowel aeration is observed, it has always been a sign of complete reduction, no false positive has been found.

We have undoubtedly improved the previous success rate at our Hospital with the hydrostatic reduction ( $79 \%$ ) as well as we have attained a simpler and faster technique for intussusception reduction in children.

\section{SPACE 1732}

Multicystic encephalomalacla in liveborn twin with a stillborn macerated co-twin

A. Ramos (Presenter), M. Miralles, A. Medina, G. Pozo, F. Mateos, R. Simon; Madrid

Twin gestations are at high risk of developing serious complications. When a twin has died in utero thromboplastine like material and/or debris from the died fetus are released to the circulation of the surviving co-twin. This fact may lead to multiple embolic ischemic events. In the central nervous system (CNS) the type and severity of the structural defect which may follow are intimately related to the gestational period when the CNS has been damaged. Four different lesions have been described in the brain including hydranencephaly, ischemic infarcts, multicystic encephalomalacia and porencephaly.

We report the wide spectrum of radiological and clinical findings in 10 cases of twin embolization syndrome with severe neurological involvement including 2 cases with evolutive lesions. CT was available in all our cases and ultrasound in 6 patients. A MR was performed in one patient.

\section{SPACE 1733}

The fetal profile, an aid in the diagnosis of fetal anomalies P. Twining (Presenter); Nottingham

Purpose: In order to assess the significance of the facial abnormalities associated with congenital anomalies, a three year review was made of all fetuses referred for a detailed scan at Queen's Medical Centre, Nottingham. Methods: Detailed scans are usually carried out on fetuses at 18 to 20 weeks gestation. A full anatomical assessment is made and in addition where possible transverse, coronal and profile views of the fetal face are obtained. All antenatal scans were reviewed as were pathological specimens and postmortem reports.

Results: Facial abnormalities were seen in 20 fetuses. The most common finding was 6 cases of micrognathia and this was present in 4 out of 9 fetuses with Trisomy 18, one case of camptomelic dysplasia and one case of short rib polydactyly syndrome.

The most striking appearances were seen in the 6 cases of alobar holoprosencephaly. 5 cases showed facial abnormalities ranging in severity from cyclopia with proboscis, cebocephaly, to hypothelorism, midline clefting and nasal hypoplasia. One case however demonstrated normal facial structures.

Facial clefting was present in 2 cases of Trisomy 13 and one case of Roberts Syndrome. Depression of the nasal bridge was seen in one case of thanatophoric dwarfism and typical facial appearances were seen in the 5 cases of anencephaly.

Conclusions: We feel the visualization of the fetal face is essential in the assessment of intracranial abnormalities and in any situation where a chromosomal abnormality is suspected. It also provides additional information in the assessment of skeletal dysplasias.

The poster will be presented in the form of the front page of that well known newspaper The Daily Scanner. The headline will read: "The fetal profile, have you seen these faces?"
SE Contrast media (10)

SPACE 1734

A comparison of the effects of lotrolan and Diatrizoate on the function of the Isolated perfused rat kidney (IPRK)

P. Brown (Presenter), J. Haylor, A. M. El-Nahas, S. K. Morcos; Sheffield

The effects of iotrolan $(\mathrm{n}=4)$ a water soluble non ionic dimer $(300 \mathrm{mgl} / \mathrm{mL}$, osmolality $\left.320 \mathrm{mosmol} / \mathrm{KgH}_{2} \mathrm{O}\right)$ and sodium-meglumine diatrizoate $(\mathrm{n}=4)$ an ionic monomer $\left(300 \mathrm{mgl} / \mathrm{mL}\right.$, osmolality $\left.1600 \mathrm{mosml} / \mathrm{KgH}_{2} \mathrm{O}\right)$ on renal function were studied using the IPRK. The model permits the evaluation of the direct renal effects of contrast media independent of their systemic influences. Kidneys from adult male wistar rats were perfused at constant pressure $(100 \mathrm{~mm} \mathrm{Hg})$ in a recirculating system with $150 \mathrm{~mL}$ of perfusate based on Krebs-Henselit solution. After a $\mathbf{4 0}$ minute equilibration period, the renal functional parameters were measured over 10 minutes control period. $10 \mathrm{~mL}$ of the contrast medium was then added to the perfusate reservoir and further measurements were taken during the next 30 minutes experimental period. Both agents induced significant $(P<0.05)$ reduction in glomerular filtration rate (GFR), renal perfusate flow (RPF) and a marked increase in the fractional excretion of albumine (FEAlb). With iotrolan the changes were GFR - 39\%, RPF $-24 \%$ and FEA Ib $+341 \%$, with diatrizoate they were GFR $-40 \%$, RPF $-27.7 \%$ and FEA1b $+193 \%$. The functional parameters of kidneys perfused without the addition of contrast agents remained stable throughout the control and experimental period.

In conclusion iotrolan and diatrizoate at the tested doses induced significant impairment of renal function. The effects are comparable in spite of the marked difference in their osmolality.

\section{SPACE 1735}

lomeprol $300 \mathrm{mg} \mathrm{l/ml} \mathrm{Vs} \mathrm{lopamidol} 300 \mathrm{mg} \mathrm{l} / \mathrm{ml}$ in conventional arteriography of cerebral arteries: phase III comparative study N. Caputo (Presenter), E. Ciorba, G.P. Pelliccioli, P. Floridi, R. Luccioli, R. Scaroni, E. Signorini; Perugia

Purpose: To compare safety and efficacy of Iomeprol and Iopamidol $(300 \mathrm{mg}$ $\mathrm{I} / \mathrm{ml}$ ) in conventional cerebral arteriography.

Methods and Materials: Forty-eight patients (age between 21 and 77 years) were randomized to double blind treatment with 24 subjects receiving Iomeprol and 24 Iopamidol. Criteria for inclusion were hospitalization, indication for conventional cerebral arteriography, written informed consent, age over 18 years, negative pregnancy test and contraception, clinical, laboratory and instrumental tests before procedure. Physical and neurological evaluation, laboratory tests, ECG and EEG were performed at the entry, 24 and 72 hours after procedure. Subjective and objective tolerability were assessed, side effects monitored and contrastographic efficacy evaluated.

Results: In the lomeprol group 86 injections were performed and in the Iopamidol 93 by i.a. Seldinger technique. The mean total volume of CM injected was comparable between groups: $102.4 \mathrm{ml}$ (Iomeprol) and 90.4 (Iopamidol). Efficacy was scored excellent for all $x$-rays technically adequate ( $95 \%$ Iomeprol and $98 \%$ Iopamidol). Results of the patient assessment using a $100 \mathrm{~mm}$ visual analogue scale indicated that, in general, pain and heat sensations were minimal or moderate (medians were $0-10 \mathrm{~mm}$ for pain and $50-60 \mathrm{~mm}$ for heat in both groups). Similarly, evaluation of patient tolerance by the investigator showed that only one patient had evidence of distress (score 1) during $2 / 4$ injections in the Iopamidol group. No side effects were reported. Conclusions: We can conclude that both contrast media, in this population, provided excellent opacification, were well tolerated by most of patients and had little effect on laboratory and instrumental safety parameters.

\section{SPACE $\underline{1736}$}

Urography with non ionic contrast media and thyroid function in newborns

C. Defilippi (Presenter), G. Masi, G. Di Rosa; Torino

In the last years screening programs allowed the recognition of a relatively high percentage of newborns with hypothyroidism that had undergone exposition to or excess of iodine during pregnancy or in the early days of life.

The intravenous administration of hydrosoluble contrast media require high quantities of iodine. We studied the thyroid functionality through RIA dosage of T3, T4, TT4 e TSH in a group of 10 newborns that underwent intravenous pielography (IVP) through injection of $5-7 \mathrm{mml} / \mathrm{kg}$ of hydrosoluble non ionic contrast media (lopamidolo).

The evaluation of thyroid function tested at 10 and 30 days from IVP did not show significant changes. According to our experience the administration of non ionic hydrosoluble contrast media in newborns does not yield a block of the thyroid function caused by excess of iodine, which - on the contrary - is a current problem if traditional contrast media are used. 
Renal tolerance of loxaglate in patients with chronic renal failure G. Deray (Presenter), C. Jacquiaux, M. Drobniski, M. C. Jaudon, C. Jacobs; Paris

The aim of the present investigation was to precise the renal tolerance of Ioxaglate in patients with chronic renal failure. Eight male patients (mean age $55 \pm 5$ years) with chronic renal insufficiency (glomerular filtration rate less than $60 \mathrm{ml} / \mathrm{mn}$ ) undergoing diagnostic cardiac catheterization were enrolled in this study.

Renal clearance study (inulin and PAH clearances) and enzymuria were carried out one day before and one day after Ioxaglate administration. The amount of contrast medium which was injected was $167 \pm 43 \mathrm{ml}$. None of the patients experienced any adverse reactions. All the patients had markedly depressed renal clearances values before angiography. Mean serum creatinine, glomerular filtration rate (GFR), effective renal plasma flow (EPRF) and urinary beta-2 microglobulin excretion were unaltered by angiography. Only one patient had an increase in serum creatinine of more than $10 \%$ (from 136 to 156 micromoles/l; 1.3 to $1.8 \mathrm{mg} / \mathrm{dl}$ ) after the procedure with a decrease in GFR from 34 to $27 \mathrm{ml} / \mathrm{mn}$. In this patient serum creatinine and glomerular filtration rate normalized within 72 hours.

Using accurate and sensitive renal function tests we have thus shown that Ioxaglate may be safely used in patients with chronic renal failure.

\section{SPACE 1738}

$\mathrm{CO}_{2}$ gas digital substraction angiography: an alternative to nonionic contrast material

Irvin F. Hawkins, Philippe P. Giacomino (Presenter), Mark Hawkins, Robert Hirko; Gainesville, FL

lodinated contrast continues to be the gold standard for vascular diagnosis. Even with the introduction of nonionic material, renal toxicity and mortality still occur. We have used $\mathrm{CO}_{2}$ gas below the diaphragm as an intravascular contrast agent in over 100 animals and over 600 patients. Diagnostic results were comparable to iodinated contrast in the majority of patients. The gas, buoyant agent, totally displaces the column of blood, especially if the organ of interest is placed higher than the injection site, then is eliminated by the lungs in a single pass. Results have been best in the lower extremities since bowel gas motion does not degradate the image. They were recently improved by the use of: (1) a dedicated injector permitting reliable delivery of this compressible agent, and (2) occlusion catheters. No significant complications have occurred. The paper will also compare the advantages and disadvantages of $\mathrm{CO}_{2}$ to the newer nonionic contrast agents. The advantages of $\mathrm{CO}_{2}$ are: (1) no allergy or apparent renal toxicity, (2) low viscosity permitting delivery via very small catheters. (3) unlimited total volumes since $\mathrm{CO}_{2}$ is eliminated by the pulmonary circulation, (4) minimal or no discomfort, and (5) cheapness.

\section{SPACE 1739}

Pathophysiological changes of the lung induced by intravenous administration of contrast media - experimental study in the rat

H. Hayashi (Presenter), T. Kumazaki, A. Yamamoto, M. Onda, R. Takagi, G. Asano, K. Ebata; Tokyo

Purpose: To investigate the lung weights and histological changes induced by intravenous administration of ionic and nonionic contrast media in rats. Methods and Materials: A comparative study of non-treated, physiological saline, Diatrizoate, lohexol and loxaglate was carried out using male wister rats. Each contrast medium prepared in solution containing $6 \mathrm{~g}$ iodine per $\mathrm{kg}$ or corresponding volume of saline was administered at a rate of $1.5 \mathrm{~mL}$ per minute. Animals were necropsied 20,60, and 120 minutes after the administration of contrast media. The lung was removed and weighed. Histological specimens were analyzed for further investigation of microscopic changes.

Results: Although the lung weights were significantly increased after intravenous administration of all contrast media, no statistical significance was obtained in each material. Perivascular edema and dilatation of hilar lymph vessels were histologically confirmed in the contrast media groups. There was no statistical significance of perivascular edema gradings in each material. There were well proportional correlation between increased lung weights and perivascular edema gradings and dilatation of hilar lymph vessels gradings.

\section{SPACE 1740}

The cllnical experience with Optiray 300 and 350 in head and body CT H.-M. Hoogewoud (Presenter), A.-C. Neiss; Fribourg

OPTIRAY (ioversol) is a new, non ionic, monomeric low osmolar contrast medium (LOCM) with a high degree of hydrophilicity.

The aim of this presentation is to report the results of a randomised parallel group study comparing OPTIRAY 300 and ULTRAVIST 300 in body CT on
80 patients that has been performed at the Fribourg hospital (Switzerland). The diagnostic efficacy has been assessed by rating the scan quality as poor, fair, good or excellent and in 4 studies on a visual analogical scale.

The safety was assessed by observation for adverse events after the injection of ioversol.

The quality of the procedure was rated good or excellent in nearly all the cases. This study also demonstrated good overall tolerance, with no major adverse reactions encountered. The results of this study are included for discussion in the whole European experience with OPTIRAY 300 and 350 in head and body CT, which includes 15 clinical trials (total 683 patients, 6 open-label studies and 1 comparative study in head CT; 5 open-label studies and 3 comparatives studies in body $\mathrm{CT}$ ).

It is concluded that OPTIRAY is an effective and well-tolerated contrast agent when used in contrast enhanced CT.

\section{SPACE 1741}

Optimization of contrast media application using doubleperistaltic-infusion pump for CT and MRI

Michael V. Knopp (Presenter), Hans-Ulich Kauczor, Stefan Delorme, Bruno Betsch, Günther Layer, Wolfhard Semmler, Alois Knopp, Gerhard van Kaick; Heidelberg

Purpose: To develop a reliable, standardized technique of contrast media application for CT and MRI with the use of a double peristaltic infusion pump. Greatest emphasis had to be placed on practicability, reliability and lowest financial cost.

Methods and Materials: A double peristaltic infusion pump designed for intensive care treatment with a flow rate of $1-99 \mathrm{ml} / \mathrm{min}$. was found to be the best practical choice. This pump was used in conjunction with a remote control. The original infusion bottle with the supplied infusion line was used with a "Braunüle" for i.v. puncture. CT contrast media was prewarmed to body temperature.

Results: In an initial evaluation the reliability and practicability of the infusion pump was evaluated. We found a reliability of $99 \%$ in summary of all different procedures. Depending on the location of the patient no additional recurrent cost in addition to the supplied infusion line were realised except an additional extension line as necessary. Quantitative evaluation of contrast enhancement measured by H.U. showed an average enhancement of $348 \%$ compared to $280 \%$ using a manual bolus technique. The time of contrast in vascular structures in the thorax was prolonged by at least $35 \%$. The contrast enhancement as well as contrast homogeneity were significantly improved in studies of the thorax, head and neck, liver as well as pelvis.

Conclusions: A double peristaltic infusion pump enables optimized application of contrast media for CT and MRI, improving enhancement, contrast homogeneity as well as time of contrast without any additional recurrent cost.

\section{SPACE 1742}

Magnetic resonance functional imaging of cerebral perfusion in the rabbit

C. Louail (Presenter), H. Trillaud, N. Grenier, J. M. Franconi, W. Kawiecki, J.M. Caillé; Bordeaux

The evaluation of cerebral perfusion by magnetic resonance imaging was impossible until now, because of the very long sequence acquisition time. The purpose of the present study was to develop a technique for the evaluation of cerebral perfusion with MRI, using technological progresses in the rapid acquisition of sequences (TURBO FLASH) and the original pharmacokinetic properties of a new contrast product, AMI 25 (diffusion strictly limited to the intravascular space, plasmatic epuration by capture by the reticuloendothelial system).

The study was performed in two steps:

1. an in vitro study to define the TURBO FLASH T2-weighted sequence and select the doses to be used in vivo.

2. an in vivo study to evaluate the variations in signal intensity of the cerebral parenchyma of the rabbit, after an I.V. bolus injection of super-paramagnetic particles (AMI 25).

The signal loss of the cerebral parenchyma due to transit of the contrast medium was determined by the ratio between the measure of R.O.I. and the background noise.

A curve of the decrease of the signal over time was performed, using normal rabbits as well as a pathology model of cerebral ischemia, to demonstrate the interest of this technique in the early diagnosis of cerebral ischemia. 
Leukotrienes (LT's) plasma levels during radlographic contrast medla (r.c.m.) reactions

C. Pozzato (Presenter), M. Gardinali, L. Conciato, A. Calcagno, F. Gattoni, M. Blanc, U. Baldini, M. di Bello, A. Agostoni, C. M. Uslenghi; Milan

Infusion of r.c.m. is followed in $\mathbf{4 - 8 \%}$ of cases by reactions whose pathogenic mechanism is still not well understood. Recently an involvement of Lt's, biologically active arachidonic acid metabolites in these reactions has been proposed since their biologic activity resembles some of r.c.m. reaction symptoms.

We measured therefore $\mathrm{LTB}_{4}$ and peptido-Lt's $\left(\mathrm{LTC}_{4}-\mathrm{LTD}_{4}-\mathrm{LTE} \mathrm{C}_{4}\right)$ levels in 20 subjects undergoing intravenous urography with two different low-osmolarity r.c.m.: ioxaglate (ionic dimer) and iopamidol (non ionic). Lt's levels were measured by radioimmunoassay after silica column nonpolar extraction from plasma.

$\mathrm{LTB}_{4}$ levels $(\mathrm{ng} / \mathrm{ml})$ before infusion of r.c.m. were $1.46 \pm 0.37$ and $1.31 \pm 0.30 \mathrm{in}$ patients treated with ioxaglate and iopamidol respectively. Peptido-Lt's pre-infusion levels $(\mathrm{ng} / \mathrm{ml})$ were $6.07 \pm 2.96$ and $6.39 \pm 2.48$. Neither significant variation in $\mathrm{LTB}_{4}$ and peptido-Lt's levels nor evidence of their biologic activity (leukopenia or change in blood pressure) was observed 1-5-15 minutes after r.c.m. infusion.

Three ioxaglate-treated subjects mildly reacted to infusion (urticaria, nausea, vomiting) without any significant modification of LT's levels.

We conclude therefore that Lt's are not released during r.c.m. infusion and are not responsible of reactions to contrast agents.

\section{SPACE 1744}

ECG changes during intrauterine injection of lonic contrast medium A. Prober (Presenter), A. Marmor, S. Chait, E. Schaeffer; Safed

The effect of intrauterine injection of meglumine-sodium-diatrizoate- $60 \%$ on ECG was studied during routine hysterosalpingography (HSG) in 28 young healthy women.

12 Lead computerized ECG was recorded at baseline, during instrumentation, immediately after intrauterine injection of up to $20 \mathrm{cc}$ of contrast medium (CM) and 2-3 minutes thereafter (late phase). In order to rule out a mechanical dilatatory effect of the $\mathrm{CM}$, up to $20 \mathrm{cc}$ of saline were injected prior to $\mathrm{CM}$ in 10 women, serving as a control group.

The most important single finding observed was significant QTc prolongation in $25(90 \%)$ out of the 28 women examined. QTc increased from $417 \pm 18 \mathrm{msec}$ to $451 \pm 30 \mathrm{msec}(\mathrm{P}<0,001)$. This effect disappeared within 2-3 minutes, QTc returning to the baseline values $(414 \pm 18 \mathrm{msec})$. The $\mathrm{QTc}$ did not change significantly after saline injection $(418 \pm 18 \mathrm{msec})$.

The mechanism causing the QTc prolongation is unclear. Anxiety is obviously not the cause as QTc changed only during $\mathrm{CM}$ injection.

Dilatation of the uterine cavity was ruled out due to the fact that saline injection did not cause the same effect. A plausible explanation may be the local endometrial effect with neuro-vegetative reaction.

\section{SPACE 1745}

A multicentre study of Omnipaque ${ }^{\oplus}$ in urography, comparing plastic (polypropene) containers to glass vials

A.-N. Dardenne, J. Fairhurst, J. Grellet, G. Jenssen, T. Kristoffersen, L. Lemaitre, K. Skinningsrud (Presenter), R. Svihus, K. Tveit; Oslo

The purpose of the trial was to compare the general tolerance of iohexol (Omnipaque $\left.{ }^{\circledast}\right) 350 \mathrm{mg} \mathrm{I} / \mathrm{ml}$ filled in polypropene containers and in routinely used glass vials by recording adverse events before, during and up to one hour after injection. Allergy like reactions (coughing, sneezing, nausea, vomiting, urticaria and itching) were especially focused upon.

The trial was designed as a randomized double-blind parallel multicentre trial performed in 6 hospitals, representing 4 countries. Premedication, known hypersensitivity and use of compression was recorded. Patients with known previous serious reactions to contrast media were not included. Patients in the Norwegian and English hospitals received $50 \mathrm{ml}$ and patients in the Belgian and French hospitals received $100 \mathrm{ml}$ of contrast medium. Totally 1482 patients were included.

Fourteen of 740 patients in the polypropene group (1.9\%) and 15 of the 742 patients in the glass vial group $(2.0 \%)$ had allergy like reactions with no difference between the centres. No difference was found in the frequency of allergy like reactions between the groups compared. Neither was a difference found in the frequency of adverse events in general.

We thus conclude that polypropene is suitable as a container material for Omnipaque ${ }^{\oplus}$.
The pharmacodynamics of the vascular MR contrast agent Gd-DTPA Polylysine in the rabbit and the rat

P. Van Hecke (Presenter), G. Marchal, H. Bosmans, K. Johannik, H. Vogler, Y. Jiang, A. L. Baert, U. Speck; Leuven

Purpose: MRI study of the pharmacodynamics and of the vascular enhancement capability of Gd-DTPA Polylysine in the rabbit and the rat. Methods: MRI was performed at $1.5 \mathrm{~T}$ using a SE sequence $(300 / 15 \mathrm{~ms})$. Signal intensities of the liver, kidneys and muscle were measured before and up to 2 weeks after injection of $0.1 \mathrm{mmol} / \mathrm{kg}$ Gd-DTPA Polylysine in the rabbit $(\mathrm{n}=$ $3)$ and the rat $(n=3)$. Additional rabbits were injected with $0.04,0.2$ and $0.4 \mathrm{mmol} / \mathrm{kg}$ to study the dose response. A 3-D inflow FISP MRA sequence was used to image the vessels.

Results: The pharmacodynamics are similar in the rabbit and the rat. The liver intensity is enhanced by $60 \%$ and decreases with a half life of about $60 \mathrm{~min}$. The kidney medulla and cortex are enhanced by much larger factors ( 3 to 4 ) and the half life is about one day. The muscle intensity is not significantly affected. The $0.1 \mathrm{mmol} / \mathrm{kg}$ dose is optimal for the liver since $\mathrm{T} 2$ effects predominate at higher concentrations.

Superb angiogrammes of the rabbit posterior limbs were obtained; submillimeter arteries and veins were visualised over the entire length of the limbs even at the low dose of $0.02 \mathrm{mmol} / \mathrm{kg}$.

Conclusions: Gd-DTPA Polylysine is a promising agent for MR contrast and MRA studies.

\section{SE}

\section{SPACE 1747}

Pulse sequence optimization in 100 patients with urinary bladder carcinoma at $1.5 \mathrm{~T}$

Jelle O. Barentsz (Presenter), Sjef H.J. Ruijs, Leon J.T. O. van Erning, Chris J. G. Bakker; Nijmegen

Purpose: To determine the optimal pulse sequence(s) for staging urinary bladder cancer (UBC).

Methods: In 14 patients with UBC T1 and T2 values of perivesical fat, bladder wall, urine, and tumor were estimated. On the basis of these values contrast matrices were calculated, from which the sequences with optimal contrast between these structures could be derived.

Additionally, the changes in contrast between these structures were illustrated by synthetic imaging. To confirm the value of the found pulse sequences in Staging of UBC, they were applied for 100 patients with UBC.

Results: Optimal staging of UBC requires at least 2 pulse sequences:

1. A TI weighted pulse sequence $(\mathrm{SF} / 750 / 15)$ is optimal to determine tumor invasion in perivesical fat, and to recognise enlarged lymphnodes and bone marrow infiltration.

2. A T2 weighted pulse sequence $(S F / 2100 / 30,100)$ is optimal to determine tumor invasion in bladder wall, prostate, seminal vesicles or uterus, and to differentiate between tumor and fibrosis.

The STIR sequence (IR/2000/175/60) is superior to the SF/2100/30, 100 sequence to recognise bone marrow metastases.

Conclusions: Optimal pulse sequenced for staging UBC are $\mathrm{SE} / 750 / 15$ and $\mathrm{SF} / 2100 / 30,100$. In bone marrow metastases the $I R / 2000 / 175 / 60$ sequence has advantages over the $\mathrm{SF} / 2100 / 20,100$ sequence.

\section{SPACE 1748}

\section{Disseminated cerebral tuberculosis}

P. Benz (Presenter), A. Seeliger, G. Hamann, B. Junk, U. Hau, C. Kujat, W. Dewes; Homburg/Saar

We report about a 40 year old patient who presented with febril temperature and left sided hemiparesis. In the history there was a lung resection because of tuberculosis 20 years ago. One of the diagnostic examinations was the MR-tomography of the brain. Supra- and infratentorial multiple circular lesion with central necrosis, perifocal edema and marginal contrast enhancement were seen. The histological result of the stereotactic biopsy represented a granulomatosis inflammation with central necrotic areas. There was no sign for metastasic genesis or other inflammations. Under tuberculostatic therapy there was a good regression of the radiological and clinical findings. Therefore retrospectively the diagnosis of cerebral tuberculosis is verified.

This case does not agree with the well known manifestation of cerebral tuberculosis.

MR-images demonstrate very clearly the exact location of the lesions and their regression after therapy. 


\section{SPACE 1749}

\section{Usefulness of MRI in cystic tumors of the pancreas}

F. Boudghène (Presenter), D. Mathieu, P.J. Valette, D. Solvit, P.M. Deslandes, J. M. Bigot; Paris

Ten patients with cystic pancreatic neoplasms, were explored on MRI after US and CT examination. Several criterias as signal intensity modifications of morphologic aspects were studied, for expose specific findings.

These examinations were performed on a low field magnet in 4 cases, and on a high field magnet in 6 cases, with transversal slices on these lesions in $\mathrm{Tl}$ and $\mathrm{T} 2$ sequences.

Those patients had serous cystadenomas in 3 cases, and mucinous tumors in 4 cases, but cystadenocarcinomas in 3 other cases. MRI allowed only measurements of these cysts and particularly their size, number, and also the thickness of their septas. However signal intensity, neither homogeneity of these cysts, were at all specific of a kind of tumor, or even different of some pancreatic pseudocyst.

Diagnosis was essentially based on morphologic criterias well known, such as more than 6 lesions of less than $2 \mathrm{~cm}$ for serous cystadenomas, and as less than 6 cysts of more than $2 \mathrm{~cm}$ for mucinous lesions. This study showed also, that signal modifications on MRI in T1 and T2 sequences are not specific, and not sufficient for differentiated histologic type of cystic pancreatic tumors.

\section{SPACE 1750}

MR follow-up evaluation in conservative treatment of lumbar disk herniation

A. Bozzao (Presenter), M. Gallucci, L. Aprile, B. Orlandi, A. Cifani, R. Passariello; L'Aquila

Spontaneous regression of disk herniation in the lumbar spine has already been described as well as reduction or disappearance of acute back pain or sciatica after conservative treatment. None correlated on follow-up studies disk herniation evolution to clinical course of the disease. Moreover there are not series of patients followed after the radiological diagnosis of disk herniation large enough to assess a possible statistical analysis about the natural evolution of this disease. In the course of $1116 \mathrm{MR}$ examinations performed in our Institution in the past three years the diagnosis of disk herniation was made in 286; out of these, 81 underwent the follow-up MR, performed 6 to 15 months after the first one. A volumetric evaluation of disk herniation was performed on sagittal and axial planes. Based on the modification of the volumetric values obtained in the two MRs we classified the patients in 4 groups. The clinical evaluation was performed using a method stated by Pearce and Moll. Our data showed a high incidence of regression of herniated material $(63 \%)$, a low incidence of progression ( $8 \%$ ) and a tight correlation between morphologic and clinical findings.

\section{SPACE 1751}

\section{MR evaluation of abdominal and retroperitoneal masses in children} M. Cerioni (Presenter), R. Lanza; Ancona

Magnetic resonance imaging (MRI) has been used to evaluate 15 children with abdominal or retroperitoneal masses.

All exams were performed with a $1.5 \mathrm{~T}$ superconductive magnet (Philips Gyroscan). Sedation was regularly used in patients under 6 years of age.

Correlation of the MRI findings with Computed tomography (CT) was obtained in 8 children and with surgical findings in 10 .

MR accurately detected the lesions and their extent in all patients. It correctly evaluated the possibility of resectability and response to treatment.

MR appears to be a reliable technique for the diagnosis, staging and follow-up of children with abdominal and retroperitoneal masses.

\section{SPACE 1752}

Factor analysis of fast dynamic MR imaging in transplanted kidneys Joel Chabriais (Presenter), Olivier Hélénon, Jean-Michel Correas, Habib Benall, Christophe Legendre, Frederique Frouin, Jean-Francois Moreau, Robert Di Paola; Paris

Purpose: To obtain renal and urinary excretion factors by Gd-DOTA Dynamic MRI (DMRI) and using Factor Analysis of Dynamic Structure (FADS) to follow-up renal transplants.

Methods and Materials: We examined 6 renal transplanted patients using DMRI after a Gd-DOTA bolus $(0.2 \mathrm{ml} / \mathrm{kg})$ : ten to twelve fast gradient-echo sequences ( $\mathrm{TR} / \mathrm{TE}=40 / 14$, flip angle $=45^{\circ}$, acquisition time $=13$ seconds) with single images and 32 second-intersequence delay. Out of these, 13 DMRI sequences are processed using an extension to DMRI images of FADS, previously developed to analyze Nuclear Medicine dynamic studies. The results are confronted to the results of biological dosages, renal biopsy and Seldinger digital arteriography.

Results: FADS extracts cortical, medullary and excretory factors. Each factor is represented by a curve which is an estimate of the underlying physiological function, a factor image which is the associated spatial distribution and a contribution which quantifies the relative importance. The 13 processed studies include 3 examinations from patients with transplant rejection histologically proved. Preliminary findings correlate positively with biological results and renal biopsy.

Conclusions: DMRI sequences FADS processing automatically extracts objective features (cortical, medullary, excretory factors) enabling to evaluate the renal cortex and medulla function.

\section{SPACE 1753}

Focal fatty infiltration of the liver mimicking metastatic disease: value of MAI

T. H. Dao (Presenter), D. Mathieu, T. Nguyen-Tan, S. Derhy, N. Vasile; Paris

Purpose: To assess the ability of MR imaging to identify focal fatty infiltration of the liver, and to differentiate this common pathologic change from metastatic disease.

Methods and Materials: 3 patients underwent MRI of the liver because of lesions detected on sonography or computed tomography. One patient had a clinical history of neoplastic disease (breast cancer); all patients are clinically suspected of metastatic disease. MRI examinations were performed with $1.5 \mathrm{~T}$ system (Signa, General Electric) with various spin-echo sequences.

Results: All lesions appeared hyperechoic on sonography, with low attenuation density on $\mathrm{CT}$, and a high signal intensity on $\mathrm{T} 1$ and $\mathrm{T} 2$ sequences, suggesting fat tissue.

Conclusions: In our experience, MRI can resolve confusion between focal fatty infiltration of the liver and metastatic disease.

\section{SPACE 1754}

Gd-DOTA enhanced MRI of renal tumors

A. Denys (Presenter), O. Hélénon, L. Rotkopf, E. Atlan, N. Lebo, Y. Chrétien, B. Dufour, J. F. Moreau; Paris

Purpose: The aim of this prospective study is to evaluate the contribution of Gd-DOTA in the MR diagnostic of renal tumors and for the staging of renal cell carcinoma.

Methods and Materials: We explored 20 renal cell carcinoma (including 4 cystic carcinomas) and 6 benign tumors. We performed $T 1$ and $T 2$ weighted SE sequences, and dynamic fast gradient echo sequences after bolus of Gd-DOTA.

Results: MR results has been correlated to macroscopic and pathologic datas $(26 / 26)$. Gd-DOTA injection does not improve the MR staging of renal cell carcinoma. Dynamic sequences seem to can differentiate good prognostic papillary carcinoma from clear cell carcinoma. Gd-DOTA provides a better analysis of the wall of cystic carcinomas. The characterization of benign indeterminate tumors is not improved by Gd-DOTA.

Conclusions: The use of Gd-DOTA does not improve the staging of renal carcinomas but is helpful to characterize cystic carcinomas and papillary carcinoma.

\section{SPACE 1755}

Can dynamic contrast-enhanced MRI Improve the differentiation of focal liver lesions?

Ch. Ehrenheim (Presenter), H. Petersen, K. F. Gratz, R. Weimann H. Hundeshagen; Hannover

Patients and methods: 35 patients with liver tumors (hemangioma, focal nodular hyperplasia FNH, hepatocellular carcinoma HCC, metastases) underwent MRI of the liver before and after i.v. bolus injection of $0,2 \mathrm{ml} / \mathrm{kg}$ Gadolinium-DTPA in addition to other dynamic imaging methods (HBSS, RBC scan using Tc-99m-labelled erythrocytes). Dynamic MR studies using a gradient echo sequence (FLASH) were done in addition to conventionally acquired T1- and T2-weighted images. Two different dynamic studies with various delay times were applied depending on the presumptive diagnosis. Results: Characteristic kinetics and uptake patterns were found: Liver hemangiomas show the most intensive uptake of Gd-DTPA. In the dynamic study the negative tumor-liver contrast first increases, and subsequently the lesion shows a delayed centripetal filling in. This facilitates the differentiation hemangioma/malignant tumor being similar in native images. The demarcation of FNH is usually not influenced by late contrast-enhanced images as it almost behaves like surrounding normal liver tissue: again dynamic MRI reveals the characteristic hyperperfusion, so that this combination of findings makes the diagnosis.

Finally, Gd-DTPA presents a mixed pattern of uptake and distribution in $\mathrm{HCC}$ and metastases with hyperperfusion as a common finding. The pos contrast images usually show a moderate inhomogeneous e.g. ring-shaped enhancement, again the combined findings giving the diagnostic hint.

Conclusions: Dynamic MRI is useful in the diagnosis of liver tumors and improves the differentiation of focal lesions. 
Dynamic Gd-DTPA MRI evaluation of breast lesions

P. Ercolani (Presenter), G.M. Giuseppetti, E. de Nigris, S. Baldassarre, A. Segoni, A. Giovagnoni; Ancona

Purpose: The purpose of this study is to define the diagnostic value of dynamic Gd-DTPA MR examination in the tissue characterization of breast lesion. Methods and Materials: 125 patients ( 53 years old) with different breast lesions (51 carcinomas, 18 fibroadenomas, 18 cysts and 44 treated with lumpectomy and radiotherapy) underwent $M R I$ investigation after a mammographic and US examinations. 51 breast cancers had histopathologic correlation and the others were followed up for 1 year. SE T1 - T2 pulse sequences and FLASH 2D were used. A dynamic enhancement series was obtained when nodular lesion was demonstrated. GE images $\left(100 / 12, \mathrm{FL}=70^{\circ}\right)$ were performed before and after the intravenous injection of Gd-DTPA $(0.2 \mathrm{~m} \mathrm{Mol} / \mathrm{Kg})$ at nineteen second intervals for eight minutes. Lesion parenchyma signal intensity ratio was calculated.

Results: All histologically proved carcinomas showed a significative increase of MR signal intensity within the first minutes after i.v. injection of contrast agent and nearly a plateau level afterwards. In cases of benign lesions dynamic behaviour of signal intensity showed a lower and slower increase in comparison with malignant lesion and a plateau level after 8 minutes. Our results indicate the diagnostic value of dynamic Gd-DTPA MRI in all cases with an undefined clinical and/or mammographic findings.

\section{SPACE 1757}

Computed tomography and magnetic resonance imaging of the chest after surgery for bronchogenic carcinoma

G. M. Favagrossa (Presenter); Leno

14 patients have been examined by Magnetic Resonance Imaging (MRI) and 13 by Computed Tomography (CT) of the chest in the follow-up after surgery for bronchogenic carcinoma. The patients were undergone to pneumonectomy (2), lobectomy (II) and segmentectomy (I) for: 10 squamous cell carcinomas, 3 adenocarcinomas, I small cell carcinoma.

MRI was performed using a 1,5-T system in all patients; MRI included TI- and $\mathrm{T} 2$-weighted sequences on axial views in all patients. $\mathrm{Tl}$-weighted sequences were performed also on coronal views in 10 patients and on sagittal views in 3 patients.

CT examination was performed from 15 to 3 days before MRI; one patient did not performe CT because of intolerance to contrast media.

MRI confirmed CT in 7 cases and was better in $6:$ in 3 cases of recurrence and in 3 cases of lymphoadenopathy of mediastinum.

MRI of the chest in the follow-up of bronchogenic carcinoma after surgery is useful when CT examination is not resolutive to identify a recurrence and/or a lymphoadenopathy of the mediastinum, and often to distinguish a recurrence from postirradiated tissue.

\section{SPACE 1758}

MR follow-up of cirrhotic liver: tissue characterization of carcinomatous and non-carcinomatous focal lesions at 1.5 tesla

F. Ferrozzi (Presenter), A. Rossi, R. Silva, L. Rossi; Parma

Purpose: with the current technical advances in imaging modalities, small focal lesions are now detected with relative case and increasing frequency in the cirrhotic liver follow-up. However the imaging findings of small $\mathrm{HCC}$ are often non-specific and the differentiation from non-cancerous spaceoccupying lesions, particulary adenomatous hyperplastic nodules, is difficult. We evaluated the role of MRI.

Methods and Materials: findings on 50 patients affected by liver cirrhosis examinated by MR 1.5 T unit are reported. TI (TR 350-500, TE 20-30) and T2 (TR 1800-2000, TE 80-100) SE sequences were performed. Results: 30 histologically proven small HCC $(\varnothing 15-35 \mathrm{~mm})$ are detected. All the lesions were hyperintense on $\mathrm{T} 2$-weighted images, while $\mathrm{Tl}$ images are not specific (hyperintense $5 / 30$, isointense $18 / 30$, hypointense $7 / 30$ ). All the 5 adenomatous hyperplastic macronodules detected were hypointense on T2 images $(2 / 5$ hyperintense, $3 / 5$ hypointense on $\mathrm{T} 1$ ). Regenerating siderotic micro-nodules are detected in $40 / 50$ patients in form of hypointense focal lesions only on $T 2$ images. The 2 false-negative cases are dependent to poor quality technique of the examen and a small size of the lesions $(10 \mathrm{~mm})$.

Conclusions: results obtained demonstrated that MRI is a very useful technique in the differential diagnosis of focal lesions in cirrhotic liver.
SPACE 1759

MRI evaluation of the pelvis after sphincter-saving anterior resection of the rectum

A. Giovagnoni (Presenter), P. Ercolani, M. Misericordia, R. Nozzolillo, E. de Nigris; Ancona

Purpose: The purpose of this work was to define the diagnostic possibilities of MRI in patient's monitoring after sphincter-saving anterior resection of the rectum.

Methods and Materials: 23 patients $(14 \mathrm{M}, 9 \mathrm{~F})$ who had undergone an anterior resection for recto-sigmoid carcinoma were studied. MRI investigation was performed by a tomograph 1.0 T (Magnetom). Multiplanar, multislice SE T1 and $\mathrm{T} 2$ pulse sequences were obtained. All patients were examined before and after retrograde colon filling using a paramagnetic contrast agent. 6 patients were studied after i.v. somministration of Gd-DTPA. 12 patients were studied 1 month post-up (group I) and 11 patients (group II) 6 months or more later from surgical treatment. MRI findings were correlated with traditional techniques and surgical or bioptic data.

Results: In group I, 6 patients demonstrated MRI abnormalities not visualized by traditional imaging modalities. In group II, MRI identified local recurrence in 4 patients and to define the fibrotic nature of a presacral process misinterpreted by CT in 2 cases.

Conclusions: MRI with contrast agent has proved its usefulness in follow-up after lower GI tract conservative surgical treatment.

\section{SPACE 1760}

MRI in pre-surgical evaluation of abdominal aortic aneurysm impending rupture

A. Giovagnoni (Presenter), P. Ercolani, M. Misericordia, F.P. Alò, L. Carbonari, E. de Nigris; Ancona

Purpose: Purpose of this study is to define the clinical value of MRI in pre-surgical evaluation of abdominal aortic aneurysm impending rupture. Methods and Materials: 30 patients $(23 \mathrm{M}, 7 \mathrm{~F})$ with abdominal aortic aneurysm impending rupture were studied. All patients underwent previously, abdominal US. MRI investigation was performed by a tomograph $1.0 \mathrm{~T}$ (Magnetom). One SE TI pulse sequence on axial plane as scout image (acquisition time $=22 \mathrm{sec}$ ) and two SE Tl pulse sequences on coronal and sagittal planes (acquisition time $=1.30 \mathrm{~min}$ ) were obtained. The investigation time resulted ranged between 10 to 25 minutes. All patients underwent surgical treatment ( $\mathrm{h}$. to $12 \mathrm{~h}$. from MRI investigation).

Results: MRI showed in 6 cases posterior parietal interruption with retroperitoneal bleeding, in 10 cases a subrenal short aortic collar and in 14 cases an aortic collar under the left renal vein. Renal aneurysmatic involvement was shown in 4 cases; in one case, autoctone dissection of abdominal, subrenal, aortic aneurysm was identified while in 3 cases inflammatory aneurysms misinterpreted by traditional methods as CT, US and angiography were correctly demonstrated. Examination time shortening allowed to utilize successfully MRI in the evaluation of aortic abdominal aneurysms impending rupture.

\section{SPACE 1761}

Colon enema with paramagnetic contrast agents in MRI evaluation of pelvic masses

A. Giovagnoni (Presenter), P. Ercolani, M. Misericordia, D. Migliorini, E. de Nigris; Ancona

Purpose: The purpose of this study is to define the diagnostic utility of colon enema with paramagnetic contrast agents in MRI evaluation of pelvic masses. Methods: 35 patients $(25 \mathrm{~F}, 10 \mathrm{M}$ ) with pelvic masses ( 10 ovarian, 12 uterine, 13 retroperitoneal neoplasms) proved by traditional methods (US, CT, BE) were studied. MRI investigations were performed before and after retrograde rectosigmoid filling by $300 \mathrm{ml}$ of paramagnetic contrast agent: 18 patients using Gd-DTPA acqueous solution $(1 \mathrm{mM}), 17$ patients using a home-made Fe-gluconate solution (JKAI). A superconductive 1.0 T Magnetom tomograph was used. Multiplanar, SE T1 and T2 images were obtained. MRI findings before and after colon enema were evaluated independently by two observers. MRI findings were correlated with surgical or pathological data.

Results: The colon enema with paramagnetic contrast agents provided a better identification of pelvic masses limits in $87 \%$ of cases, to evaluate in 4 cases colon infiltration misinterpreted with no contrast MRI investigation was correctly demonstrated. In 6 cases lymphnodes were easy distinguished by bowel wall when intestinal lumen was filled.

Conclusions: Our experience suggests the routine use of colon enema with paramagnetic contrast agents in MRI of the pelvis. 
Small MR imager and NMA spectrometer

G. Gomiscek (Presenter), E. Moser, A. F. Fercher, J. Pirs, B. Marin, V. Erzen, R. Blinc; Vienna

Magnetic resonance imaging and magnetic resonance spectroscopy are very important and promising techniques in biomedical applications. All the instruments developed so far (especially MR tomographs) are very sophisticated and expensive. In addition, very often they are working like a "black box" for the clinical user.

A simple, low cost NMR spectrometer and MR imager was developed. It includes a permanent magnet and a data acquisition system. In order to allow MR imaging, a gradient system is added, that can generate a linear magnetic gradient. The data acquisition unit has all the performances of a digital oscilloscope and can also communicate with an external computer. This communication allows the projection of live NMR signals of basic and imaging experiments on a large screen using a digital LCD overhead projector

Small MR imager and spectrometer allows the demonstration and practising of NMR techniques without occupying expensive professional NMR spectrometers and MR imagers for teaching purposes.

\section{SPACE 1763}

\section{Longterm quality control of MR imager/spectrometer}

G. Gomiscek (Presenter), H. Imhof, E. Moser; Vienna

Magnetic resonance imaging and spectroscopy became a powerful tool in modern medicine. However, the performance of this very complex diagnostic method is strongly depending on the machine optimization, hence on the quality control measurements.

The EC test objects (COMAC-BME II.1.3) were used in order to check the quality of MR tomograph. The test measurements were performed each year after its installation.

The first quality control discovered some failures which could be repaired and they did not repeat any more. It also showed a good quality of the instrument. Although the quality control measurements in the following two years confirmed its good performance, they also indicated some minor deviations from its initial status.

Our results show that these manufacturer independant quality control measurements are an additional guarantee for the optimal performance of the MR tomograph.

\section{SPACE 1764}

\section{${ }^{31} \mathrm{P}$ MR spectroscopy for diffuse liver disease}

Kumiko Ishizuka (Presenter), Hiroshi Ashida, Keiko Imamura, Atsushi Fukui, Mitsuaki Saeki, Tohru Ishikawa, Shogo Iwabuchi; Kawasaki

Purpose: Phosphorus-31 magnetic resonance spectroscopy (31P-MRS) of diffuse liver disease was performed and pattern of chronic hepatitis, alcoholic hepatitis and liver cirrhosis was investigated.

Methods and Materials: 3IP-MRS of the liver was performed on 8 healthy volunteers and 20 patients with diffuse liver disease. (1 case of acute hepatitis, 5 cases of chronic hepatitis, 5 cases of pre-cirrhosis, 9 cases of liver cirrhosis) Volume localization was fulfilled by using ISIS sequence. Peak values of phosphorus signals relative to $\beta$-adenosine triphosphate, intracellular hepatic $\mathrm{pH}$, signal intensity, pattern of spectra and comparison histological diagnosis with 3IP-MRS about some cases based on clinical diagnosis of chronic hepatitis or precirrhosis were evaluated.

Results: Chemical shift and $\mathrm{pH}$ show no significant differentiation in each group. Liver cirrhosis shows typical pattern of spectrum with attenuation of signal intensity and high phosphomonoester ratio. Alcoholic diffuse liver disease mimicked normal pattern of spectrum, which was though to become a non-invasive clue to differentiate from other type of diffuse liver disease.

\section{SPACE 1765}

\section{MR-imaging of an, agyria}

B. Junk (Presenter), A. Seeliger, P. Benz, U. Hau, W. Dewes, U. Piepgras; Homburg/Saar

Presented is a case with the rare malformaton of an agyria.

It is a 6 month old baby with a intrauterine diagnosed microencephalosis. After an unobtrusive development of pregnancy first in the age of 6 month BNS-convulsions appeared.

The MR-images are compared with the statement to the frequency and histology of this rare malformation in the literature.
High quality TOF-MR-angiography of the thorax: application of new acquisition procedures; presaturation pulses and postprocessing H.-U. Kauczor (Presenter), R. Hausmann, L. R. Schad, G. Layer, M. V. Knopp, W. Semmler, G. van Kaick; Heidelberg

MR-Angiography in the thorax is disturbed by motion artifacts (breathing, pulsation) and is thought to be of insufficient sensitivity.

Using the time-of-flight technique (1.5 Tesla Siemens Magnetom SP) sequence parameters were optimized for detection of intrapulmonary blood now. FLASH-2D gradient echo sequences with sequential scan acquisition (TR 30 $\mathrm{msec}$, TE $10 \mathrm{msec}, \mathrm{FA} 30^{\circ}, 1 \mathrm{Ac}$ ) in a 10 -second breath-hold period revealed to be most sensitive. Optimal slice thickness was found to be $5 \mathrm{~mm}$ with $1 \mathrm{~mm}$ overlap (matrix of $256 \times 256$ with oversampling, FOV $400 \mathrm{~mm}$ ). Frontal orientation results in the best overall contrast allowing the best delineation of the pulmonary trunk. Sagittal or oblique orientations are mandatory for hilar regions. Parallel travelling presaturation pulses in oblique orientation enable selective detection of pulmonary arteries and veins, whereas transverse presaturation avoids overlap of different vessels, making anatomical identification easier. An automized control procedure with instant image and individual continuation allows patient-monitoring and guarantees high quality angiograms. In MIP-postprocessing box size reduction and slice selection are used to create angiograms of interest (AOI) ensuring confident anatomical identification without overlap from neighbouring vascular structures.

This technique allows display of the mediastinal vessels, the hilar arteries and veins as well as the more peripheral intrapulmonary and intercostal vascular structures.

This excellent delineation and identification of vessels will be the base of successful application of MR-angiography in the thorax.

\section{SPACE $\underline{1767}$}

\section{Erdheim-Chester disease}

C. Kujat (Presenter), J.Martin, P.Benz, B. Junk, U. Hau, W. Dewes, U. Piepgras; Homburg, Saar

Erdheim-Chester disease is characterised by lipid granulomas in the long bones which cause characteristic radiological changes. Additionally, lipogranulomas can exist in multiple other organs, especially in lung, retroperitoneum and ZNS.

The characteristic radiologic findings are demonstrated by own observation.

\section{SPACE 1768}

Bone marrow MR imaging: assessment of contrast phenomena in hematopoletic hyperplasia

Philipp Lang (Presenter), Sharmila Majumdar, Russell Fritz, Martin Vahlensleck, Harry K. Genant; San Francisco, CA

Purpose: Hematopoietic hyperplasia is a frequent finding on MR imaging of the knee. We investigated the magnitude of the different relaxation processes such as chemical shift effects and inhomogeneous magnetic susceptibility contributing to the red-yellow marrow contrast on gradient-echo images.

Methods: MR imaging (1.5 T, GE Signa) was performed in 4 patients with known hematopoietic hyperplasia of the knee. Spinecho images were acquired with $\mathrm{TR}=600 \mathrm{msec}$ and TE $=20 \mathrm{msec}$. Gradient-echo (GRE) images were obtained with $\mathrm{TR}=250 \mathrm{msec}$ and $\theta=90^{\circ}$, TE was increased from $10-21 \mathrm{msec}$ at $1 \mathrm{msec}$ increments. Water-suppressed and fat-suppressed GRE images were generated with the same parameters using chemically selective presaturation pulses.

Results: Red-yellow marrow contrast on out-of-phase GRE images may be related to chemical shift effects or intracellular iron in hematopoietic marrow causing inhomogeneous magnetic susceptibility and intravoxel static field variation. Signal intensities of red and yellow marrow demonstrated an oscillating pattern with increasing TE on GRE images. Red-yellow marrow contrast was minimal on in-phase images (TE $=9,14,18 \mathrm{msec}$ ); it was maximal on out-of-phase images (TE $=7,11,16 \mathrm{msec}$ ). When the fat or water component were suppressed, red-yellow marrow contrast was not greater on out-of-phase GRE images than on in-phase GRE or spinecho images.

Conclusions: Improved red-yellow marrow contrast on out-of-phase gradient-echo MR images is primarily related to chemical shift effects resulting from the different fat and water content of hematopoietic and fatty marrow. Out-of-phase GRE imaging may be used to monitor distribution of hematopoietic marrow as a function of age and erytrocyte demand. 


\section{SPACE 1769}

MR and CT in the diagnosis of obstructive sleep apnea

M. Marotti (Presenter), V.Zjačić Rotkvić, V.Goldoni, Z. Tolić, V. Durigl, T. Čabrijan, T. Šušković; Zagreb

Magnetic resonance imaging and computerized tomography was performed in ten patients with obstructive sleep apnea syndrome proved by polysomnography. Computerized tomography have been established method for presenting oropharyngeal airway narrowing, which affects overweight men with hystory of loud snoring and disrupted sleep. The purpose of the study was to compare CT and MR transaxial slices in defining the degree of airway narrowing.

Computerized tomography, and magnetic resonance imaging (ultra-low field) was performed in ten patients (6 males, 43 females, mean age 45,13$)$ with obstructive sleep apnea syndrome proved by polysomnographic data and patient's history.

Cross-sectional area measurements of the narrowest part of airway in the oropharynx was made by drawing a region of interest on transaxial CT and MR slices. Results were expressed in square centimeters, and comparison of two methods discussed.

\section{SPACE 1770}

MRI in evaluation of Intrauterine cerebral fetal malformations

A. Baamonde, L. Marti-Bonmati (Presenter), C. Poyatos, J. Vilar, F. Menor; Valencia

The introduction of MRI in the evaluation of fetus is a recent achievement, with special interest in the evaluation of the Central Nervous System and its malformations. We consider this technique valuable not only in the morphological evaluation of the different cerebral structures, but in its capacity to certain the process of maturation and the distribution of myelin.

Our experiences of 13 cerebral fetal malformations include:

- 5 Hydrocephalias, 3 with neural canal defects, I unilateral.

- 3 Dandy-Walker malformations

-2 occipital encephaloceles

-2 aqueductal stenosis

-1 microcephalia of unknown cause

The MRI adds information in the study of the cerebral fetal malformations diagnosed by ultrasound in the third trimester of pregnancy, without significant harm. The technical aspects of MRI study of the fetus are discussed.

\section{SPACE 1771}

Comparison of dynamic contrast enhanced snapshot flash MR imaging with conventional sequences in the detection and characterization of liver tumors

A. Rahmouni, F. Laurent, M. C. Anglade, B. Houang, P. Gheung, J. M. Bizet, J. Drouillard, D. Mathieu (Presenter); Créteil

Purpose: In order to evaluate the clinical potential of Snapshot Flash MR imaging in liver tumors.

Methods and Materials: 65 patients with liver tumors including: metastasis (18), hepatocellular carcinoma (17), hemangioma (18), FNH (8), and adenoma (4) were imaged using this prospective protocol on a MAGNETOM SP 631.5 $\mathrm{T}$ : T2-weighted images, unenhanced and enhanced Snapshot Flash sequences after bolus IV injection of $0.1 \mathrm{mmol} / \mathrm{kg} \mathrm{Gd}$-DOTA, and finally delayed postcontrast $\mathrm{Tl}$-weighted images.

Results: 1. All the lesions were detected on unenhanced Snapshot Flash MR images, 2. On T2-weighted images no more lesions were depicted than seen on enhanced Snapshot Flash MR sequence, 3. Snapshot Flash imaging is better than T2-weighted images for differentiating hemangioma from hypervascular metastases.

Conclusions: At 1.5 T, despite its low spatial resolution, subsecond dynamic Snapshot Flash MR imaging is a sequence as accurate as spin-echo T2 weighted in detection and characterization of liver tumors.

\section{SPACE 1772}

Proton nuclear magnetic resonance spectroscopy ('H NMRS) of normoxic and ischemic renal tissue

F. Ruiz Santiago (Presenter), J. Fernandez Mena, F. Perán Mesa, A.M. Vargas Morales; Granada

Purpose: 1 . To research on an experimental level by 'H NMRS the effects of ischemia on content of metabolites in rat renal tissue.

2. To discuss about the possible clinical usefulness of these findings and its possible advantages with regard to the other nuclei that have been used for renal spectroscopy investigation, basically ${ }^{31} \mathrm{P}$.

Methods: 40 rat kidneys were used in these experiments: normoxic (10) and ischemic by vascular occlusion for $2^{\prime}(10), 45^{\prime}(10)$ and $60^{\prime}(10)$.

The Kidneys were exposed through a ventral incision, removed and minced in perchloric acid, homogenized and centrifuged. The supernatant was lyophilized and reconstitued in $0^{\prime} 5 \mathrm{ml}$ of $\mathrm{D}_{2} \mathrm{O}$.

NMR spectra were obtained by presaturation using a Brucker AM-300 spectrometer tuned to $297,85 \mathrm{MHz}$ for protons.

Results: 1. We have assigned 41 resonances belonging to 28 different metabolites.

2. We have detected, by effects of ischemia, a significative increase of lactate, glutamate, succinate, glycine and inositol, with regard to normoxic levels.

Conclusions: I. The different content of metabolites between normoxic and ischemic kidney allow to the diagnostic of renal ischemia by 'H NMRS.

2 . With regard ${ }^{31} \mathrm{P}$ NMR, 'H NMR detect a greater number of metabolites, what joined its higher sensibility makes it potentially useful for experimental and clinical research.

\section{SPACE 1773}

Effects of ischemia and hypoxia on renal content of metabolites detected by proton nuclear magnetic resonance spectroscopy ('H NMRS)

F. Ruiz Santiago (Presenter), J. Fernández Mena, F. Perán Mesa, A.M. Vargas Morales; Granada

Purpose: 1 . To research on an experimental level by 'H NMRS the effects of ischemia and hypoxia on content of metabolites in rat renal tissue.

2. Assignation of metabolites responsible for the changes observed in the spectra of tissue extracts and whole kidney.

Methods and Materials: 50 kidneys were used in these experiments: 10 normoxic, 10 hypoxic by asphyxia and 30 ischemic by vascular occlusion for 2 $(10), 45(10)$ and $60(10) \mathrm{min}$.

The kidneys were exposed through a ventral incision, removed and freezeclamped.

NMR spectrum of neutralized perchloric extracts and whole kidneys in phosphate buffer were obtained by presaturation using a Brucker AM-300 spectrometer tuned to $297,85 \mathrm{MHz}$ for protons.

Results: 1. The ischemic injury lead to a significative increase of lactate, glutamate, succinate, glycine and inositol, with regard to normoxic levels. This increase, that begins at 2 min., becomes stable or decreases starting from 45 min. The relationship of these findings with irreversibility of ischemic renal failure was not determined.

2. The persistent renal flow hypoxic injury lead to a significative decrease of glutamate, glycerophosphorylcholine (GPC) and inositol.

3 . In the whole kidney spectra were clearly resolved the resonances of glutamate, succinate, GPC, Glycine and inositol, whereas the lactate resonance is obscured by lipids protons.

\section{SPACE 1774}

Diagnosis of local recurrencles in the small pelvis with dynamic Gd-DTPA enhanced MRI

H. Hötzinger, R. Salbeck (Presenter); Herne

Purpose: The diagnosis of local recurrencies in the small pelvis with conventional diagnostic procedures may be difficult, especially the differentiation between recurrencies and scars. The study presents preliminary results with dynamic MRI after i.v. Gd-DTPA.

Methods and Materials: 12 patients (53-79 years) were examined (15 examinations). There were 7 recurrencies and 5 scars (histologically proven). The primary diagnosis were carcinoma of the rectum (1), urethra (1), endometrium (4), ovary (3) and cervix (3). Contrast medium Gd-DTPA i.v. $2 \mathrm{ml} / 10 \mathrm{~kg} / \mathrm{BW}$. Signal intensities (S1) were determined and measured before and after Gadolinium injection. The differences were calculated in relation to the precontrast value. The signal intensities were measured every minute till 7 minutes. As reference tissues we used fat and muscle.

Results: Recurrencies showed a statistically higher enhancement after the injection of contrast medium in contrary to scars, fat and muscle (table I).

\begin{tabular}{lrrrr}
\hline time (min) & 1 & 2 & 5 & 7 \\
S1 & & & & \\
\hline recurrency & 62 & 86 & 94 & 93 \\
scar & 31 & 34 & 32 & 32 \\
fat & 8 & 7 & 7 & 8 \\
muscle & 18 & 16 & 20 & 18 \\
\hline
\end{tabular}

tab. 1:

S1 after Gd-DTPA i.v.

Conclusions: Dynamic MRI with Gd-DTPA i.v. seems to be a valuable tool for the differentation between local recurrencies and scars in the small pelvis. 


\section{SPACE 1775}

Contribution of MR in evaluation of peridlaphragmatic densities G. Schmutz (Presenter), F.R. Plante, R.K. Chhem, M.J. Jeung, G. Massard, J.M. Wihlm; Sherbrooke; Quebec

Purpose: To determine the contribution of MR Imaging in the evaluation of densities located at the thoraco-abdominal junction.

Material: Retrospectively during three years (April 1987-June 1990) we selected 31 patients with demonstrative densities of peridiaphragmatic region and evaluated by MR Imaging. MRI were obtained in the three planes with a superconducting magnet operating at $0.50 \mathrm{~T}$ using the spin echo technique (T1 weighted and $\mathrm{T} 2$ weighted sequences). The etiologies were: pleural lesions (6 cases), hepatic or right subphrenic diseases ( 4 cases), splenic lesions ( 9 cases), gastric tumor ( 3 cases), hernia (6 cases), pancreatic pseudo-cyst ( 3 cases).

Results: All densities were visualized with MRI. The size of all lesions exceeded $2 \mathrm{~cm}$ in at least one dimension. The connections with diaphragm were always determined. MR Imaging was also helpful in the characterization of the lesions, especially to differentiate solid mass and liquid mass.

Conclusions: MR Imaging appeared contributive in the determination of connections with diaphragm and in the characterization of the peridiaphragmatic densities.

\section{SPACE 1776}

Comparative evaluation of CT and MR imaging in esophageal extramucosal masses

G. Schmutz (Presenter), R. K. Chhem, G. Massard, M.Y. Jeung, J.M. Wihlm, B. Bui; Sherbrooke; Quebec

Purpose: A prospective comparative evaluation of MR Imaging and CT in staging and diagnosis of esophageal extramucosal masses.

Methods: CT Scans and MR Images were obtained in 32 patients with esophageal extramucosal disease detected on barium esophagograms. CT was performed before and after intravenous administration of contrast material. Spin-Echo sequences $\mathrm{T} 1$ weighted and $\mathrm{T} 2$ weighted were obtained on a $0.5 \mathrm{~T}$ superconducting magnet. Surgically proved etiologies were malignant extrinsic involvement (10 cases), leiomyoma (5 cases), lipoma (3 cases), mediastinal abscess ( 3 cases), cystic duplication ( 4 cases), cystic lymphangioma ( 3 cases), pancreatic cyst ( 2 cases) and hydatid cyst (I case).

Results: The size of all esophageal masses exceeded $2 \mathrm{~cm}$ in at least one dimension and all lesions were seen on both CT Scans and MR Images. The CT and MR findings depended on the cause of the lesion. The connections with mediastinal structures were always determined.

Conclusions: MR Imaging and C.T were equally good in delineating the extension of an esophageal lesion and to evaluate the connections with mediastinal structures. However, except in the cases of lipoma, MR Imaging was superior to CT in helping to characterize the lesions, especially those on the distal esophagus where cardiac movements decreased the quality of the CT Scans.

\section{SPACE 1777}

Gd-DTPA-enhanced MRI of degenerative cervical spine disease Peter Schubeus (Presenter), Wolfgang Schörner, Bernhard Sander, Uwe Tosch, Thomas Heim, Roland Felix; Berlin

Purpose: Aim of the present study was to assess the effects and the value of Gd-DTPA administration in MRI of degenerative cervical spine disease. Methods: The study included 5 normal controls and 32 patients with 30 disk herniations and 5 bony stenoses. Examinations were performed with an $1.5 \mathrm{~T}$ Magnetom (Siemens), using a 2D-FLASH sequence (TR $500 \mathrm{~ms}$, TE $5.8 \mathrm{~ms}$ ). Plain T1-weighted (flip angle $70^{\circ}$ ), plain proton density-weighted (flip angle $10^{\circ}$ ) and enhanced $\mathrm{Tl}$-weighted images were obtained. Slice thickness was $2 \mathrm{~mm}$.

Results: Displacement or interruption of the epidural enhancement, nonenhancing structures surrounded by epidural enhancement, and atypical intraforaminal patterns after enhancement were frequent findings in patients, whereas these features were not found in normal controls. The image's diagnostic value was found to be improved after Gd-DTPA administration in about $50 \%$ of the cases, mainly in patients with lateral and intraforaminal disk herniations.

Conclusions: Gd-DTPA may improve diagnosis of degenerative cervical spine disease with MRI, especially in cases of lateral and intraforaminal disk herniations.
SPACE 1778

Invasive cervical carcinoma: assessment of parametrial status with MR imaging

S. Sironi (Presenter), A. Vanzulli, G. L. Taccagni, A. DelMaschio; Milan

Purpose: We conducted a prospective investigation to determine the capability of MR imaging to demonstrate parametrial tumor invasion.

Methods and Materials: 29 patients with histologically proven cervical carcinoma (stage IB or higher) were studied with MR imaging at $0.5 \mathrm{~T}$. All patients underwent detailed histologic evaluation of the parametrium by means of radical hysterectomy. MR imaging findings were compared with microscopic pathologic findings in all cases. T1 weighted (SE 350/25) and T2 weighted (SE 2000/40-90) images in sagittal and axial planes were obtained $(0.5 \mathrm{~cm}=$ slice thickness $)$.

Results: MR imaging showed tumor positive parametrium in 10 patients; in seven of these cases histologic analysis demonstrated parametrial tumor involvement. In the remaining 3 cases no parametrial invasion was found on pathologic examination. MR imaging revealed tumor negative parametrium in 19 cases; in all these patients pathologic study confirmed no evidence of tumor extension into the parametria. The overall accuracy of MR imaging in demonstrating parametrial invasion by tumor was $89 \%$.

Conclusions: Precise knowledge of parametrial tumor extension affects the therapeutic decision between surgery and radiation therapy. The results indicate high MR imaging accuracy in demonstrating parametrial status; therefore MR imaging should be used to determine appropriate therapy in patients with invasive cervical cancer.

\section{SPACE 1779}

Staging and follow up of haematogenous spondylitis by MRI: use of out of phase long TR gradient recalled images

A. Stäbler (Presenter), A. Krödel, D. Widenka, Ch. Gärtner, M. Seiderer, J. Lissner; Munich

Purpose: The value of opposed long TR GE-sequences in staging patients with spondylitis is shown by comparing follow up MR-studies with histology, bacteriology and the clinical course.

Methods: In 47 patients with spondylitis $121 \mathrm{MR}$-studies were performed. 25 patients were studied preoperatively, 3 weeeks, 3 and 6 months postoperatively. Sagittal and transverse $\mathrm{Tl}-\mathrm{w}(\mathrm{TR} / \mathrm{TE}=500 \mathrm{~ms} / 17 \mathrm{~ms})$ and Opposed Long TR Ge images (TR/TE $/ \alpha=500 \mathrm{~ms} / 17 \mathrm{~ms} / 90^{\circ}$ ) were obtained (1.OT, Magnetom, Siemens AG). At corresponding sites we correlated signal intensities of pathologic and healthy bone marrow intraindividually and got the ratio SI (SE) path/SI (GE) path.

Results: In all cases a tumor could be excluded, soft tissue involvement or invasion of the spinal canal could be demonstrated. The SE/GE-ratio can estimate the acuteness of the infection (acute $=$ It 0.7 , subacute $=1$, healing $=$ gt 1.5). Due to bone marrow edema in subacute spondylitis signal loss on T1-w images persists, but in case of susceptibility effects GE-signal returns nearly to normal. Healed spondylitis leads to reactive fatty bone marrow.

Conclusions: Using TI-W SE and opposed long TR GE sequences MRI provides reliable preoperative staging and follow up of spondylitis. There is correlation of SE/GE-ratio with the stage of infection.

\section{SPACE 1780}

MRI follow-up of mediastinal Hodgkin's disease (HD) during and after treatment

J. D. Tesoro-Tess (Presenter), L. Balzarini, E. Ceglia, R. Petrillo, Y. Rayner, R. Musumeci; Milano

1. A persistent mediastinal abnormality after successful treatment for HD is often present and can simulate persistent disease causing diagnostic difficulties.

2. In order to evaluate the possible role of MRI in determining the true nature of these abnormalities, $92 \mathrm{MRI}$ examinations performed in $36 \mathrm{HD}$ patients during and after therapy were reviewed. All patients had proven mediastinal adenopathies at disease presentation and residual mediastinal mass during or after treatment. MRI was performed utilizing SE techniques and both mainly $\mathrm{T} 1$ and $\mathrm{T} 2$ gated images were acquired. MRI results were compared to clinical, radiological and laboratory tests (Gallium 67 ).

3. High signal compatible with persistent or recurrent disease was observed in $26 / 92(28 \%)$ examinations whereas in $44 / 92(48 \%)$ low signal (fibrosis) was achieved. From the comparison with other evaluations, only six false positive (7\%) and I false negative (1\%) MRI results were encountered. The overall accuracy of MRI was $92 \%$, sensitivity $96 \%$ and specificity $90 \%$.

A correlation MRI/Ga 67 scan was available in $39 / 92$ examinations $(42 \%)$ and $7(18 \%)$ discordant reports were encountered, mainly due to positive MRI with negative Ga67. 


\section{SPACE 178}

${ }^{31} \mathrm{P}$ magnetic resonance for assessment of the viability of human transplant kidneys, preserved with three different preservation solutions A. van Die (Presenter), J. van der Grond, R. J. Hené, W. P. Th. M. Mali; Utrecht

Purpose: In vivo ${ }^{31} \mathrm{P}$ MR spectroscopy is nowadays commonly used to investigate, non invasively, the metabolic state or viability of several organs. ${ }^{31} \mathrm{P} M R$ spectroscopy also could obtain information about the viability of transplant kidneys, and the effect on renal viability, when using different preservation solutions.

Methods: ${ }^{31} \mathrm{P}$ MR spectroscopy was performed on twenty human transplant kidneys, 2 hours before transplantation, 1 till 24 hours after explantation. Six kidneys were preserved in Euro Collins (EC) solution, six kidneys were preserved in University of Wisconsin (UW) solution, whereas eight kidneys were preserved in HTK solution. Image guided, volume selective ${ }^{31} \mathrm{P}$ MR spectroscopy, at $0^{\circ} \mathrm{C}$, was performed, using a $1.5 \mathrm{~T}$ whole body spectrometer. Results and Conclusions: In the kidneys who were preserved in UW and EC, large phosphomonoester (PM) and inorganic phosphate ( $\mathrm{Pi}$ ) peaks could be observed. However, both peaks did not show any significant correlation both with the time after explantation and renal viability after transplantation. The ${ }^{31} \mathrm{P}$ MR spectra of kidneys who were preserved in HTK did show in addition to PM and Pi resonances also adenosine triphosphate (ATP) and phosphodiester (PD) resonances. The intensity of these latter peaks showed a positive correlation with the viability of the transplant kidney, which was approved by renal viability after transplantation.

Since renal ATP and PD peaks are absent when kidneys are preserved in UW or EC solution, ${ }^{31} \mathrm{P}$ MR spectroscopy is not capable to predict renal viability after transplantation when kidneys are preserved in one of these solutions. However, when kidneys are preserved in HTK, ${ }^{31} \mathrm{P}$ MR spectroscopy is a non invasive technique to predict renal viability before transplantation.

\section{SPACE 1782}

Early cervical carcinoma (FIGO stage IA): diagnostic accuracy of MRI A. Vanzulli (Presenter), S. Sironi, C. Belloni, G. L. Taccagni, A. DelMaschio; Milan

Purpose: Cervical carcinoma is classified by FIGO criteria as stage IA when the stromal invasion is limited to $5 \mathrm{~mm}$ or less and as stage IB when the invasion is more than $5 \mathrm{~mm}$. Conization is possible in stage IA; hysterectomy is mandatory in stage IB. Purpose of this study is the evaluation of 0.5 T MRI sensitivity in staging early cervical carcinoma.

Methods: 12 patients with clinically small uterine cervical carcinoma have been studied with $0.5 \mathrm{~T} \mathrm{MRI}$; the surgical specimens were evaluated (FIGO criteria).

Results: Pathologic examination indicated stage IA carcinoma in 5 patients and stage IB in 7 patients. MRI correctly staged 2 patients with IA cancer $(40 \%)$ and 6 patients with IB disease $(85.7 \%) .3$ patients were overstaged and I patient understaged. The pathologic examination of the specimens of the patients who were overstaged showed the presence of dilated cervical glands that mimicked neoplasia in T2W sequences. In the evaluation of stage IA/IB uterine cervical cancer MRI had sensitivity $=85 \%$, specificity $=40 \%$, positive predictive value $=66 \%$ and negative predictive value $=40 \%$.

Conclusions: Low specificity and low negative predictive value limit the usefulness of MRI in the evaluation of uterine cervical carcinoma FIGO stage 1.

\section{SPACE 1783}

MRI characterization of hydatid cysts

Walther von Sinner (Presenter), Lambertus te Strake; Riyadh

Twenty (20) patients with hydatid disease were examined (liver/abdomen $\mathbf{n}=$ 12 , lungs/chest $\mathbf{n}=3$, heart $\mathbf{n}=3$, brain $n=1$, bone $\mathbf{n}=1$ ). The purpose of this exhibit is to show different MRI manifestations and characteristics of hydatid disease. Fourteen (14) examinations were performed on a $1.5 \mathrm{~T}$ scanner using short TR/TE and long TR/TE 2000/100 conventional SE pulse sequences. Slice thickness $=8 \mathrm{~mm}$. NEX $=4$ (short TR $/$ TE sequence) and 2 (long TR/TE sequence). Six (6) examinations were performed on a $0.5 \mathrm{~T}$ scanner.

Results: 1 . The excellent contrast resolution renders MRI the best single test to study both cyst contents and reactive changes of the host tissue.

2 . The characteristic but non-specific low intensity wall of the intact cyst represents both the endocyst (parasitic membranes) and the pericyst (host reaction). 3. Degenerative changes of the cyst wall and subsequent rupture can be directly visualized on MRI.

4. The multiplanar imaging capability of MRI allows superior mapping of the spread of hydatid disease.

5. MRI is an excellent method to monitor the full effects of albendazole treatment on both the cyst and host tissue.
6. Despite the fact that calcifications are better seen on CT, MRI may be considered the method of choice to image the spectrum of hydatid disease.

\section{SPACE 1784}

Three dimensional MR angiography with $0.3 \mathrm{~T}$ vertical field MRI Hiroshi Nishimura, Shigeru Watanabe (Presenter), Shinji Kawasaki; Chiba

Purpose: Our main purpose is to evaluate MR angiography for making effective visualization of vascular structure with $0.3 \mathrm{~T}$ vertical field MRI through comparison of 3 dimensional phase sensitive and sequential acquisitions of 2 dimensional inflow in 3 dimensional display.

Methods: We have developed both 3 dimensional phase sensitive MR angiography obtained by sequence subtraction of dephasing gradient echo from rephasing (velocity-compensated) one, and 2 dimensional sequential inflow by rephasing gradient echo. MR angiograms were obtained with a ray tracing of the new modified maximum intensity projection at various viewing angles. These images are displayed on CRT in high speed cyclic mode to depict the front-rear relation of vessels for recognition.

Results: Using Eddy-current-free magnet system, 3 dimensional phase sensitive MRA could depict crisp intracranial vascular structure and there was improved delineation of middle cerebral artery branches and arterior cerebral arteries at low flip angle. Sequential $2 \mathrm{D}$ inflow revealed effective for cervical and abdominal vascular structures.

Conclusions: We could obtain an excellent detectability of vascular structures by both $3 \mathrm{D}$ phase sensitive and $2 \mathrm{D}$ inflow even with $0.3 \mathrm{~T}$ vertical field MRI. Arteries and veins could be differentiated well with presaturation of upperstream.

\section{SPACE 1785}

Assessment of cerebral blood flow with dynamic contrast-enhanced T2* weighted gradient echo images

Klaus U. Wentz (Presenter), F. Gückel, G. Brix, J. Röther, W. Jaschke, R. Loose, M. Deimling, M. Georgi; Mannheim

Local magnetic field inhomogeneities induced by paramagnetic contrast agents applied as a bolus lead to a transient signal loss in $\mathrm{T} 2 *$ weighted gradient echo images while the bolus is passing through the tissue. This loss of signal intensity is correlated to the regional tissue perfusion.

In our prospective study 30 patients (15 with no cerebrovascular disease, 15 with vascular stenosis brain infarctions or cerebral neoplasms) were examined. The goal of our study was to examine whether this method leads to clinically relevant results concerning local brain perfusion. We have applied a series of fast T2* weighted gradient echo images $(2.9 \mathrm{sec} /$ image) during and after bolus application of GdDTPA. From this series of images the local changes in the signal intensity were computed pixel by pixel. From these data a perfusion image was calculated. These perfusion images show significant perfusion differences between white and grey matter. Regions of cerebral infarctions can be clearly identified as regions with lower perfusion. This method provides information about cerebral blood flow dynamics not available from conventional MR imaging.

\section{SE PACS (12) \\ SPACE 1786}

\section{Calibration of laser printers to gray scale monitors}

Samuel J. Dwyer III, A. W. Templeton, Larry T. Cook (Presenter), Edward L. Siegel; Kansas City, KS

Purpose: Faithful film hardcopy reproduction by laser film printers of images displayed on high-resolution gray-scale workstations is diagnostically mandatory. This exhibit demonstrates a technique for accomplishing faithful reproduction on laser film printers.

Methods and Materials: A gray-scale controller has been developed by our Department. The controller samples the viewing screen of the gray-scale monitor. This sample is processed and the results are used to automatically select the optimum lookup table for faithfully reproducing the displayed images. The gray-scale controller has been evaluated for CT, MRI, and storage phosphor plate images.

Results: An evaluation of the gray-scale controller demonstrates that with careful selection of the display's assigned gray levels and with proper modification of the laser printer's lookup tables, the laser printed images faithfully reproduce the displayed images. The gray scale display is driven by 8 bit digital-to-analog converters. The laser film printer generates 12 bit pixel values. Our gray-scale controller enables the laser film printer to faithfully reproduce images presented on the gray scale monitors. 


\section{SPACE 1787}

Personal computer as a low-cost Image workstation of MRI Sören Bondestam, Veli-Pekka Poutanen, Carl-Gustaf StandertskjöldNordenstam (Presenter); Helsinki

Purpose: The aim of the project was to retrieve MRI images from Siemens Magnetom I T to an Apple Macintosh IIci using commercial programs without using expertise or programming.

Methods and Materials: A Macintosh IIci with 8 bit display was linked to the Digital Micro Vax II of the Magnetom using Ethernet. The terminal program was a freely distributed demo from Thursby Software Systems. Inc (TSSnet $v$. 2.0). Images were converted and displayed using Explorer v. 0.9b (UCLA). The uncompiled version of the program was decoded using THINK C.

Results: The images were copied to the Macintosh within 5 seconds per image. The source code of the Explorer program's pict I/O file included a 12 bit image conversion, which was activated instead of the 8 bit routine. Explorer enables ROI functions with histogram and saving. The savings are readily opened in spreadsheet or statistical programs for further analysis. Image mathematics are easily done, i.e. subtraction. Compared to dedicated workstations this system was very cost effective. Personal computer costs: hardware $\$ \approx 5300$, software $\$ 263$.

\section{SPACE 1788}

Dial-up Teleradiology system: evaluation of components and clinical experiences

A.W. Templeton (Presenter), S. J. Dwyer III, D. A. Eckard, L. A. Harrison, J. R. Bergh; Kansas City, KS

Purpose: Teleradiology systems provide for radiographic service to remote sites and under served areas. Our Department has implemented and is operating a wide area network with dial-up communications for transmitting radiologic image data. Two army base sites are operational on the Teleradiology system. This exhibit analyzes our teleradiology system components and our clinical experiences.

Methods and Materials: Our teleradiology system uses fiber optics for the long-haul carrier portion of the wide area network. The local carrier portion of the connection into the two Army hospitals is a mixture of fiber optic cables and coaxial wire cables. The transmission service is a dial-up, selectable multiple of switched $56 \mathrm{k}$ bit/second links $(\mathrm{N} \times 56 \mathrm{k}$ bit/second lines). The user selects the data rate to be used and is charged only for usage time. Data transmission is parallel over the switched $56 \mathrm{k}$ bit/second lines at $\$ 0.06 \mathrm{per}$ minute per switched line. A laser film digitizer is used at each site and a laser film printer and an interactive gray scale display is used at our Department. Results: The transmitted $2 \mathrm{~K} \times 2 \mathrm{~K} \times 12$ bit radiologic images are all very diagnostic. An evaluation of six months of operation will be presented.

\section{SE}

\section{Education (14)}

\section{SPACE $\underline{1789}$}

\section{Sonographic imago artifacts}

Beatrice L. Madrazo (Presenter), Ali Shirkhoda, Lance Hefner; Royal Oak, MI

Imaging methods are fraught with artifacts that are frequently seen within the field of view. In diagnostic US, artifact can simulare disease, and therefore their recognition is essential, to avoid misdiagnosis. This exhibit will be illustrated with a variety of image artifacts, such as reverberations, duplication mirror images, comet tail, acoustic shadowing, acoustic enhancement, refraction, side lobes, slice thickness, and tissue velocity. Doppler artifacts, for both duplex and color Doppler techniques, such as aliasing, tissue vibrations and mirror images, will also be included. Each artifact will be illustrated with clinical examples as well as an explanation of the underlying physical principles.

\section{SE}

\section{SPACE 1790}

Omnipaque filled in plastic bottles - pharmaceutical development Lisbeth Aanstad (Presenter), Bodil Weng; Oslo

The purpose of this work was to develop a plastic bottle of $50 \mathrm{ml}$ for Nycomed's X-ray contrast media Omnipaque 300 and $350 \mathrm{mgl} / \mathrm{ml}$.

To achieve a high quality sterile plastic bottle, we decided to choose an aseptic blow, fill and seal method, the Bottelpack ${ }^{\oplus}$ method, developed by Rommelag AG in Switzerland.

To ensure an autoclaved sterile bottle we decided to use polypropylene as plastic material. Polypropylene is an inert plastic material, practically free from additives.

Migration tests have been performed to ensure that no extractables migrate from the plastic material into the contrast media.

The product has been tested on toxicity, both initially and after storage at elevated temperature. Thermal stability tests have been performed to ensure a long shelf life of the product.

One of the main items of our development work was to achieve a user-friendly bottle with a design corresponding to the users demands. To get information about the demands we performed a marked research in France, England and Sweden.

A survey of the production method, the conclusions from the market research and the results from the stability study will be presented in the poster.

\section{SPACE 1791}

3D-reconstruction and volume measurement of operation defects deformations of the ventricles by $2 D$-magnetic resonance tomography K. Bootsveld (Presenter), B. Siewert, F. Träber, W. A. Kaiser, M. Reiser, C. E. Elger; Bonn

Purpose: For the evaluation of operation defects due to surgical treatment of epilepsy an exact localisation and measurement of the volume of the defects is necessary.

Furthermore, changes of the ventricle shape depending on time and therapyfollow up must be exactly analyzed.

Methods: 2D-Gradient-Echo-Sequences (Fast Field Echo; TR $2000 \mathrm{~ms}$, TE $45 \mathrm{~ms}$, Flip angle 90 degrees) and 2D-Spinecho-Sequences (TR $3000 \mathrm{~ms}$, TE $20,90 \mathrm{~ms}$ ) were applied in a slice thickness below $3 \mathrm{~mm}$ in the investigation of 31 patients (22 surgical treatment of epilepsy, 9 congenital or acquired deformations of the ventricles). The reconstruction of these thin slices allowed a 3D-presentation of the brain or ventricles.

Results: The reconstructed images allow an exact volumetric measurement of the defects and the ventricles and a precise delineation of the surgical resection borders.

Conclusions: The reconstructed 3D-images allow the exact analysis of operation defects and malformations of the ventricles and their relation to other brain structures. E.g. in the interpretation of the surgical treatment of temporal lobe epilepsy this method can be recommended.

\section{SPACE 1792}

\section{CT reformation software programs in clinical practice}

F. Carini (Presenter), D. Intropido, E. Esposti, V. Pallavera, G. Porchera; Lodi

We report our experience about the use of Computerized Tomography (CT) imaging reformation programs in the clinical practice.

Especially we emphasise the possibility to correct the image according to anatomical axes that is normally impossible with simple CT axial scans.

We considered especially the application of continuous reformation that uses a single reference reformation image to create single or multiple reformation images along parallel planes to correct the parallelism between axial plane and intervertebral disk space in accentuated scoliosis or lumbar hyper-lordosis with optimum reconstruction of spaces and better visualization of herniated disk.

The same program proved to be valuable in reconstructing from a regular axial scan sequence of the skull the complete sequence with correct inclination for temperal lobe without excessive image degradation.

We also studied the application of oblique reformation software that uses two member images to create a single or a multiple oblique image reformatted along parallel plaines. The program proved to be useful in the bronchial tree examination with better stenosis visualization and improved spatial appreciation of peri-bronchial lesions.

In conclusion modern CT reformation software programs with low or absent image degradation enable the use of plaines different from the normal axial CT plaines with anatomical angles correction and better lesion visualization.

\section{SPACE 1793}

Digital fluorography versus conventional radiography: comparison of diagnosis efficiency, film saving and radiation exposure

J. Drouillard (Presenter), F. Laurent, V. Lafarge, G. de Verbizier, B. Houang, B. Chaulin; Bordeaux

The aim of the study was to compare image quality, film consumption and delivered radiation dose between digital spot imaging (DSI) and conventional cassette films.

A prospective randomized study of 180 patients was conducted comparing DSI studies (92) and cassette films (88) obtained from the same remotecontrolled X-ray stand and a 14, 10,6.5 in image intensifier. A strict protocol was followed in gastro-intestinal studies (76), venographies (32), lumbar myelograms (10) and genito-urinary studies (72). Criteria of evaluation included diagnostic efficiency, film saving and delivered radiation dose. 
In all cases imaging quality was sufficient for the diagnosis. The gain in film cost with DSI varied from $30 \%$ (hysterographies) to $72 \%$ (IVU). With DSI the delivered radiation dose was lower and the gain varied from $32 \%$ for small bowel examinations to $66 \%$ for IVU and $69 \%$ for esophagograms including swallowing evaluation.

The experience obtained over two years of working with digital fluorography and our randomized study had led us to confirm the diagnosis efficiency, financial economy and reduction in radiation exposure obtainable with the DSI system.

\section{SPACE 1794}

Three-dimensional mapping of lithotripsy shock waves

Timothy J. Hall (Presenter), Michael F. Insana, Nannette M. Soller, Stanton J. Rosenthal, Samuel J. Dwyer III.; Kansas City, KS

Purpose: A previous study has shown that the presence of a rippled fat-mimicking layer significantly distorts the shock wave pressure distribution in the focal plane of an extracorporeal shock wave lithotripter.

Methods and Materials: The use of a novel, very durable hydrophone will allow mapping the pressure distribution in three dimensions. A three dimensional pressure map will give a more complete description of the effects of wave front distortion (phase aberration) on stone breakage and tissue damage in ESWL treatments.

Results: Comparisons of pressure maps both with and without aberrating layers will be included. Performance characteristics of this hydrophone will also be described.

\section{SPACE 1795}

Diagnosis of fractures using the digital storage phosphor system H. M. Klein (Presenter), B. Wein, A. Stargardt, R. W. Günther; Aachen

Conventional screen-film radiographs were compared with storage phosphor images concerning diagnostic performance in traumatologic radiography. We used an image pool of 106 radiographs including 50 conventionally and 56 digitally produced images. The images were reviewed by four experienced radiologists. Detectability of fractures was coded in a five-point scale of confidence and analysed by ROC-statistics. Furthermore the image quality and the optical density were compared.

Digital images are superior under difficult exposure conditions and offer advantages by additional image processing and documentation. For some indications (follow-up, functional examinations), reduced x-ray exposure is tolerable. The detectability of subtle lesions, which is essential for the primary diagnosis of fractures was significantly better by conventional radiography.

\section{SPACE 1796}

Clinical evaluation of a $100-\mathrm{mm}$ film digitizer and image processor for film archiving

Michio Kono (Presenter), Katsuhito Yamasaki, Eiichiro Itouji, Shuji Adachi, Masahiko Kusumoto, Eiro Sakai; Kobe

Purpose: The Japanese law recommended the use of $100-\mathrm{mm}$ roll-film fluoroscopic radiograph for mass screening. Therefore we developed a roll-film digitizer and image processor and evaluated its effectiveness.

Methods and Materials: This system is a prototype roll-film image digitizing system (Hitachi Medical Corporation) that consists of a laser scan digitizer, a high-speed image processing unit, and an optical disk drive or optical disk library. The contrast and spatial resolution of digitized 100-mm fluoroscopic radiographs displayed on a CRT were evaluated experimentally with use of a Burger phantom and a Hewlett chart. Modulation transfer function characteristics were studied to assess the quality of the CRT Image.

Results: This system was used for routine mass screening of 5.000 cases for lung cancer. These 100-mm fluoroscopic radiographs of the chest from mass screening for lung cancer were digitized with a $100-\mu \mathrm{m}$ pixel size and 12-bit gray-scale resolution. Radiologists evaluated the digitized images as displayed on a CRT. The diagnostic accuracy of this system was compared with that of conventional $100-\mathrm{mm}$ fluoroscopic radiographs and was found to be better. Conclusions: The $100-\mathrm{mm}$ roll-film digitizer and the image processor are useful as a miniature picture archiving and communication system for the mass screening of patients with lung cancer.

\section{SPACE $\underline{1797}$}

Phosphor plate imaging: exposure reduction

Norman L. Martin (Presenter), Larry T. Cook, Samuel J. Dwyer III., Michael F. Insana, A. W. Templeton, Timothy J. Hall; Kansas City, KS

Purpose: The diagnostic accuracy of a photostimulable phosphor imaging plate system with interactive workstation was studied using a 25 percent and 50 percent exposure reduction.

Methods and Materials: Dose reduction was accomplished using constant kVp and a 25 percent and 50 percent decrease in $\mathrm{mAs}$. Anatomical phantoms, thirty conventional and phosphor plate, and manipulated chest, cervical spine, breast, and extremity examinations have been compared. Digital plate images were archived on an optical disc and displayed/manipulated on the interactive workstation. Laser printed film images were generated from both the digital plates and the workstation.

Results: This study demonstrates equivalent results for 25 percent and 50 percent dose reduction. The impact of dose reduction on image manipulation and quality is presented.

\section{SPACE 1798}

Sonographic diagnosis of hepatosplenic tuberculosis G. Pia (Presenter), L. Pascalis, G. Aresu; Cagliari

30 patients (20 females and 10 males) between 25 and 50 years of age with hepatic and/or splenic localization of tubercular disease, were studied with ultrasonography. Previously a form of pulmonary tuberculosis had been diagnosed in these patients. All of them were subjected to CT scan: 5 to hepatic angiography and 20 to percutaneous biopsy of the liver under ultrasound guidance. In 25 subjects ultrasonography evidenced a nodular formation restricted to the liver whereas in the other 5 patients, besides at hepatic level, lesions were also present in the spleen. These formations were roundish with a diameter varying from 5 to $7 \mathrm{~cm}$ and presented a hyperechoic central area and a peripheral hypoechoic halo. Liver biopsy of the lesions under ultrasound guidance made it possible to evidence fact of inflammatory cells and more specific epithelioid cells in caseous necrotizing areas. Ziehl coloration revealed the presence of alcohol acid resistant bacilli. Treatment was started with Ethambutol $800 \mathrm{mg} /$ day, Pyridoxine $500 \mathrm{mg} /$ day and $\mathrm{INH} 200 \mathrm{mg} /$ day and was protracted from a minimum of 6 months to a maximum of 10 months. Patients rapidly improved and after a period of 5 to 9 months from the beginning of treatment, the ultrasonic and tomographic values returned to normal. Our results allow us to affirm that the hepatosplenic localization of tubercular disease is all but rare and must therefore be considered in differential diagnosis of space occupying lesions, at a hepatic as well as at renal levels, in the same way as other pathologies such as neoplasia, parasitic cysts etc.. According to our data it is also possible to affirm that with adequate medical therapy the prognosis of these patients is good.

\section{SPACE 1799}

\section{Vocal input computer assisted reporting}

Gianni Saguatti (Presenter), Marco Miceli; Bologna

Vocal input computer assisted reporting provides immediately to draft, verify and deliver a radiological report.

In the Radiology Department of Maggiore Hospital in Bologna a system with different items has been tested: PC Philips 386; $12 \mathrm{Mb}$ RAM memory: $160 \mathrm{Mb}$ hard disk; VGA colour monitor; board; $1.2 \mathrm{Mb}, 5^{\prime \prime}$ drive; mathematical co-processor; Kurzweil vocal digitization file; Kurzweil microphone; Kurzweil software application; 136 columns, 280 cps printer; MS-DOS software.

The system provides a real time answer, 15000 words vocabulary, 15 minutes user's training, structured phrases utilization and free text interpolation, statistical elaboration possibilities on memorized reports.

Reviewing 2000 traditional draft reports a $\mathbf{4 0 0 0}$ words vocabulary has been composed. However 6-7000 words are considered enough to satisfy all radiological reporting need.

At present this system has not more than $5 \%$ margin of mistake, which is considered fully acceptable.

Moreover the system will soon be provided with RIS and PACS interface.

\section{SE}

\section{SPACE 1800}

Novel endogenous $\alpha$-particle radiotherapy for undifferentiated thyroid cancer

I. Brown (Presenter); Cambridge

Undifferentiated thyroid cancer has a poor prognosis; regardless of therapy, median survival barely exceeds 6 months. Such tumours rarely concentrate radioiodine to an extent sufficient for therapeutic application. Astatine (At), the highest radiohalogen, though more amphoteric than iodine, exhibits some similar physio-chemical properties; it concentrates in normal thyroid tissue to a significant degree. Though the in vivo metabolism of astatine in thyroid tissue is not entirely dissimilar from that of iodide, a precise intracellular biochemical mechanism has not yet been fully elucidated. ${ }^{211}$ At is most suited for therapeutic purposes as it decays by $\alpha$-emissions of mean LET near optimal for radiotherapeutic effects. In order to exploit both its unique biochemical behaviour and nuclear properties, ${ }^{211} \mathrm{At}$ has been investigated as a potential 
$\alpha$-endoradiotherapeutic agent in a human thyroid tumour xenograft model in nude mice.

Biodistribution and $\alpha$-autoradiographic studies were carried out in euthyroid male MFI nu/nu mice, each bearing a single s.c. implanted human undifferentiated thyroid carcinoma. Mice received a single i.v. injection of $370-740 \mathrm{kBq}$ $\mathrm{Na}^{211} \mathrm{At}$ and were sacrificed at intervals over $48 \mathrm{~h}$.

The uptake of ${ }^{211}$ At into thyroid carcinoma xenografts was rapid, and sustained. A significant degree of 211 At localization (11-28\% injected dose ${ }^{211} \mathrm{At} / \mathrm{g}$ tumour) was achieved in $6 / 8$ anaplastic and $2 / 2$ undifferentiated follicular thyroid carcinomata. 211At was found to be homogeneously distributed within malignant cellular areas, with few $\alpha$-tracks originating from stroma. None of these tumours concentrated radioiodine. Radiodosimetric calculations suggest that a tumoricidal high-LET quality radiation dose can be delivered to such tumours by endogeneously targeted ${ }^{21}$ At.

\section{SPACE 1801}

Non-invasive control of the hyperthermia of tumors xenotransplanted into nude mice

G. Bruggmoser (Presenter), W. Hinkelbein, R. Saum; Freiburg

a) Development and testing of a 4-channel-hyperthermia-system for the treatment of human tumors xenotransplanted into nude mice.

b) The tumors are heated by microwaves at $2.45 \mathrm{GHz}$. The treatment is controlled by a microwave radiometer at $3 \mathrm{GHz}$. The whole system is steered by a computer with individually adapted software.

c) By phantom-measurements and in-vivo hyperthermia we could demonstrate, that the treatments can be monitored reliably by non-invasive measurements.

d) Based on this development the effect of hyperthermia to different tumors, dependent on the temperature and the fractionation scheme can be evaluated.

\section{SPACE 1802}

Use of perioperative or split course interstitial brachytherapy techniques for salvage irradiation of Isolated local recurrences (ILR) after conservative management of breast cancer

P. Brunel (Presenter), C. Rozec, C. Maulard, L. Ucla, S. Delanian, J. Huart, M. Housset, F. Baillet; Paris

Treatment of ILR after conservative management for breast cancer is mastectomy. When surgery is contra-indicated or refused brachytherapy may be an alternative. From $01 / 1979$ to $06 / 1990$, we treated 40 pts with ILR of 0,5 to $8,5 \mathrm{~cm}$ (mean $\mathrm{T}=3,5 \mathrm{~cm}$ ), 13 were distant from the primary tumor site. Four ILR (mean $T=4 \mathrm{~cm}$ ) were treated by classical interstitial brachytherapy (CIB) to $60 \mathrm{~Gy}, 11 \mathrm{ILR}$ (mean $\mathrm{T}=2,5 \mathrm{~cm}$ ) by tumorectomy and perioperative interstitial implants (PIB) of 30-35 Gy, 25 ILR LA (mean T $=4 \mathrm{~cm}$ ) by split course implants (SCIB) of 35 and 30 Gy at a 1 month interval with a source shift. Complete tumor regression was obtained in all patients. Eight $(20 \%) \mathrm{pts}$ developed second LR: (1/4 CIB, 3/11 32\% PIB, 4/25 16\% SCIB), 7 had associated distant metastases. $3 / 4$ mastectomies performed after treatment revealed no tumor. The overall 5 years survival was $69 \%$. Two patients developed local complications; skin necrosis which was associated with recurrent tumor in 1 case. Cosmetic results in controlled patients were: no trace $=4$, acceptable sequelae $=9$, unaesthetic results $=14$ cases, unknown $=3$. Interstitial brachytherapy may occasionally be used as alternative therapy for ILR. PIB and SCIB are 2 novel and distinct techniques. Their specific selected indications depend on the size of the tumor. Techniques will be presented.

\section{SPACE 1803}

\section{Nucleosides as markers in patients with cancer}

\section{A. El-Sharkawi (Presenter), C. A. Wellington; Swansea}

Patients with cancer are known to excrete high level of nucleosides in their urine. Measurement of these nucleosides may offer the possibility of not only diagnosing the presence of cancer, but also assessing the response to treatment. We have measured (by high performance liquid chromatography) (HPLC) the nucleosides pseudouridine $(\psi)$, Creatinine $(\mathrm{Cr})$, Cytidine (C), 1-Methyladenosine $\left(m^{\prime} A\right)$, 1-Methylguanosine $\left(m^{\prime} G\right)$, Uridine $(U r)$ in the urine of 19 volunteers assumed to be free of tumours and 20 patients with cancer before and after their radiotherapy treatment.

Mean $\left( \pm\right.$ SD) concentrations $\left(\mathrm{n} \mathrm{mol} \mathrm{cm} \cdot \mathrm{cm}^{-3}\right)$ in the first group found to be $(\Psi)$ $2.8 \pm 2.2$; (Cr) $5.1 \pm 4.6$; (C) $3.3 \pm 2.2$. The other three nucleosides $m^{1} \mathrm{~A}, \mathrm{~m}^{1} \mathrm{G}$ and $U r$ were not detectable. The mean $( \pm S D)$ total nucleosides concentration in the volunteers was $11.2 \pm 5.9 \mathrm{n} \mathrm{mol} \mathrm{cm}^{-3}$. The mean $( \pm \mathrm{SD})$ in the second group before their radiotherapy treatment were found to be $(\psi) 11.5 \pm 9.1$; (Cr) $9.2 \pm 3.1$; (C) $10.9 \pm 18.0$. The mean ( \pm SD) total nucleosides concentration in patients with cancer was $44.8 \pm 29.5$ before treatment, and $10.5 \pm 4.7$ after their radiotherapy treatment.

The results show higher concentration of nucleosides in urine of patients with cancer, and its reduction after radiotherapy treatment to the level of concentration in the volunteer groups who are assumed to be free of tumours. We feel that measurement of urinary nucleosides by High Performance Liquid Chromatography (HPLC) is a very promising non invasive test for cancer diagnosis and monitoring therapy.

\section{SPACE 1804}

Radiotherapy planning for suprasellar tumors in children and young aduits

I. Golubičić (Presenter), Lj. Gržetić, O. Frim, M.Durbaba, V. Šobić; Beograd

Since 1982 we have been planning radiotherapy for suprasellar tumors from three fields. Treatment volume is defined according to the computerised tomography with computerised planning system.

Appropriate isodose distribution being obtained by the use of three fields: two wedged opposite lateral fields and one unwedged frontal field. All patients were irradiated from the Linear Accelerator with photon beam $10 \mathrm{MeV}$. Tumor dose was 50-60 Gy, dose per fraction 1,6-1,8 Gy, five days per week, over 6-8 weeks.

By that approach we treated 25 children and young adults suffered from primary brain tumors with suprasellar localisation (from 2-22 years). All patients had had previous surgery (biopsy or partial removal). 24/25 were histologically proven.

With a follow-up after radiotherapy of 1-9 years all, but two patients are alive (one died from the tumor extension and one from an other cause of death). Assess of tumor response to radiotherapy was based on CT scanning and ophthalmology examination. Follow-up assessements include monitoring of vision, neurological status, endocrine status and school performance, and quality of life of longterm survivors. We will be presenting late complications which have been detected in our group of patients.

\section{SPACE 1805}

Dosimetry of irradiated cultured cell lines using ESR spin trap Mitsuoki Hashiba (Presenter), Hiroyuki Matsuno, Norimoto Tanaka, Kohichi Murakami; Kyoto

Purpose: Detection of free radicals of irradiated cultured cell lines is performed by ESR (EPR) spin trapping method. The conventional measuring technique of ESR spectroscopy has required extremely low temperature of liquid nitrogen level though the biological environment requires room temperature. The purpose of this study is quantitative evaluation of free radicals formed by gamma ray irradiation at room temperature and to observe the relation between ESR spectra and the biological response in several graded doses. Furthermore behavior of SOD (superoxide dismutase) was attempted to confirm in both ESR spectra and biological response.

Methods: PBN (phenyl t-butyl nitrone) was used as ESR spin trap for the stability after irradiation and for the solubility to organic solvent chloroform/methanol made effective to observe ESR spin adducts in room temperature.

Results: The concentration of PBN spin adducts formed by irradiation appeared to be directly proportional to each dose. Adding SOD, the amplitudes of ESR spectra decreased in consequence to irradiation dose.

Conclusions: Quantitative evaluation of free radical formed by irradiation using ESR trapping was examined and the dosimetric usage was confirmed.

\section{SPACE 1806}

Treatment planning for ophthalmic tumours with $1-125$ seeds J. Heikkonen (Presenter), H. Toivola, M. Forss, A. Tarkkanen; Helsinki

The low energy radioactive $I-125$ seeds are ideal for treatment of intraocular tumours. The energy $(28-35 \mathrm{keV})$ is suitable to irradiate tumours as high as $10 \mathrm{~mm}$. The plaque is made from gold sheet about $0.5 \mathrm{~mm}$ thick, completely shielding the extraocular tissues located behind it. The eye applicators were made in pairs, so that a nonradioactive silver plaque can be used for suture positioning. The radioactive $\mathrm{I}-125$ seeds are attached to the gold plaque with silicone rubber caulk (RTV 3140). The I-125 seed plaques can be individually designed for each tumour, depending on its size, shape and location. The depth dose curves and three-dimensional dose distribution in $\mathrm{Gy} / \mathrm{h}$ units are calculated with a computer program based on the polynomical fitting of the measured data. The dose planning can be plotted at any plane desired. The developed program can be applied to optimize the plaque design, I-125 seed positions and the treatment planning. The method is in routine use at our department. 


\section{SPACE 1807}

Clinical application of MR-tomography and MR-angiography in subdiaphragmatic treatment planning of malignant lymphoma

A. Köster (Presenter), B. Kimmig, M. Müller-Schimpfle, G. Layer, G. Brix, W. Semmler, M. Wannenmacher; Heidelberg

Subdiaphragmatic radiation therapy in malignant lymphomas usually include the paraaortic and parailiacal region, the splenic pedicle, the spleen and rarely the liver hilus and prominent lymphatic masses in other locations. Organs with low radiation tolerance like kidneys have to be protected. Purpose of treatment planning is to define an individual and reproductible target volume. We developed and employed a technique to visualize simultaneously abdominal organ and blood vessel anatomy in frontal view by using MRT and MRA.

A computer program was created to superimpose an optimal coronar MR-Tomogram and MR-Angiogram. By using a subtraskope the MRT/MRA-superposition can exactly be projected on a simulation film in correct scale. This novel method was evaluated in 15 patients. The individual target volume could exactly be defined and thereby individual field corrections were possible. Especially significant protection of renal parenchyma was achieved. Treatment planning on simulation film using a subtraskope is simple and can be performed within a mean of 10 minutes. We assume that renal contrast examination, CT-planning and i.v.-DSA can be replaced by this method.

The presented technique is an accurately non-invasive method to optimize and individualize treatment planning in subdiaphragmatic lymphomas.

\section{SPACE $\underline{1808}$}

Antitumor effects of thermosensitive liposome-encapsulated cisplatin T. Nishita (Presenter), T. Nakajima, T. Tada, M. Tsumura, M. Nakanishi, H. Fukuda, M. Sakashita, Y. Onoyama; Osaka City

Purpose: The combined therapy of thermosensitive liposome and local hyperthermia is thought to enhance the anti-tumor effects. TAC-1043 (Takeda Co.) is the thermosensitive liposome that releases entrapped cis-diamminedichloroplatinum (CDDP) above $41^{\circ} \mathrm{C}$. We studied the anti-tumor effects and concentrations of TAC-1043 (TAC) in vivo.

Methods: SCC VII tumors transplanted into the food pads of male $\mathrm{C} 3 \mathrm{H}$ mice were used. TAC or CDDP $(2 \mathrm{mg} / \mathrm{Kg}, 4 \mathrm{mg} / \mathrm{Kg})$ were given by tail vein injection, and tumors were heated at $40^{\circ} \mathrm{C}$ or $42^{\circ} \mathrm{C}$. Anti-tumor effects were evaluated by a growth delay method. The concentrations in the blood and tumors were investigated by measuring the platinum concentrations with an atomic absorption spectrophotometer.

Results: After heating at $42^{\circ} \mathrm{C}$, antitumor effects of TAC were significantly better than those obtained by using CDDP. No increase of the effects was observed after heating at $40^{\circ} \mathrm{C}$. The concentration in blood was significantly high and the concentration in tumors after heating at $42^{\circ} \mathrm{C}$ or $40^{\circ} \mathrm{C}$ increased with heating time, but the concentration in tumors without heating did not show these changes.

Conclusions: TAC-1043 proved to be very effective when combined with local hyperthermia at $42^{\circ} \mathrm{C}$.

\section{SPACE 1809}

The prognostic value of ploidy in soft tissue sarcoma H. Pape (Presenter), C. Kölzer, Ch. Pöttgen, R. Wurm, G. Schmitt; Düsseldorf

Only few reports are available in the literature regarding the ploidy value in soft tissue sarcoma. In our retrospective analyse we evaluated the prognostic relevance of DNA content in sarcoma cells relating to tumour volume and histological grading. Since 1978 paraffin embedded material of 80 patients has been collected. The histories of these patients have been correlated to the ploidy level in the tumour cells. Overall and disease free survival probability were calculated according to DNA content, tumour volume and histological grading as reference parameters. Ploidy destination was performed by automated image cytometry (LEITAS). In 56 patients DNA measurements have been completed. The characteristics of all 80 patients were: Sex: $44 \mathrm{~F} ; 36$ $M$, Age: range 1-90 years; mean 51 years. As demonstrated in Table 1 and 2 aneuploidy was mainly seen in bulky and undifferentiated tumours reflecting a poor prognosis.
Table 1

\begin{tabular}{ccc}
\hline Histologic grading & $\begin{array}{c}\text { No. of Patients DNA } \\
\text { measured/all cases }\end{array}$ & $\begin{array}{c}\text { Ploidy } \\
\text { aneuploid/euploid }\end{array}$ \\
\hline $\mathrm{G}_{1}$ & $15 / 19$ & $4 / 11$ \\
$\mathrm{G}_{2}$ & $13 / 17$ & $6 / 7$ \\
$\mathrm{G}_{3}$ & $27 / 43$ & $23 / 4$ \\
$\mathrm{G}_{4}$ & $1 / 1$ & $1 / 0$ \\
Total & $56 / 80$ & $34 / 22$ \\
\hline
\end{tabular}

Table 2

\begin{tabular}{ccc}
\hline Tumour volume & $\begin{array}{c}\text { No. of Patients DNA } \\
\text { measured/all cases }\end{array}$ & $\begin{array}{c}\text { Ploidy } \\
\text { aneuploid/euploid }\end{array}$ \\
\hline$T_{1}$ & $14 / 19$ & $9 / 5$ \\
$T_{2}$ & $39 / 57$ & $22 / 12$ \\
$T_{3}$ & $2 / 3$ & $1 / 1$ \\
$T_{x}$ & $1 / 1$ & $0 / 0$ \\
Total & $56 / 80$ & $37 / 18$ \\
\hline
\end{tabular}

\section{SPACE 1810}

A new technique of postoperative radiation therapy in the treatment of cancer of the lower rectum

A. Vitturini, L. Aglietti, N. Raffetto (Presenter), F.Schivo, M. Trinci; Roma

This new treatment modality of postoperative irradiation of rectal cancer, allows to increase total dose from $45-50$ Gy to $60-65 \mathrm{~Gy}$, reducing complications to small intestine, with a better control on relapses.

Treatment phases are:

I - tumor resection (Hartmann colostomy); during this phase a shaped expander, outside connected by two drainage tubes, is placed in the pelvis; the device can be filled with $1500 \mathrm{ml}$ max. of physiological salt solution, to uphold the small bowel;

II - postoperative irradiation (total dose 60-65 Gy) of pelvic portal; the small bowel is outside the irradiation field when the shaped expander is filled; the bladder is shielded and to the rectal stump a maximum dose of $50 \mathrm{~Gy}$ is delivered;

III - surgical removal of the shaped expander, assessment of treatment with biopsy and recanalization by stapler.

\section{SPACE 1811}

Combined simultaneous radiation and chemotherapy as a treatment policy in locoregional recurrent breast cancer with macroscopical residual tumor

H. Renner (Presenter), M. van Kampen; Nürnberg

AIM : Our therapeutic concept in the situation of locoregional recurrent breast cancer with macroscopical residual tumor is a combined radiation and chemotherapy to increase live quality by tumor control.

Methods: 61 patients with 70 tumor areas (chestwall or/and lymphatic nodes) were treated during the last 12 years. Median follow up period was 24 months. The radiation was controlled by the rhythm of chemotherapy: 4 courses including 14 days of CMF-chemotherapy with simultaneous radiation followed by 14 days of interruption without any therapy. The overall treatment time is about $31 / 2$ to 4 months.

Results: The overall result is a $87 \%$ complete remission rate at the end of therapy and a $65 \%$ permanent local infield tumor control rate during the whole follow-up period (median 24 months). The result is depending on radiation dose. Doses higher than $60 \mathrm{~Gy}$ increase the complete remission rate to $93 \%$ and the permanent local tumor control rate (infield) to $86 \%$. The rate of ipsilateral outfield recurrences is $6 \%$ and of contralateral tumors is $15 \%$. The tolerance of this therapy is quite good. Acute side-effects (haematological, gastroenterological, degree I-II WHO) are reversible. The acute and chronical side-effects of the skin due to radiation are not more than degree I-II (EORIC).

Conclusions: Considering the problems of the macroscopical persistent tumor the therapeutic control of locoregional tumor by combined radiation and chemotherapy is a reasonable treatment policy. 


\section{SPACE 1812}

Intraoperative radiation therapy for carcinoma of the pancreas and the extrahepatic biliary system: special references on the combination of postoperative external radiotherapy

Y. Tanaka (Presenter), T. Shimizu, N. Takeshita, T. Matsuda, A. Okamoto, I. Tabata; Tokyo

From August 1976 to June 1990, ninety-one patients with carcinoma of the pancreas and fourty-five patients with carcinoma of the extrahepatic biliary system were treated with intraoperative radiation therapy (IORT). In combination with IORT, postoperative external beam irradiation was applied to about half patients in doses ranging from 30 to $54 \mathrm{~Gy}$ (average $40 \mathrm{~Gy}$ ).

The median survival time of patients with carcinoma of the pancreas was about 12 months and those with carcinoma of the extrahepatic biliary system was 8 months. The actuarial survival of these patients was $50 \%$ at 1 year and $20 \%$ at 2 years on the former, and $37 \%$ and $24 \%$ on the latter, respectively. Patients treated by IORT in combination with postoperative irradiation survived longer than those treated by IORT alone ( 13 months versus 5 months median survival on carcinoma of the pancreas and 11 months versus 3 months on carcinoma of the extrahepatic biliary system).

The treatment modality consisting of IORT plus postoperative irradiation was found to be the most important prognostic factor. After tumor resection, if possible, IORT with a dose of about $20 \mathrm{~Gy}$ combined with postoperative doses ranging from 40 to $48 \mathrm{~Gy}$ was considered to be feasible without causing severe adverse reaction.

\section{SPACE 1813}

Functional results of a therapy with a 300 Gy ablative dose in Graves' disease

U. F. Willemsen (Presenter), C. R. Pickardt, P. Knesewitsch; Munich

1. Recurrent hyperthyroidism due to Graves' disease can make an ablative radioiodine therapy necessary accepting to induce hypothyroidism.

2. Since 198527 patients ( 22 females, 5 males, age $26-70$, mean $50 \pm 13$ years) were treated by ablative radioiodine therapy because of recurrent severe hyperthyroidism. Thyroid volume was determined by ultrasound; maximum storage and effective half-time by radioiodine test. The individually required activity $(\mathrm{MBq})$ was calculated for a desired intrathyroidal absorbed dose of $300 \mathrm{~Gy}$. Post-therapy controls were performed after 3 months and thereafter in 3-6 months intervals.

3. After 3 months hyperthyroidism is eliminated in $93 \%$. 2 patients still remained hyperthyroid without needing antithyroidal drugs. The rate of hypothyroidism is $59 \%$ after $3,76 \%$ after $7,79 \%$ after 12 and $100 \%$ after 16 months. 4 euthyroid patients did not yet have their 16 months control. There is no correlation between pre-therapeutic Thyroidea-Stimulating-Immunoglobulin-(TSI) values, the applied activity and the latency period of developing hypothyroidism. Post-therapeutic TSI-values are positive in $12 / 15$ patients. Thyroid ablation with a 300 Gy absorbed dose should be restricted to recurrent cases. The main advantage to standard-activity therapies may be a reduction of the applied activity with a reliable elimination of hyperthyroidism.

\section{SPACE 1814}

Radiotherapy in the management of meningeoma

R. Wurm (Presenter), H.Pape, B.Bannach, Th. Schnabel, G. Schmitt; Düsseldorf

In our retrospective study we evaluated the role of radiotherapy in the management of meningeoma in comparison to a review of the available literature. Between 1980-1989, 14 patients with meningeoma ( 8 female, 6 male; median age: 55; range: $25-72$ ) were treated for recurrent disease with radiotherapy at our department. Initially all patients had a subtotal resection. After recurrence 10 patients underwent one or more reoperation and 4 a biopsy only. Radiotherapy was given to 5 patients after first recurrence, to 4 after second recurrence and 5 after third recurrence. Irradiation was performed with ${ }^{60} \mathrm{Co}-\gamma$-rays or high energy $x$-rays up to $45-60 \mathrm{~Gy}$ (mean $50 \mathrm{~Gy}$ ) using $5 \times 2$ or $1,8 \mathrm{~Gy} /$ week. The relapse-free survival at 5 years of $67 \%$ was better, as opposed to $26 \%$ of patients not receiving postoperative irradiation after surgery from the literature. Furthermore, the review of the available literature indicates that the relapse-free survival at 5 years of $75 \%$ of our patients treated by radiotherapy after first recurrence and reoperation was similar with those patients receiving postoperative irradiation following attempted initial surgical resection and better compared to $11 \%$ in patients treated by surgery alone after first recurrence. In conclusion achievement of local control in meningeoma appears to require whenever possible nearly complete or complete resection. Radiotherapy seems to be indicated following initial partial resection, where minimal residual disease remains. When gross residual disease remains, radiotherapy should be taken in account followed by delayed reoperation and attempted resection.

\section{SPACE 1815}

\section{Radlotherapy of thymoma}

R. Wurm (Presenter), K. Gieseler, H. Pape, D. Skutta, G. Schmitt; Düsseldorf

We performed a retrospective study on 35 patients with histologically confirmed thymoma treated at our department between 1960 and 1990.19 were male and 16 female. Median age was 45 years, with a range from 15-68 years. 10 had a complete, 22 a partial resection and 3 patients a biopsy only. 21 received radiotherapy immediately post surgery and 14 for gross recurrence. 6 had myasthenia gravis, 2 anaemia and 1 rheumatoid arthritis. 16 were lymphocytic, 10 epithelial, 7 mixed and 2 spindle cell type thymoma. 2 were classified stage I, 3 stage II, 28 stage III and 2 stage IV. Irradiation was performed up to 30-60 Gy (mean $45 \mathrm{~Gy}$ ) at 2 or 1,8 Gy daily. 12 patients had concomitant chemotherapy. The median follow-up is 9 years. For all patients the 5-, 10- and 20 -year actuarial survival rates were $71 \%, 33 \%$ and $33 \%$ respectively. The disease-free survival rates were $17 \%, 8,5 \%$ and $8,5 \%$. The 5-, 10- and 20 -year actuarial survival rates were $69 \%, 46 \%$ and $46 \%$ and the disease-free survival rates $27 \%$ at 20 years for those who had radiotherapy after surgery. In contrast, the 5- and 10-year actuarial survival rates were $75 \%$ and $0 \%$ and the disease-free survival rates $0 \%$ for those who had radiotherapy for recurrence post surgery. The review of available literature and our results suggest that postoperative radiotherapy should be given to all cases of invasive thymoma regardless they had complete or incomplete surgical excision.

\section{SE General radiology (17) SPACE 1816}

Evaluation of acute deep venous thrombosis of the lower limb, using an automated venous occlusion plethysmograph

Håkan Ahlström (Presenter), Stefan Nilsson, Andrezej Pawlowski; Uppsala

Purpose: To ascertain whether it is possible to reduce the number of phlebographic investigations by using a plethysmograph for diagnostic screening of acute DVT.

Methods: 175 consecutive patients who were referred for routine phlebography because of clinically suspected deep vein thrombosis (DVT) were also investigated with a new, simplified, computerized strain-gauge plethysmograph (Phlebotest, Eureka AB). An occlusion plethysmograph curve was obtained from each leg simultaneously. Different numerical parameters were defined and determined from curves with both fixed and variable time of cuff inflation. The parameters were correlated with the phlebographic diagnosis. Results: The diagnostic sensitivity of plethysmography with fixed inflation time was equal to that of variable inflation time. Two parameters of the outflow part of the plethysmograph curve correlated well with the phlebographic diagnosis, which proved correct in 60 patients without DVT, including 2 false negative cases, and in 36 patients with thrombosis. In 79 patients, plethysmography alone was not sufficient to establish a diagnosis. The plethysmograph described is easy to handle and a decision sequence is suggested for use in selecting those patients, with or without thrombosis, who do not require supplementary phlebography.

\section{SPACE 1817}

Presentation of a consultation system for diagnosis and therapy of bladder cancer

V. Cavallo, F. Giovagnorio (Presenter), E. Cruciani, A. Volpe; Roma

The complexity of diagnostic and stadiation protocols for bladder cancer makes the whole procedure fully understandable only from specialists. An expert system has been built containing the uroradiological expertise needed to make the process easily applicable by non-expert personnel.

The system aims at achieving a complete TNM stadiation of the tumor; the user provides, in an interactive way, the results coming from the different diagnostic methods employed, whose sequence is suggested by the system itself; in any moment informations on the status of diagnostic procedure are available, with informations on the nature and usefulness of the methods. The system provides, finally, the exact TNM stadium of the disease with appropriate therapeutic suggestions. The system has been developed using a low-cost expert system shell; the knowledge is structured in rules according to the appropriate syntax.

The applicative experiences demonstrated a great usefulness of the system, even from a didactic point of view. The definitive validation, carried on with users' suggestions, will surely make the system more complete and interactive. 


\section{SPACE 1818}

How do new diagnostic modalities influence on older methods? Urinary tract examinations in Norway 1965-1989

Tor Erik Gudmundsen (Presenter), Bjørg Vinje, Hans Kristian Pedersen, Harald Østensen; Drammen

The purpose of the study was to evaluate how the use of computerized tomography (CT) and ultrasonography (US) have influenced on the routine examinations of the urinary tract. New methods are only beneficial if they offer more information than previously obtained, or substitute other methods. The tendency in medicine has been to take up new methods in addition to other types.

Methods and Materials: For the period from 1965 to 1989, the pattern of urinary tract examinations was registered retrospectively in six Norwegian hospitals; one university hospital, two central hospitals and three local hospitals. The registrations were performed by examining the hospitals' files and protocols by two radiologists.

Results: Directly related to the introduction of CT in the various hospitals, the number of renal angiographies declined. The introduction of US and its almost linear increase reduced both the number of CT examinations and, more dramatically, the number of intravenous urographies (IVP). There was a statistically significant inverse correlation between the two modalities.

Thus, it seems reasonable to assume, that at least in Norway, new diagnostic tools for medical imaging are substituting older modalities.

\section{SPACE 1819}

\section{AIDS-related Kaposi's sarcoma}

P. Luburich (Presenter), M. C. Ayuso, C. Bru, C.Cardenal, R. Vilana, F. Gimeno; Barcelona

Purpose: To describe the imaging findings and results of staging of Kaposi's sarcoma (KS) in AIDS patients.

Methods and Materials: Between March 1985 and January 1990, 45 male patients (age range 25-53, mean 39) with cutaneous or ganglionar biopsyproved KS and AIDS were evaluated for staging purposes, 42 were homosexual (HMS), 1 intravenous drug user (IVDU), 2 HMS and IVDU. Chest radiographs (45), thoracic CT (27), abdominal C.T (38) or abdominal US (23), barium gastrointestinal examination (25) or upper fiberoptic endoscopy (30) and colonoscopy (27), and cranial CT (21) were performed at presentation, and less frequently during follow-up. By January 1990, 30 patients had died (complete autopsies 10 cases, partial 3).

Results: Lymphadenopathy or visceral lesions were present in $29 / 45(64 \%)$ cases at presentation. Visceral KS was radiologically presented as small nodules, and occasionally as larger masses. In the lung and liver, small KS nodules and tumoral bands were usually seen in a peribronchovascular (lung) or periportal (liver) location. Common sites and time of involvement were:

$\begin{array}{cccc}\text { Staging Follow- Autopsy } & \text { Total } & \begin{array}{c}\text { Pathol. or } \\ \text { endoscop. } \\ \text { documen- } \\ \text { tation }\end{array} & \begin{array}{c}\text { Based } \\ \text { on RX } \\ \text { findings } \\ \text { alone }\end{array}\end{array}$

\begin{tabular}{lrrrrrr}
\hline Adenopathy & 23 & 1 & 2 & $26(58 \%)$ & 16 & 10 \\
Gastroint. & 12 & 2 & 4 & $18(40 \%)$ & 18 & - \\
Lung & 5 & 6 & 4 & $15(33 \%)$ & 13 & 2 \\
Liver & 4 & 1 & 1 & $6(13 \%)$ & 3 & 3 \\
Spleen & - & - & 1 & $1(2 \%)$ & 1 & - \\
\hline
\end{tabular}

\section{SPACE 1820}

\section{Reduction of skin dose by new film-screen-system and additional filtering}

W. Maier (Presenter), J. Kopp, E. Klotz; Augsburg

In 1989 we changed film-screen-systems from blue to green sensitive. One goal has been to reduce radiation dose to patient without loosing image quality. Additionally we increased total filtering of the $x$-ray tubes from $2.5 \mathrm{~mm}$ Al to $4.5 \mathrm{~mm} \mathrm{Al}$.

The new orthochomatic film-screen-system with anti-cross-over films showed a much better sharpness in the images than the old blue one.

The dose reduction due to the imaging system is presented for different kinds of examinations.

The change of filtering reduces dose to the patients skin to $60 \%$.
SPACE 1821

\section{Lymphoma in AIDS}

A. Muñoz (Presenter), C. Lopez, M. A. Gonzalez, A. Alvarez; Madrid

All patients diagnosed of AIDS associated with lymphoma in our institution were reviewed. Since 1987 there were 12 cases: 11 non Hodgkin lymphoma and one Hodgkin's disease. Patients with NHL were men and age ranging from 26 to 65 years (mean 35). Risk factors were: intravenous drug abuse 6 cases, homosexuality 4, promiscuous sexual relations 2 (one also intravenous drug abuser). Kaposi sarcoma was previously diagnosed in one patient, who was included in homosexual risk group.

Histologic diagnosis was: diffuse large cell ( 4 cases), immunoblastic ( 3 cases), Burkitt ( 3 cases) and the other one was only reported as high grade lymphoma. 6 patients were clinically diagnosed as stage IV, 2 as IE, one as I and in the rest the clinical stage was unknown (one dead and other was lost to lollow-up).

7 patients presented with B symptoms at the diagnosis time. Extranodal involvement was identified in whole series: 6 cases CNS, 6 cases liver and spleen, 5 cases bone marrow, 3 cases skin and mucoses, 2 cases pleuropulmonary and one case gastro-intestinal tract. There was nodal involvement in 2 patients.

We present conventional radiologic and $\mathrm{CT}$ imaging and we compare with literature our results.

\section{SPACE 1822}

Drug effects on CA125 antigen expression and anti-CA125 antibody binding to cancer cells

Toshiharu Nakai (Presenter), Keigo Endo, Makoto Hosono, Tsuneo Saga, Harumi Sakahara, Junji Konishi; Kyoto

Purpose: In order to regulate the expression of cancer associated antigen for the immunoscintigraphy and immunotherapy, we investigated the effect of drug administration on CA 125 antigen expression and radiolabelled monoclonal antibody binding to the cancer cells.

Methods and Materials: Human ovarian and lung cancer cell lines which express CA125 antigens were cultured with various concentrations of interferon- $\gamma$, dexamethasone, or sodium butyrate. CA125 release to the culture medium was measured and after 5 days exposure to drugs, cells were incubated with radiolabelled monoclonal anti-CA125 antibody.

Results: Interferon- $\gamma$ increased the release of $\mathrm{CA} 125$ antigen and the binding of radiolabelled anti-C.A125 antibody to PC9 lung adenocarcinoma cells, but decreased A549 lung adenocarcinoma and SHIN3 ovarian carcinoma cells. Dexamethasone completely suppressed the CA 125 antigen expression in three out of 9 cell lines examined at a physiological concentration. The number of antibody binding sites decreased without affecting affinity constants. On the contrary, sodium butyrate increased CA125 expression of the same 3 cell lines more than 2-folds.

Conclusions: Drugs remarkably regulated the CA 125 expression in some, but not all, cancer cell lines. These effects may be applied to improve the tumor uptake and biodistribution of radiolabelled monoclonal antibodies.

\section{SPACE 1823}

Patterns of contrast enhancement of tuberculous lymph nodes demonstrated by computed tomography: Study of $\mathbf{3 8}$ cases

F. Pombo (Presenter), E. Rodriguez, J. Perez-Fontan, L. Arrojo; La Corunã

Purpose: To evaluate the unenhanced and postcontrast CT characteristics of pathologic toraco-abdominal lymph nodes in 38 patients with final proved diagnosis of tuberculosis.

Methods and Materials: All patients presented with constitutional symptoms and 8 had AIDS. Unenhanced scans of chest and abdomen were done in all cases. The enhanced characteristics of the largest well delimited lymph node was evaluated in a dynamic sequence over a period of 2 minutes.

Results: On unenhanced scans the nodes were of low attenuation $(<30 \mathrm{HU})$ in 18 cases and of soft-tissue attenuation $(>35 \mathrm{HU})$ in 20 . Three postcontrast patterns of enhancement were found: A) Peripheral rim enhancement without or with low to moderate increase of attenuation at the center of the nodes (22 cases), B) homogeneous enhancement ( 8 cases) and C) non-homogeneous enhancement ( 8 cases). In 7 cases a combination of $A$ and (B-C) patterns was seen at the same nodal group. The central enhancement was usually moderate (mean $30 \mathrm{HU}$ ) but in 3 instances strong enhancement (mean $60 \mathrm{HU}$ ) was found. Obliteration of perinodal fat was evident in 13 cases most of them with $A$ pattern.

\section{SPACE 1824}

Reduction of radiation dose. Benefit quality relation

E. Rodriguez (Presenter), D. Cercos, J.M. Gomez, N. Planas, A. Belles, D. Llorca, J. Ares, J. M. Nuñez; Barcelona

Our work evaluates the radiation dose received by the patient on skin by using different reinforcement screens. 
We compared regular screens (barium-sulphate) with rare earth screens (lantanum - oxibromurum). The film, X-Ray equipment and developer were the same in both cases. A phantom was used to obtain images. A "nomograma" was used to estimate the radiation dose on skin and comprobations were done with an ionization camera. Although the "nomograma" gives us just a dose orientation, this is enough to verify the decrease of radiation received by the patient by using rare earth screens.

The average obtained with different explorations shows us that the use of rare earth screens involves a reduction about $58,9 \%$ of the absorbed radiation by the patient without significative decrease of X-Ray quality.

Due to the great volume of radiodiagnostic explorations in ambulatory patients, we must optimize the radiation dose suffered by customers, even more if we consider the check controls for chronical patients which represents a high percent of our explorations.

\section{SPACE 1825}

HIV-related lymphomas: radiological findings

G. Santamaria (Presenter), M. C. Ayuso, P. Luburich, E. Salvadó, X. Tomás, C. Nicolau; Barcelona

Purpose: To study the radiological findings in HIV-related lymphomas. Methods and Materials: 23 cases of non-Hodgkin lymphoma (NHL) and Hodgkin's disease (HD) in HIV-seropositive patients, admitted at our hospital from $9 / 89$ to $5 / 90$ were reviewed. All patients were male, the mean age was 32 years (range: $22-65$ ). 14 patients $(60.8 \%$ ) were parenteral drug abusers, 6 $(26.8 \%)$ homosexual males, and $3(13 \%)$ belonged to other risk groups. Conventional radiography, ultrasonography, and computed tomography were performed.

Results: 16 patients $(69.5 \%)$ presented NHL and 7 patients (30.5\%) HD. Characteristically, all the lymphomas were of intermediate or high-grade subtypes. 13 patients $(75 \%)$ with NHL and $7(100 \%)$ with HD had advanced disease (stage 3 or 4 ) at presentation. Extensive abdominal adenopathy (retroperitoneum, mesentery) was found in 4 patients $(25 \%)$ with $\mathrm{NHL}$ and in 4 (57.1\%) with HD. 10 patients $(62.5 \%)$ with NHL and $5(71.4 \%)$ with HD had extranodal involvement. NHL: liver (2), bone marrow (3), lung (2), nervous central system, stomach, rectum, pericardium, skin (1). HD: liver (4), bone marrow (3).

\section{SPACE 1826}

Radiologic findings of worm disease

X. Tomás (Presenter), J.A. Clavero, R. Vilana, P. Luburich, T. Pujol, J. Pomés, M. Velasco; Barcelona

1. Imported diseases, which for a long time have been an unusual diagnostic, have raisen in the last years due to migratory movements towards Europe. We would like to show the most interesting radiologic findings of worm diseases observed in our hospital.

2. We have studied in a retrospective way all the cases of worm disease which have been diagnosed in our department during the last five years, and have only chosen those which we think to be more outstanding, for instance, onchocerciasis, schistosomiasis and Katayama disease, paragonimiasis, fasciolosis, Guinea worm disease ... Many imaging strategies were performed: $\mathrm{X}$-ray films, US, CT and MR.

3. We believe it is interesting to bear in mind the importance of the radiological findings of these diseases in our daily work, because of its increase of incidence in our environment.

\section{SPACE $\underline{1827}$}

Sensitivity of medical $X$-ray screen-film-processing systems in Danish hospitals

O. Hjardemaal, H. Westergaard (Presenter); Brønshøj

The sensitivity of screen-film systems is a crucial factor in doses to patients, therefore it has been determined in 25 Danish hospitals.

The sensitivity of screen-film systems in use depends on many different factors. Besides the type of screen and film can be mentioned processing, radiation quality, cassette absorption and ageing of the screens. The nominal sensitivity quoted by the manufacturer may therefore be different from the actual sensitivity.

The sensitivity can be determined according to standards, for instance the ANSI PH 2.43-1982 or the DIN 6867 standard. However, such standards are intended for laboratory use. For field use i.e. the conditions governing in X-ray departments modified principles have to be applied.

Thus, a simplified DIN method using $90 \mathrm{kV}$ and a total filtration of $32.5 \mathrm{~mm}$ of aluminium has been used and the findings are compared with the nominal sensitivity of the systems. 